


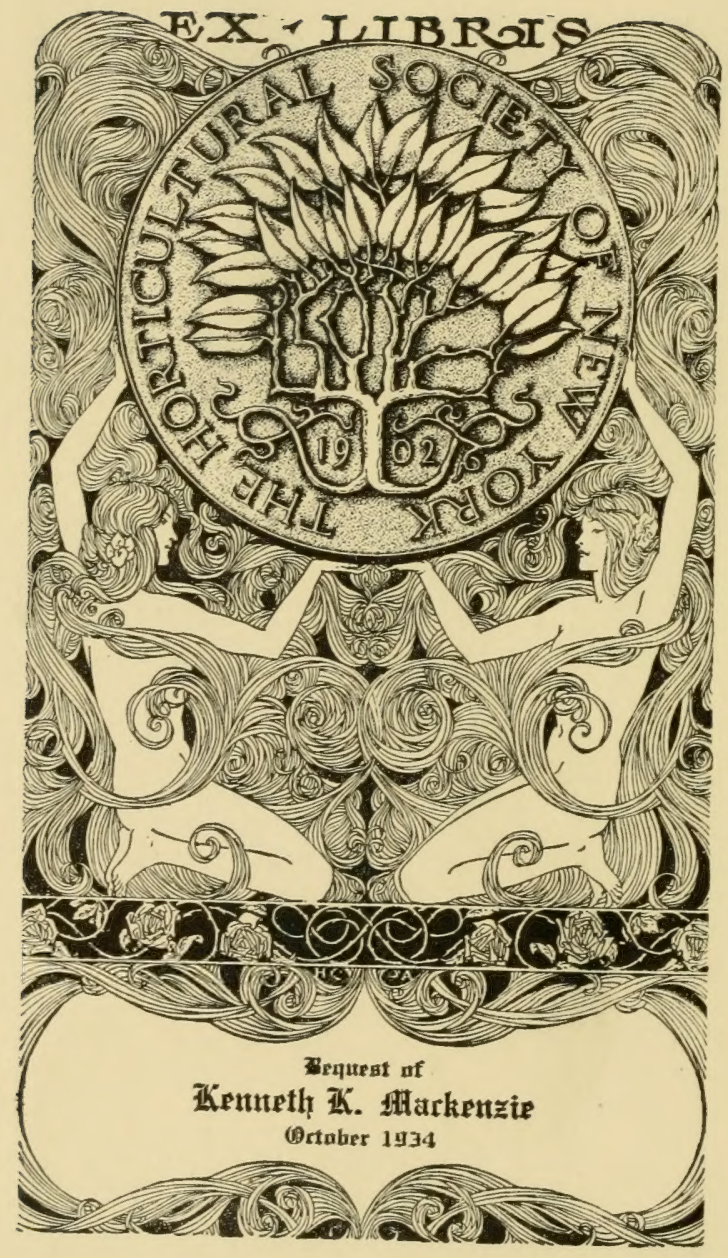

\section{THE LUESTHER T. MERTZLIBRARY}

THE NEW YORK BOTANICAL GARDEN 
. 



\title{
THE NEW YORK BOTANICAL GARDEN
}

\author{
y \\ Die
}

Gramineen Schleswig-Holsteins einschließlich des Gebiets der freien und Hansestädte Hamburg und Lübeck und des Fürstentums Lübeck.

Ton

P. Junge.

IIt zwei Kartenskizzen.

\begin{abstract}
Aus dem
Jahrbuch der Hamburgischen Wissenschaftlichen Anstalten. XXX. 1912. (3. Beiheft: Arbeiten der Botanischen Staatsinstitute.)
\end{abstract}

Ha mburg 1913.

Kommissionsverlag von Lucas Gräfe \& Sillem. 


$$
\begin{aligned}
& 34 \\
& A 1 \\
& 586 \\
& 1913
\end{aligned}
$$

$$
\begin{aligned}
& 584.9 \\
& J 95
\end{aligned}
$$

$$
10208
$$




\section{Vorrede.}

Die vorliegende Gramineenbearbeitung bildet den 3. Teil der Bearbeitung der höheren Pflanzen Schleswig-Holsteins usw. Die beiden früher erschienenen Teile sind in den Bänden XXV und XXVII dieses Jahrbuchs enthalten.

Zwei Änderungen sind gegenüber den früheren Ausführungen getroffen worden. Die Verbreitung der Gräser über die Pflanzenvereine ist fortgelassen worden. Als Südgrenze des Gebiets ist auf eine kurze Strecke die Süderelbe angenommen worden.

Wie früher, so wurde auch bei dieser Arbeit von einer Reihe wissenschaftlicher Institute mir freundliche Unterstützung gewährt, und zwar voll:

1. dem Städtischen Museum in Altona, enthaltend die Herbarien von N. Hinrichsen und J. J. Meyer, zur Verfügung gestellt von Herrn Professor Dr. Lehmann;

2. dem Botanischen Museum in Hamburg, enthaltend die Herbarien resp. Pflanzen von Bertram, Brick, Buek, Erichsen, Fischer, Griewank, L. Hansen, Jaap, A. Junge, Kirsten, Klatt, Kohlmeyer, Laban, Lienau, Möhrcker, Nolte, J. A. Schmidt, J. Schmidt, Sickmann, C. T. Timm, Zimpel und Zincke, zur Verfügung gestellt durch die Herren Professor Dr. E. Zacharias, Professor Dr. Voigt und Professor Dr. Fitting;

3. der Biologischen Anstalt auf Helgoland, enthaltend die Herbarien resp. Pflanzen von Brody, Gätke, Knuth, Kuckuck usw., zur Verfügung gestellt von Herrn Professor Dr. Kuckuck;

4. dem Botanischen Institut der Universität Kiel, enthaltend die Herbarien resp. Pflanzen von Bargum, Engelken, Haecker, Hansen, Henniges, Hennings, Hübener, Jörgensen, Kjaerbölling, von Müller, Nolte, Prahl, Reinke, Sonder, Thun usw., zur Verfügung gestellt von Herrn Geh. Regierungsrat Professor Dr. Reinke; 
5. dem Botanischen Institut der Universität Kopenhagen, enthaltend die Herbarien resp. Pflanzen von Borst, Fröhlich, Gelert, Hansen, Hornemann, Jensen, Lange, Liebmann, Mörck, Nolte, Petit, Poulsen, Rafn, Schiötz, Schumacher, W. Sonder usw., zur Verfügung gestellt von den Herren Professor Dr. Ostenfeld und Professor Dr. Warming;

6. dem Naturhistorischen Museum in Lübeck, enthaltend die Herbarien resp. Pflanzen von Brehmer, Deecke, Friedrich, Griewank, Häcker, Kohlmeyer, Lenz, Ranke, Sonder usw., zur Verfügung gestellt von Herrn Professor Dr. Lenz †.

Literatur überließen die Bibliothek des Botanischen Vereins der Provinz Brandenburg in Berlin, die Bibliotheken des Botanischen Museums, des Botanischen Vereins und der Patriotischen Gesellschaft in Hamburg, die Stadtbibliothek in Hamburg, die Schleswig-Holsteinische Landesbibliothek und die Bibliothek des Vereins für Natur- und Landeskunde in Kiel, die Bibliotheken des Botanischen Instituts in Kopenhagen und des Naturhistorischen Museums in Lübeck.

Durch Begutachtung kritischer resp. fremder Gräser förderten die Herren Geh. Regierungsrat Professor Dr. P. Ascherson in Berlin, Hofrat Professor Dr. E. Hackel in Attersee in Oberösterreich und Dr. R. Pilger in Berlin meine Untersuchungen.

Durch Überlassung und Nachweis von Literatur und Herbarmaterial und durch Auskünfte verschiedener Art unterstützten mich die Herren Seminarlehrer L. Benick in Lübeck, Lehrer M. Beyle, O. Borchmann und wissenschaftlicher Assistent Dr. Brunner in Hamburg, Mittelschullehrer A. und W. Christiansen in Kiel, Professor Dr. Friedrich in Lübeck, Lehrer W. Hansen, wissenschaftlicher Assistent Dr. W. Heering, Lehrer C. Kausch und A. Mohr in Hamburg, Oberstabsarzt Dr. P. Prahl † in Lübeck, Seminarlehrer G. R. Pieper in Hamburg, technischer Direktor H. Röper, Rektor F. Schädel und Gymnasiallehrer J. Schmidt in Hamburg, sowie stud. Franz Thorn und Fritz Thorn in Lübeck.

Allen genannten Herren, Instituten und Vereinen sei auch an dieser Stelle herzlich gedankt.

Hamburg, im März 1913.

P. Junge. 
18. Familie.

\section{Gramina.}

Jussieu Gen. plant. 28 (1789).

\section{Allgemeiner Teil.}

\section{A. Besiedlungsgruppen.}

Die Zahl der im Gebiete überhaupt beobachteten Gräserarten beträgt 197. Sie sind im speziellen Teile in drei Gruppen gegliedert worden:

1. Spontane Arten: solche, welche ausschließlich oder vorzugsweise in natürlichen Pflanzenvereinen auftreten und sich in diesen dauernd erhalten. Thre Zahl beträgt 80 , bezeichnet „sp.“.

2. Quasispontane Arten: solche, welche nur oder vorwiegend in künstlichen Pflanzenvereinen auftreten und mit dem Aufhören der Einwirkung des Menschen wie diese Formationen aus unserer Gegend verschwinden würden, die sich aber in den Vereinen der genannten Art dauernd erhalten. Thre Zahl beträgt 16, bezeichnet „qsp.".

3. Adventive Arten: solche, welche in der Regel in künstlichen, seltener in natürlichen Pflanzenvereinen auftreten und hier nur vorübergehend, oft nur durch eine Vegetationsperiode, vertreten sind. Sie sind bezeichnet „adv.".

Eine sichere Trennung ist zwischen den Gruppen nicht möglich. Am schwierigsten ist die Trennung der spontanen (einheimischen) von den quasispontanen (eingebürgerten) Arten. Der Grund liegt einesteils darin, daß eine Art in bestimmten Teilen des Gebiets einheimisch, in anderen aber eingebürgert oder auch nur verschleppt (adventiv) vorhanden sein kann, andernteils darin, daß Arten aus künstlichen in natürliche Formationen übergehen und so in ihrer Existenz von den vom Menschen geschaffenen Pflanzenvereinen unabhängig werden können.

Alopecurus pratensis, Bromus inermis, B. commutatus und B. tectorum sind im Elbgebiet in natürlichen Formationen vertreten, also spontan; sonst aber finden sie sich im Gebiete eingebürgert (A. pratensis und viel- 
leicht auch $B$. inermis [wenn nicht adventiv]) oder verschleppt ( $B$. tectorum und B. commutatus). Brachypodium pinnatum ist einheimisch im Land Oldenburg und vielleicht bei Lauenburg; außerdem ist es zuweilen adventiv beobachtet worden.

Von den drei erstgenannten Arten hat Alopecurus pratensis die weiteste Verbreitung erlangt; die Aussaat als Futtergras erklärt das. Während dieses Gras im Elbgebiet in natürlicher Formation für das Gebiet nachgewiesen ist, ist ein anderes Gras überall in unserem Florengebiet erst aus künstlichen Formationen in die natürlichen, ihm zusagenden Genossenschaften eingedrungen, nämlich Lotium multiflorum. Es ist hier heute durchweg nicht selten und tritt in einer Weise auf, welche ein späteres Verschwinden aus den betreffenden Pflanzenvereinen ausgeschlossen erscheinen läßt. Das Gras ist daher unter die spontanen Glieder unserer Gräserflora gestellt worden.

Aus der zweiten Reihe, der der eingebürgerten Gräser, erscheinen Panicum lineare, P. crus galli, Trisetum pratense, Poa Chaixii und Festuca dertonensis ( $F$. sciuroides) zuweilen in natürlichen Formationen. Doch ist dies Auftreten ein nur ausnahmsweises und spielen z. T. die betreffenden Formationen eine so untergeordnete Rolle, daß es nicht ratsam erscheint, auf Grund des Erscheinens der genannten Arten in ihnen die Arten als spontan zu bezeichnen. Es handelt sich um Pflanzengenossenschaften, die, wie sandiges Grasland, lichte Gebüsche usw., zwar zu den natürlichen zählen, aber hier und dort durch die menschliche Kultur nicht beeinträchtigt, sondern sogar (allerdings ungewollt) begünstigt werden. Die übrigen Arten der Gruppe (Anthoxanthum aristatum, Panicum viride, P. glaucum, Alopecurus myosuroides, Agrostis spica venti, Bromus erectus, B. secalinus, B. arvensis, Hordeum murinum, Lolium temulentum und L. remotum) sind stärker an die Kulturformationen gebunden als die erstgenannten Spezies.

Die Trennung der adventiven Arten ist einfacher, wenn auch die eingebürgerten Arten adventiv auftreten können, mit Ausnahme der Poa Chaixii, welche, außer in Parks und Grasgärten, nur im Walde (Buchenwald; vielleicht ursprünglich mit ihm in unsere Gegend gekommen?) beobachtet worden ist. Auch manche spontane Gräser zeigen sich zuweilen als Adventivpflanzen. $\mathrm{Zu}$ erwähnen sind in dieser Hinsicht besonders einige salzliebende Arten: Festuca distans, Hordeum secalinum und $H$. maritimum. Für ihre Eingliederung war das Auftreten im natürlichen Pflanzenverein entscheidend (so auch z. B. bei Bromus commutatus).

Als die Gletscher am Schlusse der Eiszeit von Schleswig-Holstein zurückwichen, wanderten von Südwesten, Süden und Osten unsere heimischen Gräser ein, doch nicht gleichzeitig, sondern in Gruppen oder einzeln in 
verschiedenen Zeiten. Die Wanderung ist für manche von ihnen noch heute nicht abgeschlossen.

Als die Gletscher zuriekwichen, lebte im kalten Wasser der Ostsee eine nordische Wuschel, Yoldiu rotirn; nach ihr heift die Ostsee jener Zeit Iolder-See, die Zeitperiode Joldiu-Zeit. sie lag nach allerdings unsicheren Schätzmgen etwa vom 15̃.--10. Jahrtausend v. Chr. Das Klima der Küstengebiete an der Voldir-See war ein ähnliches wie hente im nördlichsten Teile Rublands. In jener Zeit gedieh an zahliejohen Orten des norddentschen Flachlandes die boreal-alpine Diyns ortoputuln, deren Reste in Moorbildungen verschiedentlich beobachtet wuden. I)ir P'eriode erhielt damach die Bezeichnung der Dryns-Zeit. Diese lag trilweise ibereinstimmend mit der Yoldia-reit.

An die Zeit des häufigsten Auftretens der Dryas schloß sich eine solche grober Häufigkeit der Birke, die Birkenzeit, welche vielleicht mit dem letzten 'Teile der Toldia-Zeit zusammenfiel.

Eine Hebung: Südskandinaviens (und der cimbrischen Halbinsel?) schnitt die Yoldia-See von der Nordsee (ganz oder fast ganz) ab. Der starke Zustrom süben Wassers von den immer mehr abtauenden ciletschern führte zur Aussïbung der Ostsee. Ioldia artice verschwand; ihe stelle nahm Ancylus lacustris ein. Das Meer war die Ancylus-See, die Periode ihrer Existenz die Aucylus-Zeit. Ihr entsprachen in der Florenentwicklumg die Kiefern- und die Eichenzeit und wahrscheinlich die Zeit der Einwanderung pontischer Florenelemente.

Eine S'nkung der Landgebiete von sü̈dskandinavien bis Norddentschland brachte aufs neue eine Terbindung zwischen Tord- und Ostsee. cin Findringen salzigen Wassers in die Ostsee und infolgedessen u. a. die Finwandermo der Litorina litoren in die Ostsee. Es entstand das LitninuMeer. Die Periode seines Bestehens, die Litorinn-Zeit, beginnt mit der. Litorinu-Senkung. Nit ihrem Beginn stimmte der Beginn der Buchenzeit meln oder weniger überein. Die Zeit der Senkung war vielleicht das ó. und 4. Jahrtausend r. Chr.

An die Litorima-Zeit schloß sich die Mya-Zeit, die etwa mit dem letzten (1.) Jahrtausend v. Chr. begamn und noch fortdauert.

In der Dryas-Zeit bewohnten unsere Gegend zahlreiche arktischalpine Pflanzen, darmuter, obgleich in Resten nicht bokannt. Wolll anch Gräser. Von ihnen werden von den noch hente vorhandenen Arten in erster Linie solche in Betracht lommen. Welehe in den fiebirgen die Kältegrade bedentender Höhen ertragen und (oder) hente arktische tiebiete

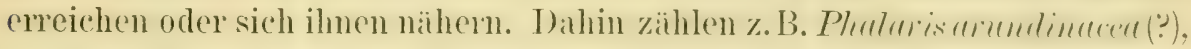
Anthoxanthum odoratum, Hierochloe odorata, Mitium effusum (?), Nardus

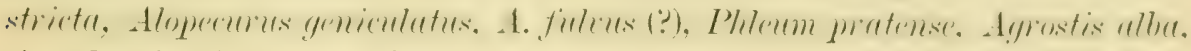
A. vulgaris, A. canina, Calamagrostis lanceolata (??), C. villosa, C. neglecta, 
C.epigeios(?), Comenaria(?), Holcuslanatus, Acena pubescens (?), Aera flexusas. A. crexpitose, A. Wibctiana (vgl. unten), Sieglingia decumbens, Arumdo phragmites, Motinia coerulea, Poa anmua (?), P. nemoralis, P. trivialis (?), P. matensis, Briza media (?). Cutabrosa aquatiea, Glyceria fluitans, G. plicata,

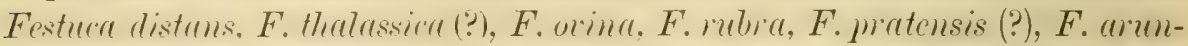
dinacea(:), C'ynosurns sristatus, Bromushordeaceus, Triticum repens, T.junceum, Horderm seralimum, H. arenarium und Lolium perenne. Die mit? bezeichneten Arten lassen nach ihren Terbreitungsareal und ihren Pflanzenvereinen die Möglichkeit einer Einwanderung in einer der nächsten Perioden offen.

In der Birken-, Kiefern- oder Eichenzeit (wahrscheinlich in einer der beiden letzten, also in der Anylus-Zeit) dürften die folgenden Arten in unser Gebiet eingewandert sein: Alopernms pratensis, Phleum arenarium (?), Holcus mollis, Avena clatior, A. pratensis, Aera caryophyllea, A. praecox,

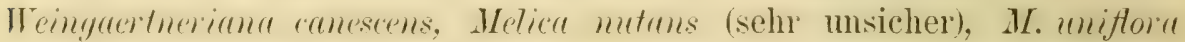
(oder Buchenzeit?), Kosteria glauea. Dactylis glomerata, Poa palustris, P. compressa, Glyceria aquatica (oder früher), Bromus inermis, B. steritis, B. tectorum, B. racemosus (oder später?) und Brachypodium pinnatum.

Eine recht geschlossene Gruppe brachte dam die Buchenzeit in den Buchenbegleitern unter den Gräsern. In diese Gruppe gehören Festurn

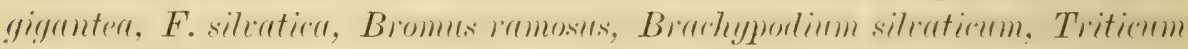
canimum und Hordeum europaeum, vielleicht auch Glyceria nemoralis.

Als Einwanderer der Mya-Zeit könnte Lotum multiflorum gelten.

Soweit die Gruppengliederung; jetzt einige Bemerkungen zu einigen Arten und Artengruppen.

Volı unseren spontanen Gräsern sind nem Arten obligate oder fakulta-

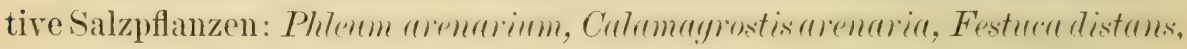
F. thalassica, Triticum junceum, Hordeum secalinum, H. maritimum, H. arenarium und Lepturus incurrotus. Ton ihnen werden an der kalten ToldirSee nur die kälteertragenden Arten vorhanden gewesen sein, die Arten also, welche heute weit nordwärts vorkommen. Es sind das Calcumagrost is arenaria, Festuca distans, Triticum junceum und Hordeum arenarium. An der Aurylus-See werden sie entweder ganz eingegangen sein oder sich wenigstens stark zurückgedrängt gesehen haben. Vielleicht haben sie die Zeit der Ungunst an den Salzstellen der Randgebiete der Saxonischen Scholle gegen den russisch-baltischen schild überdanert. Als dann die Litmina-Zeit eintrat, drang Salzwasser in die Ostsee und damit waren die Bedingungen für eine ernente Ausbreitung dieser Salzgräser gegeben.

Phleum arenarium, Festuca thalassica, Hordeum secalinum und Lepturus immmotus gehen nord- und ostwärts nicht allzu weit über unser Gebiet hinaus. Das dentet auf späte Einwanderung hin. Mit Ausnahme von Hondrum sernlimm treten sie nicht an binnenländischen Salzstellen auf 
(Phlenm revnurum anf salzfreien Simdfächen im Rhein- und Rhonegebiet). I)as läfot vermuten, dal.j die Arten auch an den Salzstellen des (iebiets der Anrylu-Zeit gefehlt haben. Ist das der Fall, so kömen sie erst in der Litorina-Zeit eingewandert sein. An der Vordsee mögen sie bereits früher vorhanden gewesen sein. Phlenm rrmminm reicht an der Ostsee bis Hiddensee, Lrpturns imrmentus bis Mönchgut auf Rügen. Hordenm sereflinum bis zur Odermündung und Festure thalussing bis Kolberg. Nordwärts gehen sie bis ins suidliche Skandinavien.

Hordeum maritimum findet sich nur an der Nordseeküste auf beschränktem Gebiete ron Dithmarschen bis Dagebüll. Der Bezirk ist ron dem zusammenhängenden Areal der Art durch eine breite Lnterbrechung im Elbmïndungsgebiet getremnt. Die Tremumg ist vielleicht auf die Litorinr-Senkung zurückzuführen, welche die rerbindenden Standorte unter der Meeresoberfäche verschwinden ließ.

An den Leptwr-Standorten der schleswig-holsteinischen Ostseeküste ist auffällig, daf sie sämtlich auberhalb der Föhrden liegen; am dichtesten und mit dem reichsten Bestande des Grases auf Fehmarn und in Land Oldenburg. Der Grund für die Verbreitung und Häufigkeit liegt im Salzgehalt des Wassers, das auch für das Terschwinden einiger Salzoräser nich Osten entscheidend ist. Die vier salzbedürftigeren Arten sind eben dieser Änderung in Salzgehalt des Wassers wegen fast ganz auf die westliche Ostsee (im Sinne Reinkes) beschränkt.

Zu den interessantesten Arten der Toldir-Zeit gehört Arra Wibmirnar. Thre nächsten Verwandten sind A. lavigatu (besonders in Nordemropa: von Island und Irland bis Lappland), A. bottried (im nördlichen Ostseegebiet) und A. litoralis (in den Westalpen an Fluß- und Seeufern). A. botnica und A. litoratis unterscheiden sich von A. Wibeliana leicht durch die lange Granne, welche die Hüllspelzen beträchtlich (bis $3 \mathrm{~mm}$ ) überragt. A. Tareigata steht der. A. Wibeliana darin nahe, das ihre Deckspelze eine kurze, die Hüllspelze nicht überragende trame trägt. Sie unterscheidet sich ron letzterer Art durch geringere Gröbe, gefaltete Bläter, glatte Rispenäste und dumkle Alnchen. Die Unterschiede sind wenig charakteristisch. Die Ähulichkeit beider Arten legt die Termutmmg. gemeinschaftlicher Abstammung nahe. Sollten beide sich aber nicht ron einer Stammart herleiten, so bestände die Möglichkeit, dals die eine auf die andere zurüchginge, und zwar A. Wibeliunr in ihrer Entstehumg anf die rerbreitetere A. lumigratu. Daß letztere Art früher bei uns rorkam, ist sehr wohl möglich, da sie heute in einem groben Teile ilues Areals mit Diyas ortopetalu zusammen auftritt. Irgendwie sichere Schlüsse sind allerdings nicht möglich.

Da Aren Tibeliann im Gebiete des Ebbe- und Flutwechsels auftritt und ausschlieblich an durch diesen Wechsel getrotfenen Örtichkeiten 
gedeiht, ist nicht ansgeschlossen, dafo die Entwicklung der Art im Anschluß an die besonderen Bedingungen dieser Orte und als Folge der dort vorhandenen Verhältnisse geschah.

Die Kiefer hat das Areal, das sie in der Kiefernzeit immehatte, bis auf das Gebiet zwischen Lübeck und Geesthacht vertoren. Hier finden sich anch die natürlichen Standorte von Koeterin glanca, Bromus inermis und $B$. tectorum. Die Arten greifen nicht wesentlich über die Kiefergrenze im Südosten hinaus. Sie sind zwar nicht im Auftreten an die Kiefer gebunden, lieben aber Verhältnisse, die auch der Kiefer zusigen. Das führt auf die Termutung, daf ihre Einwanderung in der Kiefernzeit erfolgte.

Mit der Eiche kamen vermutlich Calamagrostis arundinacea und Brachypodium pimnatum. Beide lieben lichten Wald, wie ihn Eichen oder Eichen-Buchen-Aischbestände geben. Conlamagrostis armudinaren kommt anch im lichten Eichenkrattwald vor. Ihre weit rerstreuten Standorte im Gebiet des schleswig-holsteinischen Mittelrückens, dem früher vielfach große Bestände lichten Eichen-oder Mischwaldes zukamen, denten auf ehemaliges häufigeres Vorhandensein.

Auffällig gleiche Verbreitung haben im südlichen Teile des besprochenen

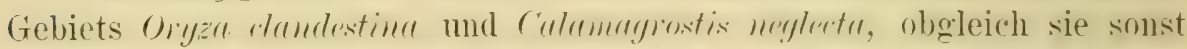
durchans verschiedenen Verbreitung:gruppen angehören. (Jiyz» rl. ist ein südlicher Typus später Einwanderungszeit, dessen Nordgrenze durch sichleswigHolstein geht (England-Eider-dänische Inseh-Südschweden-Rubjand). Culumagrostis n. aber ist ein nordischer Typus früher Einwanderungweit. dessen süd-(Südwest-)grenze mit der südgrenze des behandelten Gebiets sich eine Strecke lang deckt, z. T. ins Gebiet fällt (rgl. Skizze). ('ulmumgrostis villosa in Angeln ist als Relikt anzusehen.

Es ist bereits erwähnt worden, daß die MLya-Zeit Lolium multiflorum gebracht hat. Diese Zeit ist von den früheren dadurch unterschieden, daß in ihr der Mensch eine über der natürichen Entwicklung und L'mbildung der Pflanzenvereine stehende, verändernde. und zwar sowohl rernichtende wie neuschaffende Tätigkeit entwickelte. Seine Tätigkeit brachte direkt oder (durch Sichaffung nener Ansiedelungsmöglichkeiten) indirekt die (iruppe der quasiopontanen Arten in unsere Gegend. In den übrigen Zeiten fehlte zwar nicht der Mensch im Gebiet, aber seine Einwirkmg. anf die Pflanzenwelt war wenig umfassend, ja geringtügig und damit mehr oder weniger bedeutungslos.

Soweit über die Zeit der Einwanderung:

Sind Arten durch misere Gegend weiter nordwärts gewandert, haben ilne Terbreitungsgrenze weiter vorgeschoben gegen Norden coder anch ()sten oder Westen), so kam unser Gebiet zom zentralen Areal des Auftretens der betreffenden Arten gezählt werden. Das ist bei der weitaus 
größten Mehrzahl der spontanen Arten der Fall, bei to derselben ron sol. Die fünf übrigen spezies finden im fiebiet eine Grenze des natürlichen

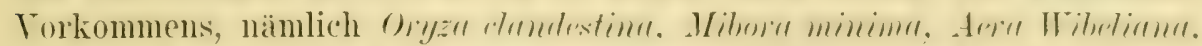

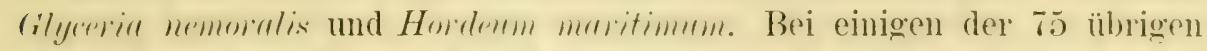
Arten liegt eine Grenze des Verbreitumgsareals nicht weit über uns'r Florengebiet hinans: die betreffenden spezies reichen bis Dänemark und sïdskandinavien resi). bis Ostdentschland. Dahin zählen (die trenzen sind z. T. schon erwähnt) z. B. Phleum arenarium, Calamagrostis amudinacea, Aera caryophyllea, A. praceox, A. discolor, Koeleria glauca, Festuca thalassica, F. sitvatica, Brome tectorum, Hordeum exropaenm und Lepturus incurvatus.

Aera Wibeliana kommt bei uns im Nordwesten Dentschlands endemisch vor.

Mibora minima und Hordeum maritimum sind Angehörige des atlantischen Artenkreises, die in Schleswig-Holstein Nord- und zusleich Westgrenze haben. Mibora minima reicht von der Pyrenäenhalbinsel durch Frankreich und Belgien bis siudengland. in die Viederlande und bis ins Rheingebiet. Einzelne standorte sind weit vorgeschoben. Dahin gehören anch die Vorkommen im Gebiet. die vielfach. aber meines Erarchtens ohne genügende Begriundung. als nicht spontan betrachtet worden sind

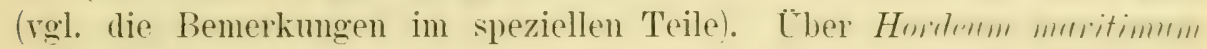
ist bereits gesprochen worden.

Fïr (Jyza rlumestinn geht die Nordgrenze durch Schleswig-Holstein. Das tras reicht ron süden bis südengland, Schleswig-Holstein. Inseln Dänemarks, sïdschweden und Mittelrubland (bis zum bo. dirad). Tremn die Art für unsere fregend als Wanderpflanze bezeichnet worden ist. so ist. das nicht richtig. Sie wandert heute anscheinend nicht mehr.

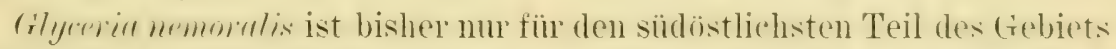
(bei Ratzeburg und Reinfeld) nachgewiesen. Sie hat hier ihr westlichstestorkommen. das gegenüber den früher als Grenzfundorten geltenden standorten bei stettin. Landsberg a. W. Meseritz. Liegnitz. Hirschbero. Leitmeritz. Eisenbug usw. weit westwäts roloeschoben ist. Walnseheinlich werden sich zwischen Ratzeburg und stettin weitere Fundorte nalchweisen lassen.

Die beiden letzterwähnten Gräser gehören zur Gruppe der Arten, welche ron osten resp. südosten her ant die cimbrische Halbinsel gelangt. sind, deren südliche Hälfte schleswig-Holstein aber nicht ganz durchwandert haben. Dahin gehören z. B. von spontanen Arten Alopecurus fulver, Melica mutans, Pon compressa, Bromus inermis, B. tertorum und

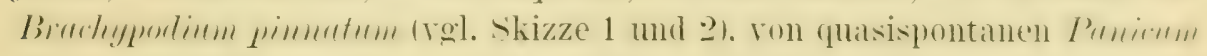
lineare und $P$. glauam. Anthoxanthum aristatum, Paniam arus galli, P. viride, Festuen dertonensis und Hordeum muimum sind in Schleswig; besonders im nördlichen Teile, weit seltener als im Süden. 
Sehr bedeutend ist in der Familie der Gramineen die Anzahl der eingeschlepyten Arten. mit 101 grober als die der spontanen und quasispontanen zusammengenommen. Janche ron ihnen treten bereits in Jittel- und süddeutschland spontan auf. so z. B. Phlemmit Bumlemeri.

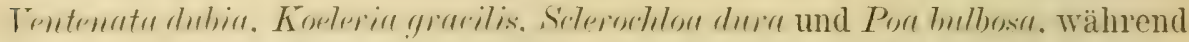
andere dort wenigstens quasispontan vorhanden sind. wie Panirum sanguruale.

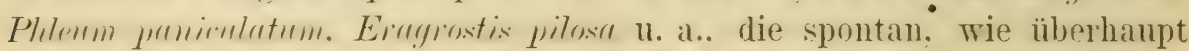
die meisten der verschleppten Gräser, dem Mittelmeergebiet angehören. Die in Betracht hommenden Pflanzen finden sich z. T. nicht im ganzen Mittelmeergebiet. sondern nur im Westen desselben (dann oft zugleich in Westemplat) oder nur in seinem ostlichen Teile (dam oft zugleich im Pannonischen Bezirk oder weit nach Asien hinein): einige der Arten sind fast rein pamonisch. Hierher zählen z. B. Phalnris amoriensis. P\%. minor Ph. paradoxa, Andropogon halepense, Tragus racemosus, Panicum italicum, P. verticillatum, Nitium vemale, Stupa tortilis, Alopecurus utriculatus,

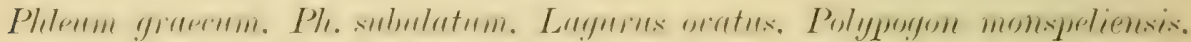
Agrostis verticillata, A. nebulosa, A. intermpta, Trisetum paniceum, Aera

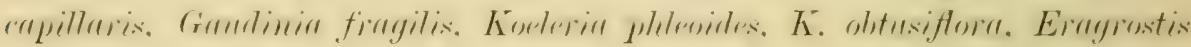
megastachya, E. minor, Schismus calycims, S' 'arabicus, Briza maxima,

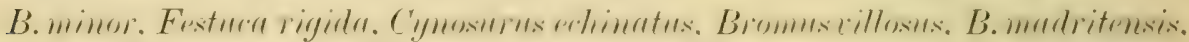
B. squarrosus, B. scoparius, B. macrostachys, Brachypodium distachyon, Triticum cristatum, T. villosum, T. ovatum, T. triunciale, T. cylindricum, Lotium rigidum und Psiluras aristatus.

Westasien lieferte die Getreidearten häufiger Kultur: Hafer-, Weizenund Gerstenarten sowie Rogeren, auch z. B. Punimm milinrmm. Mrvlin

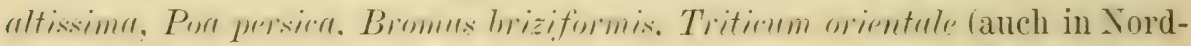

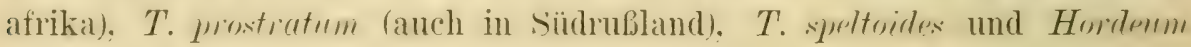
spontaneum.

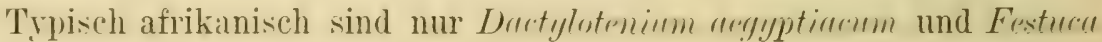
angusta.

Aus Nordamerika stammen Panicum capillare, Cenchrus tribuloides,

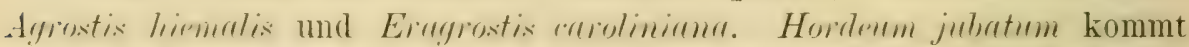
auch in sïdamerika ror, aus dem verschiedene Gräser eingeschleppt wurden 1\%. T. mit Wolle, besonders aus Argentinien): Paspalus inemosus. Chloris babata, C. radiata, C. truncata, Elensine indica, E. tristachya,

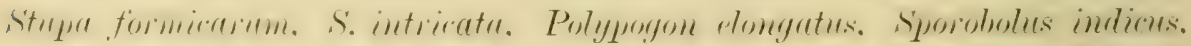
Bromus uniotioides und Hordeum compressum. Ton ihnen sind Chloris

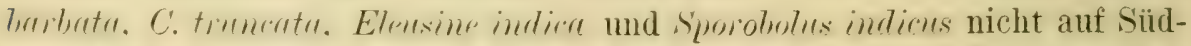
anerika beschränkt, sondern in den Tropen der ganzen Erde heimisch. Agrostis retrofracta hat ihre Heimat in Australien. 


\section{B. Verbreitungsgruppen.}

Drei Hauptzonen sind im Gebiet zu tremen:

a. Das Hügelgebiet: Es bildet die östliche Zone. Seine Hauptentwicklung hat es längs der Ostsee in einem breiten Giurtel, von dem ans einzelne, sehr ähnliche Bezirke bis zur Alster und Elbe sowie ins mittlere Holstein und in den Kreis Hadersleben vorspringen. Diese letzteren lassen sich als Unterzonen trennen:

$\alpha$. Elbgebiet: von der Delvenaumündung bei Lauenburg bis unterhalb Hamburgs (a. $\boldsymbol{\alpha}_{\text {. }}$.

B. Südliches Übergangsgebiet: von Ratzeburg-OldesloeSegeberg bis zur Elbe und Alster. in gewisser Weise dem Heidegebiet genähert und von Partien desselben (bis Trittan ostwärts) durchsetzt (a. $\beta$.).

$\gamma$. Nördliches Übergangsgebiet: ron Sommerstedt-Woyens bis Gramm-Rödding (a. $\gamma$.).

$\delta$. Salzgebiete: sie fïgen sich als besonderer Bezirk an, und zwar teils an der Ostsee (a. $\delta .1$.), teils binnenlands (a. $\delta .2$.).

b. Das Heidegebiet: Es bildet die mittlere Zone und begleitet das Hügelgebiet auf der Westseite in mehr oder minder breitem Streifen. Nur in Schleswig erreicht es die Nordsee; sonst ist es ron ihr durch die Narsch getrennt. Zu ihm zählen zwei Unterbezirke:

$a$. Geestgebiete der Nordseeinseln (b. $c_{0}$ ).

$\beta$. Salzgebiete: sie liegen sümtlich an der Nordsee iteils auf dem Festlande, teils auf den Inseln) (b. $\beta$.).

c. Das Marschengebiet: Es wird durch die Bodenbeschaffenheit charakterisiert, die ihre Eigenart der Entstehungsweise verdankt. Sie ist in der Hauptsache Seemarsch. Aus dieser Bezeichnung ergibt sich als Gegensatz der eine Unterbezirk, außer dem noch ein zweiter durch die Anwesenheit von Natriumchlorid unterschieden werden muß:

๘. Flußmarsch: an Elbe und Eider (c. $\alpha_{\text {o. }}$.

$\beta$. Salzgebiete: an der Nordsee (c. $\beta$.).

Von den 80 spontanen Arten des Gebiets sind vertreten:

1. Oryza clandestina: a (südlicher Teil), b (südlicher Teil), c. $\propto$.

2. Phularis armulimuren: a. b, c, überall ziemlich gleichmäßig: fehlt b. a.

3. Anthoxanthum odoratum: a, b, c.

4. Hierochloe odorata: a, c verbreiteter, b selten.

5. Mitum effusum: a häufig, b zerstrent; b. a unsicher. 
6. Vardus stricta: a, b, c; c seltener.

i. Nitora minima: b (südlicher Teil).

\&. Alopecurus pratensis: a, b, c; ursprünglich nur a. $a$ und c. $a$.

9. Alopecunes geniculatus: a, b, c (selten an Salzorten).

10. A7opecums fulcus: $\mathrm{a}, \mathrm{b}, \mathrm{c}$; c seltener.

11. Phleum pratense: a, b, c.

12. Phleum arenarium: a. $\delta$ (nur im südlichen Gebiete), b. $\beta$.

13. Agrostis alba: a, b, c (auch Salzorte).

14. Agrostis vulgaris: a, b, c (auch Salzorte).

15. Agrostis cenine: a, b, c; c selten.

16. Calamagrostis lanceolata: a, b, c; b. a selten.

17. Calamagrastis villosa: a (nur im nördlichen Gebiete).

15. Calamagrostis neglecta: a, b; c?; (nur im siidlichen Gebiete).

19. Calamagrostis amendinacea: a. $\beta$ (zerstreut), b (selten).

20. Calamagrost is epigeios: a, b, c; c selten.

21. Calamagrostis arenaria: a, b, c; besonders a. $\delta$ und b. $\beta$ (Dünen).

2.2. Holcus lanatus: a, b, c.

23. Holcus mollis: a, b, c; c selten.

24. Acena elatior: $a, b, c$.

25. Avena pubescens: a, b, c; c selten.

26. Avena pratensis: a, b.

27. Aera caryoplyyllea: a, b, c; c seltener.

28. Aera praecox: a, b, c; c seltener.

29. Aera flemosa: a, b, c; c seltener.

30. Aera setacea: b (im Süden selten).

31. Aera caespitosa: a, b, c.

3:2. dera Wibetiana: c. «.

33. Teingartneria canescens: a, b, c; c seltener.

34. Sieglingia decumbens: a, b, c.

35. Arundo phragmites: a, b, c (auch Salzorte).

36. ITolinia coemulea: a, b, c; c seltener.

:3-. Melica mutans: a, b; b selten.

Bis. Melica uniftora: a, b; b seltener.

3. Koeleric glauca: a (fast ausschließlich a. $\boldsymbol{a}_{\text {. }}$ ), b selten (nur im Norden).

41. Durtylis glomerata: a, b, c.

41. Por anmea: a, b, c (auch an Salzorten).

42. Por nemoralis: a, b, c; c reniger verbreitet.

43. Poa palustris: a, b, c; c selten.

44. Pon compressa: a; b, c selten.

45. Poa trivialis: a, b, c.

46. Poa pratensis: a, b, c. 
47. Briza media: a, b, c.

48. Catabrosa aquatica: a, b, c; c weniger oft.

49. Glyceria fluitans: a, b, c.

50. Glyceria plicata: a; b und c wenig.

51. Glyceria nemoralis: a (selten und nur im südöstlichsten Gebiet).

52. Glyceria aquatica: a, b, c.

53. Festuca distans: a $(\delta, 1$ und $\delta$. 2), b (sehr wenig b. $\beta$ ), c. $\beta$.

54. Festuca thalassica: a (nur a. $\delta, 1)$, b. $\beta$, с. $\beta$.

55. Festuca ovina: a, b, c; c weniger oft.

56. Festuca rubra: a, b, c (auch Salzorte).

57. Festuca pratensis: a, b, c.

58. Festuca arundinacea: a, b, c (auch an Salzorten).

59. Festuca gigantea: a, seltener b, c.

60. Festuca silvatica: a (doch a. $\beta$ und a. $\gamma$ seltener); b sehr vereinzelt.

61. Cynosurus cristatus: a, b, c.

62. Bromus ramosus: a (doch a. $\beta$ und a. $\gamma$ selten); b sehr wenig.

63. Bromms inermis: a. " (sonst nur infolge Aussaat oder Verschlepjung).

6t. Bromus sterilis: a, b, c; c selten.

65. Bromms tretormm: a. ce (und rielleicht bis Travemünde durch a. $\beta$ und a) heimisch.

66. Bromus racemosus: a, b, c.

67. Bromus hordeaceus: a, b, c (auch Salzorte).

68. Bromus commutatus: a. a und c. $\alpha$ (sonst nur adventiv).

69. Brachypodium pinnatum: a (nur im Südosten).

70. Brachypodium sitvaticum: a, b; (b selten).

71. Triticum caninum: a, doch a. $\beta$ und a. $\gamma$ seltener.

7๖. Triticum repens: a, b, c (auch Salzorte).

73. Triticum junceum: a. $\delta .1$ und b. $\beta$, c. $\beta$.

74. Hordeum secalinum: a. $\delta$. 1, b. $\beta$, c. $\beta$, weniger a. $\iota$, b. $\alpha, c, \alpha$.

75). Hordeum maritimum: c (ob nur c, $\beta$ ?).

76. Hordeum enropaeum: a (a. $\beta$ und a. $\gamma$ selten).

77. Hordeum arenarium: a. $\delta .1$, b. $\beta$, c. $\beta$; (sonst nur verschleppt).

78. Lolium pereme: a, b, c (ob auch Salzorte?).

79. Lolium multiflorum: a, b, c.

80. Lepturus incurvatus: a. $\delta .1$, c. $\beta$; (b. $\beta$ ?:).

Bei der Feststellung ist jedesmal nur spontanes Torkommen beriicksichtigt worden. Ton den 8() spontanen Gräsern sind 56 Arten in allen drei Gebieten vertreten, allerdings nicht in allen gleichhaufig: zwölf weitere Spezies sind wenigstens in zwei dieser Zonen vorhanden (z. T. in der dritten nur unsicher). 
Es erscheinen von 80 Arten:

\begin{tabular}{c|c|c}
\hline in drei Zonen & in zwei Zonen & in einer Zone \\
\hline $56=70 \%$ & $13=16,25 \%$ & $11=13,75 \%$ \\
\hline 1. & 2. & 3. \\
\hline
\end{tabular}

Aus Spalte 3:

\begin{tabular}{c|c|c}
\hline $\begin{array}{c}\text { nur in } \\
\text { Hügelgebiet }\end{array}$ & $\begin{array}{c}\text { nur im } \\
\text { Heidegebiet }\end{array}$ & $\begin{array}{c}\text { nur im } \\
\text { Marschengebiet }\end{array}$ \\
\hline $7=8,75 \%$ & $2=2,5 \%$ & $2=2,5 \%$ \\
\hline
\end{tabular}

Es sind vertreten:

1. nur im Hügelgebiet: Calamagrostis villosa, Glyceria nemoralis, Brachypodium pinnatum, Bromus inermis, Bromus tectorum, Triticum caninum und Hordeum europaeum.

2. nur im Heidegebiet: Hibora minima, Aera discolor.

3. nur im Narschengebiet: Aera Wibeliana, Hordeum maritimum.

Von den zwölf Arten, die in je zwei Zonen als Angehörige vorhanden sind, finden sich zehn in Zone a und b, eine in Zone a und c und eine in Zone b und c. Als Gesamtzahlen ergeben sich:

1. für Zone a: $56+11+7=74$ Arten $=92,5 \%$ der spontanen Gräser;

2. für Zone b: $56+1+2=59$ Arten $=73,75 \%$ der spontanen Gräser;

3. für Zone c: $56+2+2=b 0$ Arten $=75 \%$ der spontanen Gräser.

Es zeigt sich hier wie bei den Halbgräsern und Farnpflanzen das Hügelgebiet als das artenreichste. Während aber bei jenen beiden Gruppen die Marsch hinter dem Heidegebiet an Zahl der Arten zurückstand, ist das bei den Gräsern nicht der Fall.

Der bei weitem gräserreichste Teil des besprochenen Gebiets ist der Südosten von Hamburg bis Lübeck und Travemïnde; hier fehlen nur Calamagrostis villosa und Hordeum maritimum. 


\section{Spezieller Teil.}

\section{A. Bestimmungstabelle der Gattungen.}

1. Ährchen getrenntgeschlechtlich; männliche Ährehen in endständiger Rispe, weibliche Ährehen in blattachselständigen Kolben...Zea.

- Ährchen sämtlich oder größtenteils mit Zwitterblüten........ ?

2. Ährchen ungestielt in einfacher Ähre oder sehr kurz gestielt in ahrenartiger Traube ....................... 3

- Ährchen nicht in einfacher Ähre oder Traube............ 12

3. Deckspelze mit rürkenständiger, geknieter, im unteren Teile gedrehter Granne.

Gaudinia.

- Deckspelze grannenlos oder mit gerader Granne.

4. Ährchen sämtlich oder größtenteils mit einer Hüllspelze oder ohne Hüllspelzen

- Ährchen sämtlich mit zwei Hüllspelzen

5. Ährchen ohne Hüllspelzen oder mit einer kleinen, undentlichen Hüllspelze; Stempel nur mit einer Narbe

Nardus.

- Seitenährchen mit einer Hüllspelze, selten mit zwei (dann in der Regel sehr ungleichen) Hüllspelzen; Endährchen mit zwei Hüllspelzen; Stempel mit zwei Narben .................

6. Alle Seitenährchen mit einer Hüllspelze, sitzend ..........

- Seitenährchen wenigstens zum Teile mit zwei Hüllspelzen, wenigstens die unteren mit kurzem, zuweilen der Achse angewachsenem Stiel ... . . . . . . . . . . . . . . . . . . . Festulolium

7. Ährchen 1-2-blütig, lineal; Hüllspelze viel kleiner als die untere Deckspelze ........................ Psilurus.

- Ährchen mehrblütig, nicht lineal; Hüllspelze so lang wie die unterste Deckspelze oder (oft beträchtlich) lïnger....... Lolium.

8. Ährchen sitzend .......................... 9

- Ährchen kurz gestielt ...................... 10

9. Ährchen der Achse dicht angedrückt, in Achsenaushöhlungen stehend; niedriges (iras salzhaltiger Orte des Strandes. . Lepturus.

- Ährchen der Achse nicht angedrückt........... Triticum.

10. Ährehen unter $2 \mathrm{~mm}$ lang; Deckspelze gramnenlos...... Mibora.

- Ährehen über 2 mm lang; Deckspelzen gramnenlos und begrannt 11 
11. Ährlıen an der Arhse in zwei einander gegenuberstehenden Reihen

Brachypodium.

- Ährchen an einer Seite der Achse, mehr oder weniger einseitswendig

Festuca z. T.

12. Ährhen sitzend in Ähen oder sehr kurz gestielt in ährenartigen Trauben, welche fingerig oder rispig gestellt sind ......... 13

- Ährhen länger oder kürzer gestielt in Rispen, die locker oder ährenartig zusammengezogen sind .

13. Ahrohen rerschiedenartig: je ein mittleres. weibliches und zwei

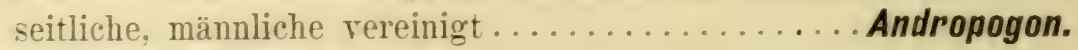

- Ahrehen gleichartig........................... 14

14. Ährchen mit einer Zwitterblüte ................ 15

- Ährchen mit zwei oder mehr Zwitterblüten............. 18

15). Ährohen nit drei Hüllsuelzen. einer kurzen. oft undentlichen unteren und zwei gleichen oder etwas ungleichen oberen.

Panicum z. T.

- Ihrchen mit zwei Hüllspelzen ................. 16

16. Thren fingerig gestellt. ....................... 17

- Ähren seitlirh der Achse in ungleicher Höhe. risjig... Paspalus.

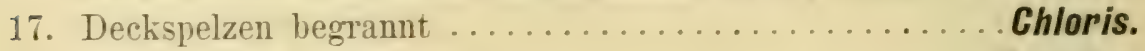

— Deckspelzen unbegrannt................. Cynodon.

18. Thren fingerig gestellt. . . . . . . . . . . . . . . . . . 19

- Ahren traubig oder rispig gestellt. ................. 20

19. Ähre mit einem Endährchen............... Eleusine.

- Ähre nhme Endährohen. ihre Achse in eine vpitze ausgezogen.

Dactylotenium.

20. Änchen zweiblütig: Aste mehr oder weniger einseitig gestellt.

Beckmannia.

- Ährohen vier-bis mehrblitig: Äste spiralig gestellt. . Diplachne.

21. İnchen simtlich mit einer Zwitterblïte (zuweilen z. T. olne Zwitterblüts und damn unfruchtbar oder mit männlichen Blüten); zuweilen neben del Zwitterblüte noch ein bis zwei männliche Blïten............................... 22

- Ährchen nit zwei oder mehr Zwitterblitten (selten Ährchen

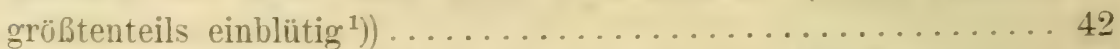

2.2. Alrrhen hüllspelzfnlus oder mit drei oder vier Hüllspelzen.... 23

- Ährchen mit zwei Hüllspelzen ................... 27

1) Ist unter den Ahrchen einer Rispe eine Anzahl zweiblütig, so ist mit Wahrscheinlirhk-it anzun hmen. dafs das Gras normalerweise zweiblütige Ährchen besitzt. denn tin. Herabstzuner dor Blütenzahl in mehr oder weniger zahlreichen ährehen scheint riel rerhreiteter zu ofin als der exerenteilige fall einer ausnahmsweisen Vermehrung der Blütenzahl in normal einblütigen Ährchen. 
23. Hüllspelzen fehlend, verkümmert ............. Oryza.

- Hüllspelzen rorhanden.

24. Ährehen mit drei Hüllspelzen, am Girunde von Borsten umgeben, ohne die Borsten abfallend .............Panicum 7. 'T'. Ährchen mit vier Hüllspelzen, am Grunde borstenlos ........ 25

25. Die beiden oberen Hüllspelzen begrannt ............ 26 Die beiden oberen Hüllspelzen grannenlos.........Phalaris.

26. Untere Hüllspelzen (1. und 2.) gleich oder fast gleich, wenig lünger als die beiden oberen; die beiden oberen Hüllspelzen (oder zuweilen nur eine von ihnen) mit je einer männlichen Blüte.

Hierochloe.

-- Erste Hüllspelze etwa halb so lang wie die zweite; dritte und vierte Hüllspelze ohne männliche Blüte ........Anthoxanthum.

27. Ährchen neben der einen Zwitterblüte mit ein bis zwei mämlichen Blüten. .

Ährchen ohne mämnliche Blüten ................... 31

28. Ährchenachse unbehaart . . . . . . . . . . . . . . . . 29

Ährchenachse behaart .......................... 30

29. Männliche Blüten unter der Zwitterblüte...... vgl. Hierochloe. Nännliche Blüte über der Zwitterblïte.......... vgl. Melica.

30. Untere Blüte stärker begrannt als die obere ...... vgl. Avena. Obere Blüte stärker begrannt als die untere...... vgl. Holcus.

31. Ährchenachse mit Haaren von wenigstens ein Viertel der Deckspelzenlänge; Haare in dichtem Kranze........ Calamagrostis.

- Ährchenachse kahl oder kurz behaart; Haare nicht in dichtem Kranze . . . . . . . . . . . . . . . . . . . . 32

32. Ährchen am Grunde von stacheligen Borsten umgeben

Cenchrus.

- Ährchen ohne Borstenhïlle..................... 3:

33. Hüllspelzen (eine oder beide) behaart oder stachelig ........ 3t

- Hüllspelzen kihl. ......................... 35

34. Beide Hüllspelzen behaart; alle Ährehen fruchtbar ... Lagurus.

- Untere Hüllspelze glatt, klein oder zuweilen ganz tohlend; obere Hüllspelze stachelig; Achsenzweige mit zwei oder mehr fruchtbaren und einem verkümmerten, unfruchtbaren Ährchen... Tragus.

35. Ährchen nicht seitlich zusammengedrückt ........... 36

- Ährchen (zuweilen nur schwach) seitlich zusammengedrückt ....3

36. Ährehen höchstens $3 \mathrm{~mm}$ lang; Deckspelze mbegrannt... Milium.

- Ährchen viel länger; Deckspelze mit langer, das Ährchen weit

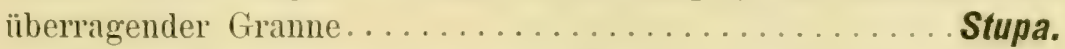

37. Hüllspelzen gleich oder fast gleich; Rispe dicht .......... 38

- Hüllspelzen deutlich ungleich; Rispe locker ............. 40 
38. Beide Hüllspelzen unbegrannt

- Hüllspelzen begrannt.

Polypogon.

39. Hüllspelzen nicht verwachsen, bei der Fruchtreife sitzen bleibend; Deckspelzen unbegrannt.

Phleum.

- Hiillspelzen im unteren Teile oft rerwachsen; Ährchen bei der Fruchtreife als Ganzes abfallend: Dechspelzen begrannt... Alopecurus. Vol. Aera praecox!

40. Deckspelzen am Rürken algerundet, mit drei starken Nerren.

Vgl. Catabrosa.

- Deckspelzen mit deutlich herrortretendem Mittelnerr, ohne oder mit schwachen Seitennerven................... 4

41. Blatthäutchen durch einen Haarkianz ersetat; Deckispelze und Vorspelze etwa gleich lang; Frucht nicht von Deck- und Vorspelze umschlossen, frei

Sporobolus.

- Blatthäutchen vorhanden; Torspelze kürzer als die Deckspelze oder fehlend; Frucht von der Deck-(und Vor-)kpelze eingeschlossen

Agrostis.

42. Deckspelzen grammenlos, zuweilen mit fein anserezogener spitze oder gezähnt-stachelspitzig ................... 43

- Deckipelzen einer Blüte oder aller Blüten im Ihrehen brgramnt 59

43. Ährehen teils fruchtbar, teils unfruchtbar: die fruchtbaren von den unfruchtbaren umgeben.......................

- Ährchen gleichartig ........................ 44

44. ت̈hrehenachse mit sehr verlängerten, die Deckspelze an Länge ïbertreffenden Haaren . . . . . . . . . . . . . . Arundo.

- Ährchenachse kahl oder kurz behaart............... 45

45. Ährchenachse wenigstens unter der untersten Blüte kumz behairt 46

- Ährchenachse kahl ........................ 48

46. Hüllspelzen länger als das Ährchen, dasselbe einschliefend . . . . 47

- Hüllspelzen kürzer als das Ärrehen; Stengel nur nahe dem firunde mit Knoten . . . . . . . . . . . . . . . . . . Molinia.

47. Ährchen länglich-eiförmig; Hüllspelzen drei- his viernervig.

Sieglingia.

_-_ Ährehen lanzettlich; Hüllspelzen sieben- bis mehrnervig.

Vgl. Avena.

48. Deckspelzen am oberen Ende zweizähnig (zweispaltig) ....... 49

- Deckspelze spitz oder stumpf, ungeteilt.............. 50

4?. Hüllspelzen länger als säntliche Decksjelzen

Schismus.

- Hüllspelzen so lang oder kürzer als die unterste Deckspelze.

Vol. Bromus.

50. Hüllspelzen so lang wie die Ährchen oder wenig kürzer...... 51

- Hüllspelzen beträchtlich kürzer als die Ährehen .......... 52 
o1. Rispe dicht; Ährehen deutlich zusammengedrückt; alle Blüten zwitterig

Koeleria.

- Rispe locker; Ährchen wenig zusammengedrückt; ein bis zwei untere Blüten zwitterig; die ïbrigen (oberen) männlich.

Melica.

52. Deckspelzen an Rücken (besonders unterwärts) abgerundet. ilı Mittelnerv nicht dentlich hervortretend .............. 53

- Deckspelzen gekiclt, mit deutlich hervortretendem Mittelnerr... כ่

53. Ährchen (ein- bis) zweiblütig, nur etwa $2(-2,5) \mathrm{mm}$ lang.

Catabrosa.

Ährchen drei- oder mehrbliitig, über $3 \mathrm{~mm}$ lang .......... 54

54. Ährchen nicht oder wenig länger als breit, mit stumpfen, am Grunde herztormigen Deckspelzen; Frucht ungetureht ... Briza.

- Ährchen beträchtlich länger als breit; Deckspelzen nicht herzförmig: Frucht geturcht .

5ว. Beide Hüllspelzen einnervig; Frucht frei.

- Obere Hüllspelze dreinervig; Frucht mit Deck- und Torspelze ausfallend

Festuca.

อ̆ 6 . Hüll- und Deckspelzen stumpf; Deckspelzen im unteren Teile knorpelig

Sclerochloa.

- Hüllspelzen spitz; Deckspelzen stmupt oder spitz. nicht knorpelig 57

57. Ährchen in Gruppen dicht gedrängt. geknäuelt....... Dactylis. Alurchen entfernter, nicht geknäuelt................ 58

58. Rispenäste spiralig um die Achse gestellt: Ihrchenachse zäh. bei der Fruchtreife bleibend. nicht zerfallend

Eragrostis.

- Rispenäste nach einel Seite gestellt: ̈̈hrehenachse bei der Fruchtreife zerfallend ........................

Vgl. Diplachne!

59. Granne endständig, zuweilen zwischen zwei Seitenspitzen eingesenkt.

-. Gramne rückenständig

6(). Hüllspelzen etwa von Ährchenlänge

Koeleria.

Hüllspelzen beträchtlich kürzer als das Ährehen

61. Ährehen in Gruppen dicht gedrängt. geknäuelt. nit am Kiel neistens gewimperten Deckspelzen................ Dactylis.

-_- Ährchen nicht geknäuelt; Decksjelzen, wenn gekielt, am Kiel nicht gewimpert . . . . . . . . . . . . . . . . . (i:

62. Rispe mit dreiseitiger Achse: Deckivelze allmählich in die cramme ausgezogen

Festuca.

- Rispe mit vierseitiger Achse; Deckspelze zwischen zwei kuzen, seitlichen Zähnen oder eben unterhalb des Einschnitts begrannt. 
6i3. irame in der Mitte gerliedert. am oberen Ende kenligr rerdicht. Weingaertneria.

- Granne einfach oder gekniet, nicht rerdickt........... 64

154. Ähroh zweiblütig: obere Deckopelze begrannt. untere unbegrannt rider selten legrannt. dann aber mit schwächerer Grame als die obere Deckspelze .

- Ährchen zwei- bis mahnlütor: untere Deckspelze mit stärkerer

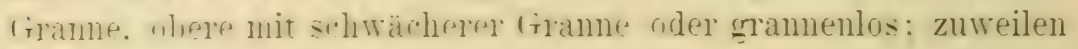
alle Deckspelzen begrannt ................... 66

65. Ährehen +-1$) \mathrm{mm}$ langr. bei der Fruchtreife als fanzes abfallend; Granne bis 5 mm $\operatorname{lang} . \ldots \ldots \ldots \ldots \ldots \ldots$ Holcus. Ahrchen etwa $1 \mathrm{~cm}$ lang; Frucht mit der Dechspelze aus den

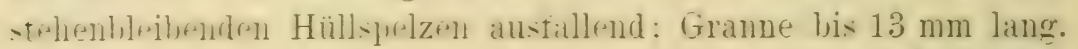

Ventenata.

Tgl. Aera praecox mit etwa $2 \mathrm{~mm}$ langen Ährehen!

66. Fruchtknoten behaart; Ährchen in der Regel ïber $1 \mathrm{~cm}$ lang.

Avena.

Fruchthnoten kahl: Alnchen klein ..............6 67

fiт. B̈hrehen arei- und melnblütier. selten z. T. zweiblütigr . Trisetum.

- Ihwhen in der Pecel zwriblitig selten einzehe lis viele Ährchen einblütig [Sect. Caryophiyllea] oder noch seltener Ahrchen z. T. dreiblütig [Sect. Deschampsia]) .......................

\title{
B. Gattungen und Arten.
}

\author{
1. Gattung.
}

\section{Oryza.}

L. Gen. plant. ed. 5. 155 (1754).

1. (sp. 1). Oryza clandestina А. Braun Bot. Ter. Brandenb. II. 195 (1861).

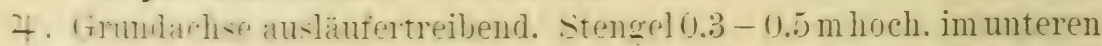
Teile meistens stark verästrlt. autrecht oder ans niederliegendem (srunde aufsteicend. meistens glatt. mit kurzharigen Fusten. Blattscheiden grob rürchwäts raulı. Blattlärohe in der Regel $0.4-11.9 \mathrm{~cm}$ breit. am Rande stark rauh. Blatthäutchen sehr kurz. grestutzt. Rispe meistens cob bei 
uns immer?! ganz oder zum gröbten Teile nicht aus der soheide herrortretend. mit rauhen. am Grunde oft geschlägelten Ästen. 肖rchen $4-5 \mathrm{~mm}$ lang. fast $2 \mathrm{~mm}$ breit, einblütig. Hüllspelzen fehlschlagend. Dechispelze gekielt. dreinervig. an hiel mol Rändern stark gewimpert; Trrspelze ähnlich. etwas schmäler. Staubbläter 3. Narben 2. Frucht frei. seitlich zusammengedrücht, am Grunde verschmälert. etwa :' mm lang. Blüte August bis Oktober.

An Lferrändern und auf fenchten Wiesen an gröberen Bächen. an Flüssen. Teichen und seen sowie aut dem Boden austrocknender oder abgelassener Teiche hesonder's Fischteicher wder aut Bagrererde im südïstlichen (tebiet bis an die Eider. doch nenerdings seltener und ansoheinend vielfach verschwunden.

L a u n burg: bei der Donnerschleuse bei Mölln (Nolte 1823!), an

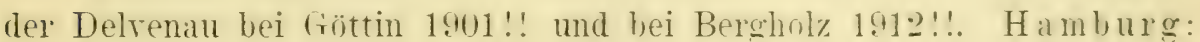
an der Bille (Flügge nach Flora Danica t. 174t [1 203$]$, bei Beroedort (Nolte 1820!). Billwärder (sickmam narh Enmeratio. 1).6 [1836]) und hei der ..Blauen Brücke" (Hübener nach Fln'a ron Hamburg 492 [1-47]). C. Timm 1852 nach Naturw. Ter. Hamburg N. F. IV. 6s [1-90)]. TI. Timm D. Bot. Ges. IX. 126 [1842], Zimpel 1891!) Hamm Nolte. Hb. Kiel!! mehrfach (Hübener usw. !). Hornerdeich (Zimpel 1s91 !). Allemïhe (sonder') aut Baggererde im Hammerbrook 1.T. A. Schmidt 1875!) am hl. (irasbrook (C. Timm. J. A. schmidt 1879!) und am Kïhlbrand 1.T. A. Schmidt 1868!. Laban 1869!). Pinneberg: am Diebsteich in Altona (C. Timm), an der Elbe unterhalb Blankenese isteetz nach sickmann. am Lier der Aue in Ellerbek (C. Timm). Stormarn: bei Trittau am Stenzerteich Tolte 1821 .. T. A. Schmidt 1866:. Laban 15.7!) und an einem Teiche in der Hahnheide (C. Timm). bei Oejendort und bei schleems i.T. A. schmidt 1865 !). an der Alster bei Poppenbiuttel (R. Timm 1904)!.. Preetz (Weber

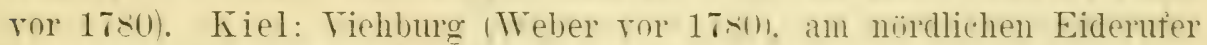
gegenüber Hasenkrug (A. Christiansen 1912!). Rendsburg: an der Eider bei Nübbel (Hansen 18+1!. Hinrichsen 1853!). Ahlheck 1Hansen 1845!). Breiholz (Nolte 1833!) und Prinzenmonr (Nolte 1823!). Dith marschen: Tresselburen (J. J. Meyer 1821!, Nolte 1823!, 1825!).

Die erste Erwähnung aus dem Gebiet findet unsere Alt als Ehrluritic rombstimn bei ITeber (Primitiale Flurae Holsaticae 6t [17s0] ron Tiehburg und Preetz.

Im Kieler Herbar liegen zwei Exemplare olne standorts- und Sammlerangabe ron 17s1! und 1790 !: rielleicht stammen sie ron Weber. Lngenau ist in diesem Herbar eine Standortsangabe von Nolte: "An der Stechnitz" 1404!; rielleicht stammen die Pfanzen ron der Domnersehleuse. Lngenügend testgestellt und durch Exemplare nicht belegt ist die Angabe aus den Besenhorster Wiesen bei Geesthacht) Bertram nach Knuth Fl. 
Schlesw.-Holst. usw. 752 [1887], Laban nach Fl. v. Holst. 222 [1866]). Mit der Angabe rom Köhlbrand ist der Standort Kuhwärder (A. Junge in Verhandl. Ver. Naturw. Unterh. 96 [1890], Laban in Fl. v. Hamburg usw. 3. Aufl. 17t [1877]) identisch. Konuths Fundort: zwischen Steinbek und Oejendorf (Fl. v. Schlesw.-Holst. usw. 752 [1887]) mach Bertram ist das gleiche Vorkommen, das J. A. Schmidt als Oejendorf nent; Bertram stand mit Schmidt in Terbindung. Kunth gibt (a. a. O.) noch Grönwohld als Standort, Hansen als Finder an; auch hier geht die Notiz auf einen ron einem andern Sammler nachgewiesenen Ort (eine der beiden Trittauer Fundstellen) zurück. Unrichtig ist der Termerk: häufig auf Wiesen bei Schönberg (Cohrt nach Kunth a. a. O.), entstellt in gleichem Werke der Ortsmame .. ITesterbuhr", der richtig Wesselburen heiben mub. Die Unformung geht auf einen sichreibfehler im Provinzialherbar zurück, wo Noltes Bezeichnumg .. Wesslingluren“ in .. Westerburen" rerwandelt ist.

Alle von mir gesehenen Exemplare des (rebiets gehören zu, f. im lusul Wiesbaner in Baenitz Herb. Europ. XXXI. 3062 (1877). welche ron Batnitz (I)entsche Bot. Monatssehr. XT. 21 [1897]) charakterisiert wird: Halme in dichten Rasen, bis 0,50 m hoch; Blätter dunkelgrün; Blüten- und Fruchtrispe dauernd von den Blattscheiden eingeschlossen und dicht zusammengedrängt. Im Gegensatze dazu stehen beif. $f$ prten: (Wiesbaner a. a. 0.3063) die Halme rereinzelt, werden bis $2 \mathrm{~m}$ hoch, tragen gelblich-grüne Blätter und eine hervortretende Rispe. Mit dem verschiedenen Verhalten der Rispe hängt vielleicht das Auftreten kleistogamer und chasmogamer Blüten zusammen, erstere mit $0,7 \mathrm{~mm}$, letztere mit $1.6 \mathrm{~mm}$ langen Antheren (rgl. Hackel in Österr. Bot. Zeitschr. LVI. 82 [1906]).

\section{Formen.}

Nicht beobachtet.

\section{Gattung.}

\section{Phalaris.}

L. Gen. plant. ed. 5. 29 (1754) z. T.

1. Pflanze 24; Rispe groß; lappig; Hüllspelzen mit ungeflügeltem Kiel.

$P$. arundinacea.

- Pflanze $\odot$; Rispe dicht, eiförmig bis zylindrisch; Hüllspelzen mit geflügeltem Kiel

2. Obere Hüllspelzen deutlich entwickelt (etwa $3 \mathrm{~mm}$ lang); Kiel der Hüllspelzen nicht gezähnt .............................. canariensis. 
- Beide obere Hüllspelzen oder doch eine von ihnen fehlend oder winzig klein...

3. Ährchen sämtlich gleichartig, fruchtbar ..................... minor.

- Ahrchen ungleichartig, ein fruchtbares jederseits von drei unfruchtbaren begleitet, mit ihnen zusammen abfallend ................ paradoxa.

2. (adv. 1). Phalaris canariensis L. Supee. plant. ed. 1. 54 (1753).

$\odot$. Stengel in der Regel aufrecht, oft vom Grunde verzweigt, (1),1-)0,3-(0,5 m hoch, glatt. Blattscheiden schwach rauh, Blattflache 0,4-0,8 cm breit, kahl. Blatthäutchen $0,3-0.4 \mathrm{~cm}$ lang, stumpf. Rispe eiförmig oder oval, $2-3,5 \mathrm{~cm}$ lang. Ährchen gedrängt, kurzgestielt, bis $8 \mathrm{~mm}$ lang, einblütig, mit vier Hüllspelzen. Lntere Hüllspelzen $6-8 \mathrm{~mm}$ lang, dreinervig, kahnförmig mit breitem Kiel, kahl. Obere Hüllspelzen gleich, etwa $3 \mathrm{~mm}$ lang; lanzettlich, kahl. Deckspelze am Rücken dicht kurzhaarig, etwa $5 \mathrm{~mm}$ lang, wenig länger als die von ihr umfaßte schmälere, kihle Vorspelze. Staubbläter 3. Frucht etwa $3 \mathrm{~mm}$ lang. bei der Reife von Deck- mol Torspelze fest mmschlossen. Blïte Juni bis Oktober.

Anf Schutt. Baggererde, (iarten- und Gemïseland in rielen Gegenden des (iebiets, besonders in der Umgegend der Städte. nicht selten, indessen anf den Nordfriesischen Inseln bisher nicht festgestellt. wohl aber beobachtet auf Helgoland (Hallier usw.)!!.

Buek nennt als erster P. canariensis für Hamburg (Hoppes Bot. Taschenb. 91 [1801]). Das älteste eingesehene Exemplar fand Bargum 1791 bei Kiel!.

\section{Formen.}

Nicht beobachtet.

\section{Mißbildungen.}

f. m. vivipara nov. f. - Ährehen in Laubsprosse auswachsend.

H a mburg: Steinwärder (J. A. Schmidt 1865!), Kl. Grasbrook (Laban 1865!). Beide Orte vielleicht identisch.

3. (adv. 2). Phalaris minor Retzius Observ. bot. III. 8 (1779/91).

$\odot$. Stengel meistens aufrecht, unterwärts oft verzweigt, $(0,2-)$ $0.4-0,8 \mathrm{~m}$ hoch, glatt. Blattscheiden schwach rauh. Blattfläche (2-) $4-6 \mathrm{~mm}$ breit, kahl. Blatthäutchen $4-5(-7) \mathrm{mm}$ lang, am Rande zerrissen, stumpt. Rispe in der Regel zylindrisch, 2-5 cm lang, 1-1.5 cm breit. Ährchen gedrängt, kurzgestielt, $5(-6) \mathrm{mm}$ lang, einblütig, mit drei Hüllspelzen. Untere Hüllspelzen etwa 5 mm lang, dreinervig, schmäler gekielt, oberwärts mit gezähneltem Hautrand, mit kurzer, aber dentlicher 
silitze. Ton den zwei oberen Hüllspelzen eine entwickelt. $1-1.5 \mathrm{~mm}$ lang. lanzettlich. die andere fehlgeschlagen. Deckspelze etwa $3 \mathrm{~mm}$ lang. die Torspelze und Frucht fest umschließend. Frucht etwa mm lang. Blüte .Tuli bis Oktober.

Auf Schutt bei Hamburg.

$\mathrm{H}$ a mburg: in Eppendort beim Eppendorterbanm (Lahan 1890!). bei Wandsbek auf dem Königslande (.T. Schmidt 1892!) und bei der Trandsbeker Dampfmühle (Zimpel 1894! usw.). Georgwwärder (Zimpel 1893!). bei der Wollkämmerei an Reiherstieg (Zimpel 1896 !. Mohr 1906!). bei der Holstenbranerei in Altona (Zimpel 1892! usw.). in einer Kiesgrube in Bahrenfeld 1901!!.

Für das Gebiet zuerst erwähnt im 2. Bericht des Bot. Vereins zu Hamburg (Deutsche Bot. Monatsschr. XI. [1893]).

\section{Formen.}

f. gracilis Parlatore Fl. Ital. I. $70(18+8)$. - $0.2-0.3 \mathrm{~m}$ hoch, feinstengelig. Rispe kurz, armblütig. Ährchen $3 \mathrm{~mm}$ lang.

Hamburg: bei der Wollkämmerei am Reiherstieg (Mohr!), bei der Wandsbeker Dampfmühle (J. Schmidt 1894!).

\section{4. (adv. 3). Phalaris paradoxa L. spee. 1)int. ed. 2. 1665 (1773).}

C. stengel aufsteigend, am Grunde stark verzweigt. $0.3-0.5 \mathrm{~m}$ hoch. oft rauh. Blattscheiden schwach rauh, die oberste deutlich aufgehlasen. Blattfläche 3-5 mm breit. rauh. Blatthäutchen 3-4 mm lang. zerschlitz.t. Rispe fast zylindrisch, nach oben und unten verschmälert. $3-4 \mathrm{~cm}$ lang. $1--1,5 \mathrm{~cm}$ breit, bei der Reife in Gruppen ron je sieben Ährchen zerfallend: daron das mittlere Ahrehen $6-8$ mm lang. fruchtbar, die seitlichen 2-4 mm lang. untruchthar. Untere Hüllspelzen des firuchtbaren Ährehens gekielt. am Kiel mit einem etwa $1 \mathrm{~mm}$ langen Zahn. mit allmählich ausgezogener Grannenspitze ron etwa $2.5 \mathrm{~mm}$ Länge. Obere Hüllspelzen tast fehlend. Deckspelze kahl, glänzend, die Torspelze und Frucht umschliefend. Frucht etwa $2 \mathrm{~mm}$ lang. Blüte Juli bis September.

Auf Schutt bei Hamburg und Kiel.

$\mathrm{Hamburg:}$ in Eppendorf beim Eppendorferbaum (Laban 1890!\%. bei der Wandsbeker Dampfmühle (Zimpel 1894! 1895!). bei der Wollkïmmerei am Reiherstieg (J. schmidt 1894! usw.), bei der Holstenbranerei in Altona (Zimpel 1892!). bei Langenfelde (Laban 1892!. Zimpel 1893!). Kiel: Neumühlen (A. Christiansen 1909!).

\section{Formen.}

Nicht beobachtet. 
5. (s). 2). Phalaris arundinacea L. sipee. plint. ed. 1. 80 (175:3).

4. Stengel 0,5-2 m hoch, aufrecht, glatt, aus langkriechender (irmudachse. Blattseheiden glatt. Blattfläche $6-12(-20)$ mm breit, oberseits schwath rauh. Blatthäutehen meistens $2-4 \mathrm{~mm}$ lang, stark zerrissen. Rispe s-20 cm lang. gelaput, in der Blüte meist ansqebreitet, sonst zusammengezogen, mit zahlreichen öhrehen an ihren zweigen. Ährehen kurz gestielt. Untere Hüllspelzen fast gleich, etwa 4 mm lang, in der Regel grün, dreinervig, gekielt: Kiel ungeflïgelt. Obere Hüllspelzen etwa $1 \mathrm{~mm}$ lang. schmal lanzettlich, zottig behaart. Deckspelze :3 mm lang. glimzend. spit\%, behant. Frucht $2 \mathrm{~mm}$ ling, schwach zusammengedrïckt. ron Deck- und Torspelze umschlossen. Blüte Juni und Juli, zuweilen August.

An Gewässern und in Sümpfen durch das Gebiet häufig, doch anf den Nordseeinsehn nur auf Fïhr zerstreut (Schiötz Videnskab. Meddelels. naturhist. Foren. Kjöbenharn 140 [1861])!!.

Für das Gebiet zuerst erwähnt von Weber (Primitiae Fl. Hols. 6 [17-0]), früher schon für das damalige Dänemark ron Oeder (Flora Danica t. $259[1766])$.

\section{Formen.}

1. Rispe locker oder dicht; Stengel astlos:

f. typica nov. f. - Rispe locker; untere Hüllspelzen grïn oder schwach violettbraun überlaufen.

Häufigste Form.

Dazu: f. coarctate Prahl Krit. Fl. Schlesw.-Holst. 245 (1890). - Rispe dicht zusammengezogen, mit sehr verkürzten Ästen. Untere Hüllspelzen dunkelviolett überlaufen.

An trockenen Orten zerstreut; zuerst am Einfelder See beobachtet.

- Rispe locker; stengel ans einigen oder allen Kinoten Äste treibend: Äste zuweilen rispentragend:

f. ramifera P. Junge Jahrb. Hamb. Wiss. Anst. XXII. 60 (1905). (Ob f. ramosa Gaudin Fl. Helv. I. 160 [1828]?).

So auf nährstoffreichem Boden zerstreut, z. B. La u enburg: Sandkrug bei Lauenburg und Gr.Pampau!!. St ormarn : Poppenbüttel!!

\section{Mißbildungen.}

f. m. picta (L. Spec. plant. ed. 1. 80 [1753]), — Blätter weißgestreift.

Aus Gärten zuweilen verwildert, so auch z. B. Sylt: Wemningstedt!!. Hierher gehört vielleicht als Rückschlag in den Typus $P h$. arundinacea von Helgoland, von Gätke in seinem Garten be- 
obachtet! (Knuth Fl. Helgol. 23 [1896], Ascherson Übersicht Helgol. $98[1900])$.

f. $m$. bractenta A. Christiansen nov. f. - Rispe mit entwickeltem Tragblatt mit Scheide und Spreite.

Schwansen: Schuby (A. Christiansen 1912!).

\section{Gattung.}

\section{Anthoxanthum.}

L. Gen. plant. ed. 5. 17 (1754) z. 'T.

1. Pflanze 4; Stengel nicht oder nur ganz am Grunde verzweigt; obere Hüllspelzen nicht doppelt so lang wie die Deckspelze ................... odoratum.

- Pflanze $\odot$; Stengel stark verzweigt; obere Hüllspelzen doppelt so lang wie die Deckspelze.

A. aristatum.

6. (sp. 3). Anthoxanthum odoratum L. spec. plant. ed. 1. 28 (1753).

4. Grundachse rasig. Stengel aufrecht, $(0,1-) 0,2-0,4(-1) \mathrm{m}$ hoch, glatt oder schwach rauh. Blattscheiden schwach rauh. Blattfläche in der Regel behart, $3-5(--10) \mathrm{mm}$ breit. Blatthäutchen $1-2(-3,5) \mathrm{mm}$ lang, gestutzt, am Ende oft zerrissen. Rispe $(2-) 3-6(-10) \mathrm{cm}$ lang, beiderseits verschmälert. Ährehen kurz gestielt. Untere Hüllspelzen ungleich, unterste bis $5 \mathrm{~mm}$ lang, gekielt, eimnerrig, zweite bis $9 \mathrm{~mm}$ lang, spitz, dreinervig, beide an den Nerven rauh oder gewimpert, selten behart. Obere Hüllspelzen fast gleich lang, die untere von ilmen aus der Spitze mit kurzer Grame, die obere mit längerer, das Ährehen oft überragender Grame aus dem Rücken der $3 \mathrm{~mm}$ langen Spelze. Deck- und Vorspelze 1,5-2 mm lang, gestutzt, glänzend. Staubblätter 2. Narben 2 am stielartig bis $1 \mathrm{~cm}$ verlängerten Griffel. Frucht $2 \mathrm{~mm}$ lang. Blüte Nai, Juni, einzeln bis Oktober.

An trockenen bis mälig feuchten, selten an nassen Orten, auf Wiesen, an Abhängen, in Wäldern und Gebüschen durch das Gebiet häufig; auch auf Sylt, Röm, Amrum, Föhr zerstreut oder nicht selten, zerstreut auf Helgoland: Oberland (Knuth!).

Für das Gebiet zuerst erwähnt ron Weber (Prim. Fl. Hols. + [1780]); wenig früher nemt sie O. F. Müller für das damalige Dänemark (Flora Danica t. 666 [1777]).

\section{Formen.}

1. Grame der obersten Hüllspelze die zweite Hüllspelze nicht oder wenig überragend. 
- Grame der oberstem Hëllspelze das Ihrehen weit (4-5) mme iöberragend :

f. longiaristatum Celakorsky Prodr. Fl. Böhmen 39 (1867).

Durch das Gebiet zerstreut, stellenweise (besonder's auf trockenen 'l'riften) in Menge. - L a u e n bu 1 ' $\mathrm{g}$ : Besenhorster Elbvorland in Menge!!. Hamburg: Warwisch!!, Horn (J.A.Schnidt 1884!, übergehend in f. umbrosum), Uhlenhorst (J.A.Schmidt 1865 ! bei f. villosum). Stormarn: zwischen Steinbek und Oejendorf und bei Wandsbek (J. A. Schmidt!), Ahrensfelde!! (bei f: villosum), Alt-Rahlstedt!! (bei f. strictum). Pinneberg: Holm!! (bei f. villosum). Lübeck: Wesloe!! (z. T. bei f. villosum, zu welcher Kombination $f$.lubecense P. Junge Jahrb. Hamb. Wiss. Anst. XXII. 60 [1905] gehört). Oldenburg i.H. : Neu-T'eschendorf!!, Heiligenhafen!! (hier auch bei f. villosum). Itzehoe: Schlotfeld!!. Ki el (A. Christiansen!). Flensburg: Glücksburg!!. Alsen: Bro!! (bei f. strictum). A penrade: Elisenlund!!.

2. Untere Hüllspelzen an (und oft auch zwischen) den Nerven mit langen Haaren . . . . . . . . . . . . . . . . . .

- Untere Hüllspelzen an den Nerven gezähnelt-rauh:

f. glabrescens Celakovsky a. a. O. 39 (1867), - Zerfällt in mehrere Formen ...................... 3

3. Blattscheiden kahl ........................ 4

- Blattscheiden sämtlich behaart:

f. silvaticum A.u.Gr. Synopsis Mitt.-Europ). Fl. II. 1.26(1898)²).

- Pflanze vielstengelig, groß; Blatter bis fast $30 \mathrm{~cm}$ lang.

Zerstreut, besonders an schattigen Orten. - L a u enburg: Hohenfelde(H.Röper!), Wentorf(J.A. Schmidt!). Hamburg (Kohlmeyer, Hb. Liibeck!): Bergedorf (J. A. Schmidt!), Berne (H. Röper!). Stormarn: Saselberg!!. Pinneberg: Wulfsmïhle!! L übeck: Schellbruch (Häcker!). Preetz: Kühren (A. Christiansen!), Probsteierhagen (A. Christiansen!). Kiel: Böhnhusen!!. Schleswig: Missunde (Nolte!). - Niedrige, kurzblättrige Ubergänge z. B. Ha m burg: Horn (J.A.Schmidt!), Storma rn : Togenkamp bei Wilstedt!!.

4. Sitengel und Blätrer schlatf: Rispenaste abstehend. daher Rispe sehr breit und locker:

f. umbrosum Bolle in Aschers. Fl. Brandenb. I. 802 (1864).

In schattigen (feuchten) Wäldern und Gebüschen verbreitet.

1) Identisch ist wahrscheinlich f. semiglabrum Schur Enumeratio Plant. Transsilr. 725 (1866). 
— stengel autrecht: Blätter küzer und starrer; Risje mit aufrecht anliegenden Ästen, daher ziemlich dicht:

f. vulgatum A. u. Gr. a. a. O. II. 1. 26 (1898).

Häufigste Form.

Dazu: f.giganteum P. Junge Jahrb. Hamb. Wiss. Anst. XXII. 60 (1905̃). - Stengel bis über $1 \mathrm{~m}$ hoch; Blätter bis $1 \mathrm{~cm}$ breit; Blatthäutchen verlängert, bis $9 \mathrm{~mm}$ lang. Rispe mit zahlreichen Ährchen, bis über $10 \mathrm{~cm}$ lang. Ährchen etwas größer als beim Typus.

Selten. - Stormarn : in einem Erlengehölz des Duvenstedter Brooks!!.

Eine Übergangsform nach dieser Pflanze hin bildet anscheinend f. majus Lange Haandbog Danske Fl. 3. Udg. 64 (1864); $f$. majus Hackel in F. Schultz Hb. norm. 1678 unterscheidet sich (vielleicht nur unwesentlich) durch gröbere Ährchen!.

5. Obere Blattscheiden kahl

- Alle Blattscheiden behart:

f. villosum Loisel. Not. pl. fil. franç. 7 (1810).

Durch das ganze Gebiet zerstreut. - Z. B. Lauenburg: Breitenfelde, Sachsenwald!!. H a m b urg: Rotenhaus!!, WVarwisch!!, Teddel (Zimpel!), Kuhwärder (Meyer, Hb. Beyle!), Uhlenhorst (Hansen!). Stormarn: zwischen Poppenbüttel und Langenhorn!!. Pinneberg: Bahrenfeld (Zimpel!), Niendorf und Wulfsmühle!!. Lübeck: Siems und Herrenfähre (Friedrich!). Angeln: Husby (Hansen), Flensburg: Marienhölzung (Prahl). Dithmarschen: Kuden (J.Schmidt!). Eiderstedt (Nolte!). Tondern: Hoyer (Friederichsen!). Föhr(Knuth). Amrum: Norddorf!!. Sylt: zwischen Munkmarsch und Kampen (Knuth), Westerland und Morsumkliff!!. Rö m: Toftum (Knuth), Lakolk (Jaap).

6. Stengel antrecht. kräftig; Blätter ziemlich loreit: Rispe dicht:

f. strictum A. u. Gr. a. a. O. II. 1. 26 (1898).

Zerstreut. - Lauenburg: Breitenfelde!!. Hamburg: Geesthacht!!. Stormarn: zwischen Trittau und Großensee!!, zwischen Horn und Schiffbek (J. A. Schmidt!), Hellbrook und Hummelsbüttel (Erichsen!), Wilstedt!!. Pinneberg: Langenfelde (Laban!). Lübeck: Bargerbrück!!. Preetz: Kühren und Kiel: Neumühlen (A. Christiansen!). Itzehoe: Föhrden-Barl!!. Sylt: Gr. Morsum!!. — Nach f. vulgatum leiten über Pflanzen mit ganz kahlen Scheiden, z. B. beobachtet Hamburg: Besenhorster Elbvorland und Apenrade: Runde Nühle!!. 
- Stengel anfsteigend, oberwäts überhängend, fein; Blätter schmal. voneinander entfernt; Rispe kurz, locker:

f. tenerum A. u. Gr. a. a. O. II. 1. 26 (1898).

Selten. -- Lauenburg: Bruchwald bei Fitzen!!.

\section{Mißbildungen.}

f. m. inipurm Aschers. Fl. Brandenb. I. 802 (1864). - Ährchen in Laubsprosse auswachsend.

Hamburg: Winterhude (A. Junge 1882!). Angeln: Grundhof (Hb. Kiel, anscheinend von Bargum, 1800!).

f. m. ramosum nov. f. - Rispenäste reichährig, sehr ungleich unregelmäBig stark verlängert, daher die Rispe ungleich lappig rerzweigt.

Ham burg: Bergedorf (.T. A. Schmidt!), Hammerbrook(.J. schmidt!), Hamm (Kausch!).

f. m. intrrontum nov. f. - Untere und zuweilen auch mittlere Rispenäste mregelmäbig stark rerkïzt, daher die Rispe ungleich mberbrochen.

Hamburg: Eppendorf (Zimpel!). - Eine Annäherungsform zeigt Reichenbach Icones Fl. Germ. et Helv. I. t. CLXXXII (1850).

7. (qs]). 1). Anthoxanthum aristatum Boissier Toyage Espag. II. (63s (1845).

$\odot$. Stengel $0,05-0,3(-0,4) \mathrm{m}$ hoch, am Grunde stark büschelig verzweigt, glatt oder sehr schwach rauh. Blattscheiden bei uns stets kahl, in der Regel glatt. Blattfliche kurz, 2-3 mm breit. Blatthäutchen 1-2(-3) mm lang, spitz, zerschlitzt. Rispe länglich. oberrärts schmäler. 2-5 cm lang, mit kurzen Ästen. Ährchen abstehend, bis $7 \mathrm{~mm}$ lang: Untere Hüllspelzen ungleich, unterste bis 3,5 mm lang, gekielt, (immervig, stachelspitzig, kilhl, zweite bis $6 \mathrm{~mm}$ lang, dreinelrig. gekielt. stachelspitzig, kahl. Obere Hüllspelzen fast gleich, etwa 2.5 mm lang, die untere von ihnen aus dem Spitzeneinschnitt mit 3 mm langer Grame. die obere vom Grunde des Rückens mit $7 \mathrm{~mm}$ langer geknieter tramne, beide behaart. Gramne die Hïlspelzen weit überragend. Deckspelze etwal $1,5 \mathrm{~mm}$ lang, kahl, glänzend, die Frucht umschliebend. Blüte Mai und Juni, zuweilen bis Oktober.

Auf sandigen Äckern und Sandfeldern sowie auf Schutt im sïdlichsten Teile des Gebiets in weiter Lmgegend Hamburgs nicht selten. eingebürgert; nordwärts an Häufigkeit abnchmend, im nördlichen Holstein sowie in Schleswig erst nenerdings selten beobachtet.

Ha mburg: neuerdings viel, zuerst: Hammerbrook (Laban 1866 in Hb. J. A. Schmidt!, A. Junge 187!)! แsw.). Kl. Grasbrook (Laban 1sti!). Hb. J.A. Schmidt!), Sternschanze (C. Timm 1869). Ẅinterhude (.J.A. schmidt 
1869 !), Barmbeck (J. A. Schmidt 1873 !). Chlenhorst 1874, Kulıwärder 1879 (C. Timm). Hamm 1879! und Horn 1882! (J. A. Schmidt): später häufiger, entfernter z. B. bei Bergedorf (Zimpel 1891!), Zollenspieker!!. St ormarn: znerst bei Wandsbek (W. Sonder 1873, Hb. Lübeck!), jetzt rerbreitet. Pinneberg: zuerst bei Schenefeld (C.Timm 1878: danach wohl Aschersons Angabe in Just. Bot. Tahresber. II. 1024 [1874]; aber Jahreszahl?), jetzt verbreitet. Lauenburg: Mölnn (Friedrich 1894!). Segeberg: Ulzburg: (Sack, Zimpel 1891 !), Kaltenkirchen!!. Lübeck: zwischen Burg- und Hüxtertor (K. Burmester 1912!). Ki el auf Schutt (A. Christiansen 1906 !). sichleswig (Ostermeyer). Flensburg: Sankelmark (A. Christiansen!)!!. Hadersleben: Starup) (A. Christiansen 1909!). Amrum (Jessen nach Engler Jahrb. XXVII. Beiheft 62. 6/7 [1899]).

Für das Gebiet zuerst erwähnt durch C. Timm (Verhandl. Nat. Ver. Hamb. N. F. IV. 69 [1880]) und Laban (Fl. v. Haml). usw. 3. Aufl. 17t [1877]).

Unsicher ist ein Vorkommen der Pflanze bei Lübeck. Eine bei Israelsdorf gesammelte Pflanze wurle von Garcke für A. Puellii erklärt, von dem Finder, Brehmer, aber als nicht. dazu gehörig bezeichnet. Das Exemplar im Lübecker Herbarium ist 1 . odoratum in einer Übergangsform nach f. villosum, gesammelt 1877 !. Indessen bielt Garcke nach Knuth (Fl. Schlesw.-Holst. 747 [1887]) an der Richtigkeit seiner Bestimmung fest.

\section{Formen.}

Nicht beobachtet.

\section{Gattung.}

\section{Hierochloë.}

R. Brown Prodr. Fl. Nov. Holland 208 (1810).

\section{8. (sp). 4). Hierochloe odorata Whlbg. Fl. Lusal. 32 (1820).}

4. Grundachse ausläufertreibend. Ausläufer etwa $2 \mathrm{~mm}$ dick. langgliedrig. glänzend. Stengel aufrecht, glatt. $(0,1-)(0.2-0,6(-0,8) \mathrm{m}$ hoch, oberwärts blattlos. Blattscheiden lang; sliatt. Blattfäche der Grund1) lätter gestreckt. der Stengelblätter kurz. Blatthäutchen 1-3 mm lang: stmupflich. Rispe meistens $6-10 \mathrm{~cm}$ lang. breit. locker. Ährchenstiele unter den Ährchen verdickt, kahl. Lntere Hüllsuelzen eiförmig, etwa 5) mun lang. glänzend, häutig. Obere Hüllspelzen mit je einer männlichen Blüte in ihrer Achsel, glinzend, am Rande gewimpert. etwa $5 \mathrm{~mm}$ lang, wenig länger als die schmale Vorspelze. Gramme ans der spitze der oberen 
Hüllspelzen sehr kurz, nicht oder nur wenig länger als die seitlichen Spitzen. Deckspelze der Zwitterblüte etwa + mm lang, länglich, gramnenlos. Mämnliche Blüten mit drei, Zwitterblüte mit zwei Staubblättern. Frucht ellipsoidisch, bis $2 \mathrm{~mm}$ lang. Blüte MIai und Juni.

Auf mäßig feuchten, seltener trockenen, Wiesen und Triften, an Flub- und Seenfern sowie in Hooren längs der kibe und Ostsee zerstrent, im mittleren Gebiet und im Westen selten, auf den Nordtriesischen Insehn nicht beobachtet.

Elbgebiet: Lanenburg: im Sachsenwald an der Aue im Rerier Ochsenbek (Hb. Lübeck ! ; ob ron Kohlmeyer?). H a m burg: anf Wiesen unterhalb der Hanmer Kirche (Hübener Fl. v. Hamb. 490 [1847]), zwischen Horner und Billwärder Park (A. Mohr 1903!), beim Mühlenkamp (Laban Fl. v. Hamb. 177 [1877]). Pinneberg: am Elbufer von Altona (J. J. Heyer 1814!) abwärts bis Wedel (Nolte, Hübener sen.! usw.), nenerdings nur unterhalb von Blankenese!!.

Ostseegebiet: Lauenburg (Meyer, Chloris Hannov. [1836i]): Wakenitzwiesen bei (irönau (Luther). Lübeck: Travewiesen unterhalb Lübeck mehrfach bis zum Schellbruch (Avé-Lallement 1821!, Häcker!, Friedrich! usw.), Neustadt (Schroedter 1831!): an der Burg (Rohweder). Oldenburg: Grömitz!!, viel im Oldenburger Bruch (Schultz, Hb. Kiel!)!! und am Wesseker See (Borchmann!) bis zur Brök!!. Kiel: mehrfach an der Kicler Föhrde von Heikendorf (Ohl 1891!) bis Bülk (Erichsen 1888!) (seit Bargum 1797! [?1791!]). Schleswig: Klensby (J. v. Schröder 1827, Paulsen, Himrichsen 1887!). Eckernförde: am Goossee bei Altenhof (A. Christiansen 1911!). Angeln: Beftoft (Bock 1890!), Langballigau und -holz (Hansen 1853 !). F le n s bu r gr: Quellental bei (ilïcksburg (Hansen!), Kielseng (Hansen), Clusries (M. T. Lange), Wassersleben (Prahl)!!, Küfermühlenhölzung (Poulsen usw.)!!. Alsen: am Miangsee (Petit, H. Petersen). A penrade (Bargum 1803!, Nolte 1825!): Runde Nühle bei Gjemner!!. Hadersleben: am Dam und an der Föhrde (steinvorth und r. FischerBenzon) z. B. Fredstedt (A. Christiansen 1907!).

Mittleres Gebiet: Hadersleben: am Untersee bei Jels (Prahl).

Nordseegebiet: Dithmarschen: am Fuhlensee bei Meldorf (J.Schmidt 1904!), auf Wiesen bei Hemnstedt (.J. Schmidt!), Hemmingstedt (J. Schmidt). Husum: am früheren Mühlteich (F. r. Müller). Rosenthal (r. FischerBenzon). Tondern: zwischen Tondern und Twedt (Hinrichsen!). Harrits (.J. Schmidt 1895!), zwischen Medolden und Ottersbüll (Borst). Hader'sleben: Gramm (Fabricius-Müller nach Bot. Tidsskr. 1. Reihe II. 38 [1867]).

Aus dem Gebiet zuerst erwähnt als Poa nitens von Webex (Prim. Fl. Hols. Suplel. 2 [1787]), und zwalr von I)ïsternbrook thier anch Nolte 1827 !, 1829!)。 


\section{Formen.}

f. pumila nov. f. - Stengel einzeln oder zu wenigen, 1-2 dm hoch; Blattfäche auch der Grundblätter kurz; Rispe kurz, wenigährig. Hungerform.

Pinneberg: auf Sandboden am Elbstrand bei Wittenbergen!!.

\section{Gattung.}

\section{Andropogon.}

L. Gen. plant. ed. 5. 468 (1754) erw.

\section{9. (adv. 4). Andropogon halepensis Brotero Fl. Lusit. I. 89 (1804).}

Ob bei uns 24? Grundachse kricehend, kurz, ansläufertreibend, bis $1 \mathrm{~cm}$ dick. Stengel aufrecht, bis 1,2 m hoch, glatt. Blattscheiden glatt. Blattfläche bis $20 \mathrm{~mm}$ breit, nur am Rande rauh. Blatthäutchen etwa $1 \mathrm{~mm}$ lang, am Rande fein kurzhaarig. Rispe langgestreckt, mit büschelig gestellten Ährchen. Ährchen zu dreien vereinigt, ein mittleres sitzendes mit Zwitterblüte und zwei seitliche, 3-4 mm lang gestielte mit münnlicher Blüte. Untere Hüllspelzen des mittleren Ährchens länglich-eiförmig, spitz, derb, glänzend; obere Hüllspelzen, Deck- und Vorspelze spitz, häutig. Deckspelze oft zweispitzig, mit bis $1,5 \mathrm{~cm}$ langer, geknieter, unterwärts gedrehter Granne. Männliche Ährchen etwa $6 \mathrm{~mm}$ lang, nit lanzettlichen, bräunlich-violetten Hüllspelzen. Blüte bei uns August bis Oktober.

Auf Schutt bei Hamburg und Kiel.

Hamburg: am Eppendorfer Noore (Laban 1892!), bei Wandsbek bei der Dampfmühle mehrfach (Zimpel 1894!, J. Schmidt 1897!) und bei der Helbingschen Brauerei (Zimpel 1897!), bei Bahrenfeld mehrfach 1905 und 1911!!. Kiel: Neumühlen (Prahl!).

Für das Gebiet zuerst erwähnt von Prahl (Krit. Fl. v. Schlesw.-Holst. $244[1890])$.

\section{Formen.}

f. ramifloru: nov. f. - Stengel aus den Blattscheiden rispentragende Äste treibend.

Hamburg: Bahrenfeld 1911!!.

6. Gattung.

\section{Zea.}

L. Gen. plant. ed. 5. 419 (1754).

10. (adv. 5). Zea mays L. Spec. plant. ed. 1. 971 (1753).

$\odot$. Stengel aufrecht, $1-2 \mathrm{~m}$ hoch, glatt, meistens einfach. Blatt- 
scheiden glatt. Blattfliche bei uns meistens $3-6 \mathrm{~cm}$ breit, glatt, am Rande kur gewimpert beharart, sonst in der Regel kahl. Bhatthäutehen ku\%, zerrissen. Blüten eingeschlechtlieh, die mämlichen in Ährchen in endstindiger Rispe, die weiblichen in Ihtehen an achselständigen spindeln in \%eilen ähnig angeordnet und von vielen Blattscheiden eingehüllt. Mämnliche ährehen 7 -s mm lang, mit breit-lanzettlichen, spitzen Hüllspelzen und lanzettlicher Ineck- mol Vorspelze. Stambblatter :3. Weibliche Ährehen meistens einblïtig, mit stmupflichen Hüllspelzen; Vorspelze wenig länges als die Deckispelzo. Fruehtknoten mit einer sehr lamgrestreckten Narbe. Frucht groß, glänzend. Blüte (Juli) August bis Oktober.

Auf Schutt und Gartenland nicht selten verschleppt oder verwildert, nur ansnahmsweise felderweise gebaut; oft nicht blühend.

\section{Gattung.}

\section{Tragus.}

Desfontaines Fl. Atlant. II. 386 (1800).

11. (adv. 6). Tragus racemosus Desfontaines Fl. Atlant. II. $386(1 \times()(1)$.

$\odot$. Stengel aus niederliegendem Grunde oberwärts aufsteigend, in fler Regel verzweigt, oft wurzehnd, glatt, $10-35 \mathrm{~cm}$ lang. Blattscheiden deutlich aufgeblasen. glatt. Blattlläche kurz. zuweilen fast fehlend. oft borstig bewimpert. Blatthautchen kurz, zerrissen, gewimpert. Rispe tranbenförmig. 4-5 cm lang. ziemlich dicht, ihre Aste meistens mit 3-5 Ährchen. Ährchen vom Rücken zusammengedrückt. Untere Hüllspelze klein, obere grof. siebennervig. auf den Verven mit widerhakigen Borsten besetzt. Deckspelze länglich, spitz. dreinervig. Torspelze länglich. spitz. Blüte Angust bis September.

Auf Schutt bei Hamburg.

Hatmburg: bei der Wandsbeker Dampfmühle (Kansch 1895 !, Laban!').

Aus dem Gebiete zuerst erwähnt im 4. Bericht des Bot. Ver. Hamburg (Deutsche Bot. Monatsschr. XIII. [1895]]).

\section{Gattung.}

\section{Paspalus.}

L. Systema ed. X. 855 (1759).

12. (adv. 7). Paspalus racemosus Lam. Ill. I. 176 (1791).

$\odot$. Stengel zu mehreren, aufsteigend, bis $0,6 \mathrm{~m}$ hoch, ästig, glatt. kahl. Blattscheiden glatt, kahl. Blattfläche $1-2 \mathrm{~cm}$ breit, glatt, am 
Rande schwach ratuh. Blatthäutchen fast fehlend. Risje 10-15 cm lang. mit seitlich gestellten. zu drei bis rier in gleicher Höhe entspringenden. (1-)1,こ-2 cm langen, abstehenden Ästen. Ährchen an den Ästen ährig gestellt. einseitswendig, zweireihig. $3 \mathrm{~mm}$ lang. einblïtig. Hüllspelzen 3) mm lang. einnerrig. spitzlich, bräunlich-häntig mit runzelig krausem Rancle. untere eiförmig-länglich. obere länglich. Deckspelze eifümiglänglich. $1.5 \mathrm{~mm}$ lang. stumpf. undeutlich genervt. glänzend. wie die fast gleich lange Torsuelze knorpelig-hart. Blüte Angust und September.

Auf Gartenland selten rerschleppt.

Helgoland: einmal in Gätkes Garten (Gätke, Hb. Helgoland!; Ascherson Wiss. Meeresunters. N. F. IV. 1. 19 [1900]) (Paspalum elegans nach Dalla-Torre Nat.-Med. Ver. Innshruck XTIII. 227 [1889] als ..mehrfach" beobachtet: P. megnus Flügge nach Kunth Allg. Bot. Zeit. IY. 139 [1898]).

\section{Gattung.}

\section{Panicum.}

L. Gen. plant. ed. 5. 29 (1754) verändert.

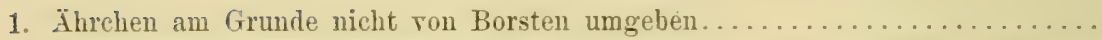

- ̈hrchen am Grunde von rauhen Borsten (grannenartig ausgebildeten Rispen-

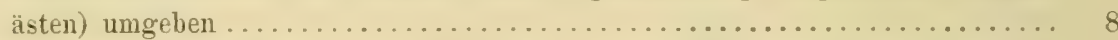

2. Ährchen in Ähren, welche sämtlich oder größtenteils fingerig gestellt sinŁ . 3

- Ährchen in Ähren, welche rispig stehen, oder einzeln an Stielen in Rispen... 4

3. Ährchen etwa $3 \mathrm{~mm}$ lang; untere Hüllspelze schüppchenartig, zweite Hüllspelze etwa halb so lang wie die dritte; Blattscheiden behaart......P. sanguinale.

- Ährchen etwa $2 \mathrm{~mm}$ lang; untere Hüllspelze an rielen Ährchen fehlend, zweite Hüllspelze wenig kürzer als die dritte; Blattscheiden in der Regel kahl. . P. lineare.

4. Ährchen in rispig gestellten Ähren......................... 5

- Ährchen einzeln, langgestielt, rispig gestellt .................. 6

5. Rispenäste stark rauh; dritte Hüllspelze stachelspitzig bis lang begrannt.

P. crus galli.

- Rispenäste glatt oder schwach rauh; dritte Hüllspelze spitz......P. colonum.

6. Pflanze 24, Blattscheiden kahl oder mit einzelnen Haaren......... P. repens.

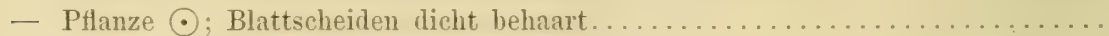

7. Ährchen langgestielt, etwa $4 \mathrm{~mm}$ lang................. miliaceum.

- Ährchen kürzer gestielt, etwa $2 \mathrm{~mm}$ lang................. capillare.

8. Ährchen $3 \mathrm{~mm}$ lang, gelbbraun mit quer gefurchter Deckspelze ...P. glaucum.

- Ïhrchen 2-2,5 mm lang, grünlich bis bräunlich, mit fein rauher Deckspelze..

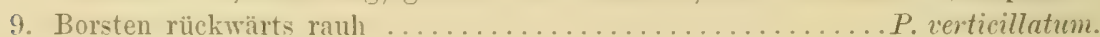

- Borsten vorwärts rauh . . . . . . . . . . . . . . . . . . . . . . . 10

10. Zweite und dritte Hüllspelze gleich lang ................ . viride.

- Zweite Hüllspelze etwas kürzer als die dritte ............. P. italicum. 3 4 . 6. 


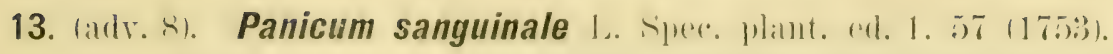

$\odot$. Stengel zahlreich, ans niederliegendem, oft wurzelndem Grunde

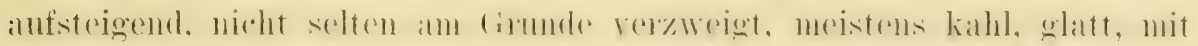

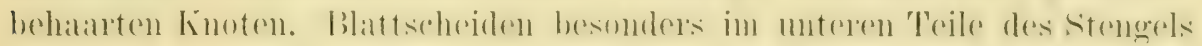
zerstreut bis dicht behaart. Blattfliche $4-11 \mathrm{~mm}$ breit, oberseits kahl oder sehwach behaart, unterseits dichter und länger behaart, mit weißlieben Randnerven. Batthäutrhen ku\%. zuweilen fast fohlend. Rispes

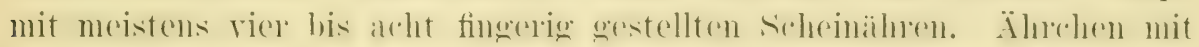

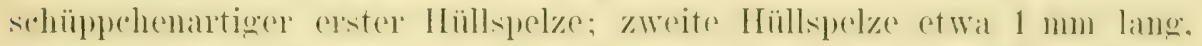

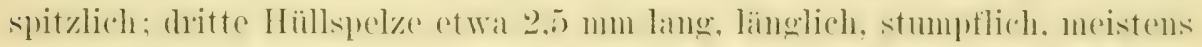

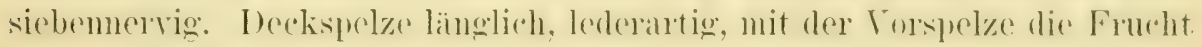
umschließend. Biüte Juli bis Oktober.

Auf Schutt und Gartenland, ausnahmsweise auch an WVegen und auf Äckern, selten und unbeständig durch das südliche Gebiet.

Lauenburg: Ratzeburg (Reinke). Stormarn: Reinbek (Sickmann, Laban), silk (sonder). steinbek mnd schiffbek (Häbenerr). bei der Mindsbeker Dampfmiihle (Zinpel 189.2!, 190()!). Ahrenshure (xomler). Hamburo

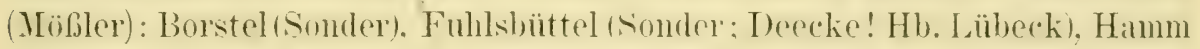
(Hübener, .T. A. Schmidt!), Horn (Hübener), Mühlenkamm) (Laban 1865!). Kl. Grasbrook (C. Timm, Zimpel!). Hammerbrook (A. Tunge). Linnebere: am Diebsteich in Altona (Laban. Erichsen 1s8s!). Bahrenfeld (Hübener)!!, Blankenese (Hübener[?]. Zimpel usw. 1891 !). L ü be cek: im Garten der Tonapotheke (Friedrich 18!5!). Ki e I : m Kleinbahnhof (A. Christiansen 1912!). Heide (Hennings!).

Aus dem Gebiete zuerst elwähnt von Mößler (Handb. Gewächskunde $79[1815])$.

P. sanguinale fand sich anscheinend früher öfter als in den letzten Jahrzehnten, wahrscheinlich infolge des früheren Anhanes. Sonder erwähnt ausdrücklich ihr Torkommen auf Äckem; Hübener nemnt sie häufiger als die folgende Art, was seine Angaben etwas fraglich erscheinen läßt. Nach Knuth (Fl. F. Schlesw.-Holst. 739 [1887]) soll Borchmann die Art bei Schöningstedt gefunden haben, doch fehlt sie in des letzteren Sammlung. Die Angabe ron Lübeck: Herrenfülure (Hücker, Lüb. Fl. 29 [1844]) bezieht sich auf $P$. lineare!; sie findet sich schon richtiggestellt durch Häcker selbst (Archir. Naturgesch. Mecklenb. XI. 135 [1857]). Ganz unsicher ist die Pflanze in dem Vermerk: bei Hohenwestedt an der Kellinghusener Chaussee nahe vor Lokstedt (Hemings, Schriften Nat. Ver. Schlesw.-Holst. II. 1. 145 [1876]). Wem Knuth a. a. 0.743 [1887]) für den Standort Heide C. Timm als Gewihhrsmann nennt, so beruht das auf einer Verwechslung. Derselbe Autor gibt (Schulfl. v. Schlesw.-Holst. 360 [1888] noch an „L." (Eutin) und „N." (Neumünster). Die erste Bemerkung geht auf Lienau (Phanerog. Pflanzen d. Fürstentums Lübeck 8 [1863]) zurück, der aber nur sagt: „Soll rorkommen“". Der Grund für die zweite Notiz ist mir unbekannt geblieben.

\section{Formen.}

f. repens A. u. Gr. Symopsis Mitt.-Europ. Fl. II. 1. 65 (1898). - Stengel sämtlich liegend, an den Knoten wurzelnd. 
Selten. - Hamburg: Kl. Grasbrook (Zimpel 1896!). Kiel: am Kleinbahnhof (A. Christiansen 1912!).

f. hirisutisimmm nor. f. - Stengel unter der Rispe mit zerstrenten, langen Haaren besetzt.

Selten. - Hamburg: Bahrenfeld 1900 !!.

An Formen ist weiter angegeben worden $f$. aegyptiacum Rebentisch, eine üppige, sonst aber durchaus vom Typus nicht zu tremnende Abart: H a m burg: an der Außenalster (C. Timm in Hb. J. Schmidt!, Knuth Fl. v. Schlesw.-Holst. 743 [1887]), auch gesammelt bei Bahrenfeld (A. Mohr!). Eine von Laban im Herbar als P. ciliare Trinius (Spec. gram. icon. XII. t. 144 [1829]) bezeichnete Pflanze gehört nicht dorthin!.

14. (q.1\%. 2). Panicum lineare lírocker Fl. Siles. 9s (1797).

$\odot$. Stengel in der Regel zahlreich, $(0,03-) 0,1-0,3(-0,5) \mathrm{m}$ lang; liegend oder anfsteigend, am (Arumde meistens verzweigt, glatt, mit kahlen Knoten. Blatscheiden fast stets kahl, glatt. Blattläche $(2-13-5 \mathrm{~mm}$ breit, nur am (ímude oft mit einem Hairbüschel, soust kahl. Blattläutchen kurz, gestutzt. Rispe mit $2-4(-12)$ Scheinähren. Ährchen meistens etwa 2 mum lang. Frste Hüllspelze sehr klein oder fehlend, zweite und dritte Hüllspelze gleich lang, letztere meistens tïnfnervig, beide zwischen den Nerven kurzhatrig. Teckspelze länglich, stumpflich. mit der Torspelze. die Frucht umschließend. Blïte Juli bis Oktober.

Auf Sandboden der Trege, Brachfelder und Äcker im südlichen (iebiet meistens häutig oder nicht selten, doch im nordöstlichen Holstein selten foder fehlend?), in sholeswig nordwärts seltener bis Flensburg: Handewitter Holzkrug (Lange 1846!), mehrfach bei Meyn (Prahl), Bommerlund (Poulsen), 'Tondern: Leck und Enge (P'rahl), Gallehums (stoltenberg; ob sicher?). Auf den Nordfriesischen Inseln nur Föhr: Nieblum (Schiötz).

Aus dem Gebiet zuerst erwähnt bei Mößler (Handb. Gewächsk. 79 [1815]). Die Pflanze erscheint bei Hornemamn (Bemaerkninger angaaende Forskelligheden af Tegetat. Damsk. Prov. 194 [1821]) als Symtherisma glabrum (!). Zu ihr gehört wohl auch Digitaria sangumea (Weber Prim. Fl. Hols. 6 [1780]), wie sicher Panicum sanguinale Oeder (Flora Danica 388 [1768]). Das älteste gesehene Exemplar des Gebiets wurde in Angeln: zwischen Süderbrarup und Brebel 1808! gesammelt.

\section{Formen.}

1. Blattscheiden völlig kahl.................... 2

- Blattscheiden zerstrent kurzharig:

f. hirtum nov. f. - Obere Blattscheiden kahl, untere mit bis $1 \mathrm{~mm}$ langen Hürchen.

R endsburg: Hohenhörn!!. 
2. Stengel wenig verzweigt; Blattscheiden anliegend; Scheinähren $(1-2-6 \mathrm{~cm}$ lang, zu $2-4 \ldots \ldots \ldots \ldots \ldots \ldots \ldots \ldots \ldots \ldots \ldots$

- stengel reich rewzweigt, oft wuzelud: Blattseheiden weit; soheinähren 4-8 cm lang, zu 6-12:

f. prostratum A. 11. Gr. Synopsis Mitt.-Europ. Fl. II. 1.67(1898). Selten. - Stormarn: Ladenbek bei Boberg!!. Lïbeck: Herrenfähre (Häcker). - Übergang: La u en burg: Rotenhaus (Lange!).

3. Ährchen genähert, etwa $2 \mathrm{~mm}$ lang: Typische Form.

- Ährchen etwas entfernt, (1-)1,5 mm lang:

f. gracillimum A. u. Gr. a. a. O. II. 1. 67 (1898). - Pflanze niedrig. Stengel wenig zahlreich, fadenförmig, $3-10 \mathrm{~cm}$ lang, mit $1-2(-3)$ Scheinähren. Blätter schmal, kurz. Selten. Lauenburg: zwischen Pötrau und Witzeeze!!. Kiel: am Flintbeker Moor (A. Christiansen!).

15. ((1)1. 3). Panicum crus galli L. Nipece plant. ed. 1. כ6 (1753).

$\odot$. Stengel $(0,1-0,3-0,8(-1) \mathrm{m}$ lang, aufrecht oder aufsteigend, in der Regel einfach, glatt, an den Knoten kahl. Blattscheiden glatt, kahl. Blattfläche kahl, oberseits und am Rande rauh, 4-15(-20) mm breit. Blatthäntehen tohlend. Rispe ans rispig gestellten sicheinähren gebildet, die am Grumde Harrbüschel tragen, mit stark rauhen ästen. Ährohen spitz-eifomig. gexen $4 \mathrm{~mm}$ lang. Hrste Hüllspelze bis 1 mm lang. breit-eiformig, slitz, dreinervig. \%weite Hüllsuelze 8.5 mm lang. spitz, zuweilen mit kurzer dirame. füntnervig. Dritte Hüilspelze so lang wie die zweite, spitz, kum bis lamg begramut, siebemervig. wie die zweite auf dem Rücken körnig, am Rande länger und derber behaart, in der Achsel eine bis auf die Torspelze fehlschlagende Blüte tragend. Deckspelze etwa 2,5 mm lang, unbegrannt, kahl, glänzend, die Frucht umschließend. Blüte Juli bis Oktober.

Auf Garten- und Bage Liand, auf Äckern und Schutt, weniger auf ptlanzenamem Boden an Fläsicen. Teichen und in firäben: nicht solten

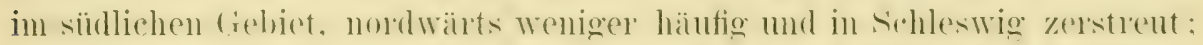

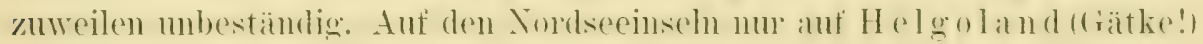
und anf Föhr: Nieblum!! als Kartoffelunkraut.

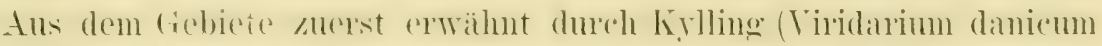

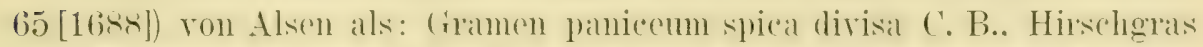
mit gespaltenen Ähen. D)ie Pllanze wurde atuf Alsen nenerdings nur bei sonderburg gesimmelt 1H. Petersen Programm Realsch. Sonderb, 4:3 [1891]). 


\section{Formen.}

1. Initg Hüllsperze mit langer (trame (in der Regel üher $10 \mathrm{~mm}$ lang): f. Tongisetum Döll. Fl. v. Baden I. 232 (1857). - So anscheinend seltener.

- Initer Hüllspelze kurz begrament oder nur stachelspitzig. nur an den Endährchen der Scheinähren zuweilen länger begrannt:

f. trevisetum Döll. Fl. v. Baden I. 232 (1857). - So häufiger. Dazu:

f. panciflom A. u. Gr. Synopsis Mitt.-Europ. Fl. II. 1. 68 (1898). - Pflanze niedrig; Rispe klein, locker; Rispenäste wenigälırig. Blätter schmal.

Hamburg: zwischen Barmbeck und dem Hinschenfelder Holz (J. A. Schmidt!). - Übergangsform: It z eh o e (Nolte 1822!; z. T.).

f. ramiflorm nov. f. - Stengel aus allen Knoten mit rispentragenden Ästen.

Hamburg: Wrinterhuder Bruch, Harrestehude, Georgswärder (Zimpel 1890!, 1892!, 1893!).

16. Gartr. 41. Panicum colonum L. sirtema ell. 10. 8701175911.

$\odot$. Stengel $0,2-0,3 \mathrm{~m}$ hoch, aufrecht oder aufsteigend, einfach,

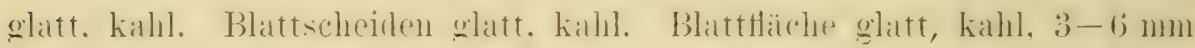
breit. Blatthäutchen fehlend. lisise mit rispigg gestrolten soheinähren.

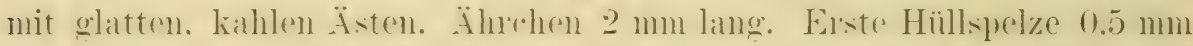
lang. mit starkem Ditteherr. Zweite und dritte Hüllspelze greirh lang. erstere dreinervig. letztere fünfnervig. beide ant dem Rë̈cken behatrt. spitzlich. mbegrannt. Deckspelze glatt. kamm glänzend. Blüe August bis Oktober.

Auf Schutt bei Hamburg.

Hamburg: auf Schutt (Kaffeeabfällen) bei den Aitonaer Wasser-

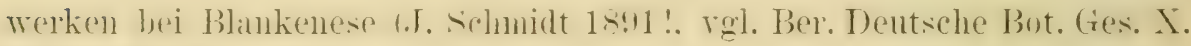
86 [1892], Zimpel!).

17. la(15. 10). Panicum miliaceum L. sper. plant. ed. 1. 58 (1753).

$\odot$. Stengel $(0,2-) 0,5-1 \mathrm{~m}$ hoch, aufrecht oder aufsteigend, einfach orler moterwärts ästig. behant oder oherwärts kahl. Blattscheiden weich-

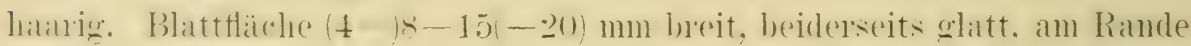

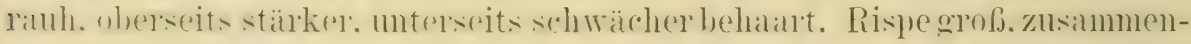
grestzt. Ährchen rinzeln, langgestielt. an ranhen Ästen. hellgrün oder brämulich-rislett. otwal 4 mm ling. Erste Hülls grelze 2.5 mm lang. zweite 
und dritte Hüllspelze $4 \mathrm{~mm}$ lang, alle dentlich nervig, spitz, kahl. Torspelze an der dritten Hüllspelze kum, eiförmig. Deck- mud Torspelze der Zwittroliite gleich. :) mun lang. gelblich, kahl, glänzond, die Frucht umschließend. Blüte Juli bis Oktober.

Auf Schutt und Gartenland, seltener auf Äckern, verschleppt.

L a u e n burg: Aumühle!!, Kuddewörde!!. H a m burg : nicht selten, aber unbeständig, frïher nach Häbener (Fl. Y. Hamb. 4se [1S47]) in (iärten gepflanzt und verwildert. Lä̈berk: zuweilen anf sehutt. (Friedrich), bei Ratekau und Pansdorf (Ranke 1893!). Kiel: Gaarden (A. Christiansen 1906!). Hadersleben: Stepping (Prahl). 'Tondern: Medolden (Borst). Aus dem Gebiete zuerst erwähnt von Hübener (a. a. O. 488 [1847]).

\section{Formen.}

f. ramiflorum nov. f. - Stengel aus den oberen Knoten mit rispentragenden İsten.

Selten. - Hamburg: Fuhlsbüttel 1912!!.

18. (adv. 11). Panicum capillare L. Spec. plant. ed. 1. 5s (1753).

$\odot$. Stengel $0,3-0,6 \mathrm{~m}$ hoch, aufrecht oder aufsteigend, aus den Knoten oft beästet, muter den dichtharigen Knoten behaat, sonst kahl. Blattscheiden dicht weichharrig, ihre Hatre aufrecht abstehend. Blattfläche oberseits an den Verven kurzharig. unterseits zerstrent kmzharig. am Rande rauh. Rispe reichästig, bis $30 \mathrm{~cm}$ lang: Ährchen einzeln, meistens kurzgestielt, bis 2 $11 m$ lang, länglich, spitz. Erste Hüllspelze breit-eiförmig, mit kurzer Spitze, etwa $1 \mathrm{~mm}$ lang, dreinervig. Zweite und dritte Hüllspelze fast gleich lang, spitz, kahl, fünfnervig. Dritte Hüllspelze olne Torspelze. Deckspelze 1,5 mm lang, glänzend, kahl. Blüte Juli bis Oktober.

Bei Hamburg und Lübeck verschleppt.

H a m bur $\underline{g}$ : ()ejendorf (.J. Schmidt 1896!). Wandsbeker Dampfmühle

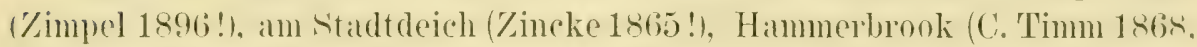
.J. Nehmidt 1889!), am Hasiclbrook in Hamm (.T. A. Sechmidt 1865!). Hor'n (A. Junge 1880). Uhlenhorst mehrfach (A. Junge 1875!. Katusch 1654!). Winterhude 18ig und spaiter (C. Timm, A. Jumge!, J. Schmidt!, Zimpel!), beim Eppendorfer Moore (Laban), zwisehen Bambeck und steilshop (Zimpel 1891!), steinwärles (C. Timm), beim Altonaer Bequabnisplatz (Laban.

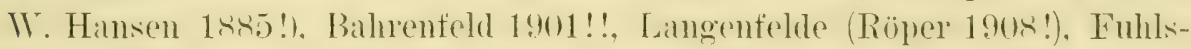

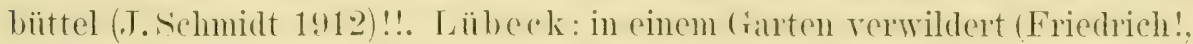
Deutsche Bot. Ges. X. 87 [1892]).

Die erste Erwähnung aus dem Gebiet findet die Art durch C. Timm (Terhandl. Nat. Ver. Hamb. N. F. II. [1878]). 


\section{Formen.}

f. gracillimum A. u. Gr. Synopsis Mitt.-Europ. Fl. II. 1. 72 (1898). Ahrohmstiels sehre gestreckt, an den endständigen Ährchen bis $5 \mathrm{~cm}$ lang.

Hamburg: Dampfmühle Wandsbek (Zimpel 1896!).

19. 4adv. 12). Panicum repens L. Suer. plant. ed. 2. si $(1762)$.

4 (ob auch bei uns?). Stengel $0,2-0,5 \mathrm{~m}$ lang, liegend oder aufsteigend. zu mehreren. unterwärts oft stalk ästig. glatt. kahl. Blattscheiden glatt, kahl, oft riolett überlaufen. Blattfläche $3-6(-8) \mathrm{mm}$ breit, am

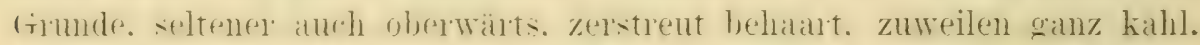
Blatthäutehen als Hadreile entwickelt. Rispe locker. mit einzeln oder

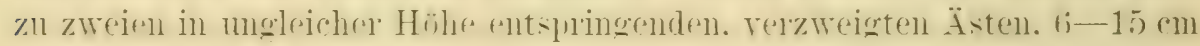
lang. Ährchen an kurzen Ästen, 2,5 mm lang, eiförmig, spitz. Erste Hüllspelze breit. 11.5 mm lang. Zweite und dritte Hüllspelze 2.5 mm lang. slitz. kahl. Derk- und Vorspelze 2 mm lang. olänzend. Blüte Augrat bis Oktober.

Auf- Schutt bei Hamburg selten.

Hamburg: bei der Dampfmühle Wandsbek (A. MIohr 1901!).

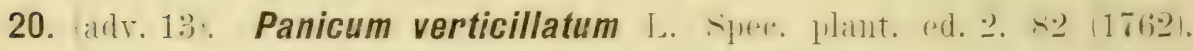

$\odot$. Stengel $0,3-0,8 \mathrm{~m}$ lang, aufrecht oder meistens ans liegendem frumde ant-teigend. muter der Rispe anf kuzer strecke rauh. sonst glatt, einfach oder am Grunde verzweigt. Blattscheiden in der Regel glatt,

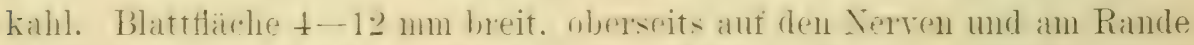

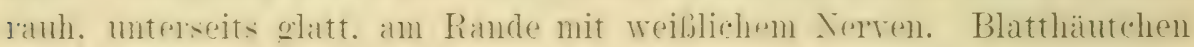
kurz, wimperhaarig. Rispe $5-10 \mathrm{~cm}$ lang, an der Spitze etwas ver-

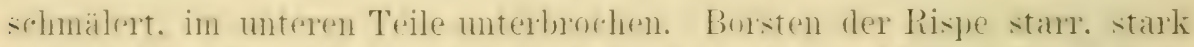
rǘckwärts rauh, selten vorwärts rauh. his 7 mm lang. Ährehen 2 mm lang.

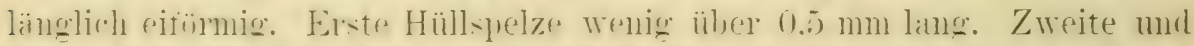

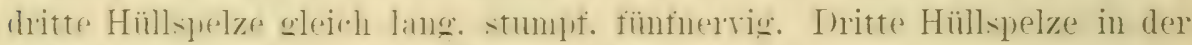

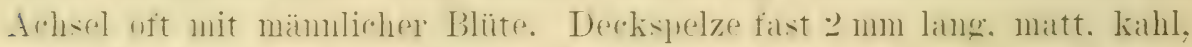
mit Zwitterblüte. Blüte Juli bis September.

Auf schutt und Gartenland selten.

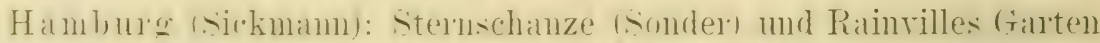

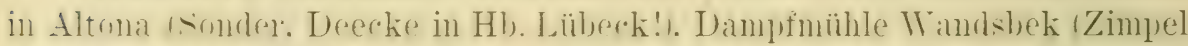

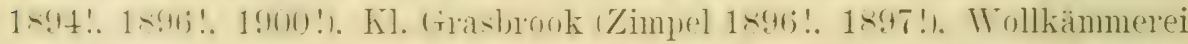

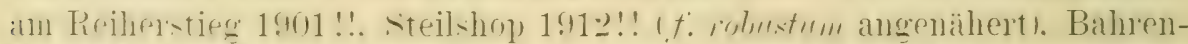

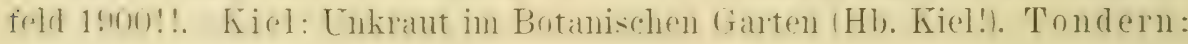
Deezbüll (Jörgensen 1840 !). 
Dic erste Erwihnmo findet die Art durch Nolte Novit. Fl. Hols.

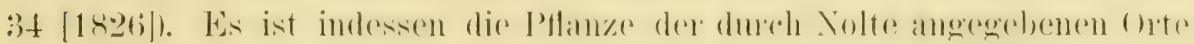
nach Ausweis seines Herbars Panicum vivide: der Standort im Botanischen

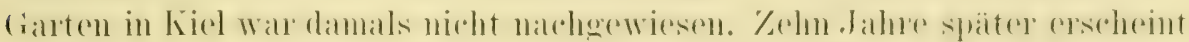
die sickmammsche Notiz (Emmmeratio Hamb. A |l sobil). Anch sie ist nicht über allen '/weifel erhaben. Richtig ist aber die Angabe Hornemanns (Flora Danica t. 2063 [1836]), insoweit der Standort 'Tondern in Frage kommit.

Nach Sonder (Fl. Hamb. 38 [1851]) hat Sickmamn P. verticillatum ron Reinbek, Barmbeck und Wohldorf angegeben. Exemplare sah Sonder nicht. Er erwähnt auch Wellingsbüttel als Fundort, und zwar nach Angabe der Llora Danica (t. 2063 [1836]). Noltes Pflanze von dort ist $P$. viride (Hb. Kiel!). Dasselbe gilt von einer Pflanze von Molln (Hb. Kiel!). Trotzdem hat Knuth, der beide Exemplare gesehen hat, sie als P. verticillatum aufgenommen (Fl. v. Schlesw.-Holst. 744 [1887]). Auch der an der gleichen Stelle aufgezïhlte Standort Witzhave (Borchmann) bezieht sich auf P. vivide!. Dasselbe dürfte für die Lübecker Lrwähnungen unserer Art gelten, die Knuth (a. a. O.) un eine vermelurt hat: Vor dem Holstenthor (Hansen). Völig unverständlich ist es, wenn es bei Laban (Fl. v. Holst. 221 [1866]) heißt: "Um Lübeck läufig". Richtig ist nur der 'Zusatz: „.. sonst selten". Nicht belegt sind die beiden folgenden Bemerkungen: Rendsburg: Hohenwestedt, Versuchsfeld der landwirtschaftlichen Schule unter Getreide (Giersberg nach Hennings Nat. Ver. Schlesw.-Holst. II. 1.145 [1876]) und Dithmarschen: an Wege nach dem Wöhrlener Hafen (bei Heide) (Siercks und Henningsen, Handschriftl. Verz. nach Kinuth a. a. 0.). Auch hier dürfte $P$. vivide die Grundlage der Angabe bilden.

Die Kieler Pflanze führt ohne Findervermerk die Notiz: „Unkrant im Garten. Vermutlich mit fremden Sämereien eingeführt." Es dürfte zweifellos der Kieler Botanische Garten gemeint sein. Von hier stammt vermutlich die von Hansen ausgegebene Pflanze.

\section{Formen.}

f. ambiguum Gussone Prodr. Fl. Sic. 80 (1827). - Borsten die Ährehen

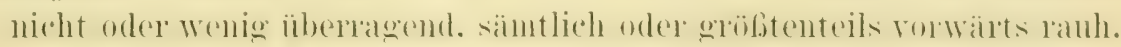
H a m burg: Dampfimühle Wandsbek (A. Jiohr 1901!).

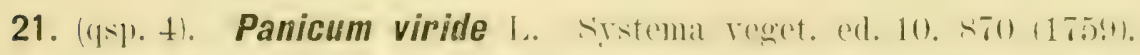

$\odot$. Stengel $(0,3-) 0,1-0,4(-i)$ m hoch, in der Regel aufrecht oder aufsteigend, verzweigt (oft stark), nur unter der Ahre schwach latuh, sonst glatt. Blattscheiden oglatt, nm anter der Mïndume am Ramde gemimpert. sonst kahl. Blattfläche kahl. oberseits und am Rande raul. unterseits glatt, 3-10 mm breit. Blatthäntchen fast fehlend, mit starkem Haarkranz. Rispe $(1,5-) 3-6(-10) \mathrm{cm}$ lang, dicht, nicht gelappt, meistens 6-8 mm breit. Borsten düm, biegsam, vorwärts rauh, bis $10 \mathrm{~mm}$ lang: Ähchen $2 \mathrm{~mm}$ lang, oval. Erste Hiilspelze nicht halb so lang wie das

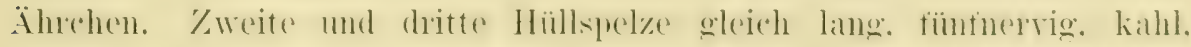

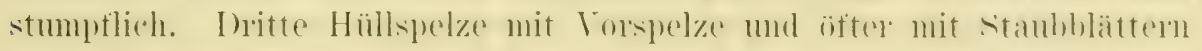


Ansatz zu zweiter Blütel. Treck- und Torselze gleich. etwa $2 \mathrm{~mm}$ lang. Blïte Juni bis Oktober.

Auf Äckem, Brachfeldern und Gartenland, auf Schutt und Baggererder sowie an Wegem im südlichen trebjet häutig order nicht seltem. im

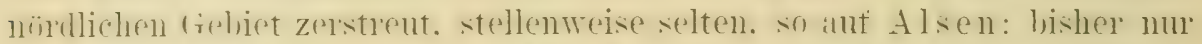

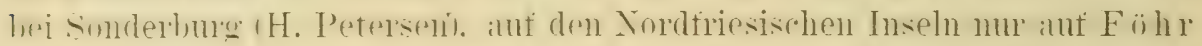
selten (Schiotz) und auf Amrum (Jessen nach r. Seemen); ferner auf Helgoland (Gätke 188t!).

Die erste Angabe aus dem Gebiete steht bei Weber (Prim. Fl. Hols. 6 [1780]).

\section{Formen.}

1. Stengel 3-40 cm hoch; Blätter ziemlich schmal; Rispe bis $6 \mathrm{~cm}$

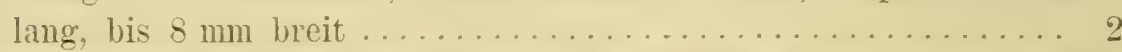

- Stengel $60-100 \mathrm{~cm}$ hoch; Blätter bis $10 \mathrm{~mm}$ breit; Rispe bis $10 \mathrm{~cm}$ lang, 8-10 num breit:

f. majus Gaudin Fl. Helv. I. 152 (1828).

Selten. - H a mburg: Winterhude (C. Timm)!!, Bahrenfeld!!. Helgoland (Gätke!). - Übergänge zerstrent.

2. Stengel meistens $20-40 \mathrm{~cm}$ hoch, aufrecht, selten liegend:

f. rechinatum A. u. Gr. Synopsis Mitt.-Europ. Fl. II. 1. 77 (1898). Häufigste Form.

- Stengel $3-5(-10) \mathrm{cm}$ hoch, aufrecht oder seltener liegend:

f.pygmaeum A. u. Gr. a. a. O. II. 1. 7 (1898).

Auf trocken-sandigem Boden zerstreut, stellentreise in Menge.

\section{Farbenformen.}

f. Teimmanni A. u. Gr. a. a. O. II. 1. 77 (1893). - Ährenborsten und Hüllspelzen violett überlauten.

So hin und wieder, besonders bei $f$. pygmaeum.

\section{Mißbildungen.}

f. m. furcutum nor. f. - Rispenachse oberwärts dreiteilig, Rispe daher dreispaltig.

Hamburg: bei der Sierichbrïcke in Winterhude (Zimpel 1893!).

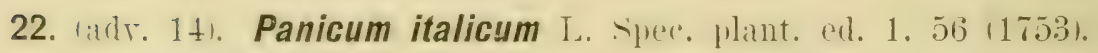

$\odot$. Stengel $0,5-1,2 \mathrm{~m} \mathrm{hoch}$, in der Regel verzweigt, meistens aufrecht, nur unter der Rispe rauh, sonst glatt. Blattscheiden oberwärts am Rande wimler hatarig. sonst in der Regel ganz kahl. glatt. Blattläche $(6-) 8-15 \mathrm{~mm}$ breit, oberseits schwach rauh, unterseits sehr schwach 
rauh bis glatt, am Rande stark rauh. Blatthäutchen fast fehlend, wimperharig: Rispe (5-)10--15 cm lang, bis $15 \mathrm{~mm}$ breit, dicht, in der Regel dentlich gelappt. Borsten ziemlich fest, vorwärts rauh, bis

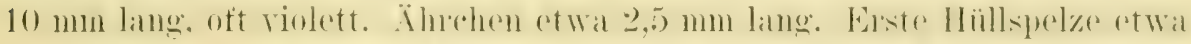

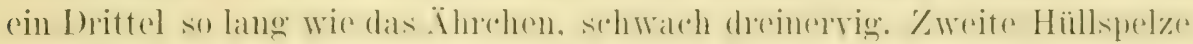

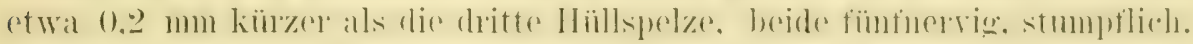

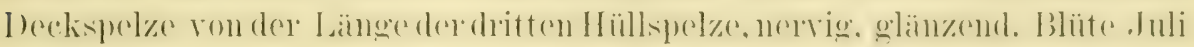
bis Oktober.

Auf Schutt und Gartenland selten.

Lauenburg: bei Krïmmel 1911!!, bei Aumiihle (Laban 1884!).

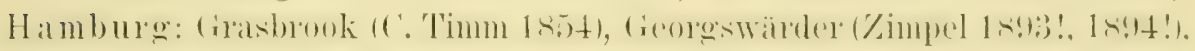
Steinwärder (C. Timm 1859, Laban 1871!), St. Panli (C. T'imm 1859),

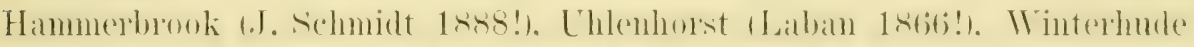

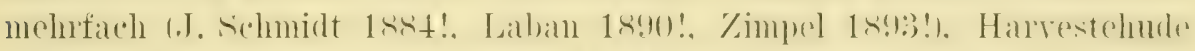
(\%impel 18!)!). Barmbeck (Boyle 1893!). Fublsbüttel 1!12!!. Holsten-

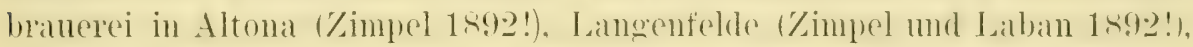
Niendorf 1897!!. Lübeck (Häcker, nach Angabe im Herbar kultiviert!) ant fichutt (nach Friedrieh). Kiel (Prahl. A. ('hristiansen 1!n)!). Flensburg (Prahl).

Die erste Erwähnung der Art aus dem Gebiet findet sich dureh C. 'T. 'I'imm.

Formen.

f. longisetum Döll Fl. v. Baden I. 233 (1857). - Borsten mehrmals länger als die Ährchen.

So häufiger.

f. gemanicum Lam. u. DC. Fl. France III. 14 (180כ̃). - Borsten wenig länger als die Ährchen.

So seltener. — Ha mburg: Georgswärder (Zimpel!), Winterhude (Laban 1890!), Wandsbek (Zimpel!). Kiel und Flensburg (Prahl).

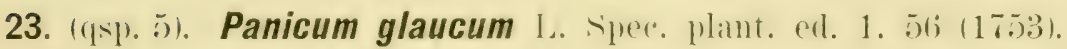

$\odot$. Stengel $(0,05-) 0,1-0,3(-0,6) \mathrm{m}$ hoch, aufrecht oder aufsteigend bis niederliegend, nur unter der Rispe auf kmzer Strecke rauh, sonst glatt. Blattscheiden kahl, glatt. Blattläche meistens $4-6 \mathrm{~mm}$ breit, oberseits schwach ranh, unterseits glatt, am Rande schwach raul und gegen den Blattgrund lang wimperhatrig wir der Grumd dex Blattoberseite. Blatthäutchen nur als Reihe kurzer Härchen vorhanden. Rispe $(2-) 3-5(-8) \mathrm{cm}$ lang, 6-8 mm breit, dicht, ungelappt, zylindrisch.

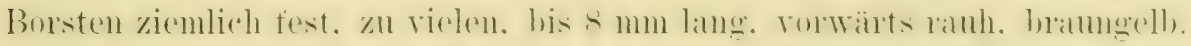
Ährchen $3 \mathrm{~mm}$ lang, etwa $2 \mathrm{~mm}$ breit, auf einer Seite fast flach, auf der

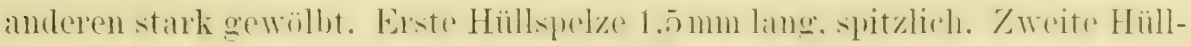


spelze $1.5 \mathrm{~mm}$ lang, stumpflich, fünfnervig, viel kürzer als die Deckspelze. Initte Hüllspelze so lang wie das Ährehen, eimnervig. Deckspelze so lang wie das Ährehen, stark gewölbt, quermulig. Vorspelze flach, schr schwach querrunzlig. Frucht fast halbkugelig, $2 \mathrm{~mm}$ lang. Blüte Juli bis Oktober.

Auf Äckern sowie auf Schutt, Baggererde und Gartenland im südöstlichen Giebiet bis Hamburg-Lübeck zerstrent, stellenweise nicht selten, nordwärts durch Holstein selten, in Schleswig anscheinend bisher nicht beobachtet.

Lauenburg: Lanenburg (Clandius), Lanze (Klatt), viel bei Bröthen, Fitzen und Bergholz!!, Oberschlemse! und Horst bei Möln (Nolte), Ratzeburg (Nolte nach Flora Danica t. 2162 [1836i]), (ir. Disnack (Franz Thorn 1911!), (irönan (Luther), Escheburg!!. Stormarn: Reinbek (J. J. Мeyer 1816!, Sickmamm, Witzhave (Borehmann), Trittan (Thun 1^33!), Steinbek (C. Timm), Wandsbek (Hübener)!!. Poppenbüttel (A. Mohn!), am Bahnhof Reinfeld (Ch. S'onder). Hamburg: Harrestehule und Eppendorf (Möbler; vor 1815). Borstel (Sickmann; vor 1836), Horn (Hübener; 1847), Hammerbrook (Laban 1865!). später vielfach (!. !!). Pinneberg: Nienstedten (Hübener), Flottbek (Sonder, Deecke! Ho. Lübeck), Blankenese (Sonder). Lü̈beck: hier und da (Hätcker), z. B. Beidendorf (Häreker!), zwischen Dummersdorf und Herrenwiek (Griewank 185:3!, P'rahl), aut schutt nicht selten (Friedrich, mehrfach!). Eutin: Gleschendorf (Franz Thorn 1911!). Segeberg: Gönnebek (Prahl). Preetz: Rastorfer Passau (Prahl). Kiel: Neumühlen (Erichsen 1887!). Bordesholm (J. Lange 1847!). Itzehoe: Ridders und Lohmühle beim Lockstedter Lager (Prahl).

Die erste Erwähnung aus dem Gebiete findet die Art bei Mößler (Handb. Gewächskunde 112 [1815]).

Ans Schleswig findet sich eine Angabe des Grases von Schleswig: Brodersbye (J. v. Schröder in Geschichte und Beschreibung der Stadt Schleswig 398 [1827]), die vermutlich auf Esmarch zurückgeht. Ol) sie begrumdet ist oder nicht, ist heute nicht mehr festzustellen, die Richtigkeit aber ist nicht gerade wahrscheinlich. Unbestätigt ist die Notiz bei Lienau (Phanerog. Pfl. Fürstentum Lübeck 8 [1863]): „Nach der Erndte hier und da". Dasselbe gilt vou einer Bemerkung bei Hennings (Schriften Nat. Ver. Schlesw.Holst. IV. 95 [1880]): "auf einem Acker bei Ellerbek". Knuth nennt (FI. v. Schlesw.Holst. 745 [1887]) Rotenhaus (bei Bergedort) als Fundort, Nolte als Sammler. Die betreffende Pflanze ist $P$. vivide!.

\section{Formen.}

f. memilum A. u. Gr. Synopsis Mitt.-Europ. Fl. II. 1. 79 (1898). - Pflanze 4-8 cm hoch; Rispe wenigährig, kurz.

Lauenburg: Bröthen, Bergholz, Escheburg!!. 
Panicum concinnem "L." ist ein bei Hamburg: Georgswärler beobachtetes Gras genannt worden (vgl. Deutsch. Bot. Monatsschr. XVI. 115 [1898]); Belege sind anscheinend nicht mehr vorhanden, so daß eine Feststellung der Zugehörigkeit der betreffenden Pflanze ummöglich ist.

Pennisetum Rich. in Persoon Syn. I. 72 (1805) ist im Gebiete in leiner Art nachgewiesen: eine Angabe ron ,Pennisetum sp.“ für Hamburg: Wollkïmmerei am Reilierstieg (Zimpel nach Deutsch. Bot. Monatssehr. XVII. [1899]) ist nicht belegt, so daß weder Art noch Gattung des betreffenden Grases festzustellen ist.

10. Gattung:

\section{Cenchrus.}

L. Gen. plant, ed. 5. 470 (1754).

24. (alle. 15). Cenchrus tribuloides L. Suece plant. ed. 1. 1050 117531. C. Stengel 0.2-0.t m lang. ans niederliegendem Grunde anfisteigend. aus den Knoten verzweigt, oft wurelnd, glatt. Blattscheiden weit ab-

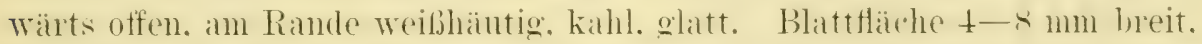
oberseits schwach rauh, unterseits glatt. Blatthäutchen bis auf einen dichten Kranz kurzer Haare fehlend. Rispe 4-8 cm lang, etwa $5-9 \mathrm{~mm}$ breit, unterbrochen. Ährchen von dichtstachliger Hülle mit rïckwärts latuhen stacheln nmgehen. zn $1-3(-4)$. lanzettlich. Hïllspelzen ungleich

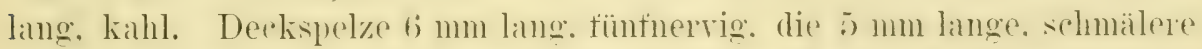
Vorspelze und die Frucht umschliefend. Blüte August bis Oktober.

Auf Schutt bei Hamburg selten.

Hamburg: bei den Altonaer Wasserwerken bei Blankenese (IV. Timm

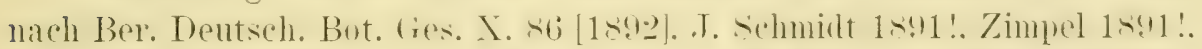

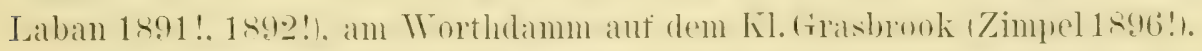

Die erste Erwähnung für unser Gebiet findet die Pflanze im 3. Ber. Bot. Ter. Hamb. (Die Heimat IV. 8 [1894]) nach rorherig’er Nennung im Bericht der Deutschen Bot. Gesellschaft.

\section{Gattmin:}

\section{Cynodon.}

Richard in Persoon Syn. I. 85 (1805).

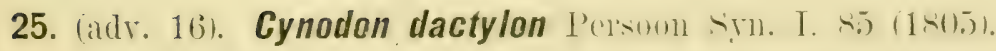

4. Grundachse lang gestreckt, an den Knoten Ansläufer und (oder) aufrechte oder anfsteigende. an cirumde ötter wuzelnde stengel treibend. 
Stengel oft nur als Lanultriche entwickelt. $0,1-0,4 \mathrm{~m}$ hoch, glatt. Blattscheiden weit geöffnet, glatt, kahl. Blattfläche glatt, beiderseits oder muterseits behart, seltener kahl, am Rande in der Regel rauh, 2- $4 \mathrm{~mm}$ breit. Blatthäutchen kurz, mit Haarkranz. Rispe mit $3-6$ fingerig gestellten Ähren. Ähren 2-7 cm lang, mit einseitig gestellten Ährehen. Ährehen einblütig, zuweilen mit verlängerter Achse, $2 \mathrm{~mm}$ ling, mit zwei Hüllspelzen. Hüllspelzen 1,5 mm lang, spitz, eimnervig, gekielt, am Kiel gewimpert. Deckipelze mit gewölbtem, kuz gewimpertem hiel und zwei weniger behaarten Randnerven, die schmälere, fast gleich lange Vorspelze umgreifend. Blüte August bis Oktober.

Auf Schutt bei Hamburg selten.

Hamburg: bei der Wollkämmerei am Reiherstieg (Kansch 1895!. J. Schmidt 1897!), in einer Kiesgrube in Bahrenfeld 1905̃!!.

\section{Formen.}

f. glabra nov, f. - Blätter bis $13 \mathrm{~cm}$ lang, schlaff, dunkelgrün, völlig kahl, am Rande nicht oder nur gegen die Spitze schwach rauh. Deckspelze schwach behaart.

Hamburg: Bahrenfeld, in dichtem Bestande höherer Pflanzen!!.

\section{Gattung.}

\section{Chloris.}

Swartz Fl. Ind. Occ. I. 189 (1797).

1. Deckspelze am oberen Ende behaart................. barbata.

- Deckspelze am oberen Ende kahl .......................... 2

2. Deckspelze zwischen zwei kurzen Seitenspitzen begrannt....... . truncata.

- Deckspelze zwischen zwei haarartig ausgezogenen Seitenspitzen lang begrannt.

C. radiata.

26. (adv. 17). Chloris barbata swartz Fl. Ind. Occ. I. 200 (1797).

$\odot$. Stengel liegend oder aufsteigend, $0,3-0,5 \mathrm{~m}$ hoch, glatt, oft verzweigt. Blattscheiden etwas rauh, am Rande öfter behaart. Blattfläche 3-4 mm breit, flach, rauh. Blatthäutchen fast fehlend. Rispe mit $4-8$ fingerig gestellten, $4-6 \mathrm{~cm}$ langen Älıren. Ährchen 3,5-4 mm lang. Untere Hüllspelze liurz; obere Hüllspelze doppelt so ling wie die untere, spitz grannenartig ausgezogen. Deckspelze mit $7-8(-10) \mathrm{mm}$ langer (iranne, etwa :3 mm lang, oberwärts mit zalhlreichen, dichtstehenden, bis 1,5) $\mathrm{mm}$ langen, weifen Haaren. Ährchenachse verlängert, mit zwei kurzen, spitzen, leeren Spelzen. Blüte August bis Oktober.

Auf Schutt bei Hamburg selten.

Hamburg: bei der Wollkämmerei am Reiherstieg (Zimpel 1894!). 
27. (adv. 181. Chloris radiata siwat\% Fl. Ind. ()er. I. 20)1 (1797).

$\odot$. Stengel.in der Regel aufrecht, $0,2-0,5 \mathrm{~m}$ hoch, glatt, oft verzweigt. Blattseheiden meistens sehwateh rauh, kahl. Blattfläche $5-6 \mathrm{~mm}$ breit. Hach, sehwarh rauh. Blatthäutehen fisst fehlend. Rispes mit 5-12 (selten mehr) tingerig gestellten. 4-6 am langen Ïhren. Ährohen etwa $3 \mathrm{~mm}$ limg, mit zwej Hü̈lspelzen. Hüllspelzen häntig, gekielt. mgleich lang. Deckspelze mit bis $15 \mathrm{~mm}$ langer Granne, mit zwei seitlichen, harartig ansegegenen spitzen. ohne Hatrsehopt. Blüte Angust bis Oktober.

Auf Schutt bei Hamburg selten.

Hamburg: bei der. Wollkämmerei am Reiherstieg 1.J. Sh.hmidt 1894!. Deutsche Bot. Monatsischr. XT1. 5t [1896]). (Dort als [symonym] C. pullidn Willd. Spec. plant. IV. 926 [1805]).

28. (adtr. 19). Chloris truncata R. Brown Prodr. Fl. Nov. Holland. 186 (1810).

$\odot$. Stengel am Grunde niederliegend, oberwärts aufsteigend, oft wurzehnd. glatt. Blattscheiden glatt. kahl. Blattfliche $2-6 \mathrm{~mm}$ breit, oft gefaltet, glatt. kahl. Blatthäutchen fast fehlend. Rispe in der Regel mit 8-10 fingerig gestellten, bis $8 \mathrm{~cm}$ langen Ähren. Ährchen etwa $4 \mathrm{~mm}$ lang, mit zwei ungleichen. spitzen Hülspelzen. Deckspelze tast 4 mm lang, am Ende mit zwei kurzen spitzen. aus dem Einschnitt begrannt, dreinervig mit randständigen. wimperig beharten seitlichen Nerven. Granne etwa $8 \mathrm{~mm}$ lang, doppelt so lang wie das Ährchen. Ährchenachse über der Blüte verlängert. kihh. mit in der hegel zwei kurzen, $2 \mathrm{~mm}$ langen, breit abgestutzten. begramnten sipelzen. Blïte Angust bis Oktober.

Auf Schutt bei Hamburg selten.

Hamburg: bei der Wollkämmerei am Reiherstieg (.J. Nohmidt 1894!. Laban 1895!. 1896!) (Deutsche Bot. Monatsschr. XIII. 111 [1895] als Chloris barbuta z. 'T., XIV. 54 [1896]).

13. Gattung:

\section{Beckmannia.}

Host Gram. Austr. III. 5 (1805).

29. (adr. 20). Beckmannia eruciformis Host (iram. Anst1. III. j) (1805).

4. Stengel anfrecht, 0,5-1 m hoch, glatt. Blattscheiden lang,

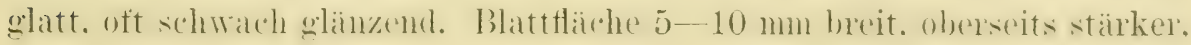


unterseits schwärher rauh. lang gestreckt. Blütenstandsachse langgestreckt. in mgleicher Höhe die seitlich abstelenden. kurzen Ähren tragend. Ähen $1-3$ cm lang. zuweilen mit wenigen grundständigen Zweigen. Ährehen zweiblütig. fast $3 \mathrm{~mm}$ lang und fiast elenso breit. seitlich zusammengedrückt. Hüllspelzen fast grleich. kahntörmig. mit gezähneltem Kiel. Decksuelze gekielt, auf dem Rürken kurz behart. Blüte August und September.

Auf Schutt bei Hamburg selten.

Hamburg: anf Baggererde im Hammerlorook (.J. A. Schmidt 1875!). am Diebsteich in Altona (C. Timm Ber. Deutsche Bot. Ges. V. CIII [1887], Prahl Krit. Fl. 245 [1890], Laban 1885!).

\section{Gattung.}

\section{Eleusine.}

Gaertner Fruct. I. 8 (1788).

1. Ähren gestreckt, etwas locker; Ährchen 4 mm lang .E. indica.

- Ähren kurz; Ährchen dicht gedrängt, 5-6 mm lang E. tristachya.

30. Gadr. 21). Eleusine indica (iitertnel Fruct. I. \& (17-s).

$\odot$. Stengel aufrecht, oft rerzweigt, $0,2-0,5 \mathrm{~m}$ hoch, glatt. Blattscheiden glatt. oberwirts am Rande langharigg gewimpert. Blattläehe 3 - li mm breit. glatt. kahl oder nur dicht vor der scheidemmündung schwarh lang gewimpert. Ähren fingerig gestellt. in der Regel zu $3-6$. zuweilen einzelne an stengel abwïrts gerücht. $4-8$ cm lang. bis 5 mm breit. Ährchen gedrängt, etwa $4 \mathrm{~mm}$ lang, 4-6 blütig. Hüllspelzen etwas ungleirh. 1.5 und $2 \mathrm{~mm}$ lang. gekielt. spitz. Deckipelzen $2.5 \mathrm{~mm}$ lang, spitz, mit rauhem, grünem Kiel. Blüte Angust bis September.

Auf Schutt bei Hamburg selten.

Hamburg: bei den Altonaer Wasserwerken bei Blankenese (Prahl Ber. Deutsche But. Ges. X. s6 [1892]. Zimpel 1891!. .J. schmidt 1892!.

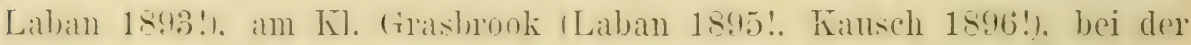
Wollkämmerei am Reiherstieg (J. Schmidt 1899!).

31. (adr. 2.2). Eleusine tristachya Kunth Enumeratio I. 273 (1833).

$\odot$. Stengel meistens aufsteigend, $0,1-0,3 \mathrm{~m}$ hoch, glatt. Blattscheiden glatt. kahl. oberwärts offen. Blattliache 2-4 mm breit. kahl. am Rande schwach rauh. Blatthäutchen fehlend. Ähren zu (1-)2-3, tingerig gestellt wler eine abgerücht. $1-34-5) \mathrm{cm}$ lang. bis $9 \mathrm{~mm}$ breit. 
sehr dicht. Ährehen meistens 5-6 blütig, 5- 6 mm lang. Hüllspelzen mngleich, 1-1,5 und $2 \mathrm{~mm}$ lang. Deckspelzen eifömig-spitzlich, fïnfnervig, grün, kahl, etwa $3 \mathrm{~mm}$ lang. Frucht schwach kantig, fast kugelig. Bliite August bis September.

Auf Schutt bei Hamburg selten.

Hamburg: bei der Wollkammerei am Reiherstieg (.J. Schmidt 1894!), am Worthdamm auf dem Kl. Grasbrook (Zimpel 1896!, 1897!).

Die Pflanze ist zuerst als $E$. coracana $\mathrm{L}$. angegeben worden.

15. Gattung.

\section{Dactylotenium.}

Willdenow Enumeratio II. 1029 (1809).

32. (adv. 23). Dactylotenium aegyptiacum II'illd. Fnum. II. 1029 (1809).

$\odot$. Stengel zu mehreren, oberwärts aufsteigend, 0,05-0,3 m lang; glatt, kahl. Blattscheiden etwas gekielt, breit, die oberen etwas aufgeblasen, alle kahl. Blattflache 3-6 mm breit, glatt, am Rande von auf Knötchen stehenden Haaren lang gewimpert. Blatthäutchen kurz, breit. Ährchen in fingerig zu 5-10 gestellten, 6-8 mm langen Ähren, dicht gedrängt abstehend, $4 \mathrm{~mm}$ lang, zweiblütig. zuweilen mit dem Ansatz einer dritten Blüte. Hüllspelzen etwa 2 mm lang, kahl, eimnervig. Deckspelzen fast $4 \mathrm{~mm}$ lang, kahl, gekielt, eiförmig-spitz, undeutlich nerrig. Vorspelze häutig, kürzer als die Deckspelze. Blüte September.

Auf Schutt bei Hamburg selten.

Hamburg: bei der Wollkämmerei am Reiherstieg (Ascherson und J. Schmidt 1899!) (A. u. Gr. Syn. Mitt.-Europ. Fl. II. 1. 169 [1899]).

16. Gattung.

\section{Milium.}

L. Gen. plant. ed. 5. 30 (1754) z. 'T.

1. Pflanze 4; Rispenäste glatt, absteheud bis zurückgeschlagen; Hüllspelzen glatt.

II. effiusum.

- Pflanze $\odot$; Rispenäste rauh, aufrecht und anliegend; Hüllspelzen rauh.

M. vernale.

33. (sp. 5). Milium effusum L. Spec. plant. ed. 1. 61 (1753).

4. Grundachse kurze Ausläufer treibend. Stengel $(0,4-) 0,6-1 \mathrm{~m}$ hoch. Blattscheiden glatt, kilil. Blattläche $8-10(-15) \mathrm{mm}$ breit, ober- 
und unterseits schwach. am Rande stark rauh. kahl. Blatthäutchen meistens $4-6 \mathrm{~mm}$ lang. zerrisese. Rispe $10-201-301$ (an lang. sehr locker. mit ab-

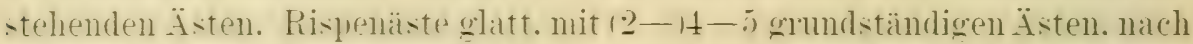
der Blïte oft al,wärts geneigt. Ährohen meistens grün, $3 \mathrm{~mm}$ lang. einblütig. Hïlspelzen fast gleirh. dleinerrig. spitzlich. glatt oder sehr schwach rauh. Deckivelze etwa 2.5) mm lang. dreinervig. gramenlos, die -2 mun lange Frucht und die gleichartige Turvelze in derReife fest mongreifend. Blüte Mai bis. Tuli.

In Wäldern. besonder's lichten Laubwäldern, und Gebüschen im Osten häutic. im Westen nicht selten. Auf den nordtriesischen Inseln nur von siylt und Föhr: hier und darschiotz. 1 thil ] genannt. aber seither nicht wiedergefunden.

Die erste Erwähnung ans dem (rebiete gibt Weber Prim. Fl. Hols.6[17s(1]).

\section{Formen.}

Nicht beobachtet.

34. 1arly. 24). Milium vernale II. v. B. Fl. Taur.-Cauc. I. 53) 11808 ).

E. Grundachse mit in der Recrel mehreren bis zahlreichen Stengeln. stengel 0.2-0.4 m hoch, aufrecht. meistens glatt. nur unter der Rispe schwach rauh. Blattscheiden lang. die oberen zuweilen etwas erweitert. glatt. kahl. Blattfläche $2-t \mathrm{~mm}$ breit. shwach rauh. Blatthäutchen 3-5 mm lang, ungeteilt. Rispe etwas locker, 4-8 cm lang, mit aufrecht abtehenden, meistens geschlängelten Ästen. Rispenäste schwach rauh. mit bis sechs grundständigen Ästen. Älnrchen wenig über $2 \mathrm{~mm}$ lang. einblütig. Hüllspelzen mit starkem Mittehners und zwei schwachen Seitemerren. spitzlich. runzeligr gepunktet. Deckspelze $2 \mathrm{~mm}$ lang. gramenlos. schwach glänzend. Blüte Mai bis .Juni. selten im Herbste.

Auf Schutt bei Hamburg selten.

Hamburg: bei der Wrollkämmerei am Reiherstieg IZinnel 1894!. am 23. September in Blüte!. hei der Wandsheher Dampfmühle (.J. Sichmidt 1843!. 1894!. an der sirrichbrücke in Winterhude (Zimuel 1894!) am Diebsteich in Altona (Fausch 1-85. C. Timm 1865.. 18s6!: vgl. Prahl Krit. Fl. 251 [1890]).

\section{Gattung.}

\section{Stupa.}

L. Gen. plant. ed. 5. 34 (1754).

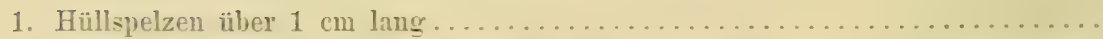

- Vntere Hüllspelze bis $9 \mathrm{~mm}$, obere bis $7 \mathrm{~mm}$ lang. ........ formicarum.

2. Pflanze 24; Deckspelze unterwärts hehaart, oberwärts gekörnelt rauh, aus gezähneltem, kurzem Aufsatz hegramnt .................. intricata.

- Pflanze $\odot ;$ Deckspelze behaart, ohme Aufsatz................. tortilis. 
35. (adv. 25). Stupa tortilis I)efontaines Fl. Atlant. I. 99 (1798).

$\odot$. Stengel zu vielen, aufrecht oder aufsteigend, $(0,1-) 0,3-0,5 \mathrm{~m}$

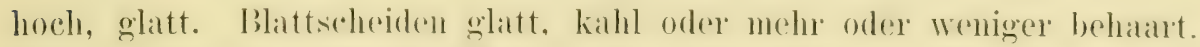
Bläter gratugrün, gofaltot. Blatthäutehen fast fohlend. Rispe gestreckt, mit wenige Ährohen tragenclen Rispenästen. Rispenäste kum, sohwarh

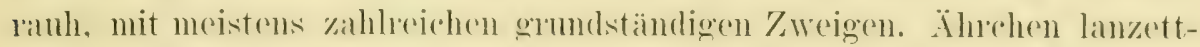
lich, etwa $2 \mathrm{~cm}$ lang. Hüllspelzen ungleich, die untere etwa $2 \mathrm{~cm}$, die obere 1,5 cm lang. bis anf die Mittelriple häutig, spitz. Deckspelze bis über $8 \mathrm{~mm}$ lang, unterwärts dicht weißhaarig, oberwärts zerstreut behaart, mit Granne. Gramme bis $10 \mathrm{~cm}$ lang, unterwäts stark gedreht und behaart, oberwärts stark rauh. Blüte Mai bis Juni.

Auf Schutt bei Hamburg selten.

Hamburg: bei der Wollkämmerei am Reiherstieg (Zimpel 1897!).

36. (adv. 26). Stupa formicarum Delile Ind. Sem. Hort. Momsp) 7 (1879). 4. Stengel zu mehreren, autiecht, 0,5-1 m hoch. Blattscheiden glatt, kahl. Blattflähe gefaltet, kahl. Rispe gestreckt, zusammengezogen, ihre Iste mit zwei bis drei grundständigen / weigen. Ahrchen bis fast $1 \mathrm{~cm}$ lang, lanzettlich. Untere Hüllspelze bis $9 \mathrm{~mm}$, obere bis $7 \mathrm{~mm}$ lang, mit zwei schwächeren Seitemerven. Deckspelze etwa $6 \mathrm{~mm}$ lang, an der Spitze mit gezähneltem, schief abgeschnittenem Aufsatz. der die etwa 4-5 cm lange Granne trägt. Blïte Juni bis Juli.

Auf Schutt bei Hamburg selten.

Hamburg: bei der Wollkämmerei am Reiherstieg (.J. schmidt 1894!, 1896!; vgl. Deutsche Bot. Monatsschr. XTT. 54 [1896] [als S. Mygalina]).

37. (adv. 27). Stupa intricata (iodron Mém. Acarl. Montpellier I. 449 (1858). 4. Stengel zu mehreren, aufrecht, 0,3-0,5 m hoch, glatt. Blattscheiden glatt, kahl. Blattliache gefaltet. Rispe etwas locker, gestreckt. Rispenäste mit $1(-2)$ grundständigen Zweigen. Ährehen bis reichlich $1.5 \mathrm{~cm}$ lang; lanzettlich. Hüllspelzen sehmillanzettlich, spitz ausgezogen, dreinervig, die mutere 15 , die obere $13 \mathrm{~mm}$ lang. I)eckipelze ofwa $8 \mathrm{~mm}$

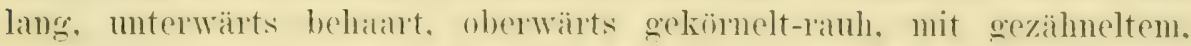

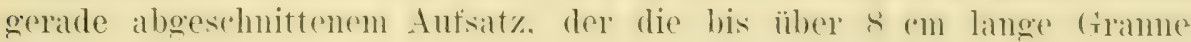
trägt. Blüte bei uns Oktober.

Als Schutt bei Hamburg selten.

Hamburg: bei der Wollkimmerei am Reiherstiog (Zimpel 13. 10. 1895)!) mit S. formicarem.

Stupa pennate L. Spec. plant. ed. 1. 78 (1753) ist mehrfach aus dem Gebiete genannt worden, zuerst für Hamburg durch Buek (Hoppe Bot. Taschenb. 91 [1801]), sodam 
von Nolte (Novit. Fl. Hols. 12 [1826]) ohne Standortsvermerk und nach seiner Aufzählung von Borchmann (Holst. Fl. 72 [1856]). Die zu dieser Art von Knuth (Fl. v. Schlesw.Holst. 756 [1887]) gemachten Ausführungen sind mehr als belanglos; vgl. dazu Prahl (Krit. Fl. 251 [1890]). Wenn an letzterer Stelle darauf hingewiesen wird, dab eine Angabe MöBlers auf die Bueksche Notiz zurückgehen dürfte, so ist demgegenüber zu bemerken, daß Mößler zwar S. pennata aufzühlt (Handb. Gewïchskunde 95 [1815]), Hamburo aber als Ort des Auftretens nicht nennt. Zu S. pennata gehört möglicherweise P. Kyllings "Gramen exile durum majus; Großer Katzenbart" (Viridarium Danicum 45 [1688]) von Hadersleben: Skrydstrup; vgl, von Fischer-Benzon (Schriften Nat. Ver. Schlesw.-Holst. VIII. 14 [1889]). Das Gras scheint früher gelegentlich gepflanzt worden zu sein.

\section{Gattung.}

\section{Nardus.}

L. Gen. plant. ed. 5. 27 (1754).

38. (sp. 6). Nardus stricta L. Spec. plant. ed. 1. 53 (1753).

4. Grundachse dichtrasig, kurz kriechend. Stengel starr aufrecht, $0,1-0,3(-0,6) \mathrm{m}$ hoch, oberwïrts blattlos, unterwärts glatt, oberwärts rauh. Blattscheiden eng, glatt. kahl. Blattfliche in der Regel starr, borstlich eingerollt. Blatthäutchen bis உ mm lang. Ähre lineal, mit zwei Reihen einseitig gestellter. dicht gedrängter Ährchen. Ährchen fast lineal. spitz, einblïtig. Hüllspelze nur ansnahmsweise entwickelt, in der Regel fehlend. Deckspelze bis $8 \mathrm{~mm}$ lang, mit gramenartig ansgezogener, etwa $3 \mathrm{~mm}$ langer spitze, an Kiel und an der Spitze gewimpert, viel länger als ilıre Vorspelze. Blüte Mai bis Juni.

Auf trockenem bis mäbig feuchtem Boden der Heiden, Mroore. sandigen Ufer und ihrer Mischformationen und C̈bergänge durch das mittlere und westliche Gebiet nicht selten, oft in großer Mrenge, anch häufig auf den Tordfriesischen Inseln Föhr. Amrum, Sylt und Röm, im östlichen Gebiet mehr zerstrent und auf Alsen nur bei Meelsfeld.

Die erste Erwähnung ans dem Gebiete gibt Weber (Prim. Fl. Hols. 5 $[1780])$.

Formen.

f. elatior J. Schmidt nov. f. - Stengel $0,5-0,6$ m hoch; Blätter sehr verlängert, etwas schlaff.

Stormarn: auf Moorboden am Glinder Teich (J. Schmidt 1908!). 


\section{Gattung.}

\section{Mibora.}

Adanson Fam. II. 495 (1763).

39. (sp. 7). Mibora minima Desvaux Fl. Anj. 48 (1827).

$\odot$ und $\odot$. Grundachse dichtrasig. Stengel 3-10 $\mathrm{cm}$ hoch (selten höher), dium, glatt, oberwirts blattlos. Blattscheiden glatt. Blattlaiche fein, borstlich eingerollt, kur\%. Blatthäutehen bis ïber 1 mm lang, abgestutzt. Ïhrehen in solmaler Ïhre, bis $1,5 \mathrm{~mm}$ lang. Hülspelzen fast gleieh lang, am Rürken abgerumdet, stumpf, am oberen Ende gezähnelt. Deck- und Torspelze gleich lang, ebenfalls gestutzt mol geröhnelt. kurzbehart, die eiförmige Frucht umschließend.

Auf Sandfeldern im südlichen Gebiet sehr selten und neuerdings nicht beobachtet. Blüte März bis Mai.

Ha mburg: Bergedort (Tolte nach Hornemamn I)ansk ()ee. Plantel. 3. Udg. II. 118 [1837]). Stormarn: zwischen Silk und Friedrichsruh

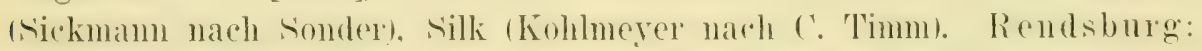
sandiger Heideboden an der Chanssee bei Barlohe bei Hohenwestedt (Hennings 1872!).

Schon Weber nennt diese Art als Agrostis minima aus Holstein (Prim. Fl. Hols. 6 [1780]); Buek gibt sie für Hanburg an (Hoppe Bot. Taschenb. 91 [1801]). Darauf dürfte sich der Vermerk bei Möbler gründen "Hamburg, Holstein" (Handb. Gewächskunde 75 [1815]). Die gleiche Angabe macht Reichenbach (Fl. Germ. excurs. 23 [1830]), der Mößlers Handbuch in zweiter Auflage herausgab. Die erste genauere Bemerkung findet sich bei Hornemann (a. a. 0. 3. Uilg. II. 118 [1837]) für Bergedorf, von Nolte hier beobachtet. Im Kieler und Kopenhagener Herbar ist ein Beleg nicht vorhanden. Sonder erhielt ein Exemplar von Nolte mit "Hamburg" bezeichnet. Homemann hatte $\boldsymbol{M}$. minima schon früher nach Weber und Lehmam (wo gefunden?) aufgenommen (a. a. 0. 3. Udg. I. 73 [1821]), sie auch an anderer Stelle, wieder ohne eingehende Standortsbezeichnung, namhaft gemacht (Vid. Selsk. phys. Skrifter I. Deel. I. Haefte 192 [1821]). Sonder sah nicht nur eine von Tolte gesandte Ptlanze, sondern auch Sickmamnsche Stücke der Art (vgl. Fl. Hamb. 52 [1851]). Da eine Notiz in Sickmanns Enumeratio von 1836 fehlt, muß die Beobachtung nach diesem Jahre liegen. Im $\mathrm{Hb}$. Laban liegen drei Pflanzen der 1T. minima ohne Standortsvermerk, deren Sammler Sickmann sein soll!. C. Timm sah eine Pflanze, welche Kohlmeyer bei silk mitgenommen hatte, und zwar vor 1845. Mit „Silk bei Reinbek" versehen liegt ein Exemplar ohne Finderangabe im Hb. Laban!; es unterscheidet sich von den drei erwïhnten Pflanzen durch die Größe und reiche Stengelentwicklung (etwa 100 Blütenstände). Im Hb. Kohlmeyer (in Lübeck) fehlen Belegstücke. Eine wieder nicht belegte Angabe macht dann noch Borchmann (Fl. v. Holst. 70 [1856]) für das dicht bei Silk gelegene Schönau.

Die Art ist nach 1850 wiederholt in der Gegend ron Silk gesucht, aber nie wiedergefumlen worden. Ihr Indigenat bezweifelt schon Röper (zur Fl. Mecklenb. Teil I. 181/2

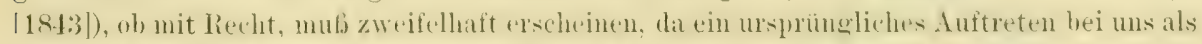
Analogie zum Torknmmen amlerer westlicher Arten wohl möglich ist. Anffallig ist jedenfalls neben der frühzeitigen Erwähnung in der Literatur besonders noch die Erscheinung, daß 
in einer Zeit wie der von 1880-1900, in der zahlreiche Verschleppungen von Pflanzensamen stattfanden, $\boldsymbol{M}$. minima nirgends bei uns auftauchte. Eine Verwilderung aus einem botanischen Garten, wie sie in Kiel stattfand (Jessen Exc.-Fl.), kommt sicher nicht in Frage.

20. Gattung.

\section{Alopecurus.}

L. Gen. plant. ed. 5. 30 (1754).

1. Hüllspelzen am Mittelnerv auf der ganzen Länge oder doch unterwärts zottig

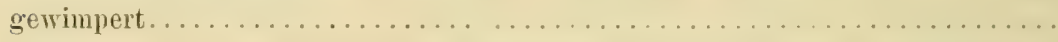

- Hüllspelzen an dem deutlich hervortretenden Kiel mit kurzen Borstenhärchen. A. myosuroides.

2. Hüllspelzen oberwärts plötzlich spitz ausgezogen, unter der Verschmälerung

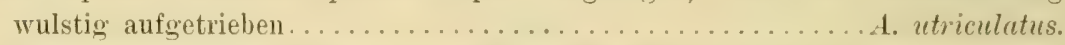

- Hüllspelzen allmählich verschmälert, ohne Wulst................

3. Hüllspelzen nur am Grunde rerbunden, oberwärts mit den Spitzen auseimanderstrebend; Pflanze $\odot$.

- Hüllspelzen im unteren Drittel verbunden, zusammenneigend; Pflanze 4.

4. Deckspelze im unteren Drittel begrannt ................ geniculatus.

- Deckspelze über der Mitte begrannt..................... fulvus.

40. (adr. 28). Alopecurus utriculatus Personon Sym. I. $80(1805)$.

๑. Rasig. Stengel $0,1-0,3 \mathrm{~m}$ hoch, aufsteigend, ziemlich schlaff, glatt, kahl. Untere Blattscheirlen eng, obere erweitert, bis $4 \mathrm{~mm}$ breit, alle kahl, glatt. Blattfläche 2- + mm breit, beiderseits schwach behaart bis kahl. Blatthäutchen fast fehlend. Rispe $1-2 \mathrm{~cm}$ lang, eiförmig bis länglich-eiförmig, dicht. Ährehen einblütig. etwa $6 \mathrm{~mm}$ lang. Hüllspelzen gleich. 5 mm lang. unterwärts bis zur Mitte verbunden, stark gekielt, in der Nähe des Kiels stark behaart, oberwärts verschmälert, spitz. Deckspelze lanzettlich. etwa $6 \mathrm{~mm}$ lang. ihre Ründer am Grunde verbunden, fünfnervig. aus dem Grunde des Rückens mit bis $10 \mathrm{~mm}$ langer Granne. Vorspelze nicht vorhanden. Blüte Mai bis Juni.

Auf Schutt bei Hamburg selten.

Hamburg: bei der Wandsbeker Dampfmühle (.T. Schmidt und Zimpel 1894!), am Diebsteich in Altona (C. Timm 1885!. Laban Fl. r. Hamb. usw. 4. Aufl. 193 [1887] als A. utriculus).

41. (9s). 6). Alopecurus myosuroides Hudson Fl. Angl. ed. 1. 23 (1762). C-mnd $\odot ?)$. Rasig. Stengel anfrecht oder anfsteigend, $0,2-0,5(-0,8) \mathrm{m}$ hoch, schwach rauh. kahl. Blattscheiden glatt oder schwach rauh, die oberen wenig erweitert. Blattliache meistens $3-6 \mathrm{~mm}$ breit, oberseits 


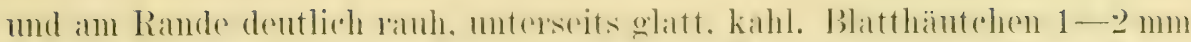

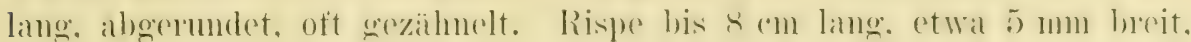

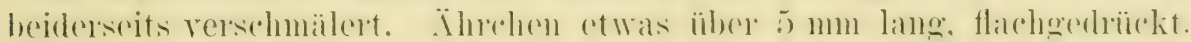

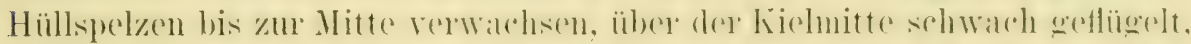

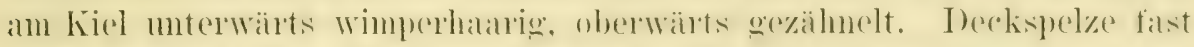

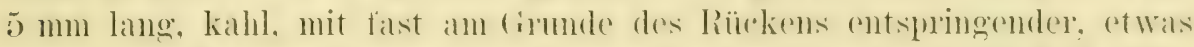
unter der Nitte geknieter, bis $11 \mathrm{~mm}$ langer Gramne. Blüte Mai bis Juni (Juli) und vereinzelt Angust bis Oktober.

Auf Äckern unter der Saat (Raps, Wintergetreide) sowie auf Schntt und an Wegen durch das ganze Gebiet, stellenweise reichlich, doch vielfach unbeständig; im mittleren Gebiet selten.

Lauenburg (Nolte, Hb. Kopenhagen!): Lauenburg (Claudius), Zieten bei Ratzeburg (Reinke 1869 nateh Friedriehs. If a mburg: am Eilbufer dem

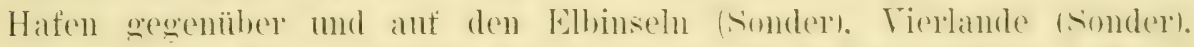
(ieorgwwirder (Zimpel 1893!). Kl. Grasbrook t.I. A. Sehmidt 1871!) steinwärder (Bertram, A. Junge 1896!), Kuhwärder (C. Timm 1868), bei der Wollkimmerei am Reiherstieg (Zimpel 1s!! t!)!. Hammerhrook 1.J. A. Sehmidt 1866!). Hamm (Katusch!). St. Pauli (C. Timm 1867). Thlenhorst (C. Timm!. Winterhude (J. A. schmidt 1869 !). Espendorf (Erichsen 18.4!!). Fuhlsbüttel 1912!!. Stormarn: Wandsbek (Zimpel 1893!), Steilshop 1912!!, Poppenbuittel (A. Mohr 1903!). Pinneberg: am Diebsteich (W. Hansen 1885!) und bei der Holstenbranerei in Altona Zimpel 1s!2!!). Bahrenfeld (Erichsen 1sxa!)!!. Othmarschen (Suse nach Laban), Elottbek (sonder. Deecke!). Nienstedten (Kohlmeyer!), Blankenese (Sonder). Langenfelde (Laban 1893!). Lokstedt (Lab)an 1891), bei der Hetlinger sohanze (Eschenburg 1893!). Lübeck: zwischen Burg- und Hüxtertor (K. Burmester 1!12!). Nenstadt mehrach (Rohweder!). Oldenhurg i. H.: (irube (Nolte 182.2!). Heiligenhafen (Nolte. Hb. Kopenhagen!, I. A. Schmidt 185t!). (irofenbrode (Nolte 1822!) mehrfach!!. Fehmarn (Hemings. ('auben 1894!) rerbreitet!!. Lütjenburg (Borchmann nach Knuth). Kiel verbreitet. Rendsburg: an der Kieler Chanssee (Dreßler, Hb. Prahl!). Angeln: Seegaard bei Markerup) (Hansen!). Sonderbure (sehiotz): Tandslet (Petit). Glüekstadt: Engelbrechtsche Tildnis!!. I)ithmarsehen: st. Michatelisdom (A. Mohr 1901!), Wesselburen (J. J. Meyer 1821!). Eiderstedt: an Deichen (Bargum 1801!) und unter Winterkorn (Hornemanm). Tümning (F. r. Mü̈ller), (hristian-Albrechtskong (Hansen nach Kinuth). Husum: schanenthal (F. r. Mäller). Fresendelf (Hansen nach Finuth). Tondern: Deezhuill (Jörgensen 1835!). Föhr (Hb). Arfsten nach v. Fischer-Benzon). Amrum: Wittlün (Kutkek und Polgar 1906!). Helgoland (Brody!).

Die erste Errwähnung aus den Herzogtïmern gab Weber (Prim. Fl. Hols, $6[1780]$, unter dem bis vor kurzem allgemein gebräuchlichen Xamen A. agrestis L. Spec. plant. ed. 2. 89 (1762). 
Hornemann gibt (Dansk Oec. Plantel. 3. Udg. 1. 170 [1821]) die Art als in den

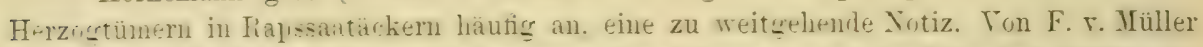

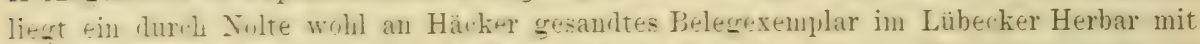
don Vernerk ron Nultes Hand: .. Müller in Frisia “.. Eine genauere Fumhrtshezeichnumg"

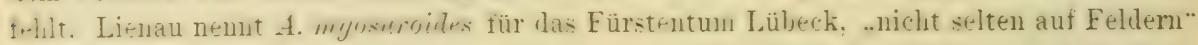
(p. 7 [1863]); Knuth hat Lienau als Gewälırsmann für zwei Standorte: zwischen Eutin und Plön und bei Neukirchen. Die Angaben mögen richtig sein; belegt sind sie nicht, so dab eine Nachprüfung unmöglich ist.

\section{Formen.}

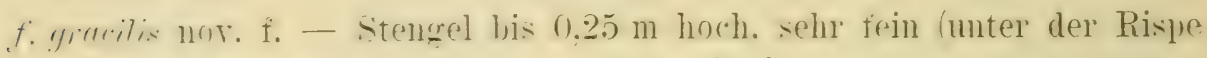
nur $0,3 \mathrm{~mm}$ stark); Blätter $1-2 \mathrm{~mm}$ breit.

Hamburg: bei der Holstenbrauerei in Altona (Zimpel 1891!).

42. 1.1. S) Alopecurus pratensis L. Muec. 1)lant. ed. 1. 1)(1) (153).

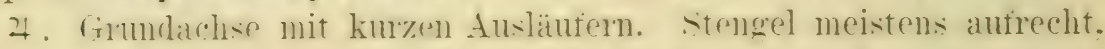

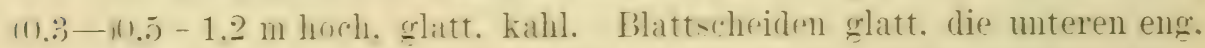
die oberen etwas elweitert. Blattläche $(2-) 4-7(-10) \mathrm{mm}$ breit, oberwits stälker. untrits sohwärher rauh. Blatthäutchen mejstens $2-4 \mathrm{~mm}$

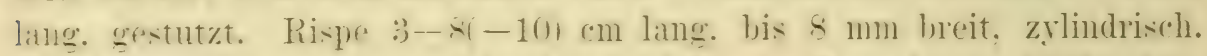

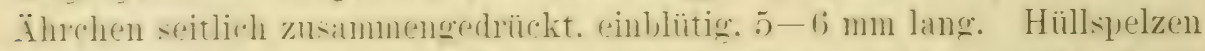

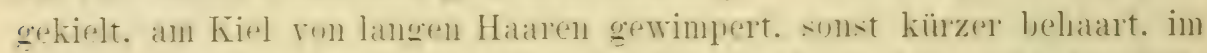

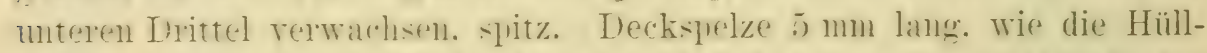

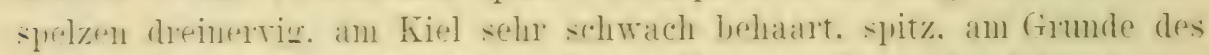

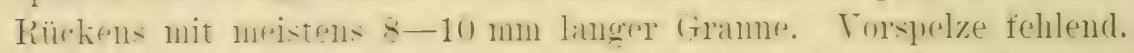

Auf feuchten, seltener trockenen, Wiesen durch das ganze Gebiet,

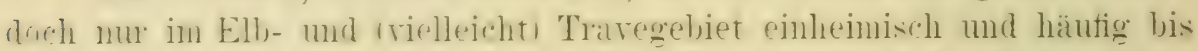

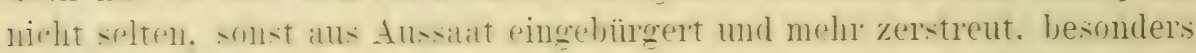

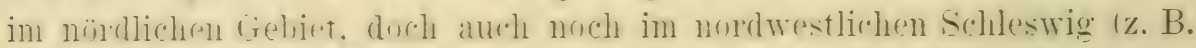

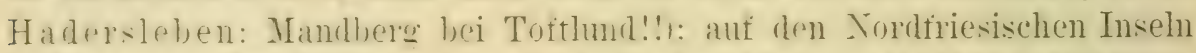
bi-her nur auf sylt: zwischen Westerland und Timmm!!. Föhr: Boldixum

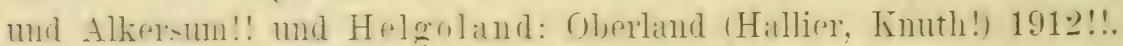

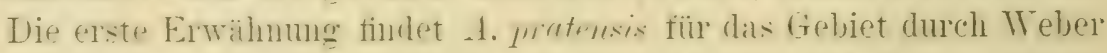
(Prim. Fl. Hols. Suppl. 2 [1787]) von Pinneberg und Kiel: Ziegel-

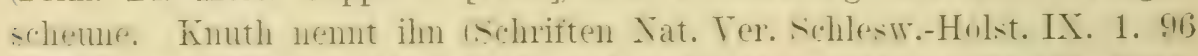

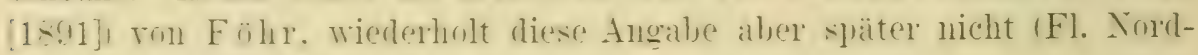
fries. Inseln [1895]), so daß ein Irrtum zu rermuten ist.

\section{Formen.}

1. Pflanze lebhaft grïn; Stengel aufrecht............... 2

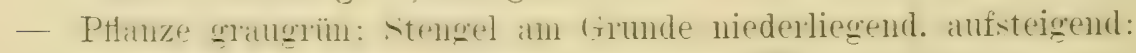
f. glaucus Sonder Flora Hamburgensis 32 (1851). 
Nur im südöstlichen Gebiet. - Hamburg: in den Vierlanden und auf den Elbinseln (Sonder), Geesthacht 1853 und Wilhelmsburg 1869 (C. Timm); von Lauenburg abwärts zerstrent!!. Stormarn: an der Trave bei Oldesloe (Erichsen 1892!).

\section{Dazu:}

f. ascendens Beckmann Abl. Nat. Ver. Bremen X. 510 (1889). - Ährenrispe schmal, oberwärts (oder beiderseits) verschmälert.

Hamburg: Geesthacht (Nolte 1824!), zwischen Bergedorf und Rotenhaus!!.

2. Stengel bis 1,2 m hoch; Rispe bis $10 \mathrm{~cm}$ lang, grün:

f. commun Beck Fl. v. Niederösterreich I. 56 (1890). Häufigste Form.

\section{Dazu:}

f. brachyglossus Petermann Flora XXVII. 232 (1844). Blatthäntchen sehr kurz (bis $1 \mathrm{~mm}$ lang).

Lauenburg: Besenthaler Wiesen bei der Ziehnburger Schleuse (J. A. Schmidt 1868!). Stormarn: zwischen Rethwischholz und Oldesloe!!.

- Stengel meistens $0,4-0.6 \mathrm{~m}$ hoch; Rispe $4-5 \mathrm{~cm}$ lang, dick, dicht, schwärzlich überlaufen:

f. obscums Grisebach in Ledeb. Fl. Ross. IV. 465 (1853).

Längs der Elbe nicht selten (Sickmann, Enumeratio 7 [1836]; Sonder Fl. Hamb. 32 [185̃1])!!; an der 'Trave bei Lübeck!!. - Ubergangsformen verbreitet, z.B. schon Lübeck: Schwartan (Nolte 1821!).

\section{Mißbildungen.}

f. m. vivipara Kuntze Taschenfl. Leipzig 49 (1847). - Ährchen in Laubsprosse auswachsend.

Hamburg: Bergedorf (J. A. Schmidt 1866!). Pinneberg: Holstenbranerei bei Altona (Laban 1892!).

Alopecurus ventricosus Persoon Syn. I. 80 (1805) erw. nennt Röper (zur Fl. Mecklenb. 175 [1843]) aus dem Gebiete unter dem Synonym A. nigricans Hornemamn, auf welcher Grundlage, ist nicht ersichtlich. Schon früher findet sich bei L. Reichenbach der Vermerk: ... . durch das ganze ... mördliche Gebiet" (Fl. Germ. excurs. I. 31 [1830]), eine Angabe, die auch noch in anderen Werken wiederkehrt. Koch nennt als Fundort: „um Hamburg" (Syn. Fl. Germ. et Helv. ed. 2. II. 896 [184t]), vielleicht auf Grund irrtümlicher Bestimmung Sonderscher Exemplare vou A. pratensis $f$. obscums. Was unter A. nigricans "L." bei Hübener (Fl. v. Hamb. 491 [1847]) zu verstehen ist, ist nicht festzustellen, da Exemplare fehlen. Vielleicht ist das Gras an unserer Ostseeküste noch nachzuweisen. 
43. 1.11. 91. Alopecurus geniculatus L. siec. 11:111t. erl. 1. 60 (1753).

() und (?) 4. Stengel zu mehreren, am Grunde niederliegend, oberwärts antsteigend. (0.1-1).5 m lang. glatt. Blattrcheiden glatt, die oberen orlen alle shwach blasig erwertert. Blattfläche $11-1: 3-6 \mathrm{~mm}$ breit. flach

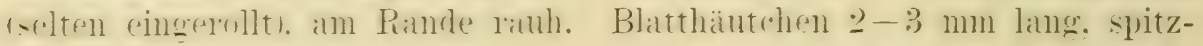
lich. Rispe (1-)3-6 cm lang, (2-) $4-5 \mathrm{~mm}$ dick, dicht, nach oben allmählich shmäles. Ährehen 2.5) mm langr. Hüll-pelzen greich lang; dreinervig. am Kiel wimperharig. sonst zerstrent hehatrt. spitz, oben ans-

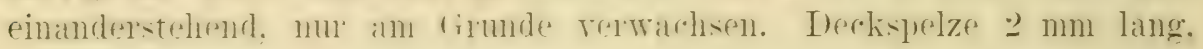
spitzlich, schwach behaart, im unteren Drittel mit $3 \mathrm{~mm}$ langer, das

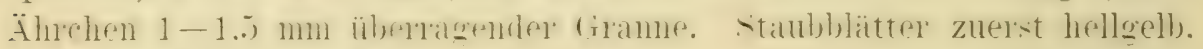
dann braun. $1,5 \mathrm{~mm}$ lang.

An Ufern, an und in Gräben, an quelligen Orten und auf feuchten

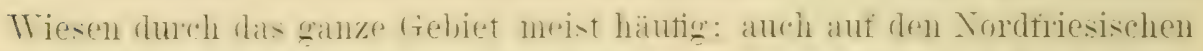
Inseln Nordstrand, Pellworm, Föhr, Amrum, Sylt und Röm sowie auf Helgoland: Oberland (Hallier, Brody!).

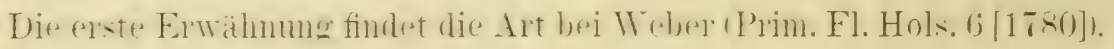

\section{Formen.}

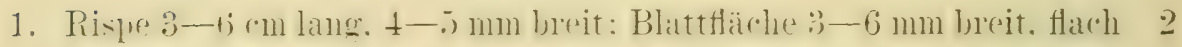

- Rispe 1-2 cm lang, 2-3 mm breit; Blattfläche $1-2 \mathrm{~mm}$ breit, borstlich gefaltet:

var. microstachys Uechtritz in Fiek Fl. v. Schlesien 500 (1881). Rasig, mit zahlreichen nicht blühenden Sprossen und wenigen Stengeln; Stengel sehr fein; Blattläutchen bis $1 \mathrm{~mm}$ lang; Rispe schwärzlich überlaufen. - Eine durch den Standort auf trockensandigem Boden wie durch die gefalteten Blätter, die kurze, schmale Rispe, die kleinen Ährchen und den ITuchs sehr auffällige Form.

Selten. - Sylt: zwischen Keitum und Archsum 1912!!.

2. Stengel aus niederliegendem Grunde aufsteigend .......... 3

- Stengel schlaff, in seichtem Wasser flutend:

f. nutans Wahlenberg Fl. Lapp. 22 (1812).

An ïberfluteten Orten zerstrent.

3. Stengel am Grunde an den Knoten nicht verdickt.......... 4

- Stengel am Grunde an den Knoten knollig rerdickt:

f. bulbases Sonder Fl. Hamb. 32 (1851).

Selten. - Stormarn: Steinbek; Pinneberg: Blankenese (Sonder). — Im Kieler Herbar Nolte 1862!), Standort unleserlich. 
4. Stengel an den Knoten nicht wurzelnd: häufigste Form.

- Stengel an den Knoten wurzelnd, Aste treibend:

f. radicans P. Junge Jahrb. Hamb. Wiss. Anst. XXII. 61 (1904).

'Zerstreut; z. B. Hamburg: Bahrenfeld!!. Segeberg:

Krems!!. Preetz: Schellhorn (A. Christiansen!).

44. (sp). 10). Alopecurus fulvus smith Engl. But. XXI. t. $14 ! 7$ (1405)).

$\odot$. Stengel zu mehreren, aus niederliegendem Grunde aufsteigend oder schräg aufrecht, $0,2-0,4(-0,5) \mathrm{m}$ lang, einfach oder aus den oberen Knoten verzweigt, glatt oder dicht unter der Rispe schwach rauh. Blattscheiden der oberen Blätter etwas erweitert, in der Regel glatt. Blattfläche 2-4 mm breit, an Rande rauh. Blatthäutchen bis $5 \mathrm{~mm}$ lang;

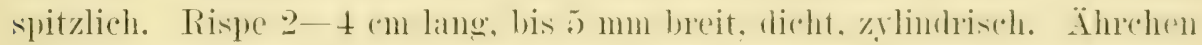
(1.5--)2 num lang. Hüllspelzen gleich lang. nut an filumbe verwachsen. am Kiel lang gewimpert, sonst kuzharig. Deckspelze breiter als die Hüllspelzen, dreinervig, efwat atus der Rülekemmitte mit $11-1.5)$ mm langere. das Ähehen nicht-oder wenig überragender (riane. Ntaubblatter 1 mm lang, rotgelb. Blüte Juni bis Oktober.

An Ufern, in Gräben, in Wasserlöchern und auf nassen Wiesen im sïdöstlichen Gebict meistens nicht selten, nordwäts an Häufigkeit abnehmend bis ins südliche schleswig. Siehleswis: Lü̈schan 1826! und sïderholzkrug 1851! (L. Hansen). Angeln: am Tolker see (Himrichsen 1852)!), an Tolkwader see (Aander $186 t$ in Terhandl. Bot. Ver. Brand. TI. 231 [1864]; dort entstellt, Tolkwar”). Langhalligan (Hb. Hinrichsen!). Husum: Südermarsch (Hansen). A penrade (Sander a. a. O.). - Bei Hamburg auch als Adventivpflanze beobachtet.

Die erste Erwähnung aus dem Gebiete gibt Hornemann (Flora Danica t. 1804 [1825]) aus Lauenburg; wo Nolte A. fulvus aufgefunden hatte (Nov. Fl. Hols. $27[1826])$. I ats äleste von mir gesehene Exemplar sammelte J. J. Neyer 1814 bei Hamburg!.

Formen.

f. natans Groß Allg. Bot. Zeitschr. VIII. 30 (1902). - Stengel flutend, meistens reich verzweigt.

Selten.

$\times(\mathrm{s}) .8 \times 9)$. Alopecurus pratensis $\times$ geniculatus $=$ A. hybridus Wimmer Fl. Schles. 3. Aufl. 31 (1857).

4. Stengel zu mehreren, am Grunde niederliegend, oberwärts aufsteigend, seltener rom cirunde antsteigend, dium. glatt. Blattscheiden im 
oberen Teile des Stengels aufgetrieben. Blattfläche meistens $3-6 \mathrm{~mm}$ breit, oberseits rauh, unterseits glatt. Blatthäutchen 2-5 mm lang, gestutzt. Rispe $3-5(-8) \mathrm{cm}$ lang, etwa $6 \mathrm{~mm}$ breit. Ährchen $4-4,5 \mathrm{~mm}$ lang. Hüllspelzen gleich, im unteren Viertel verbunden, oberwärts nicht genähert, am Kiel lang gewimpert, sonst kurzhaarig. Deckspelze 4 mm lang, stumpf, nahe am Grunde begramnt. Gramne bis $8 \mathrm{~mm}$ lang. Blüte Juni bis Angust.

Selten. - Helgoland: Oberland nicht weit von der großen Sapskuhle dicht am Flagenberg (Hallier Bot. Zeitung XXI. Beilage zu Ni. 19,20). $6 / 7[1863])$.

Von mir im Gebiet vergeblich gesucht, aber südlich der Elbe beobachtet.

\section{Gattung.}

\section{Phleum.}

L. Gen. plant. ed. 5. 29 (1754).

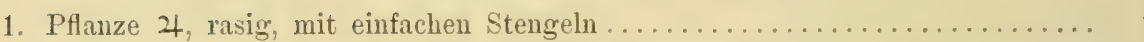

- Pflanze $\odot$ oder $\odot$, einstengelig oder büschelig-mehrstengelig; Stengel unter-

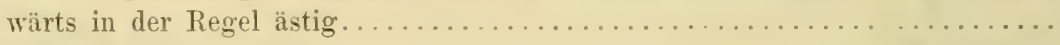

2. Hüllspelzenkiel gerade......................... pratense.

- Hüllspelzenkiel auswärts gewölbt................... Boehmeri.

3. Deckspelze fünfnervig ....................... subulatum.

-- Deckspelze dreinervig.............................. 4

4. Hüllspelzen rauh, etwa $2 \mathrm{~mm}$ lang ................ paniculatum.

- Hüllspelzen am Kiel gewimpert, etwa $3 \mathrm{~mm} \operatorname{lang} \ldots \ldots \ldots \ldots \ldots \ldots \ldots \ldots \ldots$

5. Rispe kurz, dicht.............................. arenarium.

- Rispe verlängert, lappig....................... graecum.

45. (s1). 11). Phleam pratense L. Spec. plant. ed. 1. 159 (1653).

4. Rasig, kurz ausläufertreibend. Stengel meistens aufrecht, 0,2-1,5 m hoch, glatt. Blattscheiden glatt, die oberen sehr wenig erweitert. Blattfläche $(2-) 4-b(-10) \mathrm{mm}$ breit, oberseits stärker, unterseits schwächer rauh. Blatthäutchen 2-6 mm lang. stmmpf bis spitz. Rispe zylindrisch, $(1-13-81-20) \mathrm{cm}$ lang. bis $8 \mathrm{~mm}$ breit, dicht. selten etwas locker. Ährchen 3-5 mm lang. seitlich zusammengedrückt, einl, lütig. Hüllspelzen gleich lang, gekielt, am hiel wimperhaarig, sonst kurzhamig. dreinerrig. plötzlich in die etwa 1 mm lange gramenartige Spitze verschmälert. Deckspelze 2-2,5 mm lang. mit drei stärkeren und zwei schwächeren seitlichen Nerven, häutig. Torspelze schmal. zweinervig, so lang wie die Deckspelze. Blüte Juli bis August, einzeln später.

Auf Wiesen und Weiden, an Abhängen, an Wegrändern und in Wäldern durch das Gebiet häufig, auch auf den Nordseeinseln meist häufig (selbst auf Helgoland!!), doch auf Röm bisher nur bei Kirkeby (.Taap). 
Die Art wird zuerst ron Weber genannt (Prim. Fl. Hols. 6 [1780], anch var. nodosum.

\section{Formen.}

1. var. typicum Beck Fl. v. Niederösterreich I. 55 (1890). — Pflanze

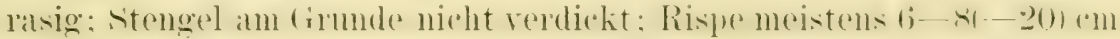
lang; Ährchen $5 \mathrm{~mm}$ lang.

Häufigste Form, besonders auf fruchtbarem Boden.

- var. nodosum Schreber Grïser I. 102 (1769). - Ptlanze rasig bis lockerwüchsig: Stengel am tirunde knollig rerdickt: Rispe 1-4-birm lang; Ährchen etwa $3 \mathrm{~mm}$ lang.

So weniger häufig; doch nicht selten und stellenweise, besonders auf trockenem, sandigem Boden die vorherrschende Rasse, so z. B. auf den Nordseeinseln.

Dazu:

f. abbreviatum Boissier Voyag. II. 633 (18t5). - Stengel etwa $1 \mathrm{~mm}$ stark, $0,1-0,3 \mathrm{~m}$ hoch; Blätter schmal, oft zusammengefaltet; Rispe rundlich oder eiförmig.

Typisch selten. - Föhr: Nieblum!!. Sylt mehrfach. Ubergangsformen finden sich zerstreut.

f. laxiusculum A. u. Gr. Syn. Mitt.-Europ. Fl. II. 1. 143 (1898)。 Stengel kräftiger, $0,3-0,4 \mathrm{~m}$ hoch; Blätter flach; Rispe länglich, zylindrisch, 2,5-4(-6) $\mathrm{cm}$ lang.

Häufigste Form der Rasse.

f. Wamstorfii A. u. Gr. a. a. O. II. 1. 143 (1898), - Stengel niederliegend, nur im obersten Teile aufgerichtet.

Pinneberg: am Elbufer in Othmarschen (Laban, $\mathrm{Hb}$. W. Hansen!). Stormarn: Reinfeld!!. Kiel und Husum: Oster-Ohrstedt (A. Christiansen!). - Als Ubergangsform scheint f. stoloniferum Knuth (Fl. Nordfries. Inseln 142 [1895]) mit an den unteren Knoten wurzelndem Stengel hierher zu gehören.

\section{MiBbildungen.}

f. m. bracteatum A. Braun in A. u. Gr. a. a. O. II. 1. 142 (1898). Rispe am Grunde mit in Scheide (weit geöffnet) und Spreite zerfallendem Laubblatte.

Stormarn: Wandsbek (J. A. Schmidt 1867!). Stormarn: Gr. Barnitz und Frauenholz!!. Von Kiel bis Flensburg mehrfach (A. Christiansen!). 
f. m. viviparum nov. f. - Ährchen in Laubsprosse auswachsend.

Hamburg: zwischen Bergedorf und Rotenhaus (J. A. Schmidt 18(55)!. Hammerbrook (Laban!!). Sternschanze (C. Timm), Wollkämmerei am Reiherstieg (Zimpel!). Langenfelde (.J. Sichmidt!). Stormarn: Wandsbek (J. A. Schmidt!). Lübeck: Ivendorf (Häcker)!). Flensburg: Ekensund (Prahl! bei f. nodosum).

f. $m$. furcutum nov. f. - Rispenachse an der Spitze gespalten. Rispe zweiteilig:

Ha mburg: bei der Wollkämmerei an Reiherstieg (Laban 18.66!).

46. (adv. 29). Phleum Boehmeri Wibel Prim. Fl. Werth. 125 (17991.

4. Pflanze mit Laubtrieben und Stengeln. Stengel $0,4-0,6(-0,9) \mathrm{m}$ hoch, aufrecht oder aufsteigend. dümn. glatt. Blattscheiden glatt. zuweilen sehr wentg rauh. die oheren etwas prweitert. Blattfäche $2-4 \mathrm{~mm}$ breit, in der Regel flach, beiderseits und am Rande rauh. Blatthäutchen $1-2 \mathrm{~mm}$ lang. gestutzt. Rispe $(2-50-10(-15) \mathrm{cm}$ lang. gelappt, oben und unten rersehmälert. Ährchen bis wenig über $2 \mathrm{~mm}$ lang: mit über die Blüte rerlängerter Achse. Hüllspelzen fast gleich. spitz, mit gebogenem, spärlich gewimpertem bis rauhem Kiel. Deck- und Torspelze 1.5) mm lang. erstere dreinerrig. zerstrent kurzhaarig. stumptlich. Blüte Juni bis August.

Auf Schutt und an Wegen selten und unbeständig.

Hamburg: am Diebsteich in Altona (Laban und C. Timm: IT. Hansen 1885!). Pinneberg: Nienstedten (M̈̈ßler Handb. Gewächsk. 8t [1815]; sionder; Deecke. Hb. Lü̈beck!). um Nienstedten (Miößler; Sonder 1837!. Hb. Kiel!: J. J. M[erer 1838!) (seit Jahrzehnten an beiden stellen nicht mehr\% Kiel: um Kiel (H. Ackermann. Hb. Kiel!). Schünberg (Hb. J. J. Мeyer 1845!) (ebenfalls seit langem nicht mehr gefunden).

Die erste Angabe der Pflanze aus dem Gebiete bringt Weber (Prim. Fl. Hols. 5 [1780]) ohne Standort. Hübener nennt sie (Fl. v. Hamb. 491/2 [1847]): zwischen Lurup urd Schenefeld; Belege fehlen. Aus Lauenburg hat Klatt (Fl. r. Lauenburg 163 [1865]) den Standort Grönau, als Gewährsmann Luther. Auch diese Angabe ist unsicher. Hallier sagt (FI. v. Helgoland 7 [1863]): „auf einem Acker unweit des Leuchtturms“, wo die Pflanze später nicht wiedergefunden worden ist. Sicher falsch sind die Fundorte: Barmbeck (Nolte) und Niendorf bei Ratzeburg (Nolte) bei Knuth (Fl. r. Schlesw.-Holst. 750 [1887]); es handelt sich um Formen ron P. pratense.

47. (adr. 30). Phleum graecum Boisciel n. Heldreich Diagn. I. XIII. 42 (1853).

$\odot$, $\odot$. Stengel zu mehreren, aufrecht oder aufsteigend, zureilen am cirme verzweigt. 0.15-0,t m hoch. glatt. Blattscheiden glatt oder 
schwach rauh, die oberen etwas erweitert. Blattflache kurz, 2-4 mm

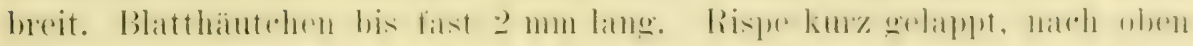
allmählich verschmälert, $3-6(-16)$ cm lang, zuweilen unterbrochen. Ährehen etwas über 3 mm lang. Hüllspelzen dreinerrig, am Kiel und

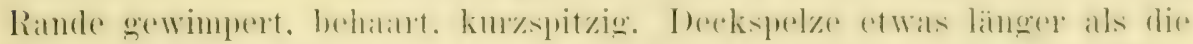

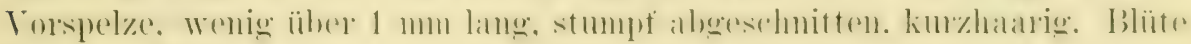
Mai bis Juli.

Auf Schutt bei Hamburg selten.

Hamburg: Georgswärder (Zimpel 1893!, 1894!), bei der Dampfmühle Wandsbek (W. Timm 1892!, Zimpel 1893!, 1894!, J. Schmidt

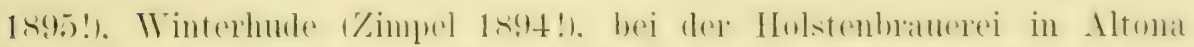

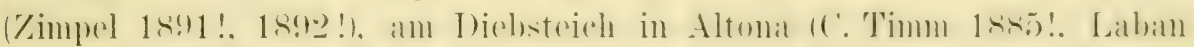
1886!, Erichsen 1887!) (Prahl Krit. Fl. 247 [1890]).

\section{Formen.}

f. Tobatum nov. f. - Rispe locker, im unteren Teile mterbrochen, stark lappig, bis $16 \mathrm{~cm}$ lang:

Hamburg: bei der Wandsbeker Dampfmühle (Zimpel!, Kausch!).

\section{MiBbildungen.}

f. m. furcatum nov. f. - Rispenachse oberwärts zweiteilig.

H a mburg: Dampfimühle Wandsbek (Zimpel 1894!).

48. (sp. 12). Phleum arenarium L. sipece plint. ed. 1. 60 (1753).

$\odot$. Stengel einzeln oder zu mehreren $(-16)$, aufrecht oder aufsteigend. 3-25 cm hoch, olatt. oft wie die scheiden purpur-riolett überlanfen. Blattscheiden schwach bis ziemlich stark erweitert. glatt. Blattfläche 2-4 mm breit, meistens kürzer als die Scheide, oberseits raulh, unterseits glatt. Rispe kurz ghlindrisch. 1-3 cm lang. dieht. Ährehen 3) mm limg. Hüllopelzen allmählich rerschmälert. dreinervig. spitz. wher-

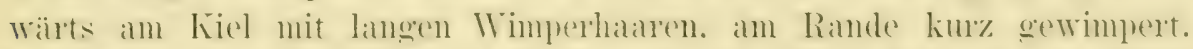

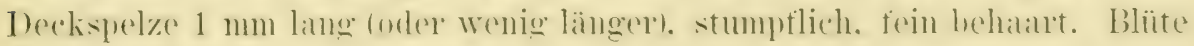
Jai und Juni.

Auf Sandboden an der Ostsee ron Travemünde bis Kiel zerstrent,

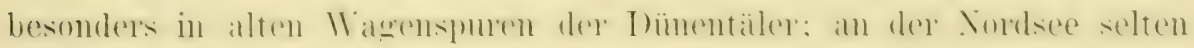
auf Föhr und Röm.

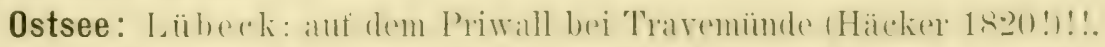

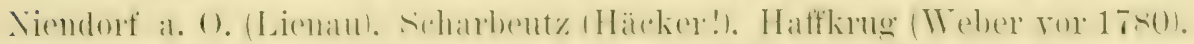
Neustadt: Pelzerwiese (Lienau, Rohweder)!!, Rettin 1823! und Brodan

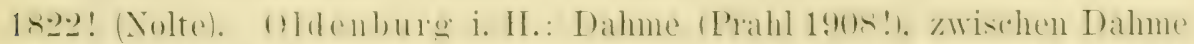

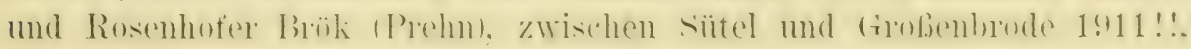




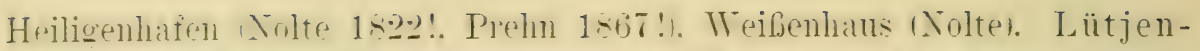
h) nrg: an sehlendonfer Binnenser bei Hohwacht (Erichsen 1895!n!!. .. Liple" bei Habhere 1912!.. Kiel: schönlererer strand (Liban ron 1866. A. Christiansen 1909!), Strande (A. Christiansen 1912!).

Nordsee: Fïhr: (schiötz: Hl). Artsten nach r. Fischer-Benzons. Röm: (Nolte 1*25!): in den Dünen bei Harneby (Prahl 18T4!. J. Schmidt!). west-

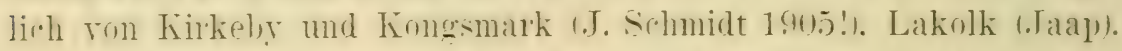

Genannt schon ron Teber für Haffkrug (Prim. Fl. Hols. 6 [1780]).

Die Angabe ron Föhr nehme ich auf, da r. Fischer-Benzon die Pflanze aus dem Hb. Arfsten gesehen hat (Schriften Nat. Ver. Schlesw.-Holst. II. 113 [1876]). Sehr unsicher resp. unrichtig findet sich das Gras rermerkt aus Lauenburg: Gr. Sarau (Luther nach Klatt, Fl. . Lauenb. $16 \pm$ [1865]) und ron der Elbmündung: ,findet sich rereinzelt am Elbstrande, an der Mündung der Stölı̈ (Hübener, Fl. r. Hamb. 492 [1847]).

49. Cardr. 31). Phleum paniculatum Hudson Fl. Angl, 2:3 (1762).

¿. stengel zu melureren. anfstrigend oder ans niederliegendem firunde aufstrigend. nft rerzweigt. clatt. Entere Blattscheiden eng. obere erwritert. in der Regel glitt. Blattlärhe am Rimule rauh. oberseits glatt oder schwach rauh. 3-10 mm breit. Blatthäutchen 1-4 mm lang, zu-

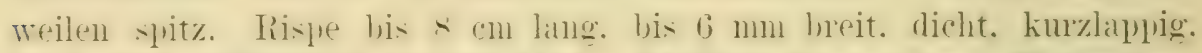
nach oben almählich rershmälert. Älnchen $ّ$ mm lang. Hüllspelzen

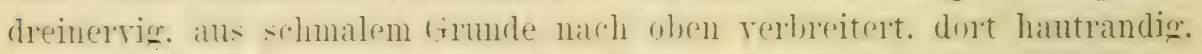

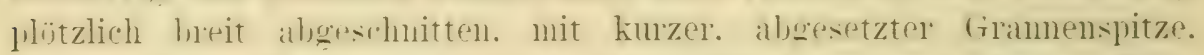

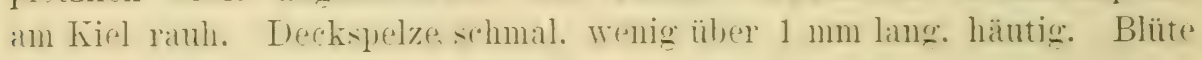
Mai bis Juli.

Auf Schutt bei Hamburg selten.

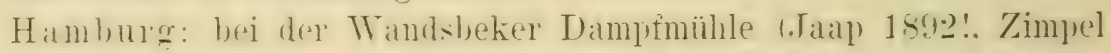
1:94-1961\%. Klosterland in Harrestehude (.J. sohmidt 1890!) am Dielsteich in Altona (Dinklage, Kausch 1885!).

Erwähnt von Prahl (Krit. Fl. 247 [1890]) als Phleum asperum Jacq.

50. ladr. 32. Phleum subulatum A. u. (ri. Syrn. Mitt.-Europ. Fl. II. 1. $1 \check{4} 4$ (1898).

๑. S.tengel zu mehreren, aufrecht oder gaknickt aufsteigend, $0,2-0.3(-0,7) \mathrm{m}$ hoch, glatt. Blattscheiden glatt oder schwach rauh, obere etwas erweitert. Blattfläche bis $4 \mathrm{~mm}$ breit, oberseits und am

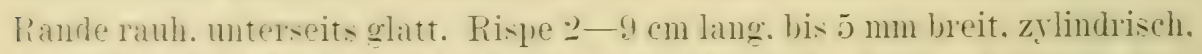
zutweilen riberwärts oder beiderseits rerschmälert. dicht. Ährchen seitlich flach geflüuckt. $2.5-3 \mathrm{~mm}$ lang. Hüllspelzen filst gleich. dreinerrig. cherwärts latutrandig. mit gewïltem hiel und kuzer spitze. zusammen- 
neigend, rauh. Deckspelze etwa 1 mm lang, breit. Vorspelze mit fünt in kleine Zähnelene des ahgestutzten Eudes anslantenden Nerven. Bliite Mai bis Juli.

Auf Schutt bei Hamburg selten.

Hamburg: (reoroswibder (Yimpel 1s!)t!). Hammerblonk (hallsels

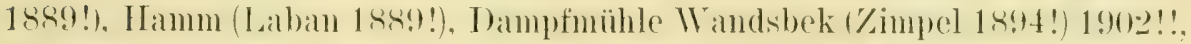
Ependorferbaum (Labin im Hb. A. Junge 1890)!), am Memonitenkirehlof bei Ottensen (C. Timm). - Nicht belegt ist eine Notiz (Ver. Naturw. Unterh. Hamb. VII. 96 [1890]), der zufolge Laban die Art im Winterhuder Bruch gefunden haben soll.

Die Ptlanze ist P. temue Schrader (so Prahl, Krit. Fl. 247 [1890]).

\section{Gattung:}

\section{Lagurus.}

L. Gen. plant. ed. 5. 34 (1754).

51. (adv. 33). Lagurus ovatus L. Spec. plant. ed. 1. 81 (1753).

$\odot$, $\odot$. Stengel zu mehreren, aufrecht oder aufsteigend, $(0,1-)$ 0,2-0,5 m hoch; kahl oder öfter behaart, kurz zottig. Blattscheiden dicht kur\% behaart, die oberen etwas erweitert. Blattfläche $3-9 \mathrm{~mm}$ breit, beiderseits dicht samtharig. Blatthäntehen etwa 2 mm lang, breit. außen dicht kurzhaarig. Rispe $2-3(-4) \mathrm{cm}$ lang, 1,5-2 cm breit, rundlich bis länglich-verkehrt-eifömig. Ährchen fohne Gramne) bis 12 mm lang. Hüllspelzen fast gleich, lineal, grammenatig ansgezogen. dicht lang weibhanig. Deckspelze ohne Gramne etwa t.j mm lang. dreinervig. alls dem Mittelnery unter der Spitze mit bis 16 mm langer Grame. ans den Seitemerven mit 2- jom langen. gramenatigen Fortsiitzen. Torselye schmal. Blüte Juni bis Juli.

Auf Schutt bei Hamburg, selten.

Hamburg: in Winterhuder Bruch (Zimpel 1s!n)!. am Eprendorfer INoor (C. 'Timm 1885 nach Prahl Krit. Fl. 249 [1890], Laban!).

23. Gattung.

\section{Polypogon.}

Desfontaines Fl: Atlant. I. 67 (1798).

1. Hüllspelzen an oberen Ende mit Einsehnitt, zwischen den Seitenspitzen begrannt. P. monspeliensis.

- Hüllspelzen allmählich in die kurze Granne verschmälert........... elongatus. 
52. (adv, 34). Polypogon monspeliensis Desf. Fl. Atlant. I. 67 (1798).

¿. Stengel zu mehreren, aufrecht, (),1-0,5 m hoch, glatt oder schwach rauh. Blattscheiden glatt oder schwach rauh, anliegend. Blattfläche heiderseits rauh, meistens $3-6 \mathrm{~mm}$ breit. Blatthäutchen bis 5 mm lang, stumpflich. Rispe $4-8(--12) \mathrm{cm}$ lang, bis $2 \mathrm{~cm}$ breit, nach oben allmählich, nach unten plötzlicher verschmälert, etwas locker, gelappt. Ährchen $2 \mathrm{~mm}$ lang. Hüllspelzen fast gleich lang, die untere breiter, beide aus der wenig ausgeschnittenen spitze mit bis $6 \mathrm{~mm}$ langer Crrame. Deckspelze nicht $1 \mathrm{~mm}$ lang, häutig; kahl, oberwärts am Rande gefranst, kurz begrannt. Vorspelze schmäler. Blüte Mai bis Juli, zuweilen später.

Auf Schutt bei Hamburg und Kiel.

Hamburg: Wollkümmerei am Reiherstieg (Zimpel 18:4!. Mohr 1×96!), Georgswärder (Zimpel 1894!). Hammerbrook (Laban 1865!, 1866!), am Ausschlägerweg (.J. Schmidt 1888!), an der Außenalster (C. Timm), Winterhude 1A. Junge Verhandl. Ver. Naturw. Unterh. VII. 97 [1890], im Herbar ohne Standort!), am Diebsteich in Altona (Brums 1886!, Hb. Prahl). Bahrenfeld 1901!!. Kiel (A. Christiansen 1907!).

schon Buek nemit P.monspulimesis von Hamburg (Hoprpe Bot. Taschenb. 91 [1801]) als Alopments prenimen (L. Spece plant. ed. 2. 90 [1762]). Ob er wirklich diese Art vor sich hatte, ist fraglich.

53. (adv. 35). Polypogon elongatus Humb, Bompl, u. Kunth Nova gen. et spec. I. 134 (1815).

2 (oder bei uns ¿ ?). Stengel zu mehreren, aufrecht, meistens $0,4-0,6 \mathrm{~m}$ hoch. glatt. Blattscheiden glatt oder deutlich rauh, die oberen schwach erweitert. Blattfläche $2-4(--7) \mathrm{mm}$ breit, rauh. Blatthäutchen bis $5 \mathrm{~mm}$ lang. spitz. Rispe locker, crelappt. 5--10 cm lang. lis $2 \mathrm{~cm}$ breit. Ährchen $2.5 \mathrm{~mm}$ lang. Hüllspelzen gleich. eimnervig. die untere über den ganzen Rücken stärker. die obsere schwächer behart, erstere mit bis $2,5 \mathrm{~mm}$ langer, stärkerer. letztere mit bis $2 \mathrm{~mm}$ langer, schwächerer Grame ans der spitze der suelze. Deckspelze wenig über $1 \mathrm{~mm}$ lang, häntig. zuweilen mit kurzer Grame ans der ausgerandeten Spitze. Blüte Angust bis Oktober.

Auf Schutt bei Hamburg selten.

Hamburg: Wollkimmerei am Reiherstieg (.J. Schmidt 18:14!. Laban 1895 !, 1896 !). - Hierher P. maritimes 4. Ber. Bot. Ver. Hamb. in Deutsche

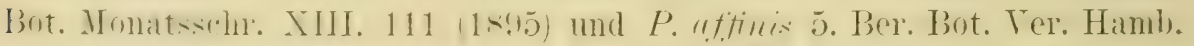
in Heimat VI. (1896). 


\section{Gattung:}

\section{Sporobolus.}

R. Brown Prodr. Fl. Nov. Holland. 169 (1810).

54. (ady. 36). Sporobolus indicus R. Brown Prodr. Fl. Nor. Holland. 170) (1810).

$\odot$ (?). Stengel zu mehreren, aufrecht, $0,4-0,6 \mathrm{~m}$ hoch, glatt. Blattscheiden eng, glatt. Blattflache (1-) $2-4 \mathrm{~mm}$ breit, öfter grefaltet, glatt. Blatthäutchen fehlend. Rispe schmal, mit anliegenden Ästen, bis $4 \mathrm{~mm}$ breit, am Girmede ofter unterbrochen, bis $15 \mathrm{~cm}$ lang. Ährchen $1,5 \mathrm{~mm}$ lang. Hüllspelzen fast gleich, die obere etwas länger, beide grin, glänzend, kahl. spitzlich. Deckspelze kahl, gramnenlos. etwa $1 \mathrm{~mm}$ ling. Vorspelze so lang wie die Deckspelze. Blïte August bis Oktober.

Auf Schutt bei Hamburg selten.

Hamburg: Wollkämmerei am Reiherstieg (Zimpel 1895!, .J. Schmidt 1896!, 1897!, Jaap 1899, Mohr 1906!).

Sporobolus capillaris Arn. ex Miq. Anal. Bot. Ind. II. 25, im 7. Ber. Bot. Ver. Hamb. in Deutsche Bot. Monatsschr. XVI. 6. 115 (1898) von der Wollkämmerei mit Autorbezeichnung "Vasny" genannt, kommt nicht vor. Das betreffende Exemplar (Hb. Zimpel!) gehört zu voriger Art.

\section{Gattung.}

\section{Agrostis.}

L. Gen. plant. ed. อ̃. 30 (1754).

1. Ährchenachse über die Blüte verlängert; Vorspelze wenig kürzer als die

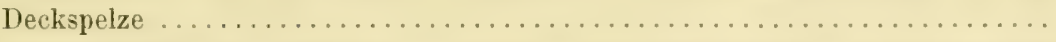

- Ährchenachse nicht verlängert; Vorspelze viel kürzer als die Deckspelze oder

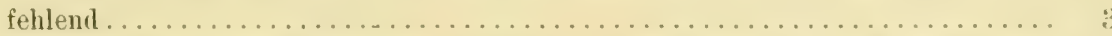

2. Rispenäste verlängert, abstehend; Ährchen $2,5 \mathrm{~mm}$ lang......... spica venti.

- Rispenäste kurz, aufrecht; Ährchen $2 \mathrm{~mm}$ lang .............. interupta.

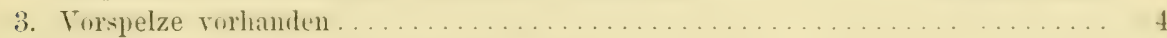

- Vorspelze fehlend oder schïppchenartig. . . . . . . . . . . . . . . 7

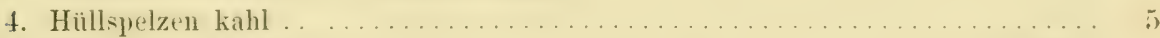

- Hüllspelzen kurzhaarig........................ verticillata.

5. Pflanze 4; Ahrehen in der Regel $2-2,5$ mm lang ................ 6

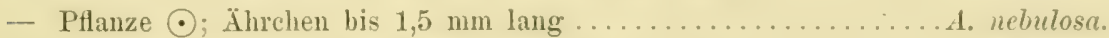

6. Blatthäutchen spitz, $2-5 \mathrm{~mm}$ lang .......................... alba.

- Blatthäutchen stumpf, kurz, selten bis $2 \mathrm{~mm} l a n g . . . . . . . . .$. . vulgaris.

7. Deckspelze an allen, selten nur einzelnen, Blüten begrannt; Ährchen $2-3 \mathrm{~mm}$

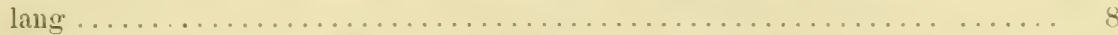

- Deckspelze gramnenlos; Ahrehen 1-1,5 mm lang .............. hiemalis. 


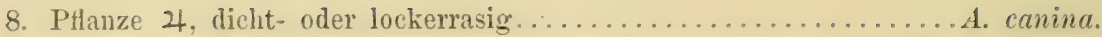

— Pflanze $\odot$; Deckspelze mit etwa $3 \mathrm{~mm}$ langer, geknieter Grame... A. retrofiracta.

Agrostis setacea Curtis Fl. Lond. VI. t. 12 (1787), von G. H. Reichenbach (Vorlesungsterz. Hamb. 20 [1881]) als ron Nolte auf den Nordfriesischen Inseln: Röm beobachtet genannt, fehlt in Noltes Herbarium und ist rou sonst niemand auf der Insel gefunden worden, so daß eine Verwechslung rorliegen muß. Reichenbach gab seine Nachträge nach Noltes Handexemplar der "Noritien". Hier hat Nolte Aira setacea für Tïm nachgetragen (Hansen hatte sie 1słf; entder.kt!). eine Hinzutügung, die Reichenbach infolge Abkürzung (A. setacer) auf Agrostis setacea bezog.

55. (sp. 13). Agrostis alba L. Spec. plant. ed. 1. 63 (1753).

4. Grundachse mit kurzen bis sehr verlängerten, zuweilen unterirdischen. Auslüutern. Stengel $(0,1-10.3-0 . \times(-1,5) \mathrm{m}$ hoch, aufrecht bis aus niederliegendem Grunde aufsteigend, dann zuweilen an den unteren Knoten wurzelnd, glatt. Blattscheiden glatt, selten an oberen Ende schwach rauh. Blattfläche $1-5(-8) \mathrm{mm}$ breit, flach oder zusammengefaltet, schlaff bis starr, beiderseits rauh. Blatthäutchen 2-5 mm lang, spitz. zuweilen eingesehlitzt. Rispe 5-15-25) cm lang, nach der Blüte schmal, mit anliegenden Ästen. ت̈hrchen 1.5--2,5 mm lang, lanzettlich. Hüllspelzen fast gleich, spitz. Deckspelze 1 -(fast)2 mm lang, häutig, in der Regel grammenlos. Vorspelze klein, zuweilen fehlend.

An Wegrändem und auf Triften, an Ablängen und in Wäldern, an Lfern und in Dünentälern durch das ganze (iebiet, anch anf den Nordfriesischen Inseln und Helgoland, häufig bis nicht selten.

Zu dieser Art zällt bei Weber (Prim. Fl. Hols. 7 [1780]) A. stolonifera \%. T.. ebenso .1. a.pma Weber (supplementum 4 [1787]) nach Exemplaren in Fröhlichs Herbar! (rerg]. Prahl Krit. Fl. 248 [1890]). Hierher gehört weiter 1. mbin rerschiedener Autoren, so z. B. bei Weber (a. a. 0. 7 [1780]), bei Hornemamm (Dansk oec. I'lantel. 3. Ulg. 1. it [1821] z. T., ebenso bei Schumacher (Enumeratio I. 2:3 [1803]) sowie bei Rafn (Fl. Damm. Holst. I. 520 [1796]).

\section{Formen.}

1. Stengel niederliegend, an den Knoten wurelnd, nur im obersten

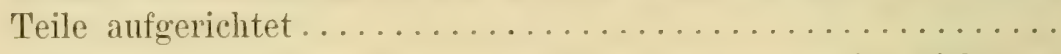

- Stengel aufrecht oder aufsteigend, nur ausnahmsweise dicht am Grunde wurzelnd ......................... 3

2. Pflanze lebhaft grün; Blätter flach:

f. prorepens Ascherson Fl. Brandenb. I. 819 (1864).

Auf feuchtem Sand- und Tonboden, an Ufern und in Gräben, in Sandausstichen und in Mergelgruben nicht selten, auch auf den Nordfriesischen Inseln und Helgoland. - 
Zuweilen finden sich Obergänge nach fo giganter, so z. B. Preetz (Ecklon 1822!), Fehmarn (Nolte 1825!), und nach f: maritima.

\section{Dazı :}

f. pauriflora Richter PI. Europ. I. 43 (1890). - Rispe wenigährig; Deckspelzen begrannt.

Diirfte im Gebiet nicht fehlen.

- Pflanze graugrün; Blätter sämtlich oder größtenteils eingerollt, starr, oft stechend:

f. maritima Meyer Chlor. Hannov. 656 (1836).

Auf feuchtem Boden am Seestrande und in den Stranddünen nicht selten, zuweilen in Menge, besonders auf den Nordseeinseln.

Tolte nemt die Form in seinen handschriftlichen Yachtrïgen als A. maritima Lam., A. frondosa Tenore, A. straminea Host, A. arenaria Gouan ron Sylt und Röm. A. alba flacescens Nolte Hb. zählt z. T'. hierher!. Hier ist jedenfalls auch $A$. alba $\delta$. syrticola $\mathrm{F}$. r. Müller (Breriarium plant. 477 [1853]) ron Ording in Eiderstedt einzuordnen.

Zerfällt:

f. Clementei A. u. Gr. Syn. Mitt.-Europ. Fl.II. 1. 176 (1899). - Stengel 2-3 dm hoch; Blattfläche eingerollt, glatt; Rispe bis $5 \mathrm{~cm}$ lang, oft zylindrisch. - So verbreitet.

f. psendopungens Lange Nat. For. Kjöbenh. II. 31 (1860). Stengel niedrig, kurzgliedrig; Blattfläche borstlich gefaltet, stechend; Blattscheiden sehr weit, gestreift. So seltener.

$\mathrm{Zu}$ dieser Form zählt A. alba $f$. subcanina Appel in Knuth F1. Nordfries. Ins. 142 (1895), die nach der Beschreibung niedrig ist und sehr schmale Blätter besitzt, die denen ron A. conina ähnlich sind.

3. PHanze lockerrasig.

- Pflanze kleine, dichte Rasen bildend, mit kurzen Ausläufern oder ausküuferlos:

f. coarctate Blytt Norsk Fl. 149 (1847).

Auf pflanzenarmem, aufgebrochenem Boden zerstreut durch das Gebiet, auch auf den Nordseeinsehn.

Schon genannt von Hornemann (Oec. Plantel. 3. Udg. I. 79 [1821]). In die Nähe dieser Form resp. in die Reihe der Ubergänge nach $f$. maritima zählt rermutlich f. flavescens Jaap (Allg. Bot. Zeitschr. IV. 1. 3 [1598]), beobachtet bei List auf Sylt, ausgezeichnet durch gelbweiße Ährchen. 
4. Stengel $0,6-1(-1,5) \mathrm{m}$ hoch, oft sehr starr aufrecht; Blätter 4-5(-8) mm breit; Rispe bis $25 \mathrm{~cm}$ lang, sehr reichährig:

f. gigantea Meyer Chloris Hann. 655 (1836).

An feuchten Orten zwischen höheren Pflanzen, in Gebüschen, in Wäldern und an quelligen Abhängen nicht selten, stellenweise häufig; auch auf Helgoland (Brody!). Zerfällt:

f. compressa A. u. Gr. Syn. Mitt.-Europ. Fl. II. 1. 173 (1899). - Ährchen (Hüllspelzen) violett überlaufen; Deckspelzen sämtlich oder z. T. begrannt. - So nicht selten.

f. silvatica A. u. Gr. a. a. O. II. 1.174(1899). - Ährchen blaß, grün; Deckspelze grannenlos. - So nicht selten. Dazul :

f. robusta nov. f. - Stengel sehr dick, bis $1,5 \mathrm{~m}$ hoch, starr aufrecht; Blätter etwas derb, abstehend; Rispe 20-25 cm lang, mit kuzen, starren Ästen. Wuchs ähnlich dem von Calamagrostis neglecta. - Angeln: Ausacker (Hansen 1863!). A penrade: Elisenlund 1909 !!.

- Stengel $(0,1-) 0,2-0,6$ m hoch, schwach; Blätter 2-4 mm breit; Rispe 5-10 cm lang:

f. gemina Schur Oestr. Bot. Zeit. IX. 48 (1859), - Häufig. Zerfällt:

f. flavida A. u. Gr. a. a. O. II. 1. 174 (1899). - Ährchen grünlich bis gelblich. - Häufig:

f. diffusa Host Gram. Austr. IV. t. 55 (1809). - Ährchen violett überlaufen. - Nicht selten. - Auch kombiniert beobachtet mit f. armata Celakorsky (Böhm. Ges. Wiss. 178 [1887]) mit aus der Mitte begrannter Spelze, so Husum: Ohrstedt Bahnhof (A. Christiansen 1911!).

56. (adv. 37). Agrostis verticillata Villar's Prosp. Fl. Dauph. 16 (1779).

4. Grundachse ausläufertreibend. Stengel $0,4-0,8 \mathrm{~m}$ lang, aus niederliegendem Grunde aufsteigend oder aufrecht, oft wurzelnd. Blattscheiden glatt. Blattfläche $4-6 \mathrm{~mm}$ breit. in der Regel flach. Blatthäutchen bis $4 \mathrm{~mm}$ lang, stumpflich, oberwärts eingeschnitten. Rispe 6-10 mm lang. mit abstehenden. rom Grunde dicht mit ährchen besetzten Ästen. Ährchenstiele kurz, 1(-2) mm lang. Ährchen 1,5(-2) mm lang. Hüllspelzen dicht weich kurzhaarig. Deckspelze etwa $1 \mathrm{~mm}$ lang. Blüte Juni bis Juli. 
Auf Schutt bei Hamburg selten.

Hamburg: Wollkimmerei am Reiherstieg (.J. schmidt 1896!), Winterhuder Bruch (Zimpel 1890!, J. Sclimidt 1893!).

57. (1). 14). Agrostis vulgaris Withering Arrang. 13: (1772).

4. (irundialse mit kurzen Ausläutern. Sitengel meistens anfrecht

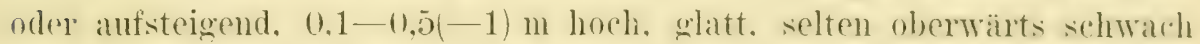
rauh. Blattscheiden glatt. Blattläche $(1-) 2-6 \mathrm{~mm}$ breit, oberseits deutlich rauh, unterseits schwach rauh bis glatt. Blatthäutchen sehr

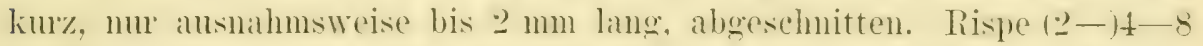
(--15) cm lang. mit auch nach der Blïte abstehenden Ästen. Ïhrehen (1.5-)2 mm lang, an bis ó mm langen stienen. Hüllspelzen kahl, am Kiel oberwärts (im oberen Drittel) gezähnelt-1auls. Deckspelze bis ctwas üb)er $1 \mathrm{~mm}$ lang, häutig. selten begrannt. Torspelze halb so lang wie die Deckspelze. Blïte Juni bis August, zuweilen auch später.

Auf Wiesen, 'Triften, Heiden und Mooren, in Wäldern, Gebüschen und an Abhängen häutig. auch auf Föhr (Schiöz) zerstreut!!. Amrum (Euchenau). Sylt (Schiötz. Prahl!) häufig!!, R ïm (Ramkiaer) und Helgoland (Hallier, Brody!) häufig:

Die erste Erwähmung der Art für das Gebiet gibt Oeder (Flora Danica 163 [1764]) als A. capillaris. Webers (Prim. Fl. Hols. 7 [1780]) A. stolonifera zählt z. T. hierher. Als A. vulgaris wird die Pflanze von Hornemann (Oec. Plantel. 3. Udg. I. 74 [1821]) erwähnt.

A. pumila L. bei Weber (a. a. 0. 7 [1780]) (nach L. Mant. I. 31 [1767]) zählt hierher als durch Tilletia decipiens (Pers.) Körner deformierte Form. Derart befallene Pflanzen sind bei uns häufig.

\section{Formen.}

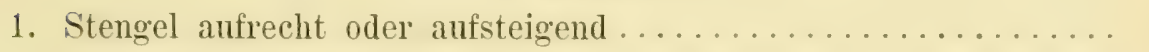

- Stengel am Grunde oder völlig niederliegend, an den Knoten wurzelnd:

f. stotonifera Koch Syn. Fl. Germ. Helv. ed. 1. 782 (1837).

Auf pflanzenarmem (feuchtem) Boden durch das Gebiet zerstreut; auch auf Helgoland.

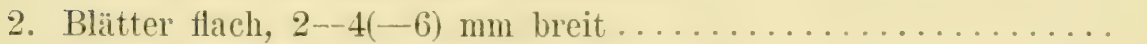

- Blätter borstlich gefaltet oder eingerollt:

f. humilis A. 1. Gr. Syn. Mitt.-Europ. Fl. II. 1. 181 (1899). Pflanze niedrig, büschelig-rasig, $10-20 \mathrm{~cm}$ hoch; Blatthäutchen kürzer als beim Typus.

Auf Moor- und Sandboden besonders im Heidegebiet. sonst seltener. 
Als Übergangsform mit gleichem Wuchse, aber flachen Blättern, ist $f$. pumila Hornemann (Oec. Plantel. 3. Udg. I. 77 [1821]) anzusehen nach Abbildung Flora Danica t. 1802 (1825). Derartige Übergänge sind verhreitet.

3. Stengel $(0,2-) 0,3-0,5(-1) \mathrm{m}$ hoch; Rispe mit locker entferntstehenden Ährchen, ihre Äste unterwärts ohne Ährchen....... 4

- Stengel 0,1-0,2(-0,3) m hoch; Rispe kurz mit sparrigen Ästen und genäherten Ährchen, ihre Äste vom Grunde mit Ährchen besetzt:

f. contracta nov. f. - Blätter mit kurzer Blattfläche; Hüllspelzen tief violett.

Hamburg: auf Schutt am Lehmweg in Eppendorf (Laban 1889!); im Winterhuder Bruch (Laban 1890! als A. Autcis) and Winterhude (Zimpel 1893!, auch als Ubergang nach $f$. stolonifera). Husum: zwischen Schwesing und Ahrenviöl (A. Christiansen 1909!).

4. Spelzen violett gefärbt:

f. gemina Schur Oestr. Bot. Zeit. IX. 45 (18599). - Häufigste Form.

- Spelzen bleich gefärbt:

f. pallescens nov. f. - Stengel aufsteigend, fast $1 \mathrm{~m}$ lang, unter der Rispe glatt. Blattfläche schlaff, stark verlängert. Rispe bis $15 \mathrm{~cm}$ lang, mit locker stehenden, seitwärts gerichteten, zum Teile rauhen, bis $5 \mathrm{~cm}$ langen Ästen. Hüllspelzen schmäler als beim Typus, mit grünem Mittelstreif, hautrandig.

Selten. - Stormarn: Gebüsch am Ahrensfelder Teich bei Ahrensburg!!. Eckernförde: Behrenbrook (A. Christiansen!). - Eine Übergangsform Hamburg: bei der Wollkämmerei am Reiherstieg (Zimpel!) mit sehr schlaffer, kurzästiger Rispe.

Die Abart ist mit f. umbrosa Schur (Oestr. Bot. Zeit. IX. 47 [1859]) verwandt, unterscheidet sich aber durch den aufsteigenden, höheren, nicht rauhen Stengel, breitere Blätter und nur zum Teil rauhe Rispeniiste.

\section{Mißbildungen.}

f. m. vivipara Rehb. Je. Fl. Germ. et Helv. I. t. XXXIV. fig. 1429 (1850). - Ährchen in Laubsprosse auswachsend.

Husum: Schwesing! und (wenig charakteristisch) T'ondern: Enge (A. Christiansen!).

58. (11. 15). Agrostis canina L. Spec. plant. ed. 1. 62 (1753). 4. Gimulachse dicht bis lockerrasig, selten ansläufertreibend. Stengel 
$0,2-0,4(-0,6)$ m hoch, aufrecht oder aufsteigend, glatt. Blattscheiden glatt. Blattfliche der unteren Blätter meistens eingerollt, der oberen meistens flach und bis $2(-3,5) \mathrm{mm}$ breit, glatt oder beiderseits rauh. Blatthäutchen bis $3 \mathrm{~mm}$ lang, spitzlich. Rispe $5-10(-15) \mathrm{cm}$ lang.

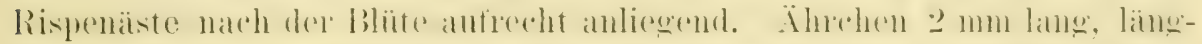

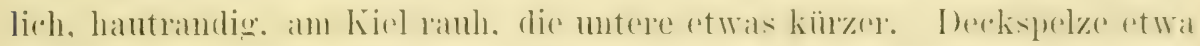
$1,5 \mathrm{~mm}$ lang, aus der Nitte des Rückens in der Regel begramnt. Granne meistens 2-2,5 mm lang, gekniet. Vorspelze in der Regel fehlend. Antheren wenig über $1 \mathrm{~mm}$ lang. Blïte Juni bis Juli.

Auf Mooren und (feuchten) Heiden sowie anf Moor- und sumpfigen Flußwiesen im mittleren Gebiet nicht selten, sonst mehr zerstrent, auch auf Föhr: Nieblum (Kertelhein), Amrum und Sylt auf Heiden und in Dünentälern; bei Hamburg selten auf Schutt!.

Die erste Erwähnung findet die Art bei Weber (Prim. Fl. Hols. 7

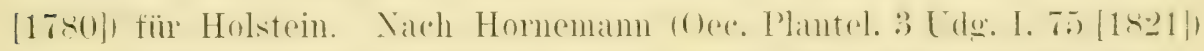

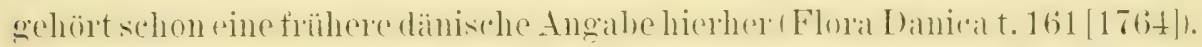

\section{Formen.}

1. Deckspelze mit geknieter, das Ährchen überragender Gramne... 2

- Deckspelze mit gerader, das Ährchen nicht überragender Grame oder grannenlos ......................... 5

2. Pflanze ohne Ansläufer ......................

- Pflanze mit (zuweilen sehr verlängerten) Ausläufern:

f. stolonifera Blytt Norges Flora 81 (1861).

Selten. - Lauenburg: an der Aue im Sachsentrald (Kohlmeyer, Hb. Luibeck!). Stormarn: im Hagenmoor bei Ahrensburg!!. Angeln: Husby (Hansen!).

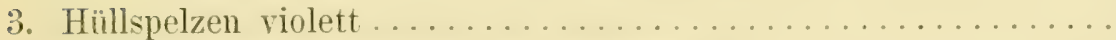

- Hüllspelzen gelbbräunlich bis gelbgrïnlich:

f. varians A. u. Gr. Syn. Nitt.-Europ. Fl. II. 1. 185 (1899).

Sehr zerstrent. - Hamburg: Hasselbrook (J. A. Schmidt 1868!). Lübeck: Grönaner Heide (Häcker 1845!). Kiel: am Einfelder See (Hennings 1876!), M[önkeberger Moor (A. Christiansen 1908!). Husum: Bohmstedter Busch (A. Christiansen!). Röm: 'Twismark (J. Schmidt!) und Kongsmark $1904 !$ !

4. Pflanze grasgrün; Stengel nicht starr; Blätter oft etwas schlaff: f. gemina Godr. u. Gren. Fl. Fr. III. $48+$ (1856). - 'Typische Form.

Da\%11:

f. arida Schldl. Fl. Berol. I. 4ó (1823). - Stengelblätter wie die Grundblätter mit eingerollter Blatt- 
fläche. - Segeberg: Bebenseer Moor!!. A mrum: Norddorf!!.

f. uliginosa A. Christiansen nor. f. - Grundblätter flach wie die Stengelblätter. - Kiel: am Tröndelsee! und am Langensee (A. Christiansen).

- Pflanze graugrün, in allen Teilen starr:

f. vineulis A. u. Gr. a. a. O. II. 1. 185 (1899).

Selten. - Sylt: IIorsumheide!! - Übergänge z. B. Segeberg: Tensfelder Moor!! und Angeln: Moor bei Weesries!!

5. Granne rorhanden, $0, \check{0}-1 \mathrm{~mm}$ lang:

f. pudica Döll Rhein. Fl. 108 (1843).

Im Gebiete unsicher. Sonder zählt die Form ohne Standort auf (Fl. Hamb. 39 [1851]): „Granne selten sehr kurz".

- Granne fehlend:

f. mutica Gaud. Fl. Helv. I. 172 (1828).

Selten. - Lauenburg: Grönauer Heide (Häcker). Stormarn: Ladenbeker Tannen bei Sande (J.Schmidt1891!). Hamburg: Eppendorfer Moor (J. A. Schmidt 1877!), Borsteler Moor (C. Timm nach Knuth Fl. v. Schlesw.Holst. 753 [1887]). Dithmarschen: am Ecksee bei Tellingstedt (J. Schmidt!). Husum: Bohmstedter Busch (A. Christiansen 1912!). Angeln: Rüllschau (J. Lange 1863!). Röm: Kongsmark!!.

Die Form ist mit der vorhergehenden durch Übergänge verbunden. Nicht alle Spelzen sind grannenlos; eine mehr oder weniger croße Minderzahl zeigt eine kurze Granne, bei der Pflanze von Röm z. B. ron 114 untersuchten Deckspelzen 47, wälurend 67 grannenlos waren. Diese Pflanze zählt also zu f. variiflora Waisb. (Oestr. Bot. Zeit. XLV. 109 [1895]).

59. Iadr. 3-1. Agrostis hiemalis Britt.. sternb. et Poggenl. Prelim. Catal.

I. I. 68 (1888).

¿. Stengel zu melneren. in der Regel aufrecht, seltener anfsteigend. 11.2-0,4 un hocll, zierlich. glatt. kahl. Blattscheiden lang gestreckt, eng, rlatt. Plattläche schmal. etwa $1 \mathrm{~mm}$ breit. flach oder oft eingerollt, ranh. Blatthäutchen bis $2 \mathrm{~mm}$ lang. spitz. Rispe locker, bis $20 \mathrm{~cm}$ lang, mit bis zu arht gemeinsan entsungenden. selır teinen. rückwärts rauhen. bis 7 cn langen Ästen. Ährchen $1-1.5(-2)$ mm lang. Hüllspelzen ungreich. untere etwa $1 \mathrm{~mm}$. obere etwa $1.5 \mathrm{~mm}$ lang. beide am hiele rauh. I)eckelelze etma $1 \mathrm{~mm}$ lang. spitzlich. glatt. grannenlos. Torspelze fehlend. Bliite Juli bis August. 
An Fischteichen selten.

Stormarn: am Kupferteich bei Poppenbüttel 1905!! (mit Carex vulpinoidea Rich.).

Synonym ist A. scabra Willd.

60. (adv. 3!)). Agrostis mebulosa Boiss. u. Rent. Diagn. 20 (1842).

$\odot$. Rasig. Stengel zu mehreren, aufrecht oder aufsteigend, 0,1-0,t m hoch, glitt. Blattscheiden rauli. Blattfläche rauh, bis a) mu breit. Blatthäutehen bis + mm lang, oberwäts zerrisisen. Rispe $5-15$ ('m lang, reich verzweigt. Ähroh('n lang gestielt, 1,5-2) mm lang. Hüllspelzen griunlich, gleich lang, stumpflich. I)eckspelze etwa 1 mm lang. stumpf. gramenlos. Torspelze länger als die Decksjelze. Blüte Juli bis September.

Auf Schutt bei Hamburg selten.

Hamburg: Winterhuder Bruch (J. Schmidt 1890!; Ber. Deutsche Bot. Ges. IX. 126 [1891]).

61. (adv. 40). Agrostis retrofracta Willd. Funmeratio I. $14(180 !)$.

$\odot$. Stengel zu mehreren bis vielen, in der Regel aufsteigend. seltener aufrecht, 0,3-0,4 m hoch, glatt oder öfter schwach rauh, kahl. Blattscheiden glatt, kahl, die oberen oft etwas erweitert. Blattfläche etwat 2(-3) mm breit, unterseits glatt, oberseits schwach raul. Blatthäutchen bis 8 mun lang, spitzlich. Rispe $10-15(-20) \mathrm{cm}$ lang, locker. mit bis 11 cm langen, schwach rauhen Ästen. Ähchen 2,5(-3) mm lang. Hüllspelzen fast gleich, mit grünem, rauhem Kiel und häutigem Rande, spitz. Deckspelze $1 \mathrm{~mm}$ lang, stumpf oder @-- tzähnig, am Grunde und Rücken kurzhatrig, mit in der Mitte inserierter, geknieter, etwa $3 \mathrm{~mm}$ langer Granne. Vorspelze fehlend. Blüte (bei uns) Juni bis August.

Auf Schutt bei Hamburg selten.

Hamburg: bei der Wollkämmerei am Reiherstieg (J. Schmidt $1895 !)$.

Hierher zählt Avena myriantha in verschiedenen Hamburger Herbarien, aber nicht Trisetum myrianthum Parlat., sowie auch Mühlenbergia sp. in Deutsch. Bot. Monatsschr. XVI. 115 (1898). Synonym sind A, aemula R. Brown und A. Forsteri Kunth.

62. (qu). 7). Agrostis spica venti L. siper. plant. e(d. 1. 61 (1753).

$\odot$. Stengel zu mehreren, aufrecht oder seltener aufsteigend, $(0,2-)$ 0,4-1 m hoch, glatt. Blattscheiden meistens glatt. Blattflache $1-t \mathrm{~mm}$ 
Weit. raulı. Blatthäutchen $1-6 \mathrm{~mm}$ lang. zerschlitzt. Rispe (5-) $10-20 \mathrm{~cm}$ ling. nit wh verlängerten. teinen Ästen. Äste nehr nder weniger ab-

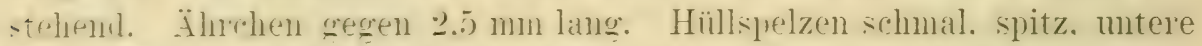

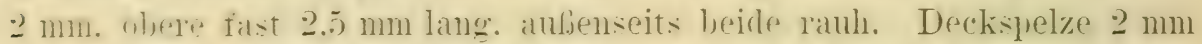
lang. stälor rauh als die Hüllspelzen. mit lis 7 mm langer. gerader

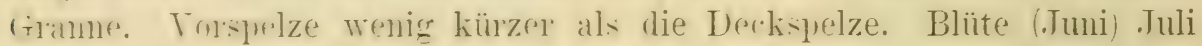
bis August.

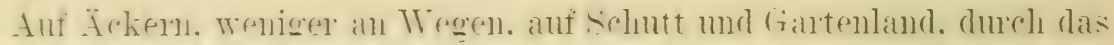

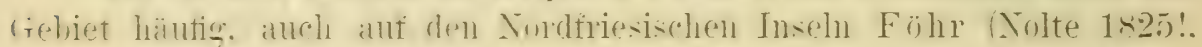
schivitz !! Sylt Ischiritz!!! und Röm PRaunkialer sowie auf Helgoland:

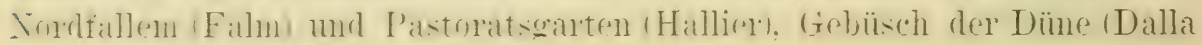
Torre). Acker im Oberland 1912!!.

Teber nennt die Art zuerst aus dem Gebiet (Prim. Fl. Hols. 6 [1780]).

\section{Formen.}

f. nun nor. f. - Stengel einfach, 5-10 cm hoch; Rispe kurz, wenig-

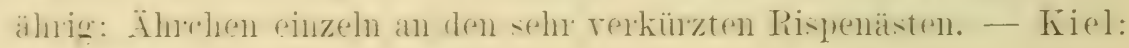
Achterwehr (A. Christiansen!).

\section{Farbenformen.}

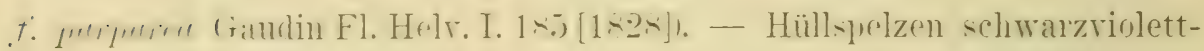
braum überlaufen. - Kiel: auf Schutt (A. Christiansen 1908!).

63. adir +11 . Agrostis interrupta L. systema ral. 10. $87211759 \%$.

○. Stengel zu mehreren. meistens aufrecht, $0,2-0,6 \mathrm{~m}$ hoch, glatt.

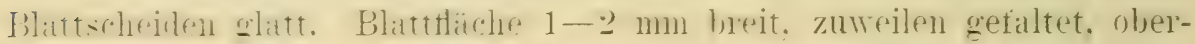

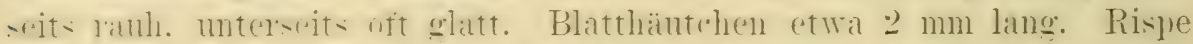

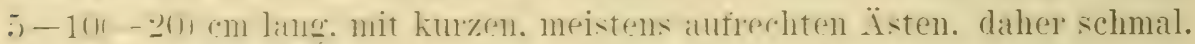

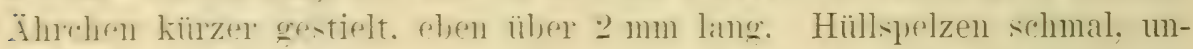
gleich, rauh. Deckspelze fast $2 \mathrm{~mm}$ lang, rauh, mit bis $10(-15) \mathrm{mm}$ langer Grame. Blüte Juni bis Angust.

Auf Schutt bei Hamburg selten.

Hamburg: bei der Wandsbeker Dampfmïhle (J. Schmidt 1894!, Zimpel 189t!).

Webers Angabe (Prim. Fl. Hols. 6/7 [1780]) dürfte kaum richtig sein. Schon Hornemann rermutet (Oec. Plantel. 3. Udg. I. 74 [1821]) in Webers Pflanze A. spica venti. Auf diese Art beziehen sich auch die Angaben Labans (Fl. r. Holst. 225 [1866]) (Fl. r. Hamb. 4. Aufl. 195 [1887]) sowie die ron A. Junge (Verhandl. Ver. Nat. Unterh. VII. [1890]); die eingesehenen Pflanzen sind A. spica renti! 
26. Gattung.

\section{Calamagrostis.}

Adanson Fam. II. 31 (1763).

1. Hüllspelzen lanzettlich, wenig länger als die Deckspelze, die obere etwas länger

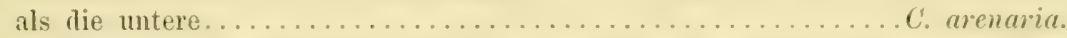

- Hüllspelzen lanzettlich bis lineal, beträchtlich lïnger als die Deckspelze, die untere linger als die where.

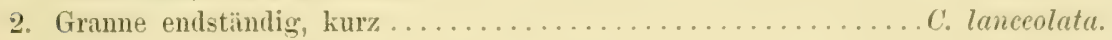

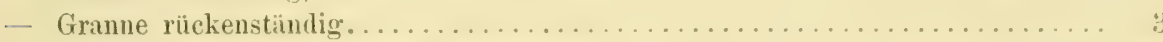

3. Gramne gerade oder schwach gekrümmt ..................... 4

- Grame deutlich gekniet, die Hüllspelzen überragend......... arundinacea.

4. Deckspelze am Grunde fünfnervig........................

- Deckspelze Ireinervig; Hüllspelzen gramenartig spitz............ epigeios.

5. Haare der Ährchenachse rings gleichmäBig verteilt, die Deckspelze an Lünge

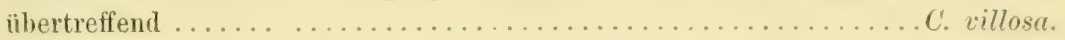

- Haare der Ährchenachse jederseits der Deckspelze in zwei Gruppen dichter gedrïngt, kürzer als die Deckspelze.................... neglecta.

64. (s). 16). Calamagrostis lanceolata Roth Tent. Fl. (ierm. I. $34(17 s-4)$.

4. Grundachse mit kurzen Ausläufern. Stengel 0,7-1,o $\mathrm{m}$ hoch, glatt oder (besonders oberwäts) schwach rauh. Blattscheiden glatt oder sehr schwach rauh. Blattflache $2-6 \mathrm{~mm}$ breit, flach oder gefaltet. beiderseits rauh. Blatthäutchen etwa $2 \mathrm{~mm}$ lang, stumpf. Rispe $10-20 \mathrm{~cm}$ lang, locker, schlaff. Ährehen schmal, 4-5(-6) mm lang. Hüllspelzen ungleich lang, die untere $4-5(-6) \mathrm{mm}$ lang, die obere etwa $0.5 \mathrm{~mm}$ kürzer, beide spitz ausgezogen. Deckspelze etwa $2.5 \mathrm{~mm}$ lang. im wheren Teile häutig, mit endstandiger, gerader, bis 1 selten 20) mm langer. die' reitenspitzen der Deckspelze nicht oder wenig (selten deutlich) ïber'agender (iramne. Haare der Ährehenachse kürzer (etwa $2.5 \mathrm{~mm}$ ) und länger retwa $3 \mathrm{~mm}$ ), rings glejchmäßig verteilt. Blüte Juni bis Juli (August).

In Nooren, in Sümpfen, an Ufern, auf feuchten Wiesen und in Gebüschen häufig oder nicht selten, doch auf den Xordfriesischen Inseln nur auf Amrum: südlich ron Vorddorf!!. Sylt: ostlich ron Kampen (Alpers) und Röm (Prahl 1904!): bei Kongsmark und zwischen Kirkeby und Lakolk (Jaap).

Die erste Erwähnung gibt Weber (Prim. Fl. Hols. 10 [1780]) als Arundo calamagrostis (nach L. Spec. plant. z. T.) und an gleicher Stelle als Arundo canescens (nach Herbarexemplaren; rgl. Nolte Nor. Fl. Hols. $11[1826])$.

\section{Formen.}

1. Hüllspelzen mit grünem Mittelstreif. sonst violett oder violettbrann: f. typica Beck Fl. Nied.-Österreich I. 61 (1890). - Hüllspelzen $4-5(-6) \mathrm{mm}$ lang. Granne bis $1 \mathrm{~mm}$ lang, kürzer, so lang: oder wenig länger als die Deckspelzenspitzen. 


\section{Dazu:}

f. macrathera Prahl Mitt. Gatt. Calamagr. 6 (1902). - Deckspelze auf $1 / 8-1 / 4$ ihrer Länge gespalten; Granne bis viermal so lang wie die Seitenspitzen.

Zerstrent; z. B.: La uenburg: am Gardensee (Prahl!). Hamburg: beim Alsterkrug und Eppendorf (Laban!), Borsteler MIoor (Zimpel!). Lübeck: Schellbruch (Häcker!, Kleinfeldt!). Angeln: Silberstedt (L. Hansen!), Wattschaukrug (Hansen!), Glücksburg!!. Hadersleben: Stokkerhoved (Prahl!).

Pflanzen dieser Form waren es z. T., welche Nolte für C. villosa Mutel (C. Halleriana [Gaudin] P. B.) gehalten hat!.

f. Typacrathera Torges Bot. Ver. Thüringen N. F. XVII. 94 (1902). - Granne dicht unter dem Spitzeneinschnitt der Deckspelze rückenständig.

Hamburg: Eppendorfer Moor (Hb. Lübeck nach Torges).

f. r(umiflora Prahl a. a. 0.7 (1902). - Stengel an den Knoten mit rispentragenden Ästen.

Hadersleben: Pamhoeler Wald (Prahl 1902!).

- Hüllspelzen grïn. Weibhautrandig oder am Rande ganz schwach violett überlaufen:

f. rnusere Prahll Krit. Fl. 249 (1890). - Hüllspelzen ganz bleich.

Zerstreut, z. B. Lauenburg: am Schmalsee bei Mölln (Friedrich!)!!, Sachsenwald!!. Hamburg (Sonder!): Eppendorfer Moor (Kohlmeyer, Hb. Lübeck!). Stormarn: Ahrensfelder Teich bei Ahrensburg!!. Lübeck: erste Fischerbude (Prahl 1889!). Eutin: Süsel (Weber als dnundo canescens), am Krummensee (Erichsen 1888!). Plön: Hornsmühlen!!. Kiel: Schönberg (J. J. Meyer 1823!). Angeln: Silberstedt (Hansen), Husbyries (Hansen!). Sonderburg: Gravenstein (Prahl 1889!). A pen rade: Hostrupholz!!. Itzehoe: Lockstedter Lager (Prahl). Dithmarschen: mehrfach bei Burg (J. Schmidt!). Husum: Immenstedter Holz (A. Christiansen!). Tondern: Hogelund und Fresenhagen bei Leck (Hansen).

Eine Übergangsform zwischen f.typica und f.canescens ist:

f. viviris Torges Bot. Ver. Thüring. N. F. VII. 21 (1895)。 Hüllspelzen am Rande schwach violett überlaufen.

Z.B. Stormarn: am Stenzerteich beiTrittan (Nolte!), AlrensfelderTeich!!. Neumünster: Brokenlande(Poulsen, Hb. Kopenhagen!). Kiel: Düsternbrook (Moldenhawer 1823!). Apenrade: am Aagsee!!. 
Calamagrostis purpurea Trinius Gram. unifl. 219 (1824) ist im Gebiete nicht gefunden worden. Die Berichtigung der Bestimmung der C. villosa aus Angeln, die J. Lange

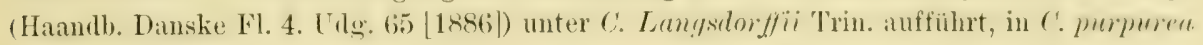
Trin. (Rettels. og Tilföjels. 7 [1897]) (=C.pleragmitoides Hartm.), wirl von 'T'orges nach Ascherson und Graebner (Sym. Mitt.-Europ. FI. II. 1. 202 [1899]) bestritten. Ist ('. pripuren eine der Arten mit endständiger Granne, so kann die Pflanze von Husby in Angeln unmöglich zu ihr gehören, denn an einer Reihe von mir gesehener Exemplare war die Granne stets dentlich rückenständig. Im Herbar J. A. Schmidt (Bot. Huseum Hamb.) $\operatorname{lag}$ indes auf gleichem Bogen mit C. villosa die schon erwähnte $f$. macrathera von C. lanceolata, so dạ es nicht ausgeschlossen ist, da B Almquist, der die Umänderung der Langeschen Angabe veranlaßt hat, nicht C. villosa, sondern die erwähnte Form vor sich gehabt hat, welche durch ihre Gramnenlänge und -stellung an C. purpurea erinnert.

\section{5. (sp. 17). Calamagrostis villosa Ifutel Fl. France IY. 41 (1837).}

4. Grundachse mit bis sehr langen Ausläufern. Stengel $0,5-1 \mathrm{~m}$ hoch, einfach, glatt. Blattscheiden kahl oder schwach rauh, unter der Blattläche jederseits mit einem Büschel kurzer Haare. Blattfläche 2-5 mm breit, rauh. Blatthäutchen kurz, breit. Rispe bis $15 \mathrm{~cm}$ lang. Hüllspelzen t-5 mm lang, spitz, lanzettlich, grün, oberwärts violett. Deckspelze etwa 2,5 mm lang, begrannt. Granne rückenständig, in oder nahe unter der spelzenmitte entspringend, die Deckspelze etwas überragend. Haare der Ährchenachse von wenig mehr als Deckspelzenlänge. Blüte Juli bis August.

An Erdwällen sehr selten.

Angeln: beim Wattschaukrug bei Husbyries: am Fussteige zwischen Lassens und Hansens Haus (L. Hansen 1867!).

Zahlreiche Angaben dieser Art liegen für unser Gebiet vor. Alle aber, mit Ausnahme der eben angeführten, sind sicher falsch oder doch ganz unsicher. Mehrere aus der Hamburger Flora genannte Fundorte gehen auf Sickmann und Hübener zurück. Ersterer gibt (Enumeratio 8 [1836]) die Bemerkung: "Reinbek. Wor Wittenbergen (Steetz)" unter C. Halleriana DC. (synonym). Unter demselben Namen führt Hübener die Art an (Fl. v. Hamb. 493 [1847]: „im Hammerbrook, in den Wiesen am Hammerdeich und bei der Blauen Brücke; am Elbufer zwischen Wittenbergen und Blankenese". Sonder berichtigte (Fl. Hamb. 40 [1851]) in C. lanceolata $f$. canescens. Trotzdem erscheint C. Halleriana DC. mit dem Fundortsvermerk Hamburg oder genauerex Bezeichnung wieder in allen vier Auflagen der Hamburger Flora von Laban und in desselben Verfassers Flora ron Holstein (224 [1866]). Der Fundort Hohenwestedt (Hennings in Nat. Ver. Schlesw.Holst. II. 1. 145 [1876]) ist unbelegt; es dürfte sich um C. lanceolata gehandelt haben. Auch Koch erwähnt die Pflanze aus Holstein (Synopsis Deutsch. u. Schweiz. Fl. 784 [1838]) und wenig später: "Yon Holstein durch das nördliche Deutschland" (Syn. Fl. Germ. et Helv. ed. 2. II. 905 [1814]), während bei ihm Noltes Fundort Lübeck (Nov. Fl. Hols. 11 [1826]) für Amulo pseudophragmites Haller fil. (三 C. psendophragmites Baumg, = C. littorea P. Beaur.) fehlt. Die Bemerkungen Kochs bilden wahrscheinlich die Grundlage der von Prahl erwähnten Angabe Garckes, der die Pflanze als am Meeresstrande von Holstein bis Pommern vorkommend nannte. Nur eine Wiederholung ist die Aufnahme der Art-bei Borchmam (Fl. v. Holst. 71 [1856]). Ein neuer Irrtum liegt in 
der Bestimmung ron Pflanzen rom Rönner Holz bei Kiel als C. Halleriana DC. durch Ohl: es handelt sich um C. lanceolata!. Die älteren Irrtümer scheinen schon L. Reichenbach bekannt gewesen zu sein, denn er verneint das Vorkommen ron C. villosa unter dem Srnonym C: nutans Saut. (Rchb. Fl. Germ. excurs. 26 [1830]) für Holstein (Jcones Fl.

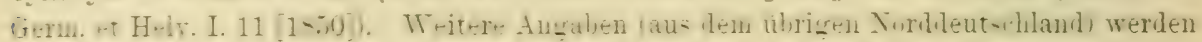
Ton Prahl besprochen (Archir Naturg. Mecklenb. LIII. 170/176 [1899]).

66. (s). 1h). Calamagrostis neglecta Pal. Beall Agrast. 157 (1812).

7. Crmudarlise mit wenigen. langen Ausläufern. stengel autrecht.

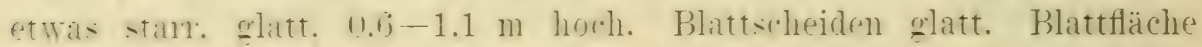
1-4 mm liwit. leiderefits stark rauh. Blatthäuthen $1-3-41$ mm lang.

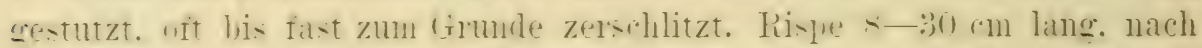

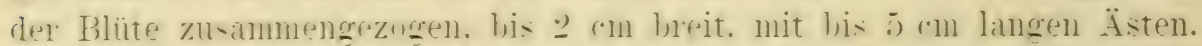

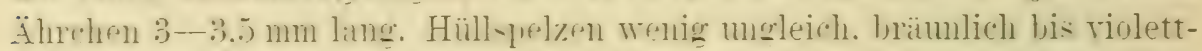

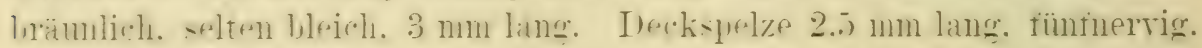

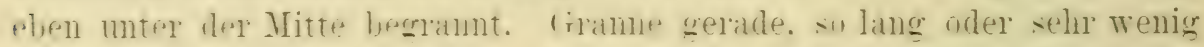
lianger als die Spelzenspitzen. Vorspelze 1,5-2 mm lang. Haare der

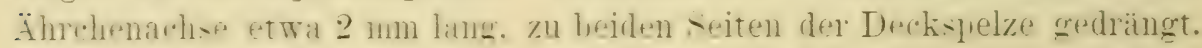
Blüte Juni bis Juli.

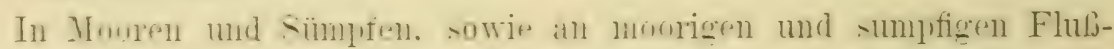
und Seeufern im südöstlichen Gebiet sehr zerstrent bis zur Eider.

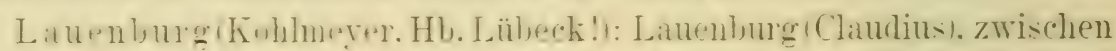

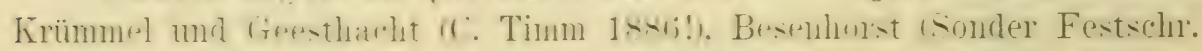

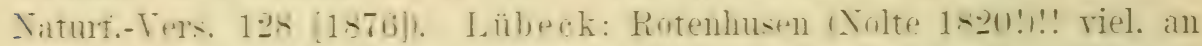

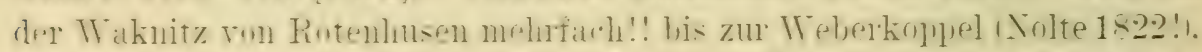

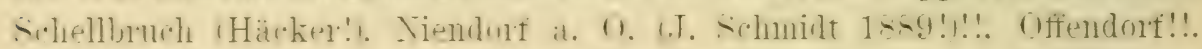
Entin: Siiseler Moor (Nolte 1821!). Preetz: am Lankersee bei Külıren (A. Christiansen 1911!). Kiel: am Drecksee (Ohl 1892!), bei Maruten-

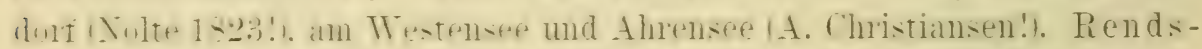

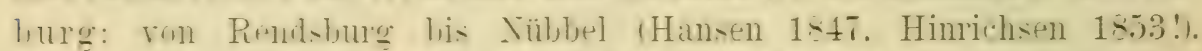
Itzehre i.J. .T. Mtyer 1817!): Kellinghuen Nolte. Hl, Kopenlagen!)

Die erste Totiz aus dem Gebiete erscheint bei Hornemann (Vid. Crlsk. phys Shrift. I. Ifeel. I. Haette $194[1 \leq 21]$ für Lanemburg: es folgt Tolte (Nor. Fl. Hols. 12 [1826]).

Der Standort: Eppendorfer Moor (Sickmann, Enumeratio 8 [1836]), ron Laban aufcenommen, ist zu streichen; ein Exemplar, ron Sonder als Arundo stricta bezeichnet, ist

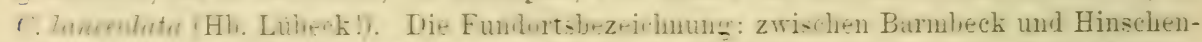

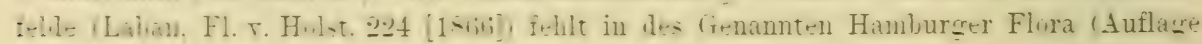
ron 1857). In der Flora Danica (t. 1503 [1825]) steht Schlutup als Fundort; Belege ron dort fehlen. An gleicher Stelle wird auch ..Rothenhause in Lauenburg aufgeführt.

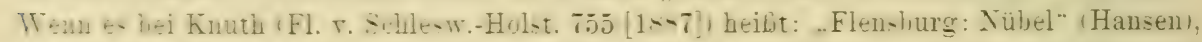
so hat eine Terlegung des Rendsburger Standorts stattgefunden, auf den (am Nordufer der Eider gelegen) die Bemerkung Reichenbachs gehen dürfte „Schleswig" (Jcon. Fl.

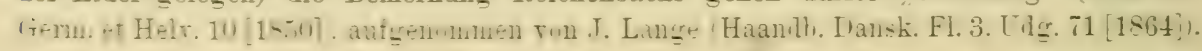




\section{Formen.}

f. vivilis Torges in A. u. Gr. Syn. Mitt.-Europ. Fl. II. 1. 208 (1899). Hüllspelzen hellgrïnlich.

Lübeck: Schellbruch (Häcker), Niendorf a. O.!!.

f. interupta Prahl in 'Torges 'Thür. Bot. Ver. N. F. VIII. 13 (1896). Rispe unterwits sehr locker, weit unterbrochen.

Im Gebiete Ubergangsformen mehrfach.

\section{Mißbildungen.}

f. m. bractecta A. Christiansen nov. f. - Rispe am Grunde mit laubigem 'ragblatt.

Kiel: bei Marutendorf am Westensee (A. Christiansen!).

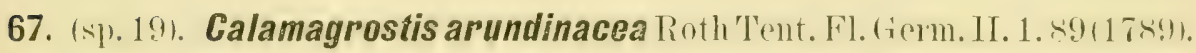

4. (irundalehse mit kurzen Ausläufern mod dicht (fast rasig) stehenden Stengeln. Stengel 0,6-1,6 m-hoch, aufrecht, glatt oder dicht unter der Rispe rauh. Blattscheiden glatt oder schwach rauh, in der Regel sämtlich kahl. Blattfläche $3-8(-10) \mathrm{mm}$ breit, rauh. Blatthäutchen $1-2 \mathrm{~mm}$ lang, stumpf. Rispe 8-25 cm lang, locker, auf aufrecht wenig abstehenden Ästen. Ährchen 4,5 mm lang. Hüllspelzen breit, fast gleich lang, spitzlich, eimnervig. Deckspelze 3,5 mm lang, begrannt. Granne im unteren Viertel der Spelze rückenständig, $6-7 \mathrm{~mm}$ lang, gekniet.

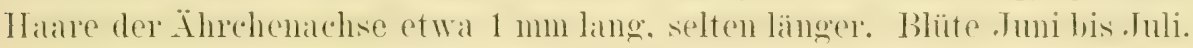

In Wäldern und Gebüschen auf in der Regel trockenem Boden zerstrent in Lauenburg und im angrenzenden storman: sonst nur selten in Heidegebiet.

La a unburg: vom Fortkrug bei Langenlehsten (Nolte 1821!), Mölln

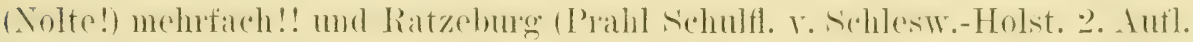
27 [1900]) bis Börnsen (Sonder) und Stormarn: Reinbek (Sonder. Hb. Lüberek!) mul Trittau (Thun. Hb. Lübeck!): in (ler Hahnheide (Hansen)

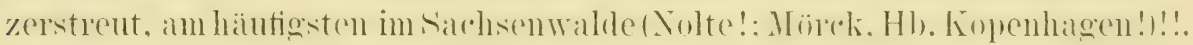
Dithmarschen: bei Burg (J. Schmidt 1899!) 1!01!!. Rendsburg: Hohn (A. Christiansen!). Flensburg: Walsbüll (Prahl!). Toudern: Hogelund bei Leck (Nolte 1833!).

Schon Weber nemnt die Art als Armelo Arrostis (Prim. Fl. Hols. 10 [1780]) nach Agrostis amminacea L.

Bei Klatt (Fl. y. Lauenb. [1865]) kommen als Standorte dieser Art die Oberschleuse bei Mölln und Tüschenbek vor; nach Friedrich (Fl. r. Lübeck 42 [1895]) gehören die Exemplare im Herbarium Klatt zu C. lanceolata. Sickmanm (Enumeratio 8 [1836]) liat Wellingsbüttel im Alstertal als Fundort; danach macht Laban (Fl. v. Hamb. 3. Aufl. 175 [1882]) seine auch in die "Flora von Holstein" (224 [1866]) schon früher aufgenommene 
eine Angabe, der sich eine zweite: Niendorf bei Hamburg zugesellt. Beide Angaben sind unglaubwürdig; Belege fehlen. Lienau sagt (Fl. Fürstent. Lübeck 7 [1863]) für das von ihm besprochene Gebiet: "Soll vorkommen". Wo, ist nicht festzustellen. Wenn es in ler Flora Danica (t. 1683 [1821]) heibt: „non raris in ericetis Holsatiae, Lauenburgiae". $\therefore$ ist das eine nur für einen lieinen Teil des Gebiets (den Sachsenwald) die Häufigkeit treffende Bemerkung, doch paßt ,in ericetis" nicht.

1. Untere Blattscheiden kahl:

\section{Formen.}

f. typica nov. f. - Häufigste Form.

Dazu:

f. brachyclada Torges Bot. Ver. Thür. N. F. VIII. 14 (1895). Rispenäste kurz bis sehr kurz, vom Grunde mit Ährchen dicht besetzt, nicht länger oder oft kürzer als die Stengelglieder.

Lauenburg: am Schmalsee bei Mölln 1905!!. Flensburg: Wallsbüll (Prahl!).

- Untere Blattscheiden rückwärts rauhhaarig:

f. hirta P. Junge in Allg. Bot. Zeitschr. XIV. 139 (1908). — So seltener.

Lauenburg: im Sachsenwald am Süsterbek 1906 und im Gehege „Witzhaver Berg" 1912!!.

Calamagrostis varia Host Gram. Austr. IV. 27 t. 47 (1809) kommt nicht vor. Arundo varia Hornemann (Videnskab. Selsk. phys. Skr. I. Deel I. Haefte 194 [1821]) ist mit Arundo varia Schrader $=$ C. varia Host nicht identisch, sondern synonym mit C. andinacea Roth.

\section{$\times(\mathrm{sp} .16 \times 19)$. Calamagrostis Ianceolata $\times$ arundinacea $=$ C. Hart-}

maniana Fries Summa Veget. I. 241 (1846).

4. Grundachse mit zahlreichen, verlängerten Ausläufern. Stengel 0,7-1,5 m hoch, glatt, zuweilen oberwärts ästig. Blattscheiden glatt oder seltener sehr schwach rauh. Blattfläche (1-)2-6 mm breit, beiderseits rauh. Blatthäutchen $1-3 \mathrm{~mm}$ lang, stumpf. Rispe bis $20 \mathrm{~cm}$ lang, mit meistens $1-3 \mathrm{~cm}$ langen. nach der Blüte aufwirts anliegenden Ästen. ت̈hrehen am Rande öfter violett überlanfen. $4-5 \mathrm{~mm}$ lang. Hüllspelzen etwas ungleich, lanzettlich, spitz. Deckspelze etwa $3 \mathrm{~mm}$ lang, mit gerader, die spelze an Lünge überragender oder hinter ilnr zurückbleibender, rücken- oder seltener endstandiger Granne. Hatre der Ährchenachse 1.5-3 mm lang, halb so lang bis sehr wenig länger als die Deckspelze. Blüte Juni bis August.

Selten. - Lauenburg: am Schmalsee bei MIölln (Friedrich 1894!, 1895!. Prahl 1896!) 1904!!: im Sachsenwald am Rande der Benekenriede nach dem Gehege Kammerbekshorst 1904!!. 
Den Mrölner Standort erwähnt Torges (Bot. Ver. Thür. N. F. VII. 23 [1894]).

\section{Formen.}

Die Kreuzung zerfällt:

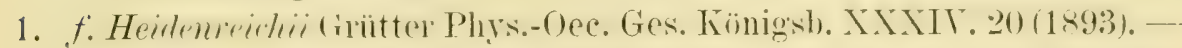
Granne aus etwa ${ }^{3 / 4-S p e l z e n h o ̈ h e ~ e n d s t a ̈ n d i g ; ~ H a a r e ~ d e r ~ A ̈ h r c h e n-~}$ achse etwa so lang wie die Deckspelze. - C. lanceolata nahestehend. Mölln (Friedrich!, Prahl!)!!.

2. f. intermedia Friedrich Fl. v. Lübeck Nachtr. 8 (1900). - Granne aus halber Höhe der Deckspelze rïckenständig; Hare der Ährehenachse etwa von $3 / 4$-Spelzenlänge.

Mölln (Friedrich).

3. f. superamdinacea Torges Bot. Ver. Thür. N. F. VII. 23 (1895). Granne unter der Mitte der Deckspelze entspringend; Haare der

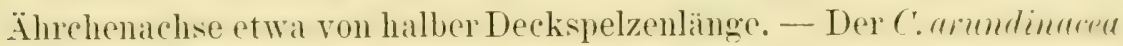
nahestehend.

Mölln (Friedrich!) und Sachsenwald!!.

Bei jeder dieser Formen kann auftreten:

f. puberula Torges Allg. Bot. Zeitschr. VIII. 184 (1902). - Untere Blattscheiden rïckwärts rauhhaarig.

Lauenburg: am Schmalsee bei Mölln (Prahl 1896!).

f. pallida Torges a. a. O. - Hüllspelzen bleichgrün.

Lauenburg: Mölln, in Übergangsformen!!.

f. laxa Torges a. a. O. VIII. 184 (1902). - Lockerrispig; Rispenäste wenigährig, schlaff, überhängend.

Lauenburg: Mölln, in Annäherungsformen!!.

68. (s1). 20). Calamagrostis epigeios Roth Tent. Fl. Germ. I. 34 (1788).

4. Grundachse mit langen Ausläufern. Stengel $(0,4-) 0,6-1,5 \mathrm{~m}$ hoch, muter der Rispe rauh, sonst glatt, starl anfrecht. Blattscheiden glatt oder am oberen Ende schwach rauh. Blattfläche $2-7 \mathrm{~mm}$ breit, zuweilen gefialtet oder eingerollt. Blatthäutchen breit. gestutzt, bis $9 \mathrm{~mm}$ lang. Rispe 10-:01)-301 (m lang. nit aufwirts anliegenden oder wenigr abstehenden Ästen. Ihrehen schmal lamzettlich. 5-6 mm lamg: Hüllspelzen fast gleich lang, spitz ansqezogen. mit rauhem Tiel. Theckspelze bis $3 \mathrm{~mm}$ lang, aus der Mitte oder darunter bis $3 \mathrm{~mm}$ lang begrannt. Granne gerade. Haare der Ährchenachse etwa 5 mm lang. Blüte Juni bis August.

An trockenen Orten auf meistens sandigem Boden an Ufern, an

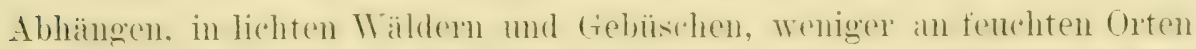
doch zureilen sollst in simmptent. durch das tiebiet meist häufig. doch 


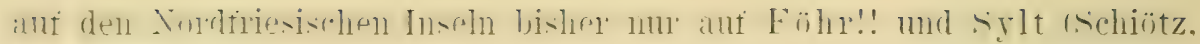
Burcheriatil.

Als Armudo epigeios nemnt Weber die Art (Prim. Fl. Hols. 10 [1780]).

\section{Formen.}

1. Hüllspelzen violett oder rötlich überlaufen:

f. gemina nor. f. - Granne in der Regel kürzer als die Hüllspelzen, bis zu \%/3 ihrer Höhe reichend. - Häufigste Form. Dazu:

f. macrathern Prahl Mitt. Gatt. Calamagr. 7 (1902). Granne aus der Mlitte des Deckspelzenrückens entspringend, die Hüllspelzen an Länge erreichend und zuweilen selbst überragend.

O ldenburg: Dünen der Brök bei Putlos Nolte 183t! z. T., 1838!). - In der Nähe des Gebiets z. B. bei Lübeck: Rosenhagen (Häcker!) und Ra tzeburg: zwischen Römnitz und Kalkhïtte (Prahl 1902!).

f. setifolia nor. f. - Pflanze nur 0,4 m hoch; Blätter (die Grundblätter nicht röllig) borstlich eingerollt.

Eckernförde (J. A. Schmidt 1867!).

f. elonguta Döll Rhein. Fl. 112 (1843). - Rispe deutlich unterbrochen. - In Cbergängen mehrfach beobachtet.

f. densiftorn Ledeb. Fl. Altaica I. 87 (1829). - Rispe sehr dicht. schmal, daher ährenrispig.

Hamburg: zwischen Geesthacht und Krümmel (Zimpel 1893!), Geesthacht (J. A. Schmidt!).

- Hüllspelzen töllig grün; Pflanze graugrün:

f'. Reichentucherene Grec. Fl. Roman. 607 (1898). - An schattigen Orten zerstrent.

Lauenburg: bei Mölln am Schmalsee (Friedrich!) und am Lütaner See (Friedrich!; Tachträge p. 8), Sachsentrald!!. Hamburg (Sonder): Besenhorst (Kausch), Horn (Zimpel!). Pinneberg: Flotbek!!. Lübeck: Falkenhusen (Nolte!), Herrenfähre (Häcker!). Eutin: Kl. Timmendorf (Prahl!), an Cgleisee (Nolte!). Segeberg: Geschendorf (J. Schmidt!). Kiel: Viehburger Wald (Prahl). Angeln: Falshöft!!. Flensburg: Gravenstein (Hansen!). A penrade: Warnitzwig!!. Eine bei uns bisher nicht beobachtete Unterform ist f. Huebneriana Rehb. Fl. Germ. excurs. 27 (1830) mit kurzer, die Deckspelze nicht überragender Granne. - Ubergang in den Typus ist 


\section{f. intermedia Grec. a. a. 0. 607 (1898). - Hiülspelzen mit schmalem violetten Saum. \\ Durch das Gebiet zerstrent.}

Calamagrostis pseudophragmites Baumgarten Enum. Pl. 'Transsilv. III. 211 (1816) fehlt im Gebiete, trotzdem sie mehrfach angegeben worden ist. Die Notizen gehen meistens auf Nolte zurïck, der auch Veranlassung zu der ältesten Nennung bei Hornemann (Vid. Selsk. phys. Skr. I. Deel I. Haefte 194 [1821]) für Lauenburg (ohne Autornamen!) gegeben hat. Diese Notiz für Lauenburg bezieht sich auf denselben Ort, den Nolte später selbst nennt (Nov. Fl. Hols. 11 [1826]): Falkenhusen bei Lübeck. Die als Arundo pseudophragmites Haller fil. aufgeführten Exemplare stammen von 1821! und 1822!; sie gehïren zu C. lanceolata (so auch Prahl Krit. Fl. 249 [1890]). Auch 1824 noch sammelte Nolte Material am gleichen Orte (vgl. Torges Bot. Ver. Thür. N. F. XI. 78/79 [1897]), und zwar wieder C. lanceolata. Reichenbach scheint bereits den Irrtum Noltes gekannt zu haben, denn obgleich er sonst Noltes bemerkenswerte Angaben aufnahm, fehlt C. pseudophragmites (= Arundo littorea Schrader, C. littorea P. B. und auch [später] DC.) unter seinen Fundorten (FI. Germ. excurs. 26 [1830]), während (p. 27) vermerkt wird, daß die Art oft mit C. lanceolata verwechselt werde. Unter C.psendophragmites fehlt Noltes Standort auch bei G. F. W. Meyer, der Holsteins seltene Pflanzen mit aufzählt, erscheint aber bei ihm unter Arundo calamagrostis L. b. nutans Rehb. (Fl. Germ. excurs, $26[\mathbf{1 8 3 0 ]})=$ C. villosa Mutel. Damit steht im Zusammenhange, daß einige spätere Autoren die Angaben der C. villosa aus dem Gebiete zu C. pseudophragmites stellten. Das geschah besonders durch Knuth (FI. v. Schlesw.-Holst. 754 [1887] und Schulfl. v. Schlesw.-Holst. 364 [1887]). Daß C. pseudophragmites bei Borchmann (Fl. v. Holst. 71 [1856]), Laban (Fl. v. Holst. 224 [1866]), Lienau (Fl. Fürstent. Lüibeck 7 [1863]) und Langmann (Fl. v. Necklen̈b. etc. noch 3. Aufl. 271 [1871]) wiedererscheint, kann nicht weiter wundernehmen; mehr schon, daß H. G. Reichenbach ihre Ungenauigkeit nicht kannte und sie wiederholte (Vorlesungsverz. Hamb. 20 [1881]). C. pseudophragmites "Schrader", von Hennings (Nat. Ver. Schlesw.-Holst. II. 1. 145 [1876]) für Hohenwestedt aufgeführt, ist wohl Synonym von C. lanceolata. Zu dieser Art zählt auch C. pseudophragmites Haller fil. desselben Autors vom Schulensee bei Kiel (Nolte) (Nat. Ver. Schlesw.-Holst. II. 1. 202 [1876]).

\section{$\times$ (s). $19 \times 20$. Calamagrostis arundinacea $\times$ epigeios $=$ C. acutiflora}

Reichenbach Fl. Germ. excurs. 26 (1830).

4. Grundachse mit kurzen Ausläufern und dichtstehenden Halmen. Stengel $0.8-1,6 \mathrm{~m}$ lang. aufrecht, unter der Rispe ziemlich rauh. Blattscheiden glatt oder affer schwach rauh. Blattlärhe $20-6 \mathrm{~mm}$ breit. flach, lebhaft grïn. Blatthäutchen 1-3 mm lang. stmmptlich. zerrissen. Rispe 10-20 cm lang. nach der Blüte schmal zusammengezogen, mit bis 5 cm langen Ästen. Ährchen 5 mm lang, schmal lanzettlich. spitz. Deckspelze etwat $3 \mathrm{~mm}$ ling. im unteren Drittel des spelzenrückens inseriert, gerade oder sehwach gekniet, bis $3 \mathrm{~mm}$ länger als die Deckspelze. öfter die Hüllspelzen überragend. Haare der Ährehenachse meistens kürzer als die Deckspelze, 2-3,5 mm lang.

Im sïdöstlichen Gebiet sehr selten. 
Lauenburg: im Sachsenwald am Abhange der "Schwarzen Aue" zwischen Kupfermühle und Stangenteich 1904, 1906, 1912!!.

Die Angabe der Kreuzung für Lauenburg (A. u. Gr. Syn. II. 1. 219 [1899]) beruht nach Prahl (Mitt. Gatt. Calumagrostis 8 [1902]) auf einem Irrtum.

\section{$\because$ (:1) $16:$ 20). Calamagrostis lanceolata $<$ epigeios $=$ C. Neumaniana Torges But. \\ Ver. Thür. X. F. XVIr. 93 (1902).}

Eine möglicherweise hierher gehörige Form sammelte Prahl in Lauenburg: am Schmalsee bei Mölln 1895!. Mir war es ummöglich, den Einfluß von C. lanceolata zu erkennen. Thüringische Pflanzen (leg. Reinecke!) weichen jedenfalls bedeutend ab (rgrl. Prahl Nitt. Gatt. Calamayrostis 10. [1902] und Torges a. a. 0.).

69. (s). 21). Calamagrostis arenaria Roth Tent. Fl. (term. I. $34(178 \pi)$.

4. Grundachse dick, reich rerzweigt. Stengel dicht gestellt, anfrecht, 0,5-1 m hoch. Blattscheiden glatt oder die unteren schwach rauh, derb. Blattfläche eingerollt, 1-3 mm dick, glatt, oberseits auf den Nerven behaart. Blatthäutehen aus breitem (irunde bis -..5 cm lang. allmählich verschmälert. zweispitzig: spitzen lis 7 mm lang: Rispe dicht, ähremrispig. 6-15 cm lang, heiderseits rerschmälert. Ährehen $1 \mathrm{~cm}$ lang. Hüllspelzen schmal lanzettlich. untere eimnervig. obere dreinervig. fast gleich lang. Deckspelze fünfnervig. lanzettlich. ans kurz zweizähniger spitze bis $1 \mathrm{~mm}$ lang begramnt. mit schwach rauhem fiel. bis $8.5 \mathrm{~mm}$ lang, wenig länger als die Vorspelze. Haare der Ährchenachse bis $3 \mathrm{~mm}$ lang.

Auf Dünen, auf Sandfeldern und an sandigen Abhängen an der Nord- und Ostsee sowie an der Elbe häufig oder nicht selten, sonst zerstrent; an vielen Stellen zur Dünenbefestigung gepflanzt.

Weber nennt die Art (Prim. Fl. Hols. 11 [1780]) als Anundo arenaria.

\section{Mißbildungen.}

f. bracteata nor. f. - Ährenrispe mit laubigem Tragblatt. - Stormarn: Ladenbek!! Kiel: Schönberger Strand (A. Christiansen!).

\section{‥1. $20 \times 21$. Calamagrostis epigeios $\times$ arenaria $=$ C. haltica Hartman}

Handbok Skand. Fl. 2. Uppl. 20 (1832).

4. Grundachse stark verzweigt. Stengel zahlreich, aufrecht. Blattweheiden glatt oder sehr schwach rauh. Blatthiache eingerollt, glatt, oberseits kurhatarig. Blatthäutchen bis $1,5 \mathrm{~cm}$ lang. allmählich rerschmälert, zweispitzig. Rispe 10-25 cm lang. deutlich gelappt. mit längeren Ästen. Ahrehen !-10 mm lang. Hüllspelzen meistens tast gleich lang; am Rande 
violett ïborlanfen, untere rimmervig, obere droinervig, oberwaits am Kiral ranh. Deckspelze 7 - 8 mm lang, undeutlich fïnfuervig, spitz, mubegramut oder dreispitzig laus binsehnitt mit 0,5 mm langer (rimme). Torspelze so

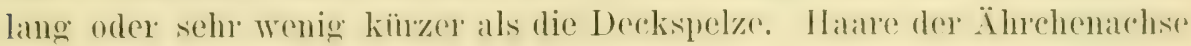
bis $5 \mathrm{~mm}$ lang.

Auf. Dünen der Ostsee und (seltener) der Nordsee.

Ostsee: von 'l'atremünde bis Hadersleben nicht selten bis zerstrent. nördlich der Flenshurger Föhrde seltener; an der Lntertrare aufwäts

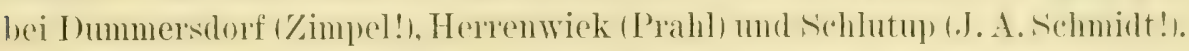

Nordsee: Helgoland: auf der Düne (Knnth!; wahrscheinlich zur

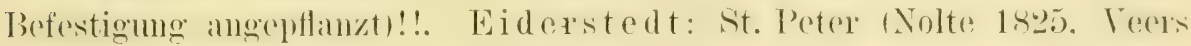
1833!, Hansen 1845!, v. Nüller 1847!) 1905!!. A mxunı (v. FischerBenzon. J. Sehmidt!): W'ittdün (Prahl!) und Norddorf!!. Föhr (r. FischorBenzon). Röm: Lakolk (Jaap).

Nolte fand die Pflanze im Gebiete auf bei Travemünde (1820!, 1821 !). śrine oder Flügges?) Beobatchtung veröffentlichte Hornemam, und zwal in einem Jahre an drei Stellen (Oec. Plantel. 3. Udg. I. 86, Flora Danica t. 1684 in Bd. X. Heft 29 und Tid. Selsk. phys. Skr. I. Deel I. Haefte 192 [1821]).

\section{Formen.}

1. Rispe dichter; Ährchen etwa $10 \mathrm{~mm}$ lang; Hüllspelzen fast gleich: f. subarenaria Prahl Krit. Fl. 251 (1890). - So fast stets.

- Rispe stäker gelappt; Ahrchen 18,5-19 mm lang; Hüllspelzen 1 m etwa $1 \mathrm{~mm}$ verschieden lang:

$f$. subepigeios Prahl a. a. 0. 251 (1890). - So seltener.

Kiel: Schönberger Strand (A. Christiansen!). - Einen Übergang zu dieser Form bilden nach Prahl (Krit. Fl. 251) Noltesche Exemplare aus Eiderstedt, auf welche sich vielleicht Röpers Bemerkung (Zur Fl. Mecklenb. 192 [1843]) bezieht, nach welcher Nolte ihm (Röper) mitgeteilt hat, für die Hybridität der Pflanze spreche es, daß sie in Holstein in zwei Formen vorkomme. Tch habe die Pflanze in dieser Form nicht beobachtet.

\section{Gattung.}

\section{Holcus.}

L. Gen. plant. ed. 5. 469 (1754).

1. Granne die Hüllspelzen nicht überragend .................... lanatus.

- Grame die Hüllspelzen überragend .H. mollis. 
70. (. 2). 22). Holcus Ianatus L. Spec. plant. ed. 1. 1048 (1753).

4. Stengel zahlreich, aufrecht oder aufsteigend, $0,4-1 \mathrm{~m}$ hoch; glatt. mit dicht kurzbehaarten linoten. Blattscheiden muterwärts stärker, oberwärts schwächer dicht rückwärts weichharig, etwas aufgeblasen. Blattläche $4-10 \mathrm{~mm}$ breit, beiderseits zerstrent kurhaarig. Blatthäntchen etwa $2 \mathrm{~mm}$ lang, gestutzt. Rispe $4-12 \mathrm{~cm}$ lang, locker, mit dicht kurzhaarigen Ästen. Ährchen 3,5̃-5 mm lang. meist zweiblütig. Hüllspelzen fast gleich lang, gekielt, am Rüchen hehaart, untere eimnervig, obere dreinervig, breiter. Declispelzen 12-25 mm lang. glänzend, die der oberen (männlichen) Blüte mit lis əo mm langer, gekrümmter, muter der Spitze stehender Granne. Blüte Juni bis August.

An trockenen, seltener feuchten, Orten durch das ganze Gebiet häufig (auch auf den Nordseeinseln).

Zuerst erwähnt von Weber (Prim. Fl. Hols. 72 [1780]).

\section{Formen.}

1. Hüllspelzen hellrötlich oder lila ïberlaufen:

f. coloratus Reichenbach Ic. Fl. Germ. et Helv. I. 15 (1834). Häufig.

- Hüllspelzen grïnlich oder grünlichgelb:

f. albovirens Reichenbach a. a. O. (1834).

Zerstreut. Z. B. beobachtet Lanenburg: bei Dermin, in der ..Aue" und bei Krümmel!!. Stormarn: Reinfeld (J. A. Schmidt!), Fischbek!!. Hamburg: Horn (J. A. Schmidt!), Farmsener Moor!!, Geesthacht!!. Pinneberg: Schulau!! Föhr (Schiötz als f. argenteus Lange). Sylt: Eidumer Vogelkoje!!. Röm: Kirkeby (Raunkiaer). - Die Formen mit grünlichgelben Ährchen nähern sich der f. flarescens Waisbecker.

\section{1. (sp. 23). Holcus mollis L. Systema ed. 10. 1305 (1759).}

7. Stengel entfernt ans kriechender Grundachse, aufrecht, $0,2-0,8 \mathrm{~m}$ hoch, glatt, an den Kinoten meistens behart. Blattscheiden kahl oder teilweise oder sämtlich behaart, selten erweitert. Blattfläche $4-8 \mathrm{~mm}$ breit, beiderseits schwach behaart, am Rande oft etwas rauh. Rispe 3- s cm lang. mit weniger abstehenden Ästen. Äste sehr schwach behaart. Ährehen 5-6 mm lang. Hüllspelzen gelblich, spitz, zerstrent schwach behart, die untere, eimmervige etwas kürzer als dic obere, dreinervige. Deckipelzen 2-2,5 mm lang. grünlich, die der oberen Blüte aus dem oberen Teile des Rückens mit etwa 4 mm langer, die Hüllspelzen bis $2 \mathrm{~mm}$ überragender Granne. Blüte Juni bis August. 
An Weg- und Ackerrändern, an Ablängen, in Gebüschen und W nicht selten bis zerstrent, doch auf den Nordfriesischen Inseln nur auf Fölr: Wyk (Ramkiaer), Amrum (Raunkiaer): Nebel und Satteldüne!!,

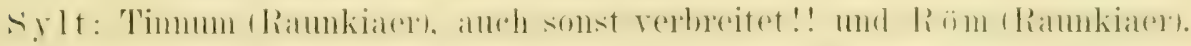

Die erste Erwähnumg findet die Pflanze bei Weber (Prim. Fl. Hols. $72[1780])$.

\section{Formen.}

Nicht beobachtet.

\section{Gattung.}

\section{Avena.}

L. (ien. plant. ed. 5. 34 (1754).

1. Ährchen mit unterer männlicher und oberer zwitteriger Blüte....... elatior.

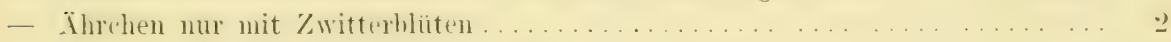

๖. Pflanze 24; Hüllspelzen höchstens fünfnervig................

- Ptlanze $\odot$; Hüllspelzen sieben- oder mehrnervig ................ $t$

:. Pflanze lockerrasig; Blattscheiden fast stets dicht behaart; Blattspreite flach.

A. pubescens.

- Pflanze dichtrasig; Blattscheiden kahl; Blattfläche eingerollt.....A. pratensis.

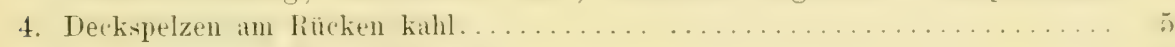

- Deckspelzen am Rücken behaart............................

5. Seitenspitzen der Deckspelze gramenartig lang ausgezogen ........ strigosa.

- Seitenspitzen der Deckspelze nicht grannenartig ................ is

6. Hüllspelzen höchstens die Bliiten an Länge erreichend, sie nicht überragend.. †

- Hüllspelzen die Blüten deutlich überragend ..................

7. Ährchen bis $3 \mathrm{~cm}$ lang, drei bis sechsblütig; Deckspelze oberwärts rerschmälert. A. nuda.

- ت̈hrchen bis $1,5 \mathrm{~cm}$ lang, zweiblütig; Deckspelze oberwärts rerbreitert.

A. brevis.

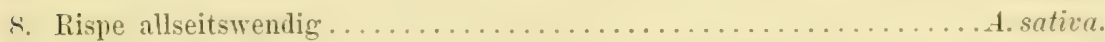

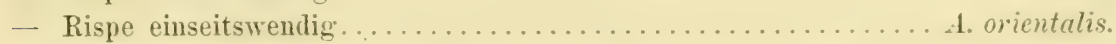

(1. Seitenspitzen der Deckspelze grannenartig lang ausgezogen........ d. barbata.

- Seitenspitzen der Deckspelze spitz . . . . . . . . . . . . . . . . . 10

10. Ahrchen drei- bis sechsblütig; Blüten zusammen ausfallend ..........steritis.

- Ährchen zwei- bis dreiblutig; Blüten einzeln ausfallend ............ fatua.

72. (sp. 24). Avena elatior L. Spec. plant. ed. 1. 79 (1753).

4. Stengel aufrecht oder aufsteigend, $(0,3-) 0,6{ }^{\circ}-1(-1,6) \mathrm{m}$ hoch, glatt. Blattscheiden glatt oder oberwärts schwach rauh. Blattfläche 3-10 mm breit, unterseits stärker, oberseits schwächer rauh. Blatthäutchen kurz, gestutzt. Rispe $6-15(-20) \mathrm{cm}$ lang, ziemlich kurzästig, schmal. Ährchen bis $11 \mathrm{~mm}$ lang. Hüllspelzen häutig, ungleich, die 


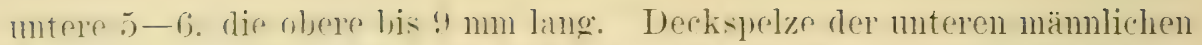
Blïte (f)-k-11 mm lang. mit tief (zuweilen fast grundständig) rückenstandiger lirame: Granne gekniet, unten gedreht. $8-15-191$ mm lang. Deckspelze der Zwitterblïte 7-11 mm lang, mit kurzer, im Spitzen-

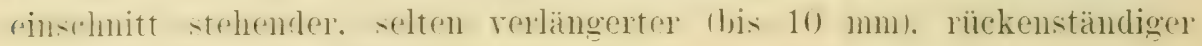
Gramne. Blüte Juni bis Juli, öfter einzeln noch später.

Auf Wiesen und Triften, an Wegrändern und Feldrainen, an Ab-

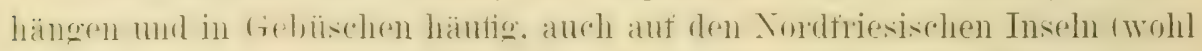
mit Grassaat eingeführt) Föhr: rerbreitet 1910!!, Sylt: Tinnum und

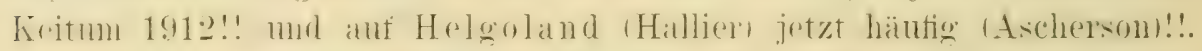

Weber nemnt die Art (Prim. Fl. Hols. 72 [1780]) als Holcus avenacens (nach Scopoli).

Für Föhr gibt schon Knnuth die Pflanze an (Nat. Ver. Schlesw.Holst. IX. 1. 96 [1891]), wiederholt den Termerk aber später nicht (Fl. Nordfries. Inseln $144[1895]$ ).

\section{Formen.}

1. Stengel an Scheiden und Knoten kahl............... 2

- Stengel an den unteren Scheiden und Knoten behaart:

f. subhirsuta Ascherson Fl. Brandenb. I. 826 (1864).

Ziemlich selten. - Lüibeck:Pöppendorfer'T'rave-Ufer!!. Neustadt: Brodau (sehr charakteristisch)!!. Kiel mehrfach (A. Christiansen!). -- Ubergangsformen treten auf, bei denen nur die Knoten behaart sind, so z. B. auf Helgoland!!. Dazı gehört die Abweichung, welche Sickmann (Enumeratio 10 [1836]) als $\beta$. nodis villosis bezeichnet.

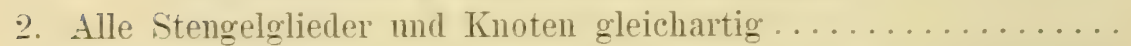

- Untere (zwei bis drei) Stengelglieder im mteren Teile (am Knoten) knollig rerdickt:

f. tuberosa Ascherson a. a. O. I. 826 (1864).

Selten. - Hamburg (Sonder, kombiniert mit f. subhirsutc). Helgoland: beim Leuchtturm (Hallier als f. bulbosum) und beim Pulvermagazin (Brody nach Knuth Allg. Bot. Zeit. 109 [1898]). -- Hin und wieder treten Annäherungsformen auf (! und !!).

3. Deckspelze der oberen Blüte gramnenlos oder kurz begrannt... 4

- Deckspelzen beider Blïten mit nach Form und Größe wenig rerschiedenen, das Ährchen weit überragenden Gramen:

f. biaristate Petermann Fl. Bienitz 14 (1841). - So zerstreut bei recht wechselnder Ausbildung der Gramen (vgl. Bochkoltz Bot. Ver. Brandenb. III/IV. 288/89 [1862]).

Z. B. beobachtet: Lauenburg: \[ölh, Alt-Mölln, 
Kastorf, Friedrichsruh!!. Ha mburg: Wilhelmsburg(C.'Timm), Warwisch und Curslack!!. Veddel (.J. Schmidt!). Kuhwärder (Beyle!), vor dem Dammitor (Laban 1865!). Stormarn: Alt-Rahlstedt und Bargteheide!! Pinneberg: Flottbek (Kausch!). Lïbeck: Zwischen Burg- und Hüxtertor (K. Bumester!). Neustadt: Pelzerhaken!!. Oldenburg: Neu-'Teschendorf!!. Kiel mehrfach (A. Christiansen!). Eckernförde: Sehestedt und Grasholz (A. Christiansen!). Hadersleben: Gravenshoved!!. Sylt: Keitum!! - Ubergänge treten hier und da auf (dazu f: subbiaristata Jahrb. Hamb. Wiss. Anst. XXII. [1905]).

4. Deckspelze der unteren Blïte kahl.....................

- Deckspelze der unteren Blïte behart:

f. pilifera A. u. Gr. Syn. Mitt.-Europ. Fl. II. 1. 230 (1899).

Zerstreut; besonders an trockenen, sandigen Orten. -

La uenburg: in der ,Ane*!!. Lübeck: Herrenwiek und Pöppendorf!!. Lütjenburg: Hohwacht!!. Ki lel: Söhren, Achterwehr und am Langensee (A. Christiansen!). Angeln: von Glücksburg bis Westerholz mehrfach!!. Sonderburg: Sandacker und Gravenstein!!.

5. Ährchen zweiblütig:

f. vulgaris Eries Nor. Fl. Suec. Mant. 3 p. 4 (1842). Häufig:

Daz11:

f.brachyclada Reinecke (in sched.). - Rispenäste verkürzt, besonders die unteren oft viel kürzer als die Stengelglieder, daher Rispe unterbrochen. - Lübeck: Blankensee (Friedrich!). Fehmarn: zwischen Petersdorf und Lemkendorf!!. Kiel: Stein, Nönkeberg und Rotenhahn (A. Christiansen!).

f. microstachya nor. f. - Ährchen nur 5-6 mm lang. -

Kiel: Stein! und Barsbeck! und Eckeruförde:

Schnaap!(A. Christiansen 1912). F lensbur' g* Föhrdeabhang bei der Kupfermühlenhölzung 1904!! (kombiniert mit f. glanca Beck Fl. Nied.-Osterr. 72 [1890]).

- Ährchen (zwei- bis) dreiblütig, zwitterig:

f. hermaphorlita Lange Haandb. Danske Fl. 3. Udg. 83 (186t).

'Typisch nicht beobachtet; in sehr angenäherter Form mit stets drei Blïten im Ährehen, die aber' $z$.'T. verkiummerte Fruchtknoten haben: Ki el: Mönkeberg! und R ends burg: Ostenfeld! (A. Christiansen 1912). 


\section{Mißbildungen.}

f. m. imitira nor. f. - Stengel aus den oberen Knnten mit rispentragenden Ästen. - Kiel: Neustein (A. Christiansen!)

73. (adr. 42). Avena sativa L. Spec. plant. ed. 1. 79 (1753).

$\odot$. Stengel aufrecht, am Grunde in der Regel verzweigt, $0,4-1,2 \mathrm{~m}$ linch. glatt. Blattscheiden kahl oder selten zerstreut behaart, glatt. Blattfläche 5-12(-17) mm breit, rauh. Blatthäutchen kurz, breit, grezähelt. Rispe mit allseitig abstehenden Ästen. Ährchen $2-31-3,81 \mathrm{~cm}$ lang. meistens zweiblütig. Hüllspelzen bis 8.8 cm lang. die untere etwa $2 \mathrm{~mm}$ hürzer als die obere untere sieben- bis neumervig, obere acht- bis elfnervig. Deckspelze glatt. glänzend. oberwärts grün, grannenlos oder begrannt. Grame gerade, fein. die Hüllspelzen ïherragend. Älırchenalchse nur unter der muteren Blüte kurzhaarig. Blüte Juni bis August. einzeln später.

Im ganzen feliete gebaut und an Wegen. anf Ackerland und fartenboden verwildert, auf Schutt rerschleppt; häufig.

\section{Formen.}

1. Deckspelze (wenigstens der untersten Blïte) begrannt:

$f \circ$ aristata Krause Getreide Heft 7. $6(1835 / 7)$. - So häufiger. Dazul :

f. trisperma Schübeler Diss. bot. 8 (1825). - Ährchen dreiblütig. - Um Hamburg mehrfach auf Schutt (zuerst C. Timm)!!.

- Deckspelze grannenlos:

f. muticr Kranse a. a. O. $6(1835 / 7)$. — So seltener. Dazu:

f. longighumis nor. f. - Hüllspelzen 3,5-3,8 cm lang, 1,5mal so lang wie die Deckspelze der unteren Blïte. - Hamburg: Fuhlsbüttel 1912!!.

f. hivta nov. f. - Untere Blattscheiden rückwärts rauhhaarig. - H a mburg: Fuhlsbüttel (J. Schmidt)!!.

74. (adr. 43). Avena orientalis Schreber spic. Fl. Lips. 52 (1771).

Der vorigen Art ähnlich, aber: Stengel bis $1 \mathrm{~m}$ hoch. Rispe mit einseitig gestellten, fast aufrechten Ästen, schmal.

Auf schut bei Hamburg und Lübeck nicht selten, sonst zerstrent und oft einzeln; zuweilen untersathafer und im Mengfutter. selten für sich gebaut.

Formen.

Nicht beobachtet. 


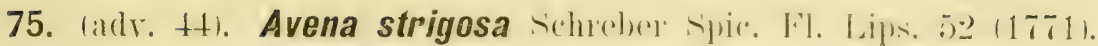

$\odot$. Stengel aufrecht, an Grunde verzweigt, 0,3-0,8 m hoch, glatt.

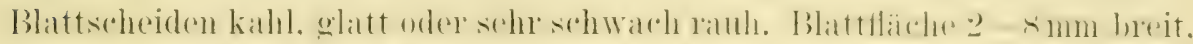

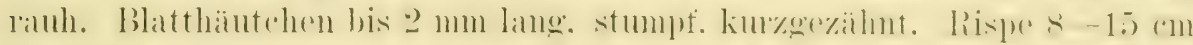

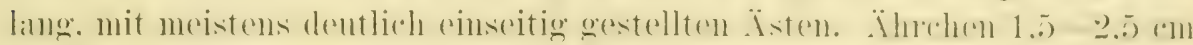
lang, schmal. Hüllspelzen etwa $2 \mathrm{~mm}$ verschieden lang, untere sieben-. obere nemnervig. Deckspelze glänzend, oberwärts rauh, mit rïckenständiger, schwach geknieter, unterwärts gedrehter Granne und zwei

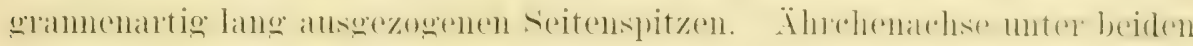
Blüten kurzharig. Blüte Juni bis Angust, einzeln später.

Auf Schutt, an Wegen und auf Äckern hin und wieder verschleppt, doch viel seltener als A. sutiva; auf Sandboden gebant.

\section{Formen.}

Nicht beobachtet.

76. (adv. 45). Avena Jrevis Roth Botan. Abhandl. 42 (1787).

$\odot$. Stengel aufrecht, unten verzweigt, 0,5-1 m hoch, glatt. Blattscheiden glatt. Blattfäche $4^{--8} \mathrm{~mm}$ breit, lauh. Blatthäutchen kurz. stumpt, gezälnnt. Rispe $5-12 \mathrm{~cm}$ lang, mit einseitig gestellten Ästen. meistens locker. Ährchen 12-15 mm lang. Hüllspelzen breit, untere sieben- bis neumnervig, $12 \mathrm{~mm}$ lang; obere acht- bis elfnerrig. bis $15 \mathrm{~mm}$

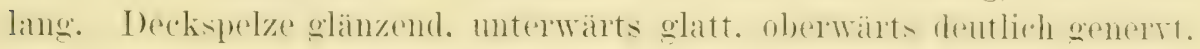
zweispitzig;, mit rückenständiger, geknieter Granne, über der Mitte am breitesten, rauh. Ährchenachse unter beiden Blïten behaart oder unter der unteren kahl. Blüte Juni bis August.

Auf Schutt und Haferfeldern selten.

Ha mburg: Winterhuder Bruch (J. Schmidt 1891!), Wandsbek (Sonder nach Fl. Hamb. 47 [1851], Klatt 1859!). Husum: Ahrenviöl (unter A. strigosa) (A. Christiansen 1912!).

Linuth bezeichnet (Schuifl. v. Schlesw.-Holst. 365 [1S8б]) die Art als: ..Bei uns selten gebaut, hier und da verw." (verwildert), auf Grund welcher Beobachtungen, ist nicht zu ersehen.

77. (adv. 46). Avena nuda L. Amoen. Acad. IIT. 410 (1756).

$\odot$. Stengel aufrecht, $0,4-0,9 \mathrm{~m}$ lioch, glatt. Blattscheiden glatt. kahl. Blattfläche $3-8$ mm breit, rauh. Blatthäutchen kurz, gezähnelt.

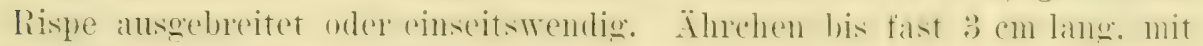

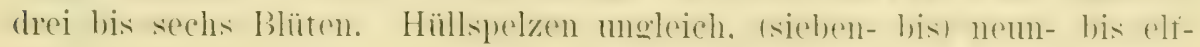


nervig. Deckspelze zweispitzig. gramenlos oder begrannt. deutlich nervig. Ährchenachse gestreckt, dünn, kahl. Blüte Juni bis August.

Bisher nur Hamburg: unter (ietreide sonder Fl. Hamb. 47 [185̃1]).

Tor Sonder findet die Pflanze durch Hübener (Fl. r. Hamb. 497 [1847]) Erwähnung. Birrimann hat die Pientrkung: Ancrehaut und rerwildert (Fl. r. Holstein 74 [1856]).

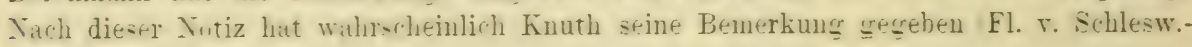
Holst. 760 [1887] und Schulfl. v. Schlesw.-Holst. 366 [1887]).

78. (adv. 47). Avena fatua L. Spec. plant. ed. 1.80 (1753).

$\odot$ (und $\odot \%)$. Stengel aufrecht, am Grunde verzweigt, glatt. Blattscheiden glatt oder schwach rauh. Blatflache :3-10 mm breit, rauh. Blatthäutchen bis $4 \mathrm{~mm}$ langr. spitzlich. gräahnelt. Rispe allseitig ansgetoreitet. Alurchen etwa $12-12.5 \mathrm{~cm}$ lang, meistens dreiblütig. Untere Hüllspelze sieben- his nemmervig. obere Hüllspelze (nem- bis elfnervig. rtwas länger als die untere. beide spitz. Deckspelze bram. lang bramroder weisharig. mit grünen seitenspitzen und aus der Rürkenmitte entsluringender. kraftiger. greknieter und gedrehter frame. im unteren Teile glatt. sonst mit starken Nerren. oberwarts rauh. Ährehenachse langhaarig. bei der Reite zerfallend. daher die Blïten einzeln abfallend. Blïte Juni bis Juli, zuweilen später.

Hin und wieder auf schutt und Baggerland. an Häfen und Bahnhöfen und bei Iühlen. seltener unter Getreide und auf Kartoffeläckern.

Lauenburgr: am Lanenburger Hafen!!.. am Ziegelholz bei Möln (Friedrich 18.4!!. Hamburg I Möblerı: auf den Elbinseln unter Getreide und auf schutt somder. Wollhämmerei am Reiherstieg (Zimpel'ı. Stein-

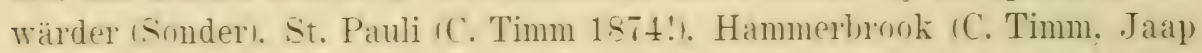

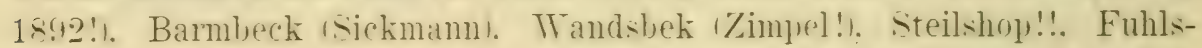
lüttel:.: Tinterhude mehrfach (C. Timm usw. !)!.. Eppendorf (Laban!). Lokstedt ILaban!. Langenfelde IA. Junge!. Bahrenteld!.. Oldenburg i. H.: Heiligenhafen!!. Kiel: am Galgenteich (Prahl 1-8s). Yeustein und Fofhrowh A. Fhristiansen!. Flensburg: Stranderott und Inunkmühle!!. Alsen (Petitı. Tondern: Deezlü̈ll und Kileisese Kong (Jörgensen 184t!). sylt: Keitum!!. Amrum: Yelel!:. Helgoland mehrfach (Hallier usw.)

Die erste Notiz der Pflanze gibt für das Gebiet Weber (Prim. Fl. Hols. $9[1780]$ ).

Eine Reihe ron Bemerkungen gibt zu der schon ron Mößler (Handb. Gewächsk. 147 [1815]) bei Hamburg nachgewiesenen Art Hübener (Fl. F. Hamb. 497 [1847]): „um Schöningstedt, Glinde und in der Harksheide gebaut (?), „häufig auf Getreidefeldern rerwildert". Seine Arbeit war rielleicht die Quelle für Knuths Angabe: - unter der Saat nicht selten" (Fl. r. Schlesw.-Holst. 760 [1887]). Der Flughafer ist durchaus nicht häufig, wenn es auch wahrscheinlich ist. daß man ihn vielfach überseheu hat. Das älteste gesehene Exemplar trägt die Bezeichnung: Itzehoe (J. J. Meser 1817!). Es ist aber sehr fraglich, 
ob J.J. Neyer die Pflanze bei Itzehoe gesammelt hat, ob er nicht vielmehr nur sagen wollte, daß er dieselbe 1817 in Itzehoe für sein Herbar erhalten hatte. Sonder sagt (Fl. Hamb. 48 [1851]), dah A. fatue in nordöstlichen Holstein vorkomme; leider fehlt eine Bezeichung des Fundortes.

\section{Formen.}

f. albescens Sonder in Döll Fl. v. Baden I. 190 (1857). - Haare an Ährchenachse und Deckspelze weiß.

Vereinzelt mit dem 'Typus (! und !!)。

79. (adv. 48). Avena sterilis L. Spee. plant. ed. 2. 118 (1762).

$\odot, \odot$. Stengel unterwärts verzweigt, glatt. Blattscheiden glatt. Blattfläche $4-10 \mathrm{~mm}$ breit, rauh. Blatthäutchen $3 \mathrm{~mm}$ lang, am Rande zerschlitzt. Rispe einseitswendig, mit rauhen, 1-3(-4) Ährchen tragenden Ästen. Ährchen bis etwa 4 cm lang, vier- bis fünf- (bis sechs-blütig. Hüllspelzen spitz, bis $4.2 \mathrm{~mm}$ lang. Die beiden unteren Deckspelzen bis $3 \mathrm{~cm}$ lang, braun. starknervig, lang ausgezogen zweispitzig, in der unteren Hälfte mit langen, gedrehten, gelblichen Haaren, aus der Rückenmitte mit im unteren Teile gedrehter, geknieter, derber, bis $6 \mathrm{~cm}$ langer Grame. Obere Deckspelzen kïrzer, unbegrannt. kahl, nur die dritte ausnahmsweise begrannt. Ährchenachse im unteren Teile behart, zäh, daher sämtliche Blüten zusammen ausfallend. Blüte Juni bis August.

Auf Schutt bei Hamburg selten.

Hamburg: auf der Hoheluft (Kausch 1888!).

\section{$\times$ (adv. $42 \times 47$ ). Avena sativa $\times$ fatua $=$ A. hybrida Petermann Fl. d. Bienitz 13 (1841).}

$\odot$. Stengel aufrecht, $0,3-1,2 \mathrm{~m}$ hoch, glatt. Blattscheiden in der Regel glatt. Blattfliche $4-10 \mathrm{~mm}$ breit, rauh. Rispe ansgebreitet oder zusammengezogen, mit aufrechten oder abstehenden, zuweilen einseits gestellten Ästen. Ährchen 2-2,5 cm lang, meistens zweiblütig. Hüllspelzen wenig ungleich. Deckspelze weiblich bis gelbbrämlich. unten breit, nach oben allmählich schmiller, unten glatt, nur in der oberen Hïlfte mit dentlichen Verven. zerstrent kürger behant bis fast kahl. mit rïckenständiger.

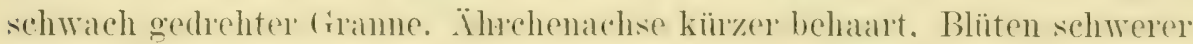
sich tremnend als bei $A$. fatua. Blüte Juli bis August (bis Oktober).

Auf Schutt und Baggerland, zuweilen anch unter Getreide, sehr zerstreut.

H amburg:- Bergedorf!!, Zollenspieker (Kreuzung mit A. orientalis)!!,

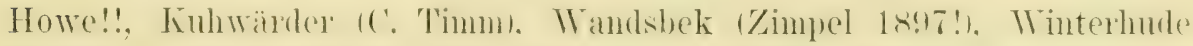

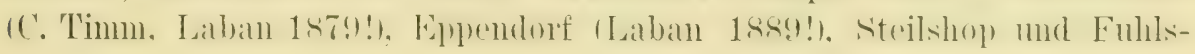


biittel 1912!!, Holstenbrauerei in Altona (J. Schmidt!), Langenfelde (A. Junge!), Bahrenfeld!!. Lübeck: zwischen Burg- und Hüxtertor

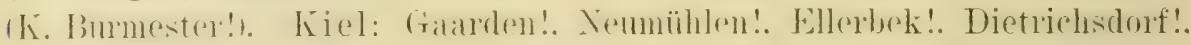
Silberberg bei Wisch!, Kronshagen!, Havighorst! und Rendsburg: Sehestedt! (A. Christiansen). Tondern: Deezbüll (Jörgensen!). - Jedenfalls viel weiter verbreitet.

Die erste Angabe ans dem Gebiet hat Koch (Syn. Fl. Germ. et Helv. ed. 2. II. 917 [18t4]) von 'Tondern.

Die Trennung dieser Pflanze von A. fatua ist ganz sicher nur dann möglich, wenn man alle Avena-Formen mit schwächerer Behaarung, als sie der Typus von A. fatua besitzt und die zugleich breitere Deckspelzen, schwächere Deckspelzennervatur, schwächere Granne und festere Ährchenachse aufweisen, zu der Kreuzung stellt. Übrigens lehren die Tersuche ron Zade, dab A. Iybrida keine Hybride zwischen getremnten Arten ist, sondern nur ein Produkt aus Rassen derselben Art. Die sogenannten (auch vorstehend su aufgeführten) „Arten“ (der Sektion Euarena gehören nach dem Verhalten der A. hybrida bei Kulturversuchen sämtlich zu einer Art, können demnach auch lieine Artenhybriden liefern. Wem eine "Hybride" sich in der Kultur in die "Eltern" aufspaltet, so ist sie ron ihnen nicht wesentlich verschieden und sind auch die Eltem wesentlich gleich, da bei stärker differenzierten alten Arten ein gleiches Verlalten nicht eintritt, auch gar nicht eintreten kamn, da die Samen fehlschlagen.

Im Namen der Kreuzung ist A. sativa als Gesamtart gefabt (A. u. Gr. Syn. Mitt.Europ. Fl. II. 1. 233 [1899]), so das A. orientalis, A. strigosa, A. brevis und A. nuda mit inbegriffen sind. Eine Pflanze von Zollenspieker!! ist zweifellos A. orientalis $\times$ fatua; eine von Husum: Ahrenviö (A. Christiansen!) mir gesandte ,A. strigosa“ mit oberwärts kurzhaariger Deckspelze dürfte A. strigosa $\times$ fatua (ersterer nahestehend) sein. Die Pflanzen der Hamburger Schuttplätze zeigen eine außerordentliche Variabilität.

Die aus unserem Florengebiete gemachten Angaben der A. fatua f. glabrata Peterm. bezichen sich auf die Hybride.

Formen.

f. Thirta nor. f. - Untere Blattscheiden zerstreut rauhhaarig.

Selten. - Hamburg: Steilshop!!. Kiel: Ellerbek!, Kronshagen! und Toflorook! (A. Christiansen).

80. (adr. 49). Avena harhata Brotero Fl. Lusit. I. 108 (1804).

$\odot$ und $\odot$. Stengel $0,5-1 \mathrm{~m}$ hoch, aufrecht. Blätter wie bei A. futuu. Rispe mit schlaffen, einseitig gestellten Ästen. Ährchen etwa 2) ('n lang. meistens zweil)lïtig. Hüllsuelzen wenig ungleich. schwalchnervig. Deckspelzen oberwärts verbreitert, mit deutlichen Nerven, im miteren Teile des Rï̈rkens stark behalart. ans der Mitte mit geknieter.

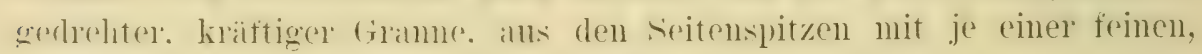
geraden, bis $1 \mathrm{~cm}$ langen Granne. Blute Mai bis Juli.

Auf Schutt bei Hamburg selten.

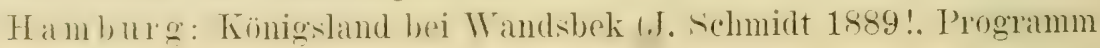


31 [1890]. Ber. Dentsche Bot. Ges. 1X. 126 [1891]), bei der Holstenbrauerei in Altona (J. Schmiclt!).

\section{Formen.}

Nicht beobachtet.

81. (.1). 25). Avena pubescens Huds. FI. Angl. ed. 1. 42 (1762).

4. Stengel ans lockerrasiger Grundachse aufrecht, $0,2-1 \mathrm{~m}$ hoch, glatt, kahl. Blattscheiden im miteren Stengelteile fast stets behaart, die oberen zuweilen kahl. Blattliache unterseits stäker, oberseits schwächer kurz behairt, an den oberen Blättern oft sehr verkïr\%. Blatthäutchen 2-4 mm lang. stumpflich. Rispe gestreckt, mit schräg aufrechten, kurzen Ästen. Äste mit bis vier grundständigen Zweigen und zwei bis drei (bis sechs) Ährehen. Ährehen zwei- bis drei- (bis vier-mblütig, bis 1,5) cm lang. Untere Hüllspelze meistens ein- selten drei-) nervig. 9-10 mm lang: obere Hü̈lspelze 11-14 mm lang, dreinervig. beide häutig. wimperharig. Deckspelzen unterwärts grimlich, schwachnervig, oberwärts häutig, mit geknieter. gedrehter, rïckenständiger, $1.5-2 \mathrm{~cm}$ langer Granne. Ährchenachse unter jeder Blüte mit kurzen, weißen Haaren. Blüte Mai bis Juli.

Anf feuchten bis trockenen Triften und Wiesen, an Wegrändern und in (iebüschen, an tbhängen und Ufern im östlichen (iebiet nicht selten. im westlichen zerstreut, auf den Nordtriesischen Inseln selten und nur auf sylt (s.chiöt\% Tidensk. Medd. 141 [1861]) und Helgoland (Hallier): an der Nordspitze (Brody!) (nach Ascherson verschleppt).

Schon Weber nennt das Gras für unser Gebiet (Prim. Fl. Hols. 9 [1780]).

\section{Formen.}

1. Blattscheiden und -spreite behaart:

f. typica Beck Fl. r. Nied.-Oesterr. I. 73 (1890), - Häufigste Form.

\section{Daz11:}

f. compacta nor. f. - Stengel $0,2-0,3 \mathrm{~m}$ hoch; Blattfläche vielmals küuzer als die Blattscheide; Rispe mit gedrängten, kurzgestielten, rorwiegend zweiblütigen Ährehen. Alsen: zwischen Sonderburg und dem Süderholz auf trockenem Sandboden 1910!!

- Blattscheiden und -flächen sämtlich kahl:

f. alpina Gaud. Fl. Helv. I. 334 (1828).

Selten. - Lübeck (Prahl 1892! als f. glabrescens Reichenb.): Schlutup (J. A. Schmidt 1867!). - Eine Ubergangsform mit 
kahlen Scheiden und schwach behaarten Spreiten sammelte Zimpel 1891! zwischen Hellbrook und Farmsen bei Hamburg.

Exemplare mit dreinerviger unterer Hüllspelze erimnern an A. amethystina Clarion, eine alpine Unterart der A. pubescens (Kiel: Mönkeberger Strand, leg. A. Christiansen!).

82. (s). 26). Avena pratensis L. Spec. plant. ed. 1. $80(1753)$.

4. Stengel aus dichtrasiger Grundachse starr aufrecht, $0,3-0,8 \mathrm{~m}$ hoch, menter der Rispe rauh. Blattscheiden lang, schwarh rauh bis fast glatt. Blattfläche 1,5-3(-4) mm breit, gefaltet, oberseits rauh, kahl. Blatthäutchen $1-4 \mathrm{~mm}$ lang, spitzlich, zerrissemrandig. Rispe 5-12 cm lang, zusammengezogen. Äste fast aufrecht. mit ein bis drei Ährchen. unter den Ährchen verdickt. Ährchen drei- bis fünf- lbis sechs-bblütig. 1,5-2 cm lang. Untere Hüllspelze 12-13 mm lang, dreinervig, obere Hüllspelze 17-19 mm lang. dreinerrig. beide häutig, spitz. Deckspelzen unterwärts grünlichweib, olswärts breit hautrandig, mit bis ïber $2 \mathrm{~cm}$ langer, geknieter, gedrehter, zusammengedrückter, oft gekrümmter, nahe der Spelzemmitte entspringender Grame. Ährchenachse kurz behart. Blüte Mai bis Juli.

An trockenen, kurzrasigen Hügeln und Abhängen, an Wegen und anf alten, bewachsenen Dünen durch das Gebiet zerstrent, streckenweise fehlend.

L a uenburg: Kil. GrönautLuther, Hb. Klatt nach Friedrich). Ha m burg: am Bahndamm zwischen IIttlerer Landweg und Billwärder Moorfleth!!. Lübeck: Schlutup (Nolte 1820 !. 1821!, J. A. Schmidt 1867!), an der Trave ron Herrenwiek abwärts (Häcker 1861!. Prahlı!!. auf dem Priwall bei Travemïnde (Nolte 1864! im Hb. Hinrichsen, Laban 1887!)!!, Waldhusen (Häcker 1828!). Oldenburg i. H.: am „Rauhen Berge“ bei siggen (Prehn!), Rossee (Prahl 1894!), Heiligenhafen (Nolte 1831 !!!!, Dazendorf. Kembs, Nen-Teschendorf, Weidehof!!, am W'ienberg (.I. A. Schmidt 1867!) mnd in der Brök (Borchmamn!!. Plön: „Lippe" bei Hohwacht!!. Angeln: Gelting-Birk (Hansen 1838!). Itzehoe: zwischen Oesan, Peissener Pohl (Brick!) und Springhoe hänfig (Prahl 1887!), zwischen Bücken (J. Schmidt!) und Hohenaspe!!. Rendsburg: Hohenhörn (J. Schmidt). Dithmarschen: Krumstedter Tierth bei Meldorf (Prahl 1887!), zwischen Süderhastedt und Eggstedt häufig (J. Schmidt!). Husum: Königsmark ((allsen), zwischen Schauenthal und Horrstedt (A. Christiansen 1908!). Tondern: Jerpstedt (A. Christiansen 1910!). Föhr: Südstrand-Wyk!!, südlich von Witsum und Hedehusum 1910!!. Amrum (Nolte 1825!): Nebel (Hb. Artsten nach r. Fischer-Benzon)!!. Norddorf!!. Sylt: zwischen Westerland und Tenningstedt (v. Fischer-Benzon 1874!), List (Schiötz). 
Die erste Notiz gibt für das besprochene Gebiet Weber (Prim. Fl. Hols. $9[1780])$.

Eine Reihe von weiteren Angaben ist falsch oder unsicher, so z. B. die der Hanburger Floren. Nach Sickmann (Ėmumeratio 9 [1836]) wurle A. pratensis zwischen Steinbek und Boberg gefunden, nach Sonder (Fl. Hamb. 49 [1851]) einzeln am hohen Elbufer und bei Trittau (hier von Lohmeyer). Besonders die beiden ersten Orte sind seither sehr häufig besucht worden; 1. pratensis fand sich an ihnen nicht wieder. Belege fehlen. Dabei ist auffällig, daß im $\mathrm{Hb}$. Lübeck ein mit A. pratensis bezeichnetes Exemplar der 4. pubescens liegt, welches Sonder bei Hamm sammelte, allerdings schon 1834!. Labans Fundort: Steinbeker Moor besitzt nach O. T'. Timm A. pubescens; des gleichen Verfassers Standort: Hinschenfelde bezieht sich nach Pflanzen im Hb. Laban! ebenfalls auf diese Art (die bei Hinschenfelde bereits J. J. Meyer 1816 sammelte!). Unrichtig ist weiter eine Standortsmitteilung "Berliner Tor" (A. Junge Ver. Naturw. Unterh. Hamb. 98 [1890]); das Exemplar ist A. elation!. Hübener sagt (Fl. v. Hamb. 497 [1S+7]): Flottbek und bemerkt dazu: „im Gebiete der Agronomie gebaut". Eine Bemerkung zu dieser Bemerkung erübrigt sich. An den Brhndamm der Berliner Bahn ist das Gras rielleicht mit Sand ron den Elbhöhen bei Ladenbeli unweit Bergedorfs verschleppt worden. Eine Reihe unkontrollierbarer Angaben hat Knuth (Fl. v. Schlesw.-Holst. 761 [1887]) für Segreberg (Thun), Eutin (ob von Lienau?), Husby (Bertram) und Flensburg (WVeidemann). Nicht mehr im Gebiete liegt das Dorf Bäk bei Ratzeburg (Klatt Fl. v. Lauenb. 170 [1865]). Auf Helgoland wurde nicht A. pratensis beobachtet (Brody nach Knuth Allg. Bot. Zeitschr. IV. 110 [1898]), sondem Trisetum flavescens (vgl. Ascherson Wiss. Meeresunters. IV. 1. $101[1900])$.

Formen.

Nicht beobachtet.

29. Gattung.

\section{Trisetum.}

Persoon Syn. I. 97 (1805).

1. Pflanze 4, lockerrasig, mit kriechender Grundachse............. pratense.

- PHanze $\odot$; Stengel einzeln oder zu wenigen ................ paniceum.

83. (qsp. 8). Trisetum pratense Pers. Syn. I. 97 (1805).

4. Stengel aus lockerrasiger frundahse autrecht oder aufsteigend. 0,3-0,7 m hoch, an und dicht unter den Knoten behaart, sonst kahl, glatt. Lntere oder alle Blattscheiden behart. obere öfter kiahl. Blattspreite flach, 1-- + mm breit, unterseits stärker, oberseits schwächer behalut. Blatthäutchen etwa $1 \mathrm{~mm}$ lang, breit. Rispe $5-12 \mathrm{~cm}$ lang, bis $2 \mathrm{~cm}$ breit, mit aufrecht wenig abstehenden, bis $2.5 \mathrm{~cm}$ lingen. rom tirunde mit Ährehen besetzten Ästen. ت̈hrehen zwei- bis drei- bis vier-iblütig. 5--6.5 mm lang. Hüllspelzen sehr ungleich. untere $\cdot 2.5-3 \mathrm{~mm}$ ling. lineal, 
eimnerrig, obere 4,5-5,5 mm lang, dreinerrig, breit lanzettlich. Deckspelze 4-5 mm lang, zweispitzig, über der Rückenmitte mit geknieter, 4-7 mm langer Gramne, undeutlich fünfnervig. Haare der Ährchenachse $1(-1,5)$ mm lang. Blüte Mai bis Juli.

An Weg- und Ackerrändern. auf Feldrainen, seltener auf Wiesen, durch das Gebiet zerstrent und oft unbeständig; mit Gras- und Kleesaat eingeführt; auf den Nordseeinselı bisher nur auf Föhr: bei Toftum 1910!! und Helgoland (Brody!).

Die erste Literaturangabe des Gebiets hat Buek (Hoppe Bot. Taschenb. 92 [1801]). Das älteste gesehene Exemplar sammelte Bargum 1803 bei Apenrade!.

\section{Formen.}

1. Blattscheiden behaart:

f. villosum Celakovsky Wiss. Ges. Sitz.-Ber. 180 (1887).

Zerstreut. - Zerfällt:

f. hutescens Aschers. Fl. Brand. I. 830 (1864). - Ährchen grünlichgelb, seltener fast gelb. - Verbreitetste Form.

f. variegatum Aschers. a. a. O. I. 830 (1864), - Ährchen violettgrünlich. - Hamburg: am Winterhuder Alsterufer (C. Timm).

- Blattscheiden kahl:

f. glabratum Aschers. Fl. Brandenb. I. 830 (1864).

Selten. - Ki e l: Neumühlen und Rotenhahn(A.Christiansen 1907!). Angeln: Ausacker (Hansen 1867!). Dithmarschen: Burg (Zimpel!), - Ein schwach behaarter Übergang Schleswig (Hinrichsen 1852!).

84. (adv. 50). Trisetum paniceum Pers. Srn. I. 97 (1805).

$\odot$. Stengel zu mehreren, in der Regel aufsteigend, 0,1-0,3 m hoch, glatt. Blattscheiden eng, langharig. Blattfliche $1-3 \mathrm{~mm}$ breit, beiderseits behaart. Blatthäutchen kurz, breit. Rispe $3-5(-8) \mathrm{cm}$ lang, $1-1,5 \mathrm{~cm}$ breit, dicht, mit $0,4-1,5 \mathrm{~cm}$ langen Ästen. Ährehen $3-5(-6) \mathrm{mm}$ lang, mit (zwei bis) drei bis fünf Blüten. Untere Hüllspelze $2--2,5 \mathrm{~cm}$ lang, lineal, eimnervig, obere $3 \mathrm{~mm}$ lang, lanzettlich, dreinervig. Deckspelzen bis $3 \mathrm{~mm}$ lang, auf dem Rücken ratuh. mit undentlichen Nerven, nit im oberen Viertel des Spelzenrückens entspringender, bis 3 mm langer Granne. kurz zweispitzig: Ährchenachse behaart. Blïte Juli.

Auf Schutt bei Hamburg selten.

Hamburg: bei der Wollkämmerei am Reiherstieg (J. Schmidt 1897!). 
30. Gattung.

\section{Ventenata.}

hoeler Descript. Gram. 273 (1802).

85. (adr. 51). Ventenata dubia F. sichultz Pollichia XX, XXI. 273)(18(i3).

$\odot$. Stengel zu mehreren, aufrecht oder aufsteigend, $0.2-0.8 \mathrm{~m}$ hoch. glatt. Blattscheiden in der Regel glatt, seltener rauh. Blattfläche 1--2(-3) mm breit. oherseits rauh. oft getaltet. Blatthäutchen $2-9 \mathrm{~mm}$ lang, spitzlich. Rispe 5-111-201) cm lang. seln locker. dlüm- und linngästig. Ährchen zwej- bis dreiblütig. 9--11 mm lang. Hüllspelzen spitz, untere 5-6 mm ling. siebennervig. am Kiel rauh, obere s mm lang. neunnerrig, an den Nerven rauh. beide hautrandig. Lntere Deckspelze bis $11 \mathrm{~mm}$ lang. undeutlich nervig, gramenartig spitz, obere bis 9 mm lang. gramenartig-zweispitzig. aus der Rückemmitte mit geknieter. bis $13 \mathrm{~mm}$ langer Granne. Ährchenachse kahl. Blüte Mai bis Juli.

Auf Schutt bei Hamburg selten.

Hamburg: bei der Wandsbeker Dampfmühle (.T. sichmidt. Mai 1s!4!; Zimpel, Juni 1894!).

Die sonstigen Florenangaben dieser Art für Hamburg oder Holstein sind unsicher oder unrichtig. Weber hat in seiner Aufzählung Holcus biaristatus (Prim. Fl. Hols. 72 [1780]), den schon Hornemann (0ec. Plantel. 3. Udg. I. 131 [1821]) zu Avena tenuis Inch. = Tentenata dubia F. Schultz gestellt hat. Bezeichnung des Fundortes und Belege fehlen. Yach ihm will sickmam das Gras bei Friedrichsul gefunden haben (Enumeratioy [1836]); den späteren Hamburger Floristen. selbst dem unsicheren Hübener. fehlt es (rgl. auch Koch Srn. Deutsche u. Schweiz. Fl. 797 [1838]). Moblers Hinweis (Handb. Gewächsk. 147 [1815]) "Holstein" geht wohl auf Webers Notiz zurück. Noltes Herbar enthält die Art: "Um Kiel" gesammelt!, wahrscheinlich (nach Prahl Krit. Fl. 25t [1890] sicher) im Botanischen Garten. Im Hb. J. A. Schmidt liegt ein Exemplar von Hansen, der die Pflanze auscab) (Herh. schlesw.-Holst.-Lauenh. Fl. 1155 [1853]). ihm .ohne spezielle Angrabe des Fundorts" ron Nolte übergeben!. (Eine Pflanze des Bogens ist Trisetum pratense!.) Unglaubwürdig ist eine netere Angabe, nach der Engelken $V$. dubia zwischen Gettorf und Wulfshagener Hütten im dïnischen Wohld gefunden haben will. Lie nürdliche Verbreitungsgrenze der Art verläuft südlich rom Harz.

31. Gattung:

\section{Aera.}

L. Gen. plant. ed. 5. 31 (175t) z. T.

1. Pflanze $\odot$, zart, mehrstengelig; Deckspelzen bis $1,5 \mathrm{~mm}$ lang.......... 2

- Pflanze 4 , dichtrasig; Deckspelzen $2-4 \mathrm{~mm}$ lang ................ 4 
2. Ährchen bis $1,5 \mathrm{~mm}$ lang, an feinen Stielen in sehr lockerer Rispe.

- Ährchen 2-2,5 $\mathrm{mm}$ lang

A. capillaris.

3. Rispe locker, mit abstehenden Ästen; Ährchen voneinander entfernt.

A. caryophyllea.

- Rispe dicht, mit aufrechten, kurzen Ästen; Ährchen sehr genähert..A. praecox.

4. Deckspelze mit deutlich geknieter Granne......................

- Deckspelze mit gerader oder undeutlich geknieter Granne ............. 6

5. Blatthäutchen 2-3 mm lang, stumpf bis spitzlich; Blätter derb...A. Alexuosa.

- Blatthäutchen 3-8 mm lang, spitz; Blätter der nicht blühenden Triebe haarfein. . A. setacea.

6. Ährchen 2-4 mm lang; Granne die Deckspelze nicht überragend.

A. caespitosa.

- Ährchen 4-6 mı lang; Granne die Deckspelze überragend ....A. Wibeliana.

86. (adv. 52). Aera capillaris Host Gram. Anstr. IV. 20 (1809).

$\odot$. Stengel zu mehreren, meistens aufrecht, $0,1-0,3 \mathrm{~m}$ hoch, glatt, fein. Blattscheiden etwas rauh. zuweilen glatt. Blattflache schmal, gefaltet, schwach rauh. kilıl. Blatthäutchen 1-3 mm lang, spitzlich. Rispe bis fast $10 \mathrm{~cm}$ lang, lockel ausgebreitet, mit haarfeinen, geschlängelten. langen Isten. Ährehen lang gestielt. $11-11.5 \mathrm{~mm}$ lang. Hüllspelzen fast gleich lang, ein- oder drei-nervig. häutig. Dechspelzen etwa 1 mm lang, die der unteren Blïte spitz, gramnenlos, die der oberen Blüte mit bis über $2 \mathrm{~mm}$ langer, geknieter (Aranne. Ährchenachse behart. Blüte Juni.

Auf Schutt bei Hamburg selten.

Ha mburg: Winterhnder Bruch (.J. schmidt 18! 10 !; Ber. Deutsche Bot. Ges. X. [86] [1892]).

\section{7. (sp). 27). Aera caryophyllea L. Spec. plant. ed. 1. 66 (1753).}

$\odot$. Stengel in der Regel zu mehreren (bis vielen), aufrecht, glatt, 0,05-0.3 m hoch. Blattscheiden glatt oder sehr schwach rauh. Blattfläche $1(-2) \mathrm{mm}$ breit, oft gefaltet, oberwärts rauh. Blatthäutchen 3- $4(-5) \mathrm{mm}$ lang, spitz. Rispe 2,5-6 cm lang, mit aufrecht abstehenden. verlängerten, rauhen Ästen. Ährehen $2,5 \mathrm{~mm}$ lang. Hüllspelzen fast gleich, spitz, einnervig, am Kiel rauh. Deckspelzen etwa 1,5 mm lang: fein-ranh, zweispitzig, im unteren Drittel mit geknieter, bis $3 \mathrm{~mm}$ langer Gramme. Ährehenachse behart. Blüte (April) Mai bis Juni, selten später.

Auf sandigem Boden auf Äckern, Feldern, Weiden, an Wegen und Abhängen durch das Gebiet nicht selten oder häufig; auch auf 'Föhr, Amrum, Sylt und Röm.

Als gemein in Holstein nennt Oeder (Flora Danica t. 382 [1768]) unsere Art. 


\section{Formen.}

f. gemina A. u. Gr. Syn. Mitt.-Europ. Fl. II. 1. 282 (1899)。 - Bei uns sicher nur in dieser Form.

Dazu:

f. typica A. u. Gr. a. a. O. II. 1. 282 (1899). - Ährehen in der Regel zweiblütig. Blüte Mai und Juni. - Häufigste Form.

f. Degenkolbii A. 11. Gr. a. a. O. II. 1. 283 (1899). - Ährehen in der Mehrzahl einblïtig. Blüte August und September. Kiel: Labö (Degenkolb).

88. (sp. 28). Aera praecox L. Spec. plant. ed. 1. 65 (1753).

$\odot$ und $\odot$. Stengel zu mehreren, meistens aufrecht, $2-25 \mathrm{~cm}$ hoch, glatt. Blattscheiden oberwäts dentlich rauh, die oberen schwach aufgeblasen. Blattfläche etwa $1(-2) \mathrm{mm}$ breit, gefaltet. Blatthäutchen 1-2 $\mathrm{mm}$ lang, spitzlich. Rispe 1-2(-5) cm lang, mit aufrechten, kurzen. wenigährigen Ästen, dicht, selten etwas locker. Ährchen 2,5 mm lang. Hüllspelzen fast gleich, länglich. am Rürcken grün, sonst häntig. Deckspelzen häntig, spitz, aus dem unteren Drittel mit 3-4 mm langer, schwach geknieter Gramme. Ährchenachse nur unter den Blüten behaart. Blüte April bis Juni.

Auf sandigem Boden der Äcker, Triften und Heiden, an Wegen und in Gräben häufig; auch auf den Nordfriesischen Inseln verbreitet.

Mit voriger Art schon von Oeder (Flora Danica t. 383 [1768]) als in Holstein und Schleswig rorkommend erwähnt.

\section{Formen.}

Tom Typus weicht ab:

f. decumbens nov. f. - Stengel niederliegend, nur 2-3 cm lang; Rispe bis $1 \mathrm{~cm}$ lang. - Lauenburg: anf Waldwegen im Stubbenteich bei Roseburg!!.

89. (sp. 29). Aera flexuosa L. Spec. plant. ed. 1. 65 (1753).

4. Grundachse dicht- bis lockerrasig. Stengel aufrecht oder aufsteigend, 0,3-0,6 m hoch. glatt. Blattscheiden glatt oder schwach rïckwärts rauh. Blattläche schmal, eingerollt, oberwärts rauh. Blatthäutchen 2-3 mm lang, stumpf. Rispe $6-15 \mathrm{~cm}$ lang, mit verlängerten, geschlängelten Ästen mit ein (bis zwei) grundständigen Zweigen. Ährehen filst $5 \mathrm{~mm}$ lang. Hüllspelzen 4.5 und $5 \mathrm{~mm}$ lang. lanzettlich, oberwärts oder völlig häutig. spit\%, einnervig. Deckspelzen etwa $13.5-14$ mm lang. gestutzt, vom Rüekengrunde mit $5-6 \mathrm{~mm}$ langer, geknieter Grame. 
Thrchenachse behaart, zwischen den beiden Blüten wenigstens fünfmal so kurz wie die obere Deckspelze. Blïte Juni bis Juli.

Auf sandigem und moorigem Boden ron Heiden, Triften, Wäldern und Mooren. weniger in Sümpfen, häufig. auch auf den Nordfriesischen Inseln Föhr, Amrum, Sylt und Röm.

Die erste Erwähnung der Pflanze bringt Weber (Prim. Fl. Hols. 9 [1780] als Acenu montenu $\beta$. adultior. Bei Esmarch (Progr. Domschule Schlesw. 14 [1789]) wird A. montrner fälschlich als jüngere Pflanze oder Spielart von A. Alexuos bezeichnet.

\section{Formen.}

1. Grundachse dicht- bis wenig lockerrasig ............ 2

- Grundachse sehr lockerrasig:

f. Buchenavii A. u. Gr. Syn. Mitt.-Europ. Fl. II. 1. 287 (1899). Stengel am Grunde knickig niedergebogen, aufsteigend.

In Sümpfen selten. - Stormarn: Ahrensfelder Sumpfwiesen!!. Eutin: Bujendorfer Moor!!. Kiel: Grevenkrug!!, am Drecksee und Meimersdorfer Moor (A. Christiansen!). Röm: mehrfach in Mooren!!.

2. Rispe groß, locker, mit deutlich geschlängelten Ästen:

f. typica Beck Fl. Nied.-Oesterr. I. 68 (1890). - Gewöhnliche Form.

Dazu:

f. Legei Richter Plant. Europ. I. 57 (1890), - Hüllspelzen weißlich - durchscheinend; Ährchen und Rispenäste weißlich. - An schattigen (feuchten) Orten zerstrent.

- Rispe znsammengezogen, dicht. mit wenig geschlängelten Ästen:

f'. montana Parl. Fl. Ital. I. 241 (1848). - Boreal-alpine Form. Selten. - Hadersleben: auf feuchtem Heideboden bei Brönsmühle (Borst 1887!).

90. (s). 301. Aera setacea Huds. Fl. Angl. ed. 1. 30 (1762).

7. Grundachse dichtrasig. Stengel aufrecht oder aufsteigend, $0,2-0,7 \mathrm{~m}$ hoch, glatt. Blattscheiden eng, glatt. Blattfläche $1-2 \mathrm{~mm}$ breit, in der Regel gefaltet, an den nichtblühenden Trieben zahlreich, haarfein. Blatthäutchen $3-8 \mathrm{~mm}$ lang. spitz. Rispe $5-15 \mathrm{~cm}$ lang, mit rerlängerten, aufrecht fast anliegenden. geschlängelten Ästen. Ährchen 13- $4 \mathrm{~mm}$ lang, grünviolett, oben breit. Hüllspelzen fast gleich lang, untere schmäier, eimnerrig. obere breiter, dreinervig. Deckspelzen 2-2,5 mm lang, rauh, mit am Rücken fast grundständiger, geknieter Grame von bis $4,5 \mathrm{~mm}$ Länge, die obere doppelt so lang wie das Achsen- 
glied unter ihr. Ïhrehenachse mit 1 mm langen Hatren, ïber die obere Blïte verlängert mol hier selten mit ziemlich cutwickelter dritter Blite (Röm [Borst 1869!, Hb. Kopenhagen]). Blüte Juli und August.

An sumpfigen Orten der Heide- und Dïnenmoore (gem an Gräben, Thimpeln und soens in westlichen und mittleren schleswig zerstrent, in Holstein sehr selten.

Holstein: Stormarn: am Helkenteich bei 'Trittau (Prahl 1887!)!!.

Schleswig: Eidorstedt: St. Peter Nolte, Hi). Kopenhagen!, F. r. Mü̈ller 18t5!), sïderhöft (Hansen, Hb. Hamb.!). Husum: Hockensbüll, Horrstedt, ()lderup! und Hohlacker (v. Fischer-Benzons. Flensburg: zwischen Bülderup und Bau (Prahl 1893!. 1894!). Tondern: am Langenberge bei Leck (Prahl!), Schardebüllfeld 1. Christiansen!). Gallehums (Prahl!), schadser IIoor 1896! und Hedegaded 1906! (.J. Sichmidt), an den Soller Seen bei Jerpstedt (Prahl!). Föhr: siidlich und westlich der Laurentiuskirche mehrfach 1910!!. Amrum: bei der Togelkoje t.J. schmialt 1906!)!!, in Dünentälern sïdlich von Norddort!!. Sylt: Wassertal auf Hörnum!!, am sïdrand der Morsmmheide!!. Rïm (Hansen 1846)!, 1853!) mehrfach!!.

Die Art, von Hornemam zuerst für das Gebiet aufgefülırt, ist für Holstein mehrfach genannt worden. Im Kopenhagener Herbar liegt ein Exemplar, bezeichnet „... i Holsteen", "leg. Nolte". Die Aufschrift stammt nicht von Nolte; eine Verwechslung mit schleswigschen Exemplaren ist deshalb wohl möglich. Hübener hat (Fl. v. Hamb. 496 [1847]) Schöningstedt, das Stellinger Moor, Schenefeld und die Harksheide als Orte des Auftretens; an letzterer Stelle soll die Art häufig sein, doch gibt es dort jetzt nur A. Alexuosa. Schon Sonder bezweifelt die Richtigkeit von Hübeners Angaben, von denen keine durch Funde anderer Floristen bestätigt werden konnte. Lienau sagt (Fl. Fürstent. Lüb. 6 [1863]): „Soll auf dem Süseler Moore vorkommen." Daraus macht dann Laban (F1. v. Holst. 229 [1866]) bestimmt: Süseler Moor. Eine Angabe für Segeberg: Strenglin ist vom Beobachter zurüchgenommen worden.

\section{Formen.}

f. Immill J. Sichmidt Allg. Bot. Zeitschr. XIII. 7 (1907). - - Stengel niedrig, bis zur Rispe beblättert, nur doppelt so lang wie die Grundblätter, $0,2-0,3 \mathrm{~m}$ hoch; Ährchen nur $3 \mathrm{~mm}$ lang.

Tondern: Hedegaard (J. Schmidt 1906!). Amrum: Norddorf!!. Sylt: Wassertal auf Hörnum!!. Röm (Hansen 1846!): Westerhede (Prahl 1905!).

Im Lübecker Herbar liegt die Form aus Ostfriesland: Wittmund (LantziusBeninga!).

91. (s]).31). Aera caespitosa L. Niec. plant. ed. 1. $64(1653)$.

4. Grundachse dichtrasig. Stengel meistens aufrecht, $(0,2-) 0,4-1$ $(-1,5) \mathrm{m}$ hoch, meistens röllig glatt. Blattscheiden eng. in der Regel 
glatt. Blattspreite meistens flach, $(1-2)-4 \mathrm{~mm}$ breit, oberseits mit sieben stark rauhen Nerven, unterseits glatt. Blatthäutchen 2-5(-8) mm lang, spitz. Rispe bis $20 \mathrm{~cm}$ lang, mit rerlängerten, ausgebreiteten, kaum geschlängelten Ästen. Ährchen 11,5-13-4 mm lang. Untere Hüllspelze kürzer, schmäler, spitz, einnervig. obere breiter, dreinervig. spitzlich. Deckspelzen gestutzt, gezähnelt, etwa $2 \mathrm{~mm}$ lang, mit geknieter, grundständiger Granne. Granne so lang oder kürzer als die Deckspelze. Ährehenachse mit bis 1 mm langen Haaren. Blüte Juni und Juli, zuweilen später.

Auf trockenen bis feuchten Wiesen, an Abhängen, in Gebüschen und in Wäldern durch das Gebiet häufig, doch auf den Nordfriesischen Inseln nur auf Helgoland (Brody!. Ascherson), Föhr: Oevenum und Alkersum!!, Nieblum (Kertelheim nach Kinuth, als. f. gigmuteri), sylt (schiötz): Keitum (Ostermeyer)!! und zwischen Westerland und Timum!! und Röm: zwischen Havneby und Kirkeby (Raunkiaer).

Schon Weber zählt (Prim. Fl. Hols. 7 [1780]) A. caespitosa auf.

Während Schiötz (Naturh. Foren. Videnskab. Medd. 141 [1861]) für Sylt A. caespitosa nemnt, fehlt bei ihm die ungleich häufigere A. Alexuosa, so daß seine Notiz möglicherweise unrichtig ist. Brody hatte seine Helgoländer Pflanze als $f$. brevifolia bestimmt; diese Bestimmung veröffentlichte Knuth (Allg. Bot. Zeitschr. IV. 109 [1898]). Nach Ascherson und Graebner (Syn. Mitt.-Europ. FI. II. 1. 292 [1899]) ist aber die Helgoländer Form nicht mit der genamnten borealen Rasse identisch.

\section{Formen.}

1. Ährchen 3,5-4 mm lang, meistens zweiblïtig ............ 2

- Ährchen 1,5-2,5 mm lang, oft einblütig:

f. parviftora Richter Plant. Europ. I. 56 (1890).

Ziemlich selten. - Lauenburg: mehrfach im Sachsenwalde!!. Ha mburg: Elbufer und Elbinseln (Sonder Fl. Hamb. 43 [1851]) z. B. M[oorwärder!!. Pinneberg: Teufelsbrück (C. Timm; bei f. altissima). Husum: Immenstedter Holz (A. Christiansen!).

Von den als A. parriftora bezeichneten Nolteschen Pflanzen gehört nur die eines Standorts wirklich hierher!; dieser Ortsname ist aber ganz unleserlich.

2. Blätter flach:

f. gemina Rehb. Icon. Fl. Germ. et Helv. I. t. XCVI. fig. 1682 (1834), - Häufig.

Dazu:

f. altissima Aschers. Fl. Brandenb. I. 833 (1864). - Rispe groß, ausgebreitet, mit verlängerten, schlaffen Ästen; 
Deckspelzen oberwärts gelblich oder grünlich. - In fenchten Wäldern und Gebüschen nicht selten.

f. aurea Wimm. u. Grab. Fl. Siles. I. 60 (1827). - Rispe wie beim Typus, etwas ausgebreitet, mit kürzeren, strafferen Ästen; Ährchen goldgelb gefärbt.

Annäherungsformen (z. T. ron $f$. altissima): Lauenburg: an der Horster Chaussee!! Kiel: Exerzierplatz, Meimersdorfer Moor, Gettorf (A. Christiansen!). Flensburg: Jerrishoe (A. Christiansen!).

Unsere Formen entsprechen anscheinend (nach Diagnose) einer Abart, welche von Kneucker (Allg. Bot. Zeitschr.XII. 285 [1906]) bezeichnet wird: „forma inter $f$. altissimam (Moench) et $v$. auream Wimm. et Grab.". In der Ährchenfarbe erimern sie etwas an f. ochrolenca (Rehb. Icon. fig. 1685 [1834]).

- Blätter gefaltet oder eingerollt:

f. setifotia Bischoff in Koch Syn. Fl. Germ. et Helv. ed. 2. 914 (1844).

Auf trockenem Boden selten. - Stormarn: Wandsbek (J. A. Schmidt 1868!). Kiel: Schönberg (J. J. Meyer 1830!).

\section{Mißbildungen}

f. m. vivipara Rchb. a. a. O. fig. 1684 (1834). - Ährchen in Laubsprosse auswachsend.

Hamburg: Wollkämmerei am Reiherstieg (Laban 1895!), Moolwärder (J. Schmidt)!!. - Abgebildet schon durch Oeder (Flora Danica t. $240[1765])$.

92. (s1). 32). Aera Wibeliana sonder in Koch sin. Fl. Ferm. et Helr. ed. 2. 915 (1844).

4. Grundachse mit kurzen oder bis $6 \mathrm{~cm}$ verlängerten Gliedern. stengel aufrecht, ziemlich derb, $(0,2-10,4-0,9 \mathrm{~m}$ hoch, glatt. Blattscheiden glatt. Blattfliche $(1-2-4 \mathrm{~mm}$ breit, flach oder gerollt. Blatthäutchen 3-5 mm lang. spitz. Rispe $8-20$ cm ling, etwas schlaffastig. Ähchen (4-5-6 mm lang. Hüllspelzen etwas ungleich, spitz. lanzettlich, untere kürzer, einnervig, obere dreinervig. Deckispelze der unteren Blïte $t$ mm, der oberen $3 \mathrm{~mm}$ lang, fünfnervig, beide stumpf, an Ende gezähnelt, mit rülekenstïndiger, die spelze bis 1 mm ïberragender Granne. Ährchenachse beharrt, über die obere Blüte stark verlängert. Blüte Juni, selten September.

Auf Sand- und 'Tonboden, seltener an Deichen, an vom Wechsel des Ebbe- und Flutwasiers getroffenen Orten am Lnterlante der Elbe 


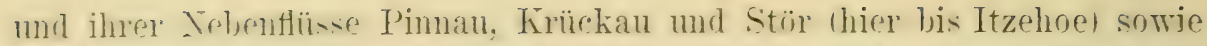
am Lutrilatufe der Eidre rom Rendshurg abwäts nicht selten. stellenweise

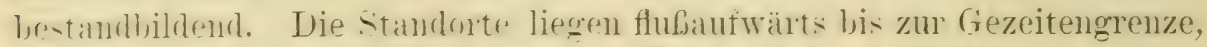
flußabwärts bis zur Einflußgrenze des Meerwassers.

Zu dieser Art zählt A. paludosa Wibel (Prim. Fl. Werth. 113 [1797]), ron Altona crenanut. Er sagt ron der Pflanze, sie zeige "folia latiora". Auch Koch weist (a. a. 0. $915[1814]$ ) auf die Blätter hin mit der Bemerkung: „foliis planis". Tatsächlich sind die Blätter durchaus nicht immer flach, eine Erscheinung, auf die schon G. F. W. Meyer hinweist (Chlor. hannov. 639 [1836]); er reiht die Pflanze als: "Spielart a. Huvictilis" unter A. caespitosa und bemerkt: „Die Spielart erreicht nicht selten eine Höhe von 3 Fuß und ist durch gröbere, gleichmäBig bleichgelbe Blütchen ausgezeichnet. Nahe am Wasser wachsend, sind ihre Blätter breit und völlig flach, entfernter ron diesem werden sie

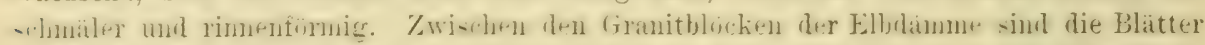

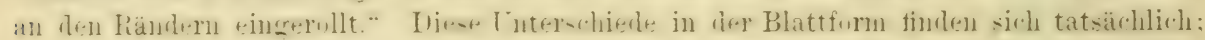
in der Pegel sind alle Blätter flach; an trockenen Orten sind die Stengelblätter gefaltet

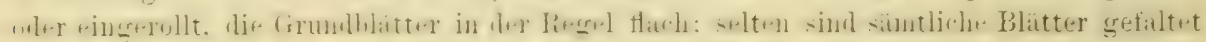
oder eingerollt. Ifever bemerkt weiter: ,Auch die Witterung und Tageszeit ist hierauf ron Einfluœ; im heiben Mittage sind die Blätter auf trockenem Standorte völlig eincewickelt". Diese Beobachtung rermag ich nicht zu bestätigen. Ebensowenig konnte ich trotz Beobachtung an zahlreichen Stellen etwas ron den angeblich nicht seltenen Überträngen nach A. caespitosa bemerken. Meyer sagt darüber: "Mit zunehmender Trockenheit des Bodens tritt allmällich die gewöhnliche bunte Färbung der Blättchen ein und

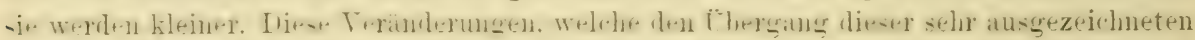
Form in die grewöhnliche A. caespitosa nachweisen, kann man in günstiger Lokalität rom Wasserstande ab in 10-20 Schritt Entfernung verfolgen". Tatsächlich spielen die Spelzen an solchen Standorten ins Violette, auch sind die Ährchen kleiner (4 mm); im

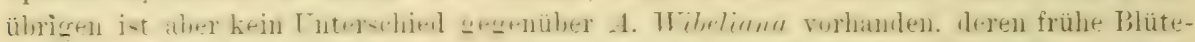

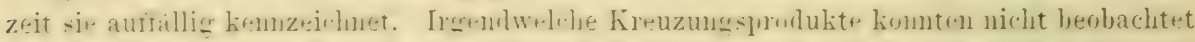
werden, dürften auch wohl wegen der späteren Blütezeit der A. caespitosa fehlen.

Ein zweites als wesentlich genanntes Merhmal ist ebenfalls unwichtig, da sich Variationen finden; es ist das das Merkmal der kriechenden Grundachse. Darauf weist bereits Döll hin (Fl. v. Baden 199 [185̃]): „Die äuberen Halme der vorliegenden Art" (A. caespitosa) , sind bei starken Rasen am Grunde etwas grebogen und schlagen daher an feuchten Orten wohl auch an den unteren Knoten Wurzeln; aber einen wirklich kriechenden Wurzelstock habe ich noch nie an dieser Art beobachtet. Dies gilt auch von A. Wibeliana Sonder, welche ich in der Gegend ron Hamburg in Gegenwart des

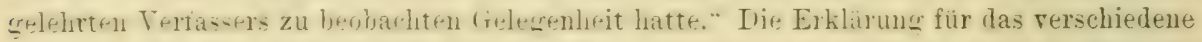
Terhalten der Grundachse (bald kurze, bald stark verlängerte Glieder) liegt in den verschiedenen Bedingungen der Standorte. Wo der Boden, wie z. B. am hohen Elbufer von Altona bis Schulau (und dort sammeln die Floristen in der Regel A. Wibeliana), durch Absturz rom hohen Cfer im Vorlande jährlich etwas erhöht wird, da folgt die Pflanze

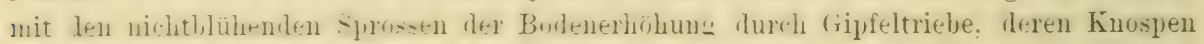

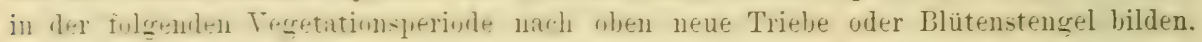
die am Grunde Wurzeln treiben. Diese Achsentriebe erinnern etwas an Ausläufer, sind alw:r lureh die War:h-tun-richtuner und dif rund um das Achsenglierl erfolgende Wurzelbildung ron ihnen sofort zu unterscheiden. Wo die Bodenerhöhung fehlt, tritt diese Achsenverlängerung nicht ein; ihr Unterbleiben ist die Regel.

Teuman will (Botaniska Not. 153 |1895]) in A. Wibeliana die Kreuzung ron A. bottnica und A. caespitosa sehen. Die Deutung ist bei dem getrennten Verbreitungs- 
gebiet der A. Wibetiana und A. bottnica wohl ausgeschlossen. Beide stehen in sehr nahen Beziehungen; ein wesentlicher Unterschied liegt aber z. B. in der längeren Granne der $A$. bottnica, welche die Hüllspelzen meistens um $2 \mathrm{~mm}$, zuweilen um $3 \mathrm{~mm}$ überragt, während sie bei A. Wibeliana die Hülspelzen nicht oder sehr wenig überragt.

\section{Formen.}

1. Achsenglieder sehr kurz, daher Pflanze dichtrasig:

var. gemina nov. var. - Ährchen (4-) $5-6 \mathrm{~mm}$ lang; Blätter sämtlich oder öfter téilweise gefaltet oder eingerollt.

Häufigere Form. Dazu:

f. setifolia nov. f. - Pflanze 0,2-0,3 m hoch; Ährchen $4 \mathrm{~mm}$ lang; Blätter sämtlich eingerollt, ziemlich derb. Glückstadt: am Steindeich bei Neuendeich!!.

- Achsenglieder gestreckt, 2-6 cm lang, daher Pflanze lockerrasig: var. Lenziana ${ }^{1}$ ) nov. var. - Ährehen $5-6 \mathrm{~mm}$ lang; Blätter flach.

Auf Sandboden am Elbufer unterhalb Hamburgs mehrfach; an der Eider bisher nur bei Rendsburg: Klint (Hansen 1853, Hb. Hinrichsen!). (Geeignete Standorte sind an der Eider selten.)

32. Gattung.

\section{Weingaertneria.}

Bernhardi Syst. Verz. Pfl. Erfurt. I. 23, 51 (1800).

93. (s). 33). Weingaertneria canescens Bernh. Pfl. Ert. כ̌1 (1800).

4. Grundachse meistens dichtrasig. Stengel zu mehreren, aufrecht oder aufsteigend. $0,1-0.3 \mathrm{~m}$ hoch, glatt. Blattscheiden meistens schwach rauh, die oberen etwas aufgeblasen. Blattliache kurz, etwas starr, gefaltet oder eingerollt, rauh. Blatthäutchen (1-)2-3 mm lang, stumpflich. Rispe $3-6(-10) \mathrm{cm}$ lang, kurzästig. Äste in der Blïte ausgebreitet, sonst zusammengezogen. Ährchen 2,5-3 mm lang. Hüllspelzen spitz, etwas ungleich, untere eimnervig. obere meistens dreinervig. Dechspelzen etwa $1.5 \mathrm{~mm}$ lang, stumptlich. ans dem Grunde des Rïlekens begramnt. Granne zweiteilig, oberer Teil weib, keulig. Ährchenachse zwischen beiden Blüten kurz feinbehaart. Blüte Juni bis Juli, selten später.

Auf sandboden der Triften. Heiden. Dünen. der Abhänge und Välder besonders im mittleren Gebiet, weniger im östlichen Hügellande zz. B. auf Alsen seltent; nicht selten bis häufig ant den Dünen der Vordfriesischen Inseln.

Zuerst genamnt von Weber (Prim. Fl. Hols. 9 [1780]) als Avenn canescens.

1) Nach Herrn Prof. Dr. Lenz † in Lübeck. 


\section{Formen.}

1. Untere Stengelglieder gestreckt: wenige am Grunde knickig aufsteigrnde. verzweigte. zuweilen wuzehte stengel an jeder Pflanze: f. maritima Godron in Godr. u. Gren. Fl. France III. 502 (1856). Blätter dicklich, auffällig starr; Blatthäutchen spitz; Rispe schmal, locker.

Auf den Dünen der Nordseeküste in Eiderstedt (schon Nolte!), auf Föhr, Amrum, Sylt und Röm zerstreut bis nicht selten; seltener auf den Ostseedünen (in Annäherungsformen auch auf Binnendünen).

An der Nordsee ist die Form oft ron selur charakteristischer Tracht; an den Übergängen der Dünen in die Heiden und Grastriften aber geht sie stellenweise allmählich in den Typus über; derartige Ülsergangsformen treten im Bimnenlande und an der Ostsee zerstreut auf, doch fehlt an der Ostsee f. maritima auch in deutlicher Ausbildung nicht (z. B. Kiel: Bottsand [A. Christiansen!]).

- Untere stengelglieder kurz. Stengel diher genähert, antrecht oder aufsteigend:

f. typica A. u. Gr. Syn. Mitt.-Europ. Fl. II. 1. 300 (1899). Pflanze grangrün.

Dazı :

f. flavescens Klinggräff 2. Nachtr. Fl. Preuß. 163 (1866). Pflanze gelbgrün. - Zerstrent, z. B. La uenburg: Grambek!!. Stormarn: Schiffbek und Wandsbek(C.Timm), Poppenbüttel (Erichsen!). H a mburg mehrfach (C.Timm). Lïbeck: Wesloe (J. Schmidt)!!. Rendsburg: Hohenwestedt (Hennings!). Kiel: Bordesholm und Einfeld! (Hennings). Dithmarschen: Westdorf bei Gudendorf (J.Schmidt!). Husum: Jägerkrug! und Flensburg: Tarp! (A. Christiansen).

f. serotina nov. f. - Hüllspelzen etwa doppelt so breit hautrandig als beim Typus. Blütezeit September. - Hamburg: Bergedorf (J. A. Schmidt 1863!).

33. Gattung.

\section{Sieglingia.}

Bernhardi Syst. Verz. Pfl. Erfurt. I. 20, 44 (1800).

94. (11. 34). Sieglingia decumbens Bernh. Pf. Erf. I. $44(1<00 \%$.

4. Grundachse dichtrasig. Stengel zahlreich, niederliegend bis auf- 
steigend, 0,1-0,3(-0,6) m hoch, glatt. Blattscheiden glatt, am Rande wimperharig, sonst kahl. Blattfläche $1-3(-4) \mathrm{mm}$ breit, am Rande

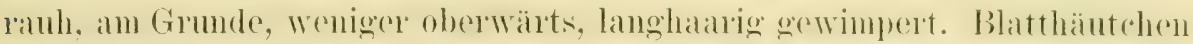
wanz undeutlich, dureh eine Hatrueihe ersetzt. Rispe kum, sehmal zusammen-

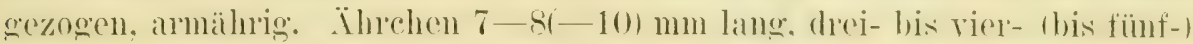

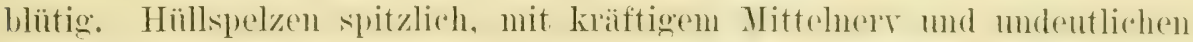
reitemueren. die untere etwa 5 , die obere etwa 7 mm lang. Dechepelzan breit, glänzend, sehr schwachnervig, dreizähnig, 4-5 mm lang, Vorspelze und Frucht umsehliebend. Alnehenarhse leicht zorfallend, unter den Blüten mit Büscheln kuzer Haare. Biüte Mai bis Juli.

Auf feuchtem bis trockenem Boden auf Mooren, Wiesen und Heiden, in Grebüschen und IV̈̈lern häufig; nicht selten lis zerstrent auf Föhr. Amrum, Sylt und Röm.

Von Weber (Prim. Fl. Hols. S [1780]) als Poc decumbens genannt.

Formen.

Nicht beobachtet.

34. Gattung.

Gaudinia.

Pal. Beauv. Agrost. 95 (1812) z. T.

95. (adr. 53). Gaudinia fragilis Pal. Beaur. Agrost. 164 (1812).

$\odot$. Stengel zu mehreren, aufrecht oder aufsteigend, bis $0,5 \mathrm{~m}$ hoch, glatt. Blattscheiden glatt, die oberste zuweilen schwach erweitert, oberste kahl, untere dicht behant. Blattfäche gevimpert oder kahl, unterseits glatt, oberseits rauh, $2-4 \mathrm{~mm}$ breit. Ährchen in einfacher Ähre. Ähre meistens 10-15 cm lang. Ährchen sehr schmal, bis $15 \mathrm{~mm}$ lang, drei- bis siebenblütig. Hüllspelzen sehr ungleich, untere schmallanzettlich, 3-4 mm lang, dreinervig. obere $7-9 \mathrm{~mm}$ lang. breiter. hantrandig. sieben- (bis neun-) nervig, an Rücken behart oder rauh. Deckspelze bis $7,5 \mathrm{~mm}$ lang, glänzend. zweispitzig, mit geknicter. oberwärts geschlängelter, rï̈lienständiger, bis $13 \mathrm{~mm}$ langer (tramme. Blïte . Juni bis Juli.

Auf angesäetem Grasland bei Hamburg. selten.

Hamburg: Eppendorf (Sonder 1831, Sickmann!).

Sickmann hat die erste Angabe des Grases (Enumeratio 9 [1836] als Avena fiagilis).

Der Standort wurle von Koch übernommen (Synops. Fl. Germ. et Helv. ed. 2. II. 950 [1846]), was schon kurz darauf Hübener (Fl. F. Hamb. VIII. [1847]) bissig monierte. 


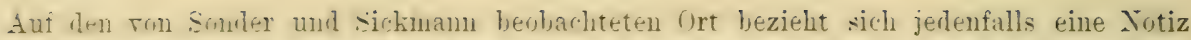
in Kingenhagener Herbar: .Terl Altona" und ,. legr. Sickmann", da dieser die Pflanze nur Fon Eppendorf nennt, wo sie jetzt schon seit Jahrzehnten rerschwunden ist (schon 1880 fehlte sie). Ein Gras im Herbar Laban, mit Gaudinia fragilis bezeichnet, ist Avena juotensis: als Fumbrt wird Eplendorf aufoeführt. wo A. pratensis nicht rorkommt. Es mub wohl irgend eine Terweclsslung vorliegen.

Nicht belegt ist eine Notiz bei Reichenbach (Vorlesungsverz. Hamb. 20 [1881]) eines Auftretens liei Tellingroütel im Alstertal. Sie greht auf einen Naclitrag Noltes in seinem Hawdexemplar dw. Noritien zurüch (Nor. Fl. Hols. zu 1.3 [16.6]): Flürge soll danach G. fragilis 1815 bei Wellingsbüttel gefunden haben.

\section{Gattung.}

\section{Arundo.}

L. Gen. plant. ed. 5. 35 (1754) z. 'T.

96. (3). 35. Arundo phragmites L. sipec. pllant. ed. 1. 81 (175:3).

7. Frmdachse bis $5 \mathrm{~cm}$ dick. lang kriechend. verzweigt. Stengel $10.15-11-3 \mathrm{~m}$ hrsch. aufreclit. selten nifederliegend idamn oft ansläuferartig sehr gestreckto. glatt. Blattscheiden derl, schwach rauh oder glatt. Blattlache lis über 5 cm loreit. unterseits rauls, coberseits glatt. Blatt-

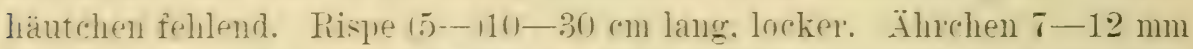
linge. meistens drei- l,is fünflulitig. Entere Hüllspelze eiförmig-länglich.

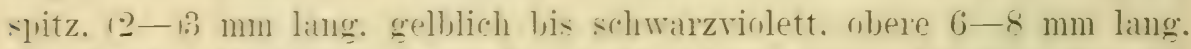
lanzettlich. Derkinelzen fast lineal. lis $11 \mathrm{~mm}$ lang. Älrchenachise mit bis $10 \mathrm{~mm}$ langen. Weiforn Härrhen. Blüte .Juli bis August bis Oktoberl.

An Ufern und in Sümpfen, zuweilen in angrenzende Formationen

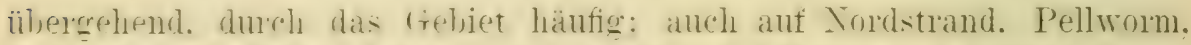
Füln. Amrun. sylt und Föm. sowie seltener auf Hogge und ifrühery Helgoland (Hallier, Dalla Torre; jetzt verschrunden).

Weber nennt als erster diese Art (Prim. Fl. Hols. 10 [1780]).

\section{Formen.}

1. Alle Stengel aufrecht:

f. genuinu A. 1. Gr. Syn. Mitt.-Europ. Fl. II. 1. 330 (1900). Häufig.

Dazu gehören:

f. muila G. F. W. Meyer Hannör. Mag. Stück 22. 169 (1824). - Pflanze 0,15-0,4m hoch; Stengel oft zahlreich genähert, fast rasig. - Z. B. am Elbufer mehrfach!!, ferner Kiel: am Barsheker See! und Schwansen: am Schwansener See! (A. Christiansen); Sylt und Amrum!!. 
f. subuniflora DC. Fl. de France V. 263 (1815). - Stengel niedrig; Rispe armährig; Ährchen meistens einblütig. Z. B. Hamburg: Steilshop!!. Kiel: Wellingdorf und Rathmannsdorf (A. Christiansen!). Föhr: Goting und Witsum!!. Amrum: Norddorf!!. Sylt: am MLorsumkliff!! - Zuweilen kombiniert mit folgender Form.

- Stengel z. 'T'. ausläuferartig, liegend, an den Knoten oft wurgelnd: f. stolonifera G. F. W. Neyer a. a. O. 171 (1824).

Auf Sandboden, besonders an Ufern, zerstrent, stellenweise in Menge. - Hamburg: am Elbufer von Lauenburg abwärts verbreitet (Sonder usw.)!!. La uenburg: Güster, am Elbe-Trave-Kanal!!. Kiel: zwischen Friedrichsort und Bülk (Nolte!), Holtenan (Lange!) und sonst mehrfach (A. ('hristiansen!). Eckernförde (Prahl)!!. Angeln: zwischen Maasholm und Öhe (Nolte!, Hansen!), Düttebüll (Lund), an der Flensburger Föhrde (Hansen, Hb. Prahl!), am Sïdensee (Prahl). A penrade (Lange!)!!. Husum: Schobüll (F. r. Hüller). Föhr (Nolte!): Wyk!!. Sylt: List, Morsum, Eidumer Vogelkoje (Jaap); mehrfach!!. Amrum (Jessen!): Norddorf!!. Röm: im Osten nicht selten (Jaap).

\section{Mißbildungen.}

f. m. strintipirtn Reichenb. Fl. San. $13(1842)$ - - Blätter weifgestreift. Sylt: am Morsum-Klift!! Röm: Torfmoor bei Twismark (Jaap).

36. Gattung.

\section{Molinia.}

Schrank Bayr. Fl. I. 334 (1789).

97. (sp). 36). Molinia coerulea Moench Meth. 1s:3) (179)

4. Grundachse dichtrasig, mit kurzen Ausläufern. Stengel $(0,1-$ $0 . t-11-1.51 \mathrm{~m}$ hoeh, aufrecht. g]att, in der oberen Hälfte blattlos. Blattscheiden eng, glatt. Blattläche $2-10 \mathrm{~mm}$ breit. unterseits glatt. oberseits rauh, am firunde wimperhatarig. Blatthäutchen kurz, zerrisien. Rispe 11-1.5-35 c'm lang. locker oder znsammengezogen. Ährehen lang. mit 11-13-6 Blüten, seitlich zusammengedrǘdit. Lntere Hüllspelze spitz, eimnervig, obere breiter, ein- oder dreinervig, etwa $2 \mathrm{~mm}$ lang: Deckspelzen 4-5ィ-6) mm ling. fünfnervig. stumpflich. Ährehenachse kahl. Blïte Juli bis Oktober. 
Auf torfigem Boden der Mnore. Sïmpfe, Wiesen. Wälder ust. häufig, auch auf Föhr, Amrum, Sylt und Röm.

Hierher zählt vielleicht Kryllings ITiridarim Danic.65) [1688]) ..Gramen spica niogra. Swartz-(iras" von Llstrup und Gramm im Kreise Hader's-

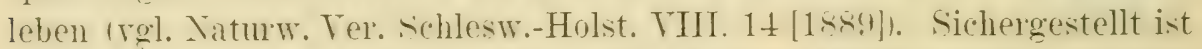
sie für Holstein durch Wober (Prim. Fl. Hols. $7[1780]$ als Airm romentert.

\section{Formen.}

1. Ährchen zwei- bis fünfblïtig, an ziemlich derben Ästen; Blätter

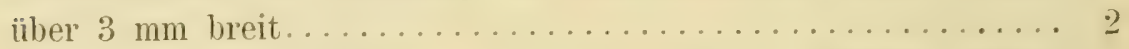

- Ährchen ein- bis dreiblütig, an haarfeinen Ästen; Blätter schmal:

f. capillaris Rostrup in Lange Haandb. Dansk. Fl. 4. Udg: 69 (1886).

Schleswig (Lange): Röm: zwischen Kongsmark und Lakolk (Prahl 1905!).

2. Stengel $(0,1-) 0,3-0,6(-0,8) \mathrm{m}$ hoch; Blätter $3-6 \mathrm{~mm}$ breit... 3

- Stengel $(0,6-) 1-1,5 \mathrm{~m}$ hoch, sehr fest; Blätter $6-10 \mathrm{~mm}$ breit 5

3. Stengel mehrmals länger als die Blätter, meistens $0,3-0,6 \mathrm{~m}$ hoch +

- Stengel nicht oder wenig länger als die Blätter, etwa $0,1 \mathrm{~m}$ hoch:

f. depauperate A. u. Gr. Syn. Mitt.-Europ. Fl. II. 1. 337 (1900). - Rasen klein, mit wenigen Stengeln und Blatttrieben; Rispe kurz, zuweilen kopfig; Ährchen kurzgestielt, wenig zahlreich.

Lauenburg: zwischen Bröthen und Wendisch-Lieps (J. Schmidt!). Hamburg: Rotenhaus (J. A. Schmiad 1865!), am Bramfelder Teich (A. Junge!), Eppendorfer Moor!!. Lübeck: Priwall (Häcker!). Fïhr: Hedehusum!!. Amrum: bei Nebel und Norddorf mehrfach!!. Sylt (F. v. Müller): Morsumheide!!. Röm (Reinke 1902!): westlich von Twismark (J. Schmidt 1905!).

4. Ährchen violettbraun bis graublau; Rispe dichter:

f. gemina A. u. Gr. a. a. O. II. 1. 337 (1900). - Häufig. - Ährchen grünlich, zuweilen gelblich; Rispe lockerer:

f. viridiflora Lejeune Rer. Fl. Spa 16 (1824). - Nicht selten. -

Übergangsformen nach $f$. gemina sind sehr verbreitet.

5. Rispenäste sämtlich kürzer bis wenig länger als die Achsenglieder . . .

- Rispenäste wenigstens zum Teile mehrmals länger als die Achsen-

6. Rispe groß, dicht; Rispenäste etwa so lang oder etwas länger als die Achsenglieder, reichährig; Ährchen genähert:

f. rolusta Prahl Krit. Fl. Schlesw.-Holst. 257 (1890). 
Auf aufgebrochenem Heide- und Moorboden hin und wieder, zuweilen in Menge; zuerst 'T'ondern: Leck (Prahl 1889!).

- Rispe schmal, unterbrochen; Rispenäste kïzer (zuweilen mehrmals) als die Achsenglieder, mit wenigen Ährchen:

f. subspicuta Figert in Fiek und Schube Ber. Schles. Ges. 70. II. 88 (1893).

Auf trockenem Moor- und Sandboden zerstreut, stellenweise reichlich; oft kombiniert mit $f$. genuina.

7. f. armdinacea Ascherson Fl. Brandenb. I. 837 (1864). - Pflanze bis 1,5 m hoch; Blätter $8-10 \mathrm{~mm}$ breit.

An Ufern (und in Wäldern) nicht selten.

Hierher gehören: M. silvestris Schldl. in Sonder Fl. Hamb. 56 (1851), 1I. coerulea f. major Hornemann Dansk. Oec. Plantel. 3. Udg. 1. 99 (1821) (wahrscheinlich) sowie $f$. maior Langmann Fl. Mecklenb. 89 (1841).

\section{Mißbildungen.}

f. m. vivipara G. F. WT. Meyer Chloris Hamnor. 626 (1836). - Ährchen in Laubsprosse auswachsend. - Stormaln: am Helkenteich bei Trittau!!, Bramfelder Teich (C. Timm). Hamburg: Eppendorfer Moor (J. A. Schmidt 1865!), Borstler Moor (C. Timm 1881!).

\section{Gattung.}

\section{Diplachne.}

Pal. Beaur. Agrost. 80 (1812).

98. (adr. 54). Diplachne fusca Pal. Beaux. Agrost. $16331812 \%$

7. Arundachse dichtrasig, zumeilen mit kmzen Ausläutern. stengel (1.3-0.S m hoch, aufrecht oder aufsteigend, glatt. Blattseheiden slatt. weit herab otfen. Blattlache in der Regel getialtet. rauh. Blatthäutchen (1-) $2-4 \mathrm{~mm}$ lang. Rispe $10-20 \mathrm{~cm}$ lang, etwas zusammengezogen. ت̈hrehen schmallanzettlich. 1--1.8 cm lang. bis zehnblütig. Hüllspelzen mgleich, stmmptlich, cinnervig. untere bis 2.5 , ohere his 4 mm lang. Deckspelzen bis $6 \mathrm{~mm}$ lang. spitzlich, mit Mittelner und zrei Randnerven: Verven muterwäts dicht liuz weibharig. Alnchenachse unter den Blüten kurz behaart, sonst kahl. Bliite August bis Oktober.

Auf Schutt bei Hamburg selten.

Hamburg: bei der Wollkämmerei am Reiherstieg l.J.sehmidt 1894!.

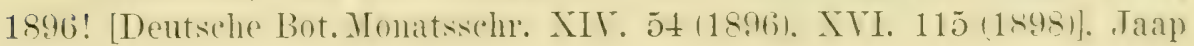
$1897 !)$ 


\section{Gattung.}

\section{Melica.}

L. Gen. plant. ed. 5. 31 (1754).

1. Ährchen mit zwei Zwitterblüten; Ährchenstiele oberwärts kurz behaart...... 2

- Ährchen mit einer Zwitterblüte; Ährchenstiele kahl .............. uniflora.

2. Ährchen zu vielen in dichter, ährenartiger Rispe.............. altissima.

- Ährchen in lockerer, höchstens unterwärts rispiger, wenigähriger Traube.

M. nutans.

99. (adv. 55). Melica altissima L. Spec. plant. ed. 1. 66 (1753).

7. Grundachse lockerrasig. kriechend. Stengel meistens aufrecht, 0,6-1,2 m hoch, rauh. Blattscheiden eng, rauh. Blattfläche bis $2 \mathrm{~cm}$ breit, beiderseits rauh. Blatthäntchen stumpf, bis 5 mm lang. Rispe ährenförmig, dicht oder am Grumde unterbrochen, 10-20 cm lang. Ährchen bis $8 \mathrm{~mm}$ lang, an behaarten Stielen, mit zwei Zwitterblüten. Hüllspelzen länglich, stumpf, dentlich umgleich. Deckspelzen kahl, stumpf, stachelspitzig, bis $7 \mathrm{~mm}$ lang. Blïte Juli.

Auf Schutt bei Hamburg selten.

Hamburg: in einer Gartenhecke in Flottbek.(C. Timm), beim Altonaer neuen Begräbnisplatz (Zimpel 1892!); ferrer auch auf Schutt im Botanischen Garten (Th. Meyer 1891!).

100. (s). 37). Melica nutans L. Spec. plant. e(l. 1. 66 (175:3).

7. Grundachse lockerrasig. lang kriechend. Stengel anfrecht oder aufsteigend, dïm, glatt oder schwach rauh, 0.2-0.6 m hoch. Blattscheiden stark rückwärts ramh. Blattflache 3-5 mm breit, unterseits glatt, oberseits mäßig rauh, an den Rändern stark rauh. Blatthäutchen fehlend oder ganz kurz. Rispe 5-8 cm lang, traubig oder unterwärts rispig. Ährchen 6-7 mm lang, mit zwei Zwitterblüten, an oberwärts kurz behaarten Sticlen. Hüllspelzen stumptlich, einnervig, etwas moleich, fast so lang wie das Ährchen. Deckspelzen bis $7 \mathrm{~mm}$ lang, sieben- bis neun- (bis elf-mervig, stumpflich, kahl. Ansatz zur dritten Blüte länglich-dreieckig. Bliite Mai bis Juni (und Juli).

In Täldern und Gebüschen im südöstlichen Gebiet zerstreut, nordwärts seltener bis Flensburg, im Westen nur bei Husum.

Lauenburg: an Abhängen am Schallsee bei Techin, Lassahn und Hakendorf!!, bei Ratzeburg (Nolte 1820!. Häcker!) zwischen Dermin und Bäck!! sowie nach Farchau zu (J. Schmidt 1888, Prahl 1903!). Möln (1 aban!), Grambek (Zimpel 1894!, 1896!), Breitenfelde (J. Schmidt 1911)!!, 
beim sandkrug (Clandius), im sachsenwalde ron Friedrichstuh bis zu

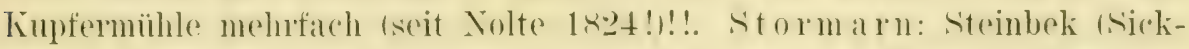

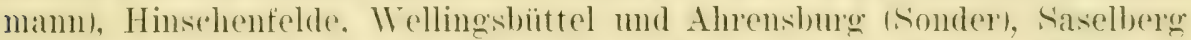

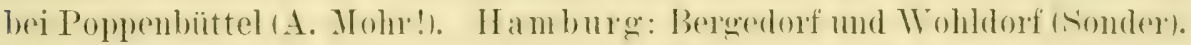

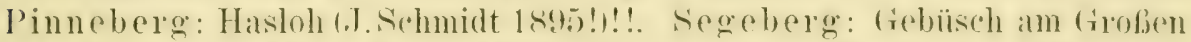

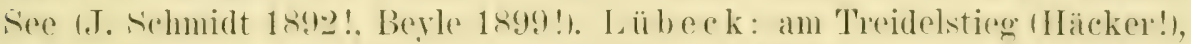

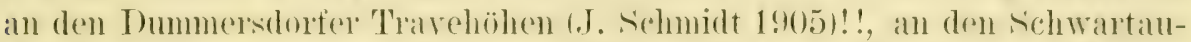
Abhängen westlich von Ratekan 1908!.. Futin: Gichölz am Kleinen

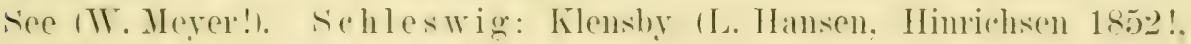
1877!, W. Hansen 1887!). Flensburg: am Wall am alten Wege nach der Kuptermühle L. Hamsen 1s4t!). Husum: Engelsburg 1. ('hristiansen!).

Wer die Art zuerst im Gebiete beobachtet hat, ist nicht festzustellen, da die ältesten Angaben infolge von Verwechslungen mit $\boldsymbol{M}$. uniflora und beim Fehlen von Belegen unsicher sind. Es ist anzunehmen, daß Aira nutans Weber (Prim. Fl. Hols. 7 [1780]) nicht hierher, sondern zu der viel häufigeren $\boldsymbol{V}$. uniflora zu zählen ist, die in Webers Aufzählung fehlt. Beide finden sich nebeneinander genamnt bei Buek (Hoppe Bot. Taschenb. 91 [1801] für Hamburg; von hier notiert auch IIößler das Gras (Handb. Gewächsk. 107 [1815]), und zwar für Eimsbüttel, während er M. uniflora nicht von Hamburg erwäht. Für das damalige Dänemark hat M. Vahl den ersten Vermerk, trwïhnt aber unser Gebiet nicht (Flora Danica Heft 17 t. 962 [1790]).

Sichergestellt ist die Art durch Belege von Nolte. Nanche Angaben späterer Zeit beruhen auf Verwechslung; für manche fehlen wieder die Belege. Für Eutin sąt Lienau (Pflanzen Fürstent. Lüb. 5 [1863]) : „Häufig in den Holzungen." Diese Notiz kamn nur für M. uniflora gelten. Knuth nennt Lienau als Gewährsmann für die Orte: Beutiner Holz und Scharbeutz (Fl. v. Schlesw.-Holst. 766 [1887]) mit Unrecht. Bei Neumünster fehlt M. nutans, trotzdem Kirmis das Brachenfelder Holz als Fundort angibt (Progr. Realsch. Neumünster 11 [1883]). Die von Hennings (Naturw. Ver. Schlesw.-Holst. II. 1. ¿03 [1876]) übernommene Bemerkung für Bordesholn: in Holzungen (Amt Bordeshohı 39 [1842]) stammt von Nolte; auf sie geht der Hinweis für Kiel (Knuth Schulfl. v. Schlesw.-Hulst. 368 [1887]) zuriek. Dem Elsdorfer Gehege bei Rendshurg (Lienau nach Handschriftl. Verz.; Knuth a. a. 0. 766 [1887]) fehlt M. nutans (vgl. $f$. autumnalis bei folgender Art). Von Hohenhain und Delve in Dithmarschen hat Grünwald das Gras notiert. Wenn Knuth (a. a. 0. 766 [1887]) das Lauerholz bei Lübeck erwïhnt, als Finder aber Hansen, so dürfte er Häckersche Pflanzen vom Treidelstieg gesehen haben, welche Hansen mit Aufschrift Lauerholz versehen hatte. Eine Angabe für Segeberg: Pronstorf (Naturw. Ver. Schlesw.-Holst. III. 100 [1878]) ist nach J. Schmidt zu streichen.

\section{Formen.}

f. mumiculutu Borbas Oestr. Bot. Zeitschr. XXXIII. 275 11883\%. - Lutere Rispenäste mit einem grundständigen Zweige.

$$
\text { Selten. - La u e n burg: Techin!!. }
$$

101. (sp. 38). Melica uniflora Retzius Observ. Bot. I. 10 11779).

4. Grundachse lockerrasig. kurz kriechend. Stengel aufrecht oder aufsteigend, sehr dümn. meistens glatt, $0.3-0,6 \mathrm{~m}$ hoch. Blattscheiden 
eng, rückwärts rauh. Blattfläche 12-13-4 mm breit, rauh, zuweilen schwach behaart. Blatthäntchen fast fehlend, gegenüber der Blattfläche mit spiłzlichem Anhängsel. Rispe sehr locker, mit verlängerten, verzweigten Ästen. Ährchen כ̆-(j) mm lang. mit einer Zwitterblüte, an kahlen Stielen, aufrecht. Hüllspelzen כ̃ mm lang, etwas ungleich, spitzlich. Deckspelze grünlich. sicbennervig; stumpf. Ansatz zur zweiten Blüte länglich. Blüte Mai bis Juni, selten später.

In Wäldern und Gebüschen hesonders auf tonigem Boden; im Osten häufig, im Westen weniger verbreitet; fehlt auf den Nordseeinseln.

Hierher wohl Aira nutans Weber (Prim. Fl. Hols. 7 [1780]). Von Nolte genannt Nov. Fl. Hols. 12 (1826).

\section{Formen.}

Tom Typus weichen ab:

f. autumnalis A. u. Gr. Syn. Mitt.-Europ. Fl. II. 1. 353 (1900). Pflanzen 0,2--0,5 m hoch. Rispenäste aufrecht zusammengezogen. Ährchen 4-5 mm lang. - In der Tracht M. mutans ähnlich. - Bliite Juli bis September.

Selten. - Rends burg: Gehege Osterhamm(A.Christiansen 1912!). - Eine Annäherungsform scheint eine Pflanze von Eckernförde: Gehege Dornbrook und großes Gehege bei Wittensee (A. Christiansen!) zu sein.

f. deparperata A. Christiansen nov. f. - Stengel sehr fein, fadendünn, mit nur ein bis zwei Ährchen.

Kiel: Rastorfer Mühle (A. Christiansen!).

39. Gattung.

Koeleria.

Persoon Syn. I. 97 (1805).

1. Stengel aus derber Grundachse aufrecht bis aufsteigend; Pflanze 4 , mit nicht blühenden Sprossen; Deckspelze spitz bis stumpf .................. 2

- Stengel aufrecht; Pflanze $\odot$, ohne nicht blühende Sprosse; Deckspelze zwischen zwei Seitenspitzen stachelspitzig oder kurz begrannt................

2. Deckspelze stumpf; Stengel am Grunde von den bleibenden Resten alter Blattscheiden zwiebelartig verdickt.................................

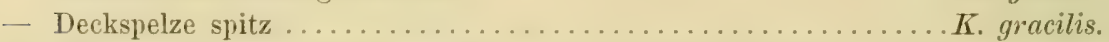

3. Hüllspelzen behaart; Deckspelze aus dem Einschnitt mit bis $2 \mathrm{~mm}$ langer

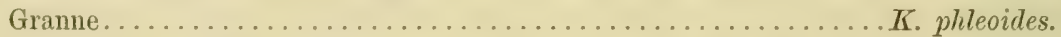

- Hüllspelzen kahl; Deckspelze im Einschnitt stachelspitzig .....K. obtusiflora. 
102. (adv. 5(i). Koeleria gracilis I'ers. Yyn. I. 9711805$)$ (2lW.

7. Grundachse dichtrasig. Stengel aufrecht, $(0,1-) 0,3-0,4 \mathrm{~m}$ hoch, in dere Regel kahl. gratt, dium, am Grumble nicht verdickt. Blattscheiden sämtlich oder nur an den Grumdblittern zerstrent bis dicht kurzhatrig. Blattlaiche der Grumdbläter oft gefaltet, 11-3) mun breit, der Stengelblatter meistens flaroh, bis 2 mm breit, fein kurhanrig. Blatthäutchen fast fehlend. Rispe bis 10 ('m lang, schmal bis breitzylindrisch, meln oder weniger dicht. Ährchen gedrängt, 4-5 mm lang, zwei- bis drei(bis vier-bblitig. Hülspelzen glatt, kahl, etwas mgleich, spitz. I)eckspelzen in der Regel kahl, spitz, bis $4 \mathrm{~mm}$ lang. Blüte Mai bis August.

Auf Schutt und Grasland selten.

Hamburg: Mühlenkamp in Winterhude (Laban 1874!), bei der Wandsbeker Dampfmühle mehrach (rimpel 1895-1901!), in Booth' Garten in Flottbek (C. Timm 1886, W. Hansen 1891!, Laban 1896!). Kiel: am Mönkeberger Moor (A. Christiunsen 190s!). Tondern: Lïgumkloster (Seehusen 1861!; Ho. Kopenhagen).

Nach Roeper (zur Fl. Mecklenb. 199 [1843]) soll K. cristata (die allerdings nicht in ganzem Umfange mit $K$. gracilis identisch ist, aber früher für unsere Gegend genannt wurde) in allen Nachbarländern Mecklenburgs, zu denen Lübeck, Holstein und Hamburg. gerechnet werden, beobachtet worden sein. Es ist nicht festzustellen, worauf sich diese Angabe gründet.

103. (sp). 39). Koeleria glauca De Candolle Catalog. Hort. Monsw. 116 (1813).

4. Grundachse dicht bis locker bis kurz kriechend. Stengel am Grunde von den sich überdeckenden, zuletzt in Fasern zerfallenden alten Blattscheiden zwiebelartig rerdickt, $10,1-00.3-0,6 \mathrm{~m}$ hoch. anfrecht. in der Regel tein behart. Blätter grangrün. Blattscheiden meistens dicht behalart bis (die oberen) kahl. Blattfläche schmal. oft gefaltet, fein behart. Blatthäutehen kurz, zuweilen bis $1 \mathrm{~mm}$ lang. Rispe meistens zỵlindriseh. dicht bis etwas locker, am Grunde öfter unterbrochen. Ährchen $4-5 \mathrm{~mm}$ lang, zwei- bis drei- (bis rier-blütig. Hüllspelzen etwas mngleich, meistens stumpf, selten spitzlich, meistens kahl. Deckspelzen stumpf, kalhl. Blüte Juni bis August.

Zerstreut anf Sandfeldern und an sandigen Abhängen im südöstlichen Gebiet bis Hamburg-Lübeck, selten auf Dünen der Insel Röm.

- Iröglicherweise gehört Pon eristutn Weber (Prim. Fl. Hols. 7 [17-0]) hierher, doch spricht dagegen, dal. Nolte $K$. ylumer unter der Bezeichnmor Aira glance als neu für das besprochene Gebiet (Holstein) aufzählt (Nov, Fl. Hols. 12 [1826]). 


\section{Formen.}

1. vav. typica Domin Bibl. Bot. 65. 5̄ (1907). - Grundachse wenig restrerkt, mit $0.3-0.6 \mathrm{~m}$ hohen stengeln: Blattscheiden und -flächen wenigstens zum Teile behaart; Blattfläche wenig fest.

Nur im südöstlichen Gebiet. - Von L a u en burg bis Stormarn: Boberg längs der Elbhöhen hin und wierler seit Nolte!)!.. Lübeck: frönaner Heide ILuther nach Klattı, sibllich von Falkenhusen (J. Schmidt 1895!).

Die Rasse zerfällt:

f. gemina Domin a. a. 0. 56 (1907). - Rispe dicht, nur zuweilen am Grunde wenig unterbrochen. - Normale Form.

f. Tobate Marsson Fl. v. Neuvolpommern usw. б73 (18199). -

Rispe locker, besonders am Grunde unterbrochen, mit bis $5 \mathrm{~cm}$ langen Ästen. - Hamburg: Geesthacht Nolte 1821!, J. A. Schmidt!), Besenhorst (Nolte 1824!). Stormarn: Ladenbek (J. A. Schmidt 1873!, Beyle 1891!), Boberg (J. Schmidt!).

- var. intermedia Domin Bot. Tidsskr. Bd. 27. Haefte 2. 221 (1906). stengel aus gestreckter. schrägr aufsteigender (irmanchse 0,1-0,2 m horch: Blattscheiden und -flächen kahl: Blattfäche kurz, starr, oft fast stechend. Stengel oft nur mit einem Blatte, dessen Scheide deutlich aufgeblasen ist. Rispe dirht, schmal zylindrisch, 11- 2-4 cm lang.

So auf Dïnen der Insel Rö m (L. Hansen!): in der Südhälfte der Insel vielfach $1902,1904 !$ !.

Dazu :

f. abrreviata J. Schmidt Allg. Bot. Zeitschr. XIII. 25 (1907). Rispe kopfig, 10-15̃(-20) mm lang. — R ö m (J. Schmidt!).

Die erste der beiden Varietäten liegt im Herb. J. J. Meyer (Altona) von „Itzehoe $1817^{*}$ !. Die Pflanze ist aber wahrscheinlich nicht bei Itzehoe gesammelt worden; der Besitzer hat sie während seines Aufenthaltes in der genaunten Stadt von einer anderen Ortlichkeit erhalten. und zwar in Jahre 1817. Eine genauere Standortshezeichnung fehlt bei einer K. glauca im Kopenhagener Herbar ron Lübeck (leg. Kjellberg 1901 !).

Im Herb. Hinrichsen (Altona) liegt eine angeblich 1853! auf Röm gesammelte Ptlanze der var. intermedia, deren Grundblattscheiden dicht kurzlaarig sind und dadurch auf den Typus hinweisen. Sie wäre als $f$. hirta zu bezeichnen.

104. (adv. 57). Koeleria phleoides Pers. Syn. I. (17 (1805).

¿. Stengel zu mehreren bis vielen, aufrecht oder aufsteigend, $5-20 \mathrm{~cm}$ lockl, meistens einfach. kahl. Blattscheiden eng, in der Regel zerstreut behaart: Blattfläche behaart, am Rande gewimpert. Blatthäutchen geteilt, 2-3 mm lang. Rispe dicht oder etwas locker. meistens $1-4 \mathrm{~cm}$ lang; Ährchen 13-14--5 mm lang, drei- bis fünflulütig. Hüllspelzen spitz, ungleich, 
untere bis $3 \mathrm{~mm}$ lang, cimmervig. obere bis 4 mm lang. dreinervig. zerstrent behaart. Deckspelzen behast. an der spitze zweizahnig. ans dem Einschnitt mit bis $2 \mathrm{~mm}$ langer Granne. Blüte Mai bis Juni.

Auf Schutt bei Hamburg und Kiel selten.

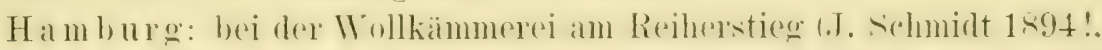

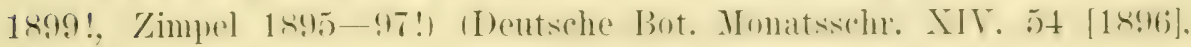
XVT. 115 [1898]). Kiel: Neumühlen (A. Christiansen 1909!).

Domin.sagt (a. a. 0. 262 [1907]) für Hamburg: "locis pluribus introducta", doch ist das Gras nur an einem Orte gesammelt worden, hier allerdings jahrelang. Die nachfolgend genainte Form sammelte Zimpel und gab sie als $f$. condensata an (Deutsche Bot. Monatsschr. XIV. อ̃t [1896]); ein Autorname fehlt, doch ist wohl zweifellos $K$. condensata Boiss. et Blanche $=K$. phleoides var. condensata Boiss. Diagn. Ser. II. 4. 134 (1859) gemeint; diese Koeleria ist indessen nach Domin (a. a. 0.274 [1907]) zu K. obtusiflora Boiss. zu ziehen, die bei Hamburg gesammelte Abart aber zur f. pumila zu stellen.

\section{Formen.}

Tom Typus weicht ab:

f. pumila Ledebour FI. Ross. IV. 403 (1853) z. T. - Stengel aufrecht, oft einzeln, bis $10 \mathrm{~cm}$ hoch. Rispe kurz- bis länglicheiförmig, dicht.

Hamburg: Wollkämmerei am Reiherstieg (Zimpel 1896!).

105. ladr. 58). Koeleria obtusiflora Boiss. Diagn. Ser. I. 7. 121 (1846).

$\odot$. Stengel zu mehreren, aufsteigend, $0,3-0,4 \mathrm{~m}$ hoch, glatt, kahl. Blattscheiden kahl oder die unteren behart. glatt. Blattläche $2-4 \mathrm{~mm}$ breit, oberseits schwach rauh und zerstrent behart, unterseits kahl. Blatthäutchen sehr ku\%. Risne dicht, zylindrisch, $3-4$ cm lang, bis 1,2 ('m breit. Ährchen 4 -5) mm lang, drei- bis tünfblütig. Hüllspelzen spitz. untere etwa $2.5 \mathrm{~mm}$, obere $3.5 \mathrm{~mm}$ lang. Deckipelzen bis 3.5 lang, fünfnervg, länglich, stumpf. zweizähnig. die der unteren Blïten zwischen den beiden Spritzen mit kurzer Stachelspitze. Kahl oder zerstrent kurzharig. Torspelze kürzer als die Deckspelze. Blüte .Juli bis August.

Auf Schutt bei Kiel selten.

Kiel: Neumïhlen (A. Christiansen 1909!).

\section{Gattung.}

\section{Eragrostis.}

Host Gram. Austr. IV. 14 (1809).

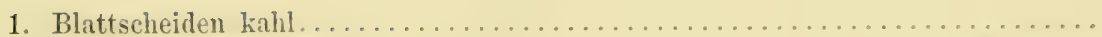




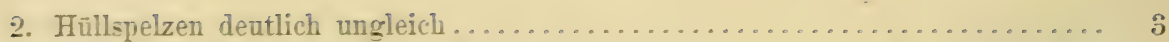

- Hüllspelzen fast gleich, etwa $2 \mathrm{~mm} \operatorname{lang} . . . \ldots . . . . . . .$. . megastachya.

3. Rispenäste glatt; Dechspelze mit schwachen Yerren.............. pilosa.

- Rispenäste rauh; Deckspelze mit drei starken Nerren .......E. caroliniana.

106. uadr. 5!1. Eragrostis megastachya Link ratil. Hoit. Berol. I. 187 (1827).

¿. strugel zu mehreren. meistens ans liegendem firunde aufsteigend. selten anfercht. $0.2-0.6 \mathrm{~m}$ lang. ijter aus den husten rerzweigt. glatt. Blattrideiden clatt. kahl. Blattllärhe 2-7 mu hreit. unterseits glatt.

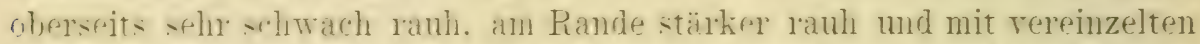
Haarerl. Blatthäutchen sshr kurz, jedprseits unit einem dichten Büsclel weifer Haare. Rinpe 5-15) (m lang. mit rom frunde ährchentragenden Ästen. Ährchen 5-12 mn lang. 10-25blütigr. Hüllspelzen fast gleich.

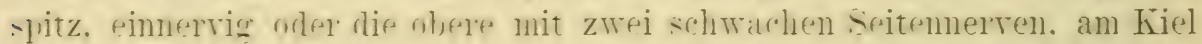
fein gewimnert. Derkelelzen 2-2.5 mm lang. dreinervig. stachelsitzig. riel länger als die Torspelze. Bliite Angust bis Oktober.

Auf Schutt bei Hamburg selten.

Hamburg: Hammerbrook (Laban 1865!, Hb. J. A. Schmidt), bei

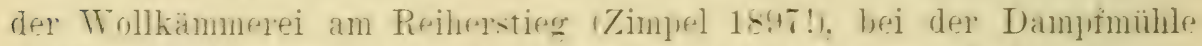

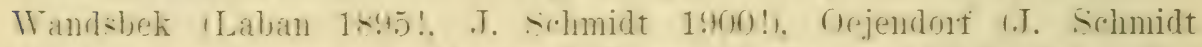

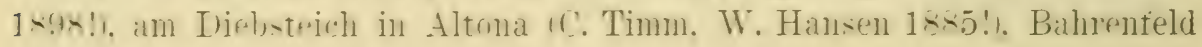
$1901 !$ !

Die Labanschen Spezimina aus dem Hammerbrook zeigen kleine, wenigblütige Ahrchen und erimern dadurch an die folgende Art, ron der E. megastachya rielleicht nur eine Rasse ist. Die kahlen Scheiden aber lassen die Pflanze als E. megastachya

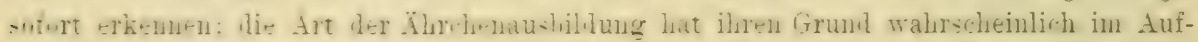
wachsen in dichten Pflanzenbestande. Derartige Formen sind $f$. cilianensis A. u. Gr., eine unwichtige Standortsform.

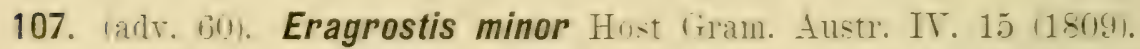

○. Stengel weniger zahlreich. am Grunde oft niederliegend, $0.2-0.5) \mathrm{m}$ lans. rit rerzweist. glatt. Blattcheiden glatt. unterwärts shwächer. oleerwärts stärher zerstreut langhaarig. Blattfläche 2-5 nm brwit. unterseits tast glatt. nilerseits stärer rauh. am Rande rauh und nach rem firunde zu mit einzehen Wimperhaaren. Blatthäutchen kurz. mit shwähren Harbuschehn. Rispe bis 15 cm lang. lockerer als bei

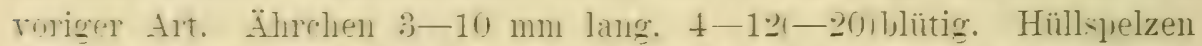
einnervig. slitz. untere $1 \mathrm{~mm}$, chlere fast $1.5 \mathrm{~mm}$ lang. am Kiele rauh.

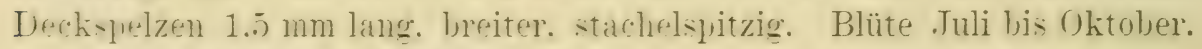

Auf Schutt bei Hamburg selten. 
Hamburg: auf Baggererde im Hammerbrook (Lablan 1 sijs)[mehrfach]!!, bei der Wandsbeker I) amp fmühle (.Jalp 1893!. Zimpel 1894!. .J. Sochmilt 18!5!, 1900!). Wollkitmmerei am Reiherstieg (Kittsch 1s!s!), Winterhude (Jaap!, Hb. Lübeck), Bahrenfeld 1900!!.

\section{Formen.}

f. robusta nov. f. - Pflanze kräftig; untere Rispenäste mit bis sechs grundstindigen Zweigen. - H a m burq: Bahrenteder Kiesgrnben 1905:!.

108. ladt. 61). Eragrostis pilosa Pal. Beaur. Agrost. 162 (181:).

$\odot$. Stengel zu mehreren, autsteigend oder am (irtunde niederliegend. $0.1-0.3 \mathrm{~m}$ hoch. glatt. Blattscheiden glatt, kahl. Blattfläche 1-3 mm breit, muterseits glatt. oberseits schwach rauh, an Rande ohne Haare und Drïsen. Blatthäutchen fiast fehlend, an jeder seite mit einem Büschel etwa $3 \mathrm{~mm}$ langer, weißer Haare. Rispe 5-10 cm lang, ihre unteren Äste mit bis fünf grundständigen Zweigen, alstehend. Äste glatt. Ährehen entfernt, 3--5 mm lang, rier- bis zehnblütig. Hüllsprelzen häutig, spitz. untere kaum $0,5 \mathrm{~mm}$. obere fast $1 \mathrm{~mm}$ lang. Deckspelzen stumpilich, dreinervig, etwa $1 \mathrm{~mm}$ lang. Blüte Juli bis September.

Auf Schutt bei Hamburg selten.

Hamburg: anf dem Königsland bei Wandsbek (.J. Schmidt. Progr. Unterr. Johamnis Hamb. 31 [1890]). auf dem Grasbrook (.J. Schmidt 1s!16!).

109. (adv. 62). Eragrostis caroliniana Scribner ILem. 'Torr. Bot. Club) T. 49 (1894).

$\odot$. Stengel zu mehreren, meistens aufsteigend, $0,1-0,4 \mathrm{~m}$ hoch. Blattscheiden glatt, kahl. Blattläche bis $3 \mathrm{~mm}$ breit. oft eingernllt. Blatthäutchen kurz, mit Wimperharen. Rispe $t-10 \mathrm{~cm}$ lang. schmal. ihre unteren Äste mit ein bis zwei grundständigen Zweigen. Äste rauh. Ährehen oft entfernt, an 2-5 mm langen Stielen, (2-) $4-7 \mathrm{~mm}$ lang, drei- bis sechs- (bis zwölt-blütig. Hüllspelzen umgleich, häutig. spitz. Deckspelzen dreinerrig. etwas über $1 \mathrm{~mm}$ lang. stumptlich. Blüte Juni bis Oktober.

Auf Schutt bei Hamburg selten.

H a m burg: bei der Wollkämmerei am Reiherstieg (.T.Sehmidt 1s96!). bei den Altonaer Wasserwerken bei Blankenese (Zimpel 1s91!. T. Schmidt 1893!), bei der Wandsbeker Dampfmühle (J. Schmidt 1897!).

$\mathrm{Zu}$ dieser Art zählen E. suaveolens Deutsche Bot. Mlonatsschr. XIV. 53 (1896). E. abessinica a. a. O. XIV. 54 (1896), E. lanceolatus a. a. O. XVII. 125 (1899), nicht Roth, und E. ? laxus a. ล. O. XVII. 12 อ̆ (1899), nicht Hornemann. 


\section{Gattung.}

\section{Schismus.}

Pal. Beauv, Agrost. 73 Pl. XV. fig. IV (1812).

1. Deckspelze mit zwei stumpflichen Seitenzähnen, ihr Einschnitt etwa $1 / 5$ so lang wie die Spelze; Vorspelze fast so lang wie die Deckspelze............ S. calycinus.

- Deckspelze mit zwei spitzen Seitenzähnen, ihr Einschnitt etwa $1 / 3$ so lang wie die Spelze; Vorspelze wenig mehr als halb so lang wie die Deckspelze...S. arabicus.

110. (adv. 63). Schismus calycinus Dur.-Jouve in Billot Annot. 289 (1855).

$\odot$. Stengel zu wenigen bis vielen, niederliegend-aufsteigend, bis $0,2 \mathrm{~m}$ hoch, fein, glatt, kahl. Blattscheiden schwach erweitert, glatt. Blattfläche bis $1 \mathrm{~mm}$ breit, rimnig, am Rande zerstreut kurz behaart. Blatthäutchen in eine Haarreihe aufgelöst. Rispe länglich, $1-2(-3) \mathrm{cm}$ lang, kurzästig. Ährchen $5-7 \mathrm{~mm}$ lang, vier- bis achtblütig. Hüllspelzen spitz, breit hautrandig, etwa $4-5) \mathrm{mm}$ lang, untere fünf- bis sieben-, obere dreinervig. Deckspelzen mehrnervig, etwa $2 \mathrm{~mm}$ lang, oberwärts stumpfzweizähnig, unterwärts kurz behart. Vorspelze fast so lang wie die Deckspelze. Blïte Juni bis Juli.

Auf Schutt bei Hamburg selten.

Hamburg: bei der Wollkämmerei am Reiherstieg (J. Schmidt!.) (Für Mittelemropa durch Zobel bei Robleben unweit Roflan festgestellt.)

111. (adv. 64). Schismus arahicus Nees Fl. Afr.-anstr. 422 (18+1).

$\odot$. Stengel zahlreich, aufsteigend oder aufrecht, $0,3-0,6 \mathrm{~m}$ hoch, glatt. Blattscheiden glatt, kahl, nicht oder schwach aufgeblasen. Blattfläche schmal, oft gefaltet oder eingerollt.'Batthäutchen fehlend, durch Wimperhaare ersetzt. Rispe $4 \mathrm{~cm}$ lang, ziemlich dicht. Ährchen bis $7 \mathrm{~mm}$ lang, drei- bis achtblütig. Hüllspelzen wenig ungleich, sehr spitz, untere fün- bis siebemnervig, obere dreinervig, bis $7 \mathrm{~mm}$ lang. Deckspelze reichlich $3 \mathrm{~mm}$ lang, tief zweizühnig, am Grunde rauhharig. Blüte August bis September.

Auf Schutt bei Hamburg selten.

Hamburg: Georgswärder (Zimpel 1893!, J. Schmidt 1895).

\section{Gattung.}

\section{Dactylis.}

L. Gen. plant. ed. 5. 32 (1754).

112. (s1). 40). Dactylis glomerata L. Spec. plant. ed. 1. 71 (1753).

4. Grundachse dichtrasig. Stengel meistens aufrecht, $0,2-1,1 \mathrm{~m}$ 
hoch, glatt. Blattscheiden meistens schwach rauh. Blattläro $3-81-101 \mathrm{~mm}$ breit, unterseits schwächer, oberseits stärker rauh bis fast glatt. Blatthäutchen 2-41-81mm lang, zerrisisen gezähnelt. Rispes 3-10 -16) cm lang. geknäuelt, dicht oder öfter locker. mit einzehn gestellten Ästen. Ährchen (5-16-71-10) mm lang, drei- bis fünf- (bis nem-b)lütig. Hüllspelzen ungleich, mtere eimnervig, bis $3 \mathrm{~mm}$ lang, obere dreinervig, bis $5 \mathrm{~mm}$ lang, am Kiele rauh, spitz. Deckspelzen bis $6,5 \mathrm{~mm}$ lang, lanzettlich. am Kiel mit starken Trimpern besetzt oder seltener ungewimpert. oberwärts plötzlich abgesetzt oder allmählich rerschmälert; spitze selten in eine bis über $2 \mathrm{~mm}$ lange tramne ausgezogen. Blüte Iai bis. Juli, öfter in geringer Zahl bis Oktober.

Auf Wiesen und Triften, an Wegrändern und Abhängen. in Wäldern und Gebüschen hänfig; auch auf Röm, Sylt, Amrum, Föhr. Pellworm, Nordstrand und Helgoland.

Weber nennt die Art zuerst aus dem (iebiet (Prim. Fl. Hols. s [17s0]).

\section{Rasse.}

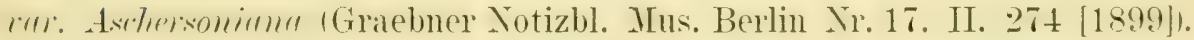
Grundachse lockenrasig. Stengel aufsteigend. Blattscheiden in der Regel kahl. Blattflache verlängert, schlafte Hüllspelzen fast völlig ungefärbt, häutig, wie die Deckspelzen am Kiel ungewimpert.

In schattigen Wäldern und Gebüschen, seltener an sonnigen Orten im östlichen Gebiete zerstreut bis stellenweise nicht selten, im mittleren Gebiete selten.

Hierher wahrscheinlich resp. sicher D. glomerata $\gamma$. lobata Drejer Fl. Hafn. excurs. 45 (1838) z. T., Lange Haandb. 4. Udg. 100 (1886) wenigstens z. T. und Prahl Krit. Fl. 257 (1890) z. T.; in allen drei Werken sind Ubergangsformen zum Typus inbegriffen. Über die Stellung der Rasse zum Typus der Art rgl. z. B. E. H. L. Krause in Bot. Centralbl. XXX. II. 120 ff. (1913).

\section{Formen.}

1. Rispe groß, deutlich gelappt .................... 2

- Rispe klein, dicht, ohne getremnte Knämel:

f. abbreciata Drejer Fl. Hafn. excurs. 44 (1838). - Pflanze niedrig, nur $0,2-0,3 \mathrm{~m}$ hoch; Blätter schmal.

Auf trockenem, dürrem Boden zerstrent, stellenteise nicht selten.

2. Blattscheiden kahl; Hüllspelzen nicht behaart........... 3

- Blattscheiden besonders die muterenl behart: Hüllspelzen ranhhaarig:

f. citiata Petermamn Fl. Lips. 80 (1838).

Zerstrent. - Pinneberg: Dockenhuden!!, Blankenese

(Deecke!). Fehmarn: Staberdorf!!. Oldenburg: Wandel- 
witz (J.Schmidt)!!, Kembs!!. Kiel mehrfach (A. Christiansen!). Angeln: Unewattholz!!. - Verbreitet sind Ubergangsformen, bei denen nur die Scheiden oder nur die Spelzen behaart sind; der Grad der Behaarung ist sehr ungleich. 3. Rispenäste und Ährchen nicht oder sehr schwach violett überlaufen: f. typica A. u. Gr. Syn. Mitt.-Europ. Fl. II. 1. 379 (1900). Häufigste Form.

Dazu als Unterformen:

f. multiflora G. Beck Fl. Nied.-Oesterr. I. 80 (1890). Ährchen acht- bis neunblütig. - Auf fruchtbarem Boden hin und wieder.

f. flavescens Schröter Deutsche Bot. Ges. X. 132 (1893). Ährchen gelbgrün. - Eine wenigstens als Übergang hierher gehörige Form beobachtete (mit „hellgrünen“" Spelzen) schon C. Timm bei Geesthacht (nach Knuth Fl. Schlesw.-Holst. 772 [1887]); derartige Formen sind auch später mehrfach gesammelt worden (! und !!).

- Rispenäste und Ährchen (wenigstens einseitig) lebhaft violett:

f. maritima Hallier Bot. Zeit. XXI. Beilage 7 (1863). — Ganze Pflanze bläulich-grün; Halm sehr stark und oft mannshoch, an jedem Knnten knieförmig gebogen; Rispenäste selır dick und steif, unterste 6-8 Zoll lang, zu zwei bis drei zusammengestellt, dunkelviolett (besonders oberseits); Spelzen an der nach oben gewandten Seite dunkelviolett, unterseits hell meergrün.

Helgoland: am Ostabhang auf Felsgeröll (Hallier, Brody!).

\section{Mißbildungen.}

f. m. viviparce Lange Haandb. Dansk. Fl. 4. Udg. 100 (1886). - Ährchen in Laubsprosse auswachsend. - Lauenburg: Ratzeburg (Zimpel 1899!). Hamburg: Tatenberg (Zimpel 1893!), am Diebsteich in Altona (C.Timm), Uhlenhorst (Kiusch!). Pinneberg: Flottbek (Laban!), Blankenese (Prahl!). St tormarn: Alt-Fresenburq (Friedrich!), Franenholz!!. Kiel: Gaarden (Hennings!), Ellerbek (A. Christiansen!). Eckernförde: Ascheffel (A. Christiansen!). Angeln: Ellenberg. (J. Schmidt!), Ulstrup!!. Had ersleben: Viktoriabad (A. Christiansen!). Helgoland (Kuckuck 1906!).

Die Pflanze des vorletzten Standortes ist besonders auffällig. Aus den Ährchen wachsen Laubsprosse aus, welche an ihrer Spitze kleine, dichte, fast kugelige Rispen tragen; die Sprosse sind beblättert. Es trägt also die Pflanze in ihrer Rispe zahlreiche kleine, vollständige Pflänzchen. 
f. m. Bractenta A. Christiansen nov. f. - Rispe am Grunde des unteren Astes mit kurem, laubigem T'Tagblatt. - Eutin: am Kellersee! und Preet\%: bei Wahlstorf! (A. Christiansen).

f. m. ramifere A. Christiansen nov. f. - Stengel aus einem oberen Knoten beästet. - Kiel: Kronsburg (A. Christiansen!).

43. Gattung.

\section{Sclerochloa.}

Pal. Beauv, Agrost. 98 (1812).

1. Untere Hüllspelze ein-, obere dreinervig................... procumbens.

- Untere Hüllspelze drei-, obere sieben- bis neunnervig............... dura.

113. ladv. (50). Sclerochloa dura P. Beaur. Agrost. 177 (1812).

$\odot$. Stengel einzeln oder zu mehreren, meistens aufrecht, $4-15 \mathrm{~cm}$ horeh, glatt. Blattseheiden glatt, gekielt. Blattläche o-t mm breit, am Rande rauh, kahl. Blatthäutehen bis $2 \mathrm{~mm}$ lang, spitzlich. Rispe 1-3 cm lang, dicht, ährenförmig. Ährehen zweizeilig gestellt, 6 drei- bis sechsblütig. Hüllspelzen sehr ungleich, untere 2 mm lang, dreinervig. obere 4 mm lang, sieben- bis neumervig. Deckipelzen bis $5 \mathrm{~mm}$ lang, fünf- bis siebennervig. knorpelig, glänzend. Blüte .Juni bis .Juli.

Auf Schutt bei Hamburg selten.

Hamburg: bei der Wandsbeker Dampfmühle (Jaap nach A. u. Gr. Syn. II. 1. $385[1900])$.

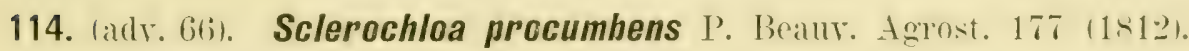

$\odot$. Stengel zu mehreren, oft niedergestreckt, $5-15 \mathrm{~cm}$ hoch, glatt. Blattscheiden glatt. gekielt. Blattlliche $2-3 \mathrm{~mm}$ breit. unterseits glatt. oberseits und am Rande wenig rauh. Bhatthäutchen bis :3 mm lang. spitzlich. Rispe 2-6 cm lang, mit kurzen Ästen, etwas locker. Ährchen bis $6 \mathrm{~mm}$ lang. drei- bis füntblïtig. Hüllspelzen moleich. untere otwa 2 mm lang. einnervig, obere :3 mm lang. dreinerig. Ineckspelzen his + mm lang. fünfnervig. Blüte Juli bis August.

Auf Baggererde bei Hamburg selten.

Hamburg: „Auf grasreichen 'Triften im Hammerbrook sehr vereinzelt" (J. A. Schmidt, 24. 8. 1866!).

Im Herbar des Botanischen Museums in Hamburg fand sich ein sehr beschädigtes, aber doch zu erkennendes Exemplar der Art, anscheinend auf Baggererde gesammelt. Die Pflanze wurde sonst in Norddeutschland früher bei Rostock! und Wolgast beobachtet. 


\section{Gattung.}

\section{Poa.}

L. Gen. plant. ed. 5. 31 (1754) z. T.

1. Untere Hüllspelze ein-, obere dreinerrig ..................... 2

- Beide Hüllspelzen dreinervig .......................... 6

2. Pflanze $\odot$; Deckspelzen undeutlich fünfnerrig $\ldots \ldots \ldots \ldots \ldots \ldots \ldots \ldots \ldots . \ldots \ldots$

- Pflanze 4; Deckspelzen mit fünf starken Nerven ................. 4

3. Hüllspelzen stumpflich, 1,5 und $2,5 \mathrm{~mm}$ lang; Rispenäste mit bis vier grund-

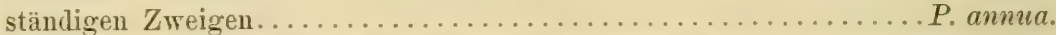

- Hüllspelzen spitz, 1,5 und $2 \mathrm{~mm}$ lang; Rispenäste mit sechs und mehr grund-

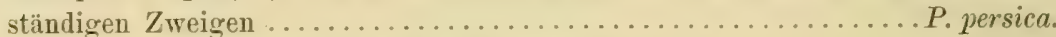

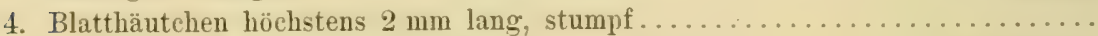

- Blatthäutchen $2-7 \mathrm{~mm}$ lang, spitz...................... trivialis.

5. Pflanze dichtrasig, selten mit Ausläufern; Deckspelzen nur rauh, ohne zottige

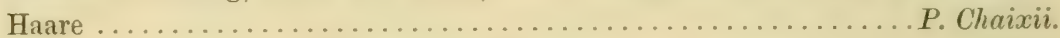

- Pflanze lockerrasig, ausläufertreibend; Dechspelzen unterwärts mit verbindenden Haaren . . . . . . . . . . . . . . . . . . . . . . . . . Pratensis.

6. Stengel an Grunde zwiebelartig rerdickt ............... bulbosa.

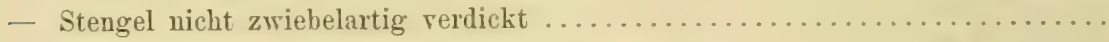

7. Stengel deutlich zweischneidig zusammengedrückt............ compressa.

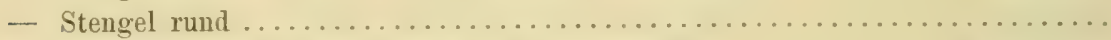

8. Blatthäutchen $2-3 \mathrm{~mm}$ lang, spitz bis stumplich............ palustris.

- Blatthäutchen sehr kurz, oft fast fehlend................ nemoralis.

115. (sp. 41). Poa annua L. Spec. plant. ed. 1. 68 (1753).

(. und -. Stengel zu mehreren, autrecht bis niederliegend. $0.05-0.3 \mathrm{~m}$ lang. glatt. Blattscheiden schwach zusammengedrückt, glatt. Blattfläche glatt. ofter am Rande sehr schwach rauh, $2-41-51 \mathrm{~mm}$ breit, mit kurz kappentörmiger spitze. Blatthäutchen bis 2 mm lang. stumpf bis stumpflich. Rispe locker. mit mehr oder weniger abstehenden, oft einseitig gestellten Ästen. (1-)3-5(-8) cm lang; untere Äste ohne oder mit einem grundständigen Zweige. Ährehen bis $8 \mathrm{~mm}$ lang. mit bis sieben Blüten. Hüllspelzen ungleich, untere efwa $1.5 \mathrm{~mm}$ lang. eimnerrig. obere etwa $2.5 \mathrm{~mm}$ ling. dreinervig. beide stumpflich. Deckspelzen bis reichlich $3 \mathrm{~mm} \mathrm{lang,}$ länglich. stumpt. undeutlich füntnerrig. am Grunde schwach behaart bis fast kahl. Blïte Januar bis Dezember.

All und anf IT egen. Wegrändern, Schutt und Gartenland, weniger auf Äckern. Wriesen und in Wäldern. vielfach an Ltern und in Gräben; durch das Gebiet gemein (auch auf allen Nordseeinseln).

Als erster Florist des Gebiets nemnt Weber die Art (Prim. Fl. Hols. 8 $[1780])$.

\section{Formen.}

1. Stengel aufrecht oder aufsteigend $\ldots \ldots \ldots \ldots \ldots \ldots \ldots \ldots .2$

- Stengel am Grunde oder der ganzen Länge nach liegend, nicht bewrurzelt oder an den Knoten wurzelnd.............. 4 
2. Stengel aufrecht oder aufsteigend, ziemlich fest; Rispenäste abstehend, nicht nickend................. 3

- Stengel anfstoigend, schlaff; Rispenäste verlängert. solulaff, an der Spitze oft nickend; Blätter verlängert, sehr schlaff:

f. umbrosa nor.f. - An sehr schattigen (oft feuchten) Orten nicht selten.

3. Rispe mit zahlreichen, drei- bis siebenblütigen Ährchen:

f. typica Beck Fl. Nieder-Oesterr. I. 84 (1890). - Häufigste Form.

Zerfaillt in zwei Farbenformen:

f. viridis Lej. et Court. Comp. Fl. Belg. I. 80 (1828). Ährchen grïn. - Hänfig.

f. picta Beck Fl. Nieder-Oesterr. I. 80 (1890). - Ährchen violett überlaufen. - Zerstrent.

- Rispe mit wenigen, (ein- bis) zwei- bis dreiblütigen Ährchen; Rispenäste sehr fein; Blattfläche etwa $1 \mathrm{~mm}$ breit; Stengel $5-10 \mathrm{~cm}$ hoch:

f. panciflora Fiek 69. Ber. Schles. Ges. II. 98 (1892). — So an trockenen, sandigen Orten zerstreut, stellenweise reichlich, z. B. Stormarn: Boberg!!. Hamburg: Hamm (J. A. Schmidt!). Oldenburg: Bliesdorf!!. Sylt: Klappholtal!!.

t. Stengel schlaff, liegend (oft im Wasser flutend), nicht wurzelnd:

f. aquatica Ascherson Fl. Brandenb. I. 844 (1864). - An Ufern und Gräben zerstreut, stellenweise nicht selten.

Bereits C. Timm erwälnt (Naturw. Ter. Hamb. N. F. IV. 76 [1880]) die Abart ohne Benennung rom Köhlbrand bei Hamburg. Anscheinend ist f. decumbens Nolte (in Hansen Herb. Schlesw.-Holst.-Lauenb. Fl. 1207 [18̄55]) (als Übergangsform?) hierherzuziehen!, ebenso vielleicht eine ron Knuth erwähnte Form (Fl. Schlesw.-Holst. 767 [1787]) : "Wurde von Pastor Jörgensen in Deezbüll (Tondern) mit langen Ausläufern beobachtet. " (Ausläufer?).

- Stengel verlängert, an den Knoten wurzelnd:

f. reptans Hauskn. Bot. Ver. 'Thür. IX. 7 (1891). - Bisher im Gebiete nicht beobachtet.

116. (adv. 67). Poa bulbosa L. Spec. plant. ed. 1. 70 (1753).

4. Stengel zu mehreren, am Grunde von zwiebelartig rerdickten Scheiden umhüllt, aufrecht, 0,05-0,3 m hoch, glatt. Blattscheiden etwas

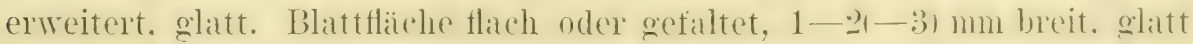
oder schwach rauh. Blatthäutchen - -3 mm lang, spitz uder stumpiliclı.

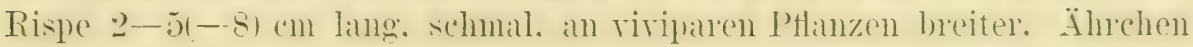


meistens 5-6 mm lang, vier- bis sechs- (bis acht-)blütig. Hüllspelzen dreinervig, wenig ungleich, untere $2,5 \mathrm{~mm}$, obere $3 \mathrm{~mm}$ lang, breiter. Deckspelzen 2(-2,5) mm lang, spitz, undeutlich fünfuervig; Nerven unterwärts zottig behart; Haare die Blüten verbindend. Blüte Mai und Juni.

Auf Schutt und Grasland selten verschleppt, zuweilen durch Jahre beständig.

H a m burg: bei der Dampfmühle Tandsbek (Zimpel 1896!) 1901, 1902!!, in Winterhude (Laban 1893!), im Eimsbütteler Holz (C. Timm 1859, Laban 1868!, Sparbjer noch 1890!), am Diebsteich in Altona (C. Timm 1885!, Laban 1887!). Itzehoe (Spalkhaver 1828!).

Alle übrigen Angaben der P. bulbosa sind unrichtig oder unsicher. Das gilt z. B. von Noltes Fundorten. Exemplare von Römnitz bei Ratzeburg (schon außerhalb des Gebiets in Mecklenburg-Strelitz) aus dem Jahre 1822! sowie solche von Neustadt, ebenfalls 1822 gefunden!, gehören zu P.pratensis. Auf ersteren Ort bezieht sich vielleicht die Aufählung dieser Art unter den Lauenburger Pflanzen durch Hornemann (Vid. Selsk. phys. Skrift. I. Deel. I. Haefte. 194 [1821]), wobei allerdings Voraussetzung wäre, daß Nolte, der schon 1820 bei Ratzeburg sammelte, $P$. bulbosa bereits vor 1822 gefundeu zu haben geglaubt hätte. Die erwähnte Hornemannsche Schrift enthält eine große Zahl unrichtiger, von Nolte veranlaßter Angaben. In dem Handexemplar seiner "Novitien" hat Nolte außer den beiden schon genamnten Orten noch aufgezählt ein angebliches Vorkommen in Mühlenberger Holz bei Blankenese (Hübener 1817), das Vorkommen bei Itzehoe sowie ein von Wolf angegebenes Auftreten bei Lübeck. Bei Lübeck fehlt indes P. bulbosa (vgl. Friedrich Fl. v. Lübeck 43 [1895]). Bei Mühlenberg suchten spätere Hamburger Floristen rergeblich (vgl. Sonder Fl. Hamb. 53 [1851]). Zwar gibt Hübener (der Sohn des von Nolte erwähnten Floristen) das Gras rom gleichen Orte wieder an (Fl. v. Hamb. 501 [1847]), doch beseitigt das die Zweifel nicht im mindesten. Die verschiedenen Nolteschen Vermerke wiederhnte Reichenbach (Vurles.-Verz. Hamb. 20 [18s1]) unter $P$. bulbifera "L.". Im Lübecker Herbar ist $P$. bulbosa in zwei Bogen von Hamburg vorhanden. Auf dem ersten heißt es: „Bei Reinbeck. Sonder."!, auf dem zweiten nur "Hamburg" !. Sonder erwähnt P. bulbosa weder in seiner Flora noch in der Festschrift zur Naturforscherversammlung in Hamburg 1876 von Reinbek, so daß mir die Aufnahme dieses Fundortes nicht ratsam erscheint. Bei Knuth steht (Fl. v. Schlesw.-Holst. 767 [1887]): „an der Elbe bei Altona“, als Finder J. J. Meyer, dessen Herbar aber P. bulbosa nicht von Altona enthält. Alle übrigen Bemerkungen (z. B. bei Borchmann, Knuth, Laban) gehen auf bereits erwähnte Orte zurück.

\section{Formen.}

f. m. vivipara Koeler Descript. gram. 189 (1802). - Ährchen in Laubsprosse auswachsend. - - So an allen Standorten, und zwar in Wrinterhude! und bei Itzehoe! ausschließlich.

Poa concinna Gaudin Agrost. Helv. I. 196 (1811) hat Zimpel angeblich bei der Wandsbeker Dampfmühle gefunden (Deutsche Bot. Monatsschr. XVI. 115 [1898]). Einen Zweifel äußern wenig später Ascherson und Graebner (Synopsis II. 1. 394 [1900]). Die Exemplare gehören zu P.pratensis $f$. collina!; ein Bogen wurde von Zimpel selbst als fragliche P.pratensis bezeichnet!. Von der Wollkämmerei am Reiherstieg stammende, als $P$. concinna benamnte Pflanzen sah ich nicht (vgl. Höck Bot. Centr.-Bl. XVII. Heft 1. 205 [1904]). 
117. (sp. 42). Poa nemoralis L. Spec. plant. ed. 1. 69 (1753).

4. Grundachse mit meistens kurzen, selten bis $20 \mathrm{~cm}$ verlängerten Ausläufern. Stengel $0,2-0,7(-1) \mathrm{m}$ hoch, aufrecht oder aufsteigend, glatt. Battscheiden eng. glatt oder schwach rauh. Blattflärhe 1-20-3) mum breit. zuweilen gefaltet. an Rande rauh. Batthäutehen sehr kum odey

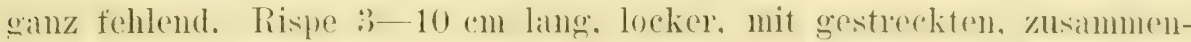

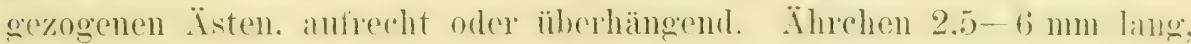
(ein- bis) zwei- bis fünf- (bis acht-bblïtig. Hüllspelzen wenig ungleiolı, 2-2.5) mm lang. dleinervig, am Kiele oberwäts rauh. spitz; Deckspelz('n bis 3 mm lang, undentlich fïnfnervig, am Mittelnerven und am Randnerven sowie am Rüblen weibharig gewimpert. stmmpflich bis spitz. IBlüte Juni und Juli, zuweilen später.

In Wäldern mud Gebüschen, an Abhängen und Wegen, zuweilen anch anf Wiesen und an Ltern häufig, doch im Westen seltenel; anf den Nordfriesischen Inseln nur auf Sylt: Keitum (Ostermeyer).

Die erste Erwälnnmg hat Weber (Prim. Fl. Hols. 8 [1780]).

\section{Formen.}

1. Ährchen größtenteils drei- bis fünf- (bis acht-)blütig, $2,5-6 \mathrm{~mm}$

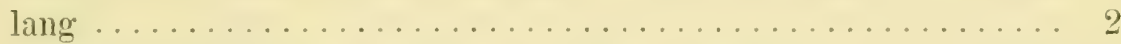

- Ährchen meistens ein- bis zwei- (bis drei-)blütig, 2,5-6 mm lang 5

2. Stengel glatt; Blattscheiden glatt oder schwach rauh........ 3

- Stengel und Blattscheiden rauh:

f. rigidula Mert. u. Koch Deutschl. Fl. I. 617 (1823). - Pflanze steif aufrecht; Blattfläche sehr schmal, gefaltet; Rispe aufrecht bis wenig überhängend, ziemlich dicht; Ährchen violett überlaufen. - H a m b u r g: „auf feuchten Waldwiesen und Triften" (Sonder Fl. Hamb. 53/54 [1851]). Bereits C. Timm vermutet in der Sonderschen Pflanze $f$. firmula (Naturw. Ver. Hanb. N. F. IV. 76 [1880]). Borchmann nennt $f$. vigidula ohne Standort (Fl. v. Holst. $76[1856]$ ).

3. Stengel aufrecht, starr . . . . . . . . . . . . . . . .

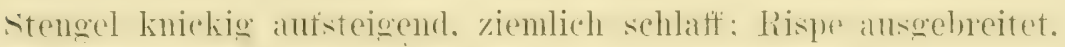
reichährig, ihre Äste mit bis über zehn Ilnrehen:

f. Reichenbachii A. u. Gr. Syn. Mitt.-Europ. Fl. II. 1. 409 (1900). - Auf fruchtbarem Boden nicht selten.

4. Rispe zusammengezogen; Blüten durch die Beharung der Deckspelzen verbunden :

f. coarctata Gaudin Agrost. Helv. I. 185 (1811). - An trockenen, sonnigen Orten zerstreut.

Dazu als Unterform:

f. rariflora A. u. Gr. a. a. O. II. 1. 409 (1900). - Pflanze bis $30 \mathrm{~cm}$ hoch, zart; Rispe mit drei bis acht thrchen. 
Lübeck: Kücknitz (Häcker!). Schleswig: Böcklund (Staacke!).

- Rispe mit alstehenden Ästen; Blüten nicht durch die Deckspelzenbehaarung verbunden:

f. firmela Gaudin a. a. O. I. 181 (1811). - Nicht selten.

5. Stengel derb; Rispe groß, ihre Äste mit zahlreichen Ährehen; Ährchen zwei- bis dreiblütig:

f. vulgaris Gaudin a. a. O. I. 179 (1811). - In Wäldern und

Gebüschen (doch auch an somnigen Orten) nicht selten.

- Stengel zart, schlaff; Rispe kleiner, mit zarten, nickenden oder hängenden Ästen; Äste wenigährig; Ährchen (ein- bis) zwei- bis dreiblütig:

f. tenelle Reichenbach Icon. I. t. LXXXVI. fig. 1639 (1834).-- An tiefschattigen (fenchten) Orten zerstrent.

Dazu als Unterform:

f. uniflora Mert. u. Koch a. a. O. I. 617 (1823). - Rispe traubig, mit drei bis sechs Ährchen; Ährchen einblütig. - Im Gebiete fraglich.

Ubergangsformen dieser Unterabart nach $f$. tenella treten hier und da auf, so z. B. Stormarn: Wellingsbüttel (Hb. Beyle!) in starker Annäherung an $f$. unittora; zu ihnen zählen f. subuniflora Reichenbach und f. micrantha Hornemann (Hb. Kopenhagen!).

118. (sp. 43). Poa palustris L. System. ed. 10. 874 (1759).

4. Grundachse verlängerte Ausläufer treibend. Stengel $(0,1-)$ $0,3-0,6(-1) \mathrm{m}$ hoch, aufrecht oder öfter aus knickigem Grunde aufsteigend, selten lis auf den oberen stengeltril liegend. Blattscheiden glatt, selten rauh. Blattfläche $2-3(-4) \mathrm{mm}$ breit, unterseits glatt oder schwach rauh, oberseits stärlier rauh. Blatthäutchen 2-3 mm lang, spitz oder stumpflich. Rispe $\frac{1}{4}-10(-30) \mathrm{cm}$ lang, schmal, mit rerlängerten, oft etwas schlaffen Ästen. Ährchen (3-)4-5(-6) mm lang, (zwei- bis) dreibis sechsblütig. Hüllspelzen schmal, spitz, dremervig. obere 2.5-3 mm. untere 2-2,5 mm lang. Deckspelzen bis 2,5 mim lang, sehr undentlich fünfnervig, stumpflich, ann Kiel und an Rande weit anfwarts schwach lin\% behaart. Blüte Juni bis August, vereinzelt bis Oktober.

Auf feuchten Wiesen und an Ufern, weniger in Gebüschen und

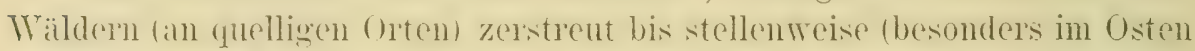
mol längs der Elher nieht selten, doch ant den Nordsecinseln bisher nur auf $\mathrm{Helgoland}$ : Sehweizerhansgarten (Hallicl) ijetzt rersehwunden). Zuerst von Buek (Hoppe Bot. Taschenb. 92 [1801]) erwähnt. 


\section{Formen.}

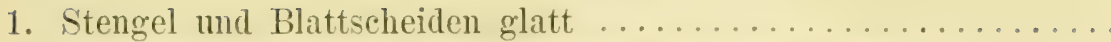

- Stengel und Blattscheiden schwach rückwärts rauh:

f. scabriuscula Ascherson Fl. Brandenb. I. 846 (1864). — Auf trockenem Boden; ob im Gebiet?

Die aus Schleswig von Prahl angegebene Pflanze (Krit. Fl. $25 \overline{5}$ [1890]) gehört zu P.trivialis!; Prahl selbst hat im Herbar die Bestimmung geïndert. Die Knuthschen Ausführungen sind nicht belegt und ganz unsicher; Häcker hat die Abart nicht bei Lübeck gefunden.

2. Ährchen (vier- bis) fünf- bis sechsblïtig, etwa $5 \mathrm{~mm} \operatorname{lang} . . . .33$

- Ährchen zwei- bis dreiblütig, (3-) $4 \mathrm{~mm}$ lang............ 4

3. Stengel hoch; Rispe groß, reichährig, ansgebreitet locker:

f. glabra Ascherson a. a. O. I. 846 (1864). - Verbreitetste Form.

Dazu als Unterform:

f. radicans nov. f. - Stengel am Grunde niederliegend, hier an den Knoten wurzelnd und ans ihnen Äste treibend. - Hamburg: Harvestehude (Zimpel 1890!). Angeln: Ausacker (Hansen!).

- Stengel niedrig, 0.1-0.3 m hoch; Rispe kleiner, zusammengezogen:

f. muralis Ascherson a. a. O. I. 846 (1864). - An trockenen

Orten des Elbvorlandes und in den Ritzen der Steindeiche von Lauenburg bis Hamburg (schon Sonder!)!!

4. Pflanze kräftig; Rispe reichährig, bis $0,3 \mathrm{~m}$ lang:

f. fertitis Reichenbach Icon. I. t. LXXXVII. fig. 1647 (1834). H a mburg: auf den Elbinseln (Sonder).

- Pflanze schwach; Rispe bis $15 \mathrm{~cm}$ lang, mit dünnen, schlaffen Ästen; Äste mit höchstens acht Ährchen:

f. effusa Reichenbach a. a. O. fig. $16 \pm 6$ (1834). - An schattigen, feuchten Orten zerstreut, von Hansen 1847! in Lauenburg zuerst gesammelt.

Dazu als Unterform:

f. depauperata A. u. Gr. Syn. Mitt.-Europ. Fl. II. 1. 418 (1900). - Stengel bis $0,3 \mathrm{~m}$ hoch; Rispe kurz, mit drei bis acht Ährchen. - Hamburg: Geesthacht (J.A.Schmidt!), Kl.Grasbrook (Zimpel!), Fuhlsbüttel!!

\section{Mißbildungen.}

f. m. vivipara Graebner Naturf. Ges. Danzig N. F. LX. 1. 343 (1895). Ährehen in Laubsprosse auswachsend. - Stormarn : bei der Alten Mühle bei Bergstedt (Röper 1907!). 


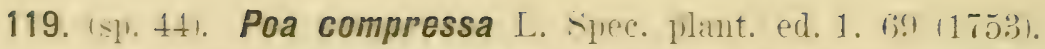

4. (irundarhse nit l,is $0.3 \mathrm{~m}$ langen Ausläufern. stenger anfrecht orlor anfsteigend. $0.2-0.5-11 \mathrm{~m}$ hoch. zweischneidig zusammengedrückt.

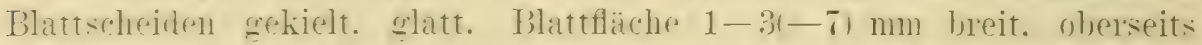
ranh. zuweilen geraltet. Blatthäutchen rotwa $11-2)$ mm lang. stmmpt

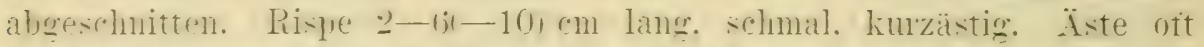

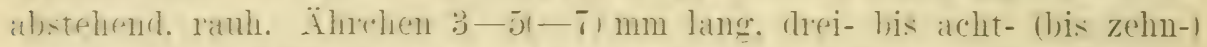
b]ütig. Hällsuelzen dentlich oder undentlich dreinervig. spitz. untere $2(-2,5) \mathrm{mm}$, obere $2,5(-3) \mathrm{mm}$ lang. Deckspelzen $2(-2,5) \mathrm{mm}$ lang,

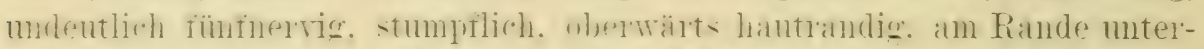
wärts mit rexlindenden Haaren. Blüte Imi und Juli, rifter bis oktober.

An Wegrändern, an Abhängen und auf Wällen, seltener auf Grasland. zuweilen auf schutt: im sibllichen (iel)iet nicht selten. nordwärts zerstreut bis Hadersleben: Sommerstedt. im Westen nur bei Itzehoe:

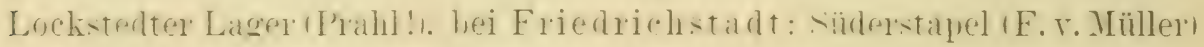
und auf Helgoland: am Falm (Ascherson).

Die erste Nennung der Art hat Weber (Prim. Fl. Hols. 8 [1780]). Frühere Helgoländer Angaben (Hallier usw.) sind unsicher.

\section{Formen.}

Zerfällt bei uns in zwei Rassen:

1. Stengel $0,2-0,5$ m hoch; Rispe kurz; ت̈hrchen drei- bis sechs- (bis sieben-)blütig; Deckspelze am Rande unterwärts zottig:

var. typica A. u. Gr. Syn. Mitt.-Europ. FI. II. 1. 420 (1900). Häufigste Form.

Dazu als Unterformen:

f. muratis A. u. Gr. a. a. O. II. 1. 420 (1900). - Stengel niedrig, oft starr, mit bis fünf Stengelblättern; Rispe klein, stark zusammengezogen. - An trockenen Orten bis zur Apenrader Föhrde!! sehr zerstreut, schon von Sonder (Fl. Hamb. 53 [1851]) erwälnt. - Die Blattausbildung dieser Form erinnert offer an $f$. polynoda A. II. Gr.

f. umbrosa Beck Fl. Nieder-Oesterr. I. 82 (1890). - Rispe mit verlängerten, schlaffen, abstehend-ïberhängenden Ästen.

- In Übergängen zerstrent, typisch z. B. Oldenburg: Heringsdorf (J. Schmidt!).

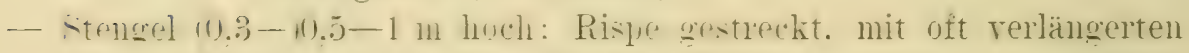

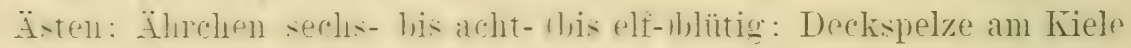
und am Rande rauh (zuweilen schwach zottig):

var. Langiana Koch Syn. Fl. Germ. et Hels. ed. 2. 932 (1844). -Bisher nur Pinneberg: am Flottbecker Elbufer (Sonder, Fl. 
Hamb. p. 56, Hb. Kiel!), am Elbufer bei Schulau 1911!!. Bei Hamburg auf Schutt: Georgswärder (Kausch!), St. Pauli (C. 'Timm), am Eppendorfer Moor (Laban 1886!), Winterhude (Zimpel 1891!).

Dazil als Unterform:

f. paradoxa nov. f. - Stengel völlig rund. - Pinneberg: Schulauer Elbufer!!. - Es kam sich um keine Hybride und um keine andere Art handeln, da die Pflanze völlig mit der var. Langiana in den Merkmalen übereinstimmt, nur daß der Stengel rund ist.

\section{Mißbildungen.}

f. m. ramifera nov. f. - Pflanze aus dem obersten Knoten mit einem $35 \mathrm{~cm}$ langen. zusimmengedrückten, blattlosen, rispentragenden Aste. Pinneberg: Schulau!! (bei var. Langiana).

f. m. bracteata A. Christiansen nov. f. - Rispe am Grunde mit laubblattartigem Tragblatt. - Eckernförde: Grasholz (A. Christiansen!).

120. (qsp). 9). Poa Chaixii Villars Fl. Delph. in Gilibert Syst. Plant. Eur. I. 7 (1785).

4. Grundachse dichtrasig, selten mit Ausläufern. Stengel $0,5-1,2 \mathrm{~m}$ hoch, anfrecht oder aufsteigend, glatt. Blattscheiden deutlich rauh. gekielt. Blattfläche $(3-15-10(-15) \mathrm{mm}$ breit, unterseits glatt, oberseits schwach. am Rande stark rauh. Blatthüutchen breit, stumpf, bis $1 \mathrm{~mm}$ lang. Rispe bis $20(-25) \mathrm{cm}$ lang, locker mit abstehenden, überhängenden Ästen, seltener zusammengezogen. Ährchen (t-16-9 mm lang. (drei- bis) rier- bis fünflblütig. Hüllspelzen schmal, spitz, untere einnervig. (2-)3 mm lang, obere dreinervig, (2,5-) $4 \mathrm{~mm}$ lang, am Kiele rauh. Deckspelzen bis 4 mm ling. mit fünf ziemlich deutlichen Nerven, kahl; Nerven rauh. Blïte Juni und Juli.

In Wäldern und Parks sehr zerstrent im östlichen, selten im westlichen Gebiet, nicht auf den Nordseeinseln.

L a ueuburg: Ratzeburg (Nolte 1820)!. Sonder). Friedrichsruh (Sickmann) am Wege nach Kasseburg (Laban 1885!). stormarn: Hahnheide bei Trittan (Hübener, sonder), Grönwohld (J. A. Schmidt 1878!). Pinneberg: Flottbek (C. Timm, W. Hansen 1887!) Lübeck: Teufelssmmpt bei Timmendorf (Prahl 1892!). Kiel: Barsbek (Nolte 1828!) und schoinberg (Nolte 1830 !, .J. J. Meyer!), beim Knnooper Fährhans 1A. Christiansen 1909!). Hadersleben: Christianstal (Prahl 1872!). Tondern: Schackenburg bei MIögeltondern (Prahl 1885!). 
Nolte veröffentlichte seinen Nachweis der Art 1826 (Nov. Fl. Hols. 13. Sif hommt zuweilen mit Auslänfern ror: so fand sie T'ralle. und zwar walnerheinlich im Teufelssumpe bei Timmendenf: E. H. L. Krause nemnt als Fundort dieser Fonn Eutin (Flonistische Notizen II. 5). Eine ähnliche Form sah vielleicht Kunth wogl. Fl. r. schlesw.-Holst. 769 [1887]).

\section{Formen.}

Vom Typus weichen ab:

f. remota Fries Nov. Fl. Suec. ed. 2. 11 (1828). - Pflanze mit sehr weit locker ausgebreiteten, schlaffen Rispenästen. - Pinneberg: Flottbek (C. Timm, Jaap 1890!). Kiel: Barsbek und Schönberg (Nolte!). Tondern: Mögreltondern (Friederichsen 1898 !, bestimmt als $f$. laxior .

f. ancustifulin nov. 1. - Blattliachen auffallig schmal. nur 3-5 mm breit. - Tondern: Schackenburger Park (Prahl!).

f. mbens A. u. Gr. Syn. Mitt.-Europ. Fl. II. 1. 423 (1900). Ahrchen violettbraun ïberlaufen. - Kiel: Knooper Fährhaus (A. Christiansen!).

121. (sp. 45). Poa trivialis L. Spec. plant. ed. 1. 67 (17503).

2. Grundachse locher, oft ausläufertreibend. Stengel $(0,3-10,5-0.8$ $1-1.251 \mathrm{~m}$ lısch. aufrecht oder aufsteigend, meistens rückwärts rauh, rund. Blattscheiden grekielt. rürkwärts rauh oder selten glatt. Blattfläche 2-4t-it mm hreit. selten breiter. heidersits. besonders oberseits, rauh. Blatthäutchen spitz. verlängert. lis 7 mm lang. Pispe $5-12-201$ cm lang. auscelswitet oder zusammengezogen. Ährchen 12-13-5 mm lang. (ein- bis zwei- bis füntblütig. Hüllspelzen schmallanzettlich. spitz. ungleich. untere (1,5-12 mm lang. eimnervig, obere (2-) 2.5-3 mm lang. dreinervig. Dechepelzen 2.5-3.5) mm lang. mit fünf starken Nerren, an den Nerven behaart, sonst fast kahl, am Grunde zottig. Blüte Mai bis Juli, zuweilen später.

Auf Wiesen und Triften, in Wäldern und Gebüschen durch das Gebiet meist häufig. auch auf Fijhr. Amrum. Srlt. Riom und Helgoland zerstreut.

Zuerst genannt ron Weber (Prim. Fl. Hols. 7 [1780]).

1. Formen.

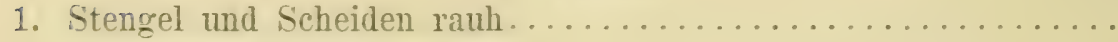

- Stengel und Scheiden glatt:

f. glabra Döll Rhein. Fl. 92 (1843). - Zerstreut. La u enburg (Nolte, Hb. Kopenhag.!): Besenhorster Elbvorland!!. S tor- 
man'n: Oejendorf (J. A. Schmidt 1869!), Alt-Rahlstedt!!. Hamburg: Zollenspieker!!, Horn 1874 und Hamm beim Hasselbrook 1869 (J. A. Schmidt!). Sylt: Keitum!!. Helgoland (Brody!). - Mit der Form finden sich Ubergïnge.

2. Rispe mit aubecht abstehenden bis fast anfechten Isten, ziemlich dicht:

f. vulgaris Reichenb. Icon. I. t. LXXXIX fig. 1653 (1834). Verbreitetste Form.

Dazu gehören :

f. stricta Döll Fl. v. Baden 180 (1857). — Blattscheiden und Ährchen braun bis violett ïberlaufen. - An trockeneren, sonnigen Orten zerstreut.

f. pallescens Stebl. u. Volk. Schweizer Gräsersamml. n. 226 (1895). - Ahrchen gelblich. Stengel dünn, etwas schlaff. - Lauenburg: Moor östlich von Anker!!.

f. arida nov. f. - Untere Blätter eingerollt. - Lauenburg: auf trockenem Sandboden des Besenhorster Elbvorlandes 1911!!.

- Rispe mit abstehenden, zuweilen überhängenden Ästen, weit ausgebreitet:

f. effusa A. u. Gr. Syn. Mitt.-Europ. Fl. II. 1. 426 (1900).An schattigen Orten, seltener. - Nach Hackel (Allg. Bot. Zeitschr. XIII. 11 [1907]) sind f. vulgaris und $f$. effusa zu vereinigen.

\section{Mißbildungen.}

f. m. ramifera nov. f. - Einem oberen Knoten entspringt ein rispentragender Seitenast. - Kiel: Gr. Westensee (A. Christiansen!).

122. (s). 46). Poa pratensis L. Spec. plant. ed. 1. 67 (1753).

4. Grundachse locker, ausläufertreibend. Stengel $(0,1-) 0.2-1 \mathrm{~m}$ hoch. aufrecht oder aufsteigend. glatt. rund oder schwach zusammenEedrückt. Blattscheiden nicht gekielt, glatt. Blattläche $1-3(-5)$ mm hreit. flach oder eingerollt. unterseits slatt, oberseits und an Rande schwach jauh. Blatthäutchen bis $1 \mathrm{~mm}$ lang, breit abgeschnitten. Risue kurz, zusammengezogen oder lang, ansgebreitet, mit zuweilen schr verlängerten Asten. Ährehen 12- $14-6 \mathrm{~mm}$ lang. meisten zwei- bis fümfblütig. Hüllspelzen ungleich, spitz. untere eimervig, 2- 2, é mm lang. obere dreinervig. 2.5-3.5 mm lang. I)eckspelzen spitzlich, stak fünfnervig. lis $3,5 \mathrm{~mm}$ lang; an den Nerven rauh, am Grunde zottig. Blüte Mai bis Juli, einzeln spåter. 
Aut IViesen und Triften. anf Heide- und Dïnenbolen. an Tregrändeln und Fesdrainen. in Gebüschen und Wäldern häufig: anch auf Föln. Amrum. Sylt, Röm, Norderoog und Helgoland.

Die erste Ermähnung findet das Gras bei Teber (Prim. Fl. Hols. 8 [1780]), und zTrar unter Nr. 73 (der Trpus) und Nr. 74 (f. angustifolia als Art).

\section{Formen.}

1. Alle Blätter flach......................... 2

- Alle Blätter oder nur die Grundblätter eingerollt ........... 6

2. Blätter schmal, gegen die Spitze allmählich verschmälert ..... 3

- Blätter 3-j mm breit, an der Spitze plötzlicher rerschmälert, happenförmig zusammengezogen.................

3. Stengel rund:

f. vulgaris Gaudin Agrost. Helr. I. 212 (1811). - Häufigste Form, in der Regel $0,4-0,8 \mathrm{~m}$ hoch.

Dazu als Unterformen:

f. glauca Lej. et Court. Comp. Fl. Belg. I. 82 (1828). Pflanze graugrün, auffällig lockerwiichsig. - H a m bux g: Winterhude (Kausch 1879!). Stormaln: zrischen Schönau und Tritzhave!!. Lïbeck: Blankensee!!. Oldenburg: Grube Nolte!). Kiel: Streitberg und Kühren (A. Christiansen!).

f. arenaria nor. f. - Pflanze lebhaft grïn; Stengel 0,1 bis $0,2 \mathrm{~m}$ hoch, oberwärts blattlos; Blattfläche des obersten Blattes sehr kurz, drei- bis riermal so kurz wie die Scheide; Rispe kurz, fast eiförmig; Rispenäste oft mit einem oder keinem grundständigen Zweige. - Terwandt mit $f$. heterophylla (Scheele) A. u. Gr., aber ohne kappenfürmig zusammengezogene Blattspitzen. Auf Sand- und Moorboden z. B. Lauenburg: Besenthaler Wiesen (J. A. Schmidt!). Hamburg: Bergedorf 1864. Tinterhude 1873 (J. A. Schmidt!). Stormarn: zwischen Schiffbek und Tandsbek (J. A. Schmidt!). Lübeck: Beidendorf (J. Schmidt!). Kiel: Mönkeberg und Brammerteich (A. Christiansen!). Tondern: Deezbiill (Jörg’ensen!).

- Stengel zusammengedrüickt:

f. planiantmis (TTeber Prim. Fl. Hols. Suppl. 3 [1787]). Zerstreut.

TTeber unterschied die Abweichung als Art. Als solche weist schon Hornemann sie zurück (Vid. Selsk. phrs. Skr. I. Deel I. 186 [1821]). Die ältesten Herbarexemplare der Form sammelte Bargum 1797 bei Kiel!. Hierher zählt $P$. compressa J. J. Meyer Herb. zum Teile!. 
4. Rispenäste mit zwei oder mehr grundständigen /wweigen ..... i)

- Rispenäiste mit keinen oder einem grundständigen Kweige:

var. costate Hartman Handb. Scand. Fl. 2. Uppl. (1832)

Pflanze blaugrün, meistens niedrig; oberste Blattfläche kurz, starr; Rispe kurz; Ahrehen meistens dreibliitig.

Holstein (Nolte, Hb. Kopenhagen!). Stormarn:

Brenner Moor bei Oldesloe!! (hochwïchsige Form). Kiel:

Möltenort (Erichsen 1886!), Dietrichsdorf, Strande, Stein,

Barsbecker Deich und Eckernförde: Ohrt, Schwansener

See (A. Christiansen!). Amrum (Jessen nach V. Seemen).

Fölır: Gothing!!

5. Pflanze lebhaft grün; Stengel hoch; Ährehen vier- bis fümfblütig:

f. latifolia Mert. u. Koch Deutschl. Fl. I. 612 (1823). - Au fruchtbaren Orten nicht selten.

Pflanze blaugrün, niedrig (etwa 0,1 m hoch); Ährehen drei- bis fiinflulïtio:

f. subcoenulea A. u. Gr. Syn. Mitt.-Europ. Fl. II. 1. 433 (1900). - So seltener, z. B. Kiel: am Drecksee, Brammerteich, Gaarden, Eckernförde und Rendsburg: Ostenfeld (A. Christiansen!).

6. Grundbläter gefaltet oder eingerollt, Stengelblätter flach ......

Alle Blätter gefaltet oder eingerollt:

f. setacer Döll Rhein. Fl. 91 (1843). - Auf trockenem Boden zerstrent. Hamburg: Geesthacht (Laban 1891!), Besenhorst und Warwisch!!. Kiel: Mönkeberg (A. Christiansen!). Angeln: Langballigau (Hansen!, nicht ganz typisch).

Die Pflanze Hansens (im Hb. J. A. Schmidt) war als $f$. strigosa bezeichnet; unter demselben Namen wurde die Form von Hansen ausgegeben (Hb. Schlesw.Holst.-Lauenb. Fl. 1206 [1855]). P. strigosa ist aber eine südliche Rasse. Knuth führt sie (Fl. Nordfries. Ins. 147 [1895]) olne genaueren Fundort auf.

7. Pflanze niedrig, 0,1-0,2 m hoch; Blattfläche ziemlich kurz und derb:

f. collina Schur Nat. Ver. Siebenb. IV. 88 (185̃3). - Ant́ sandigem Boden zerstreut.

- Pflanze kräftig; 0,3-0,6(-0,8) m hoch; Blattfliche oft schlaff:

f. angrestifotia Smith Fl. Brit. 105 (1800). - Nicht selten; anch auf den Nordfriesischen Inseln beobachtet.

Dazu als Unterformen:

f. hivtula A. u. Gr. a. a. O. II. 1. 432 (1900). - Blattscheiden (und Blatttlächen) wenigstens teilmeise dicht kurzhaarig. - Apentrade: Strandhöhen bei der Schleifmuihle!!. 
f. straminea Rother in Ascherson Fl. Brandenb. I. 848 (1864). - Ährchen gelblichweiß. - Hamburg: Curslack $1904 ! !$

f. laxa P. Junge Jahrb. Hamb. Wiss. Anst. XXII. 65 (1905). — Blattfläche lang, sehr schlaff; Rispenäste verlängert, schlaff, ïberhängend. - Hamburg: in feuchten Gebüschen der Curslacker Marsch 1904!!.

123. (adv. 68\%. Poa persica Trinins in C. A. Meyer Terz. Kankasus 18 (1831).

$\odot$. Stengel zu mehreren, aufsteigend, $0,1-0,4 \mathrm{~m}$ hoch, glatt. Blattscheiden glatt. Blattfläche 2-4 nm breit, unterseits glatt, oberseits rauh. Blatthäutchen spitz, bis 9 mm lang. Rispe meistens $5-10 \mathrm{~cm}$ lang, ausgebreitet, ihre unteren $\ddot{A}$ ste mit sechs oder mehr grundständigen Zweigen. Ährchen 6-7 mm lang, meistens vier- bis sechsblütig. Hüllspelzen ungleich, spitz, untere etwa $1.5 \mathrm{~mm}$ lang. einnervig, obere etwa $2 \mathrm{~mm}$ lang. dreinervig. Deckspelze bis $2,5 \mathrm{~mm}$ lang, oberwärts hautrandig, stumpf, mit fünf undeutlichen Nerven. Blüte Juni.

Auf Schutt selten.

Hamburg: Wollkämmerei am Reiherstieg (.J. Schmidt 1896 !, Deutsche Bot. Monatsschr. XTI. 115 [1898]). Kiel: Teumühlen (A. Christiansen 1909 !).

\section{Gattung:}

\section{Briza.}

L. Gen. plant. ed. 5. 32 (1754) z. T'.

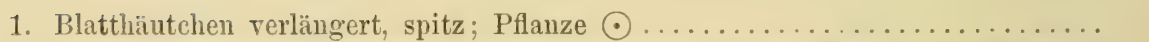

- Blatthäutchen bis $1 \mathrm{~mm}$ lang, breit abgeschnitten; Pflanze 24 ..... B. media.

2. Ährchen über $7 \mathrm{~mm}$ lang; Deckspelzen bis $6 \mathrm{~mm}$ lang; Rispe armährig.

B. maxima.

- Ährchen 3-4 mm lang; Deckspelzen bis $3 \mathrm{~mm}$ lang; Rispe reichährig.

$$
\text { B. minor. }
$$

124. (adt. 691). Briza maxima L. Spec. plant. ed. 1. 70 (1753).

$\odot$. Stengel zu mehreren, aufrecht oder aufsteigend, $0,2-0,5 \mathrm{~m}$ hoch, chatt. Blattscheiden glatt, obere schwach erweitert. Blattfläche 2-5 mm breit, unterseits glatt, oberseits und an Rancle schwach rauh. Blatthäutchen spitzlich, bis $5 \mathrm{~mm}$ lang. Rispe. $3-5(-10) \mathrm{cm}$ lang, mit (zwei bis) drei bis acht Ährchen. Ärchen 7-12(-20) mm lang, meistens fïnf- bis zehnblütig. Hülsspelzen breit eiförmig, stumpflich, $14,5-15-6 \mathrm{~mm}$ lang, grimlich mit gelbbräunlichem Rande, später bram. Deckspelzen bis 


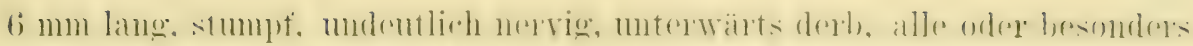

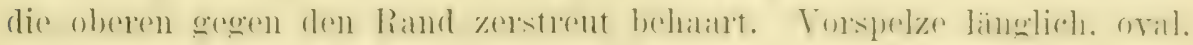
$3 \mathrm{~mm}$ lang. Blüte Mai bis Juni.

Auf Gartenland (und Schutt?) selten verwildert.

Stormarn: Trellingsbüttel (Nolte 1821!).

Eine Angabe der Art für Hamburg: Winterhuder Bruch (Laban in A. Junge Ver. Naturw. Unterh. Hamb. VII. 98 [1890]) dürfte sich auf B. minoi beziehen.

125. (sp. 47). Briza media L. Spec. plant. ed. 1. 70 (1753).

4. Grundachse lockerrasig. Stengel meistens aufrecht, $\left(0,05^{-}-\right)$

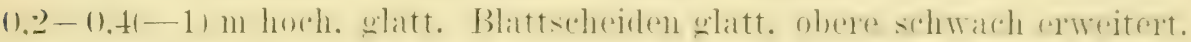
Blattfläche $2-3(-4) \mathrm{mm}$ breit, unterseits glatt, oberseits schwach, am Ramde starker ratuh. Blatthäutrhen his 1 mm lange. breit abgeschnitten. Rispe 3-10(-15) cm lang, breit, mit zahlreichen Ährchen. Ährehen $(2-) 4-\check{\partial}(-7) \mathrm{mm}$ lang, (zwei- bis) vier- bis sieben- (bis zehn-)blütig. Hüllsprelzen (2)-1:3-3.5) mm lang. stmmptlich. Weishantrandig. Derkspelzen

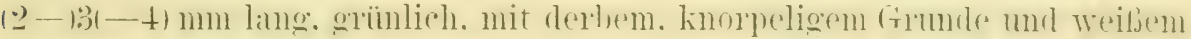

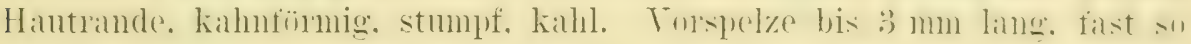
lang wie die Dechspelze. Blüte Mai bis Juli.

Auf Wiesen und Triften, Weniger auf IIooren, in Täldern und

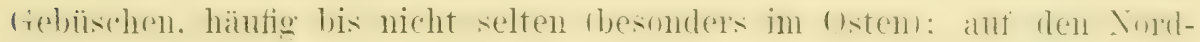
friesischen Inseln nur anf Sylt und Föhr (Schiötz).

Weber nennt als erster $B$. medin aus dem Gebiet (Prim. Fl. Hols. 8 $[1780])$.

\section{Formen.}

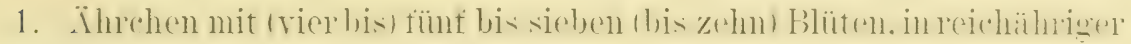
Rispe; Pflanze über $0,2 \mathrm{~m}$ hoch ................. 2

- Ährchen mit zwei bis drei (bis vier) Blüten, klein, in armähriger Rispe:

f. panciflora A. u. Gr. Syn. Mitt.-Europ. Fl. II. 1. 141 (1900). Pflanze nur 5 - $20 \mathrm{~cm}$ hoch. - Lauenburg: auf Moorboden im Delvenautal bei Göttin!!.

Die Pflanze wurde im Jahrb. Hamb. Wriss. Anst. XXII. 65̄ (1905̄) als $f$. pumila abgetremnt, unterscheidet sich aber anscheinend trotz des fast fehlenden Blatthäutchens nicht wesentlich ron $f$. parciflora.

2. Spelzen mehr oder weniger violett überlaufen ............

-.. Spelzen blaßgiün :

f. albida Lejeme Rev. Fl. Spa 17 (1824). - La u euburg: im Delrenantal bei Göttin (J. Schmidt)!! - Einen Ubergang fand Bock 1891! in Angeln: Klein-Solt. 
3. Pflanze 0,7-1 m hoch, mit großer, reichähriger Rispe; Ährchen sechs- bis neunblütig:

f. major Petermann Analyt. Pfl.-schlüss. 545 (1846). - Zerstreut; z. B. Lauenburg: beim Rothenhause (Nolte1821!), Techin!! Stormarn: Willinghusen (J.Schmidt!), Pinneberg: Wulfsmühle!!. Plön: Damsdorf!!. Kiel: mehrfach (A. Christiansen!).

- Pflanze 0.2-0.5 m hoch. mit kleinerer Rispe: Ährchen meistens vierbis sechsblütig:

f. typica A. u. Gr. a. a. O. II. 1. 441 (1900), - Häufigste Form. Dazı als Unterform:

f. multiflora nov. f. - Ährchen mit acht bis zehn Blüten. - Lauenburg:Friedrichsruh(J.A.Schmidt!). Übergangsformen zerstreut.

126. (adv. 70). Briza minor L. Spec, plant. ed. 1. 70 (1753).

$\odot$. Stengel einzeln oder zu mehreren, am Grunde öfter verzweigt. Stengel meistens $0.1-0.3 \mathrm{~m}$ hoch, aufrecht oder aufsteigend, unter den oberen Knoten rïckwärts rauh. Blattscheiden glatt. Blattfläche in der Regel 2-5 mm breit, unterseits glatt. oherseits rauh. Blatthäutchen schmal, spitzlich, 4-65-10) mm lang. Rispe reichährig. Ährchen $3-4 \mathrm{~mm}$ lang, drei- bis siebenblütig. Hüllspelzen 2-2.5 mm lang. eiförmig. stumpf, mit weifem Hantrande. Deckspelzen breit-eiförmig. stumpf. an Grunde (lerb. glänzend, weifhäutig berandet. Torspelze 1.5 mm lang. Bliite Juni bis Juli.

Auf Schutt bei Hamburg selten.

Hamburg: Winterhuder Bruch (.T. Schmidt 1890!) (Deutsche Bot. Monatsschr. X. 125 [1892]).

46. Gattung.

\section{Catabrosa.}

Pal. Beauv. Agrost. 97 (1812).

127. (s1). 48). Catabrosa aquatica Pa]. Beaur. Agrost. 97 (1812).

7. Grundachse lockerrasig. Init oft stark rerlängerten Ausläufern. Stengel selten aufrecht, in der Regel am Grunde niederliegend. oberwärts aufsteigend, $0,1-0,5 \mathrm{~m}$ lang. glitt. Blattscheiden glatt. Blattfläche 2-7 mm breit, röllig glatt. Blatthäutchen 1-2(-4) mm lang, stumpflich. 
Rispe 4-12(-20) cm lang, ihre Äste vor der Blüte aufrecht, nach der

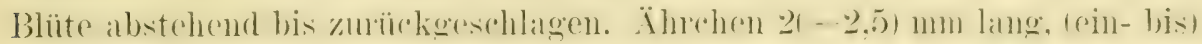

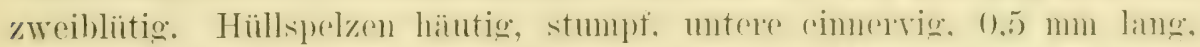

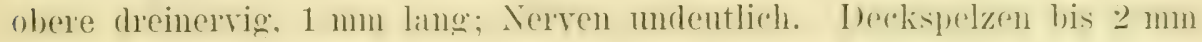
lang, mit drei starken Nerven, stumpflich. Blïte Mai bis Oktober.

An Quellen, Bächen, Gräben, an Fluß- und Seenfern durch das (iebiet \%erstrent: anf den Vordfriesischen Inseln mur anf Föhr: Boldixmm

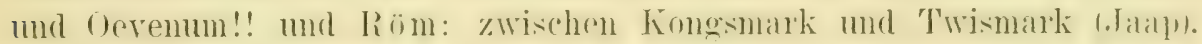

Die erste Erwähnung gibt für Holstein Weber (Prim. Fl. Hols. 7

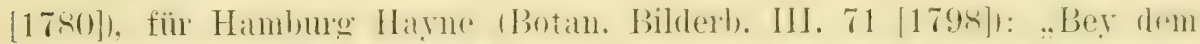
Eppendorfer und Winterhuder Mon ". Ton ersterer stelle liegen Exemplare vor (J. J. Мeyer 1816!).

Vom Typus weichen ab:

\section{Formen.}

f. subumiflor Gray Nat. Arrang. 133 (1821). - Ährchen größtenteils einblütig, in oft kleiner Rispe; Stengel oft dünn, niedrig; Blätter oft schmal und gefaltet. - Zerstrent. Lauenburg: Büchen (Brick!). Stormarn: Bredenbeker Teich!!. Hamburg: Farmsener MLor (Jaap 1891!), Hellbrook (C. Timm!). Kiel: Flintbeker MLor (A. Christiansen!). Rendsburg: bei der Seemühle (Lange als var. subtilis Hooker). Husum: Oster-Ohrstedt (A. Christiansen!).

f. stricte C. H. Schultz in Hegi Fl. v. Mitt.-Europ. I. 288 (1906). - Stengel aufrecht, 5-15 cm hoch. - Lauenburg: auf feuchtem Sandboden bei Escheburg!!.

f. purpurea A. Christiansen nov. f. - Ganze Pflanze rot überlaufen. - Eckernförde: am Schilksee (A. Christiansen!).

\section{Gattung.}

\section{Glyceria.}

Rob. Brown Prodlr. I. 179 (1810).

1. Deckspelzennerren gleich stark ........................... 2

- Deckspelzennerven abwechselnd länger, stärker und kürzer, schwächer...... ذ

2. Deckspelzen $5-6 \mathrm{~mm}$ lang, spitz...................... fluitans.

- Deckspelzen $3, \tilde{\partial}-4,5 \mathrm{~mm}$ lang, stumpf.................... plicata.

3. Stengel derb, aufrecht; Rispe sehr reichährig; Ährchen seitlich zusammen-

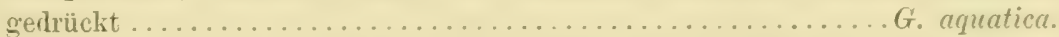

- Stengel zarter, aufsteigend; Rispe nicht reichährig; Ährchen rundlich. G. nemoralis.

128. (s). 49). Glyceria fluitans R. Brown Prodr. I. 179 (1810).

4. Grundachse lockerrasig, mit Ausläufern. Stengel meistens aufsteigend, 0.4-0,61-1, m liang; glatt. Blattscheiden glatt. Blattfliche 
besonders am Mittelnerv (unterseits) und am Rande stark rauh, $3-7(-12) \mathrm{mm}$ breit. Blatthäutchen bis $10 \mathrm{~mm}$ lang. Rispe $10-30 \mathrm{~cm}$ lang. mit zusammengezorenen. nu in der Blüte abstehenden Ästen, einseitig gestellt. Ährehen 11-26 mm lang. (ripr-bis fünf- bis zwölfblütig. Hüllspelzen luäutg. etwas glänzend. eimnervig. stumpf. untere bis 3,5. obere bis $4.5 \mathrm{~mm}$ lang. Deckspelzen spitzlich. 5-5.5-6) mm lang, mit deutlichen Verren. Torsuelze so lang wie die Deckspelze. spitz. Antheren etwa $3 \mathrm{~mm}$ lang. violett. Blüte Juni bis Juli, nicht selten bis oktober.

An Ufern, in Gräben, in Sümpfen und auf feuchten Wiesen häufig, anch anf den Vordtriesischen Inseln. Helgoland: Salskuhle früher (Hoffmamnı. Föhr (S.'hiötz) verlsreitet!!. Amrum: Steenodde (Ramkiaer. Togelkoje!!. Sylt IRamukiaer, Taap) nicht selteri!: und Röm: rerlmeitet in der Kulturzone (Jaap)!!.

Weber zählt $G$. fuitans als Festuca fuitans auf (Prim. Fl. Hols. 8 [17s(1)]. Für Dïnemark wird die Pflanze unter diesem Xamen sehon ron reder reszeirhnet (Flona I)anisa t. 237 [1765]): die Ablildmg zeigt indes kurze Spelzen und kurze, gelbe Antheren, so daß sie vielleicht zu G. plicata zul ziehen ist.

\section{Formen.}

Vom Typus weichen ab:

f. loliace Ascherson Fl. Brandenb. I. 850 (1864). - Rispe fast (oder völlig) traubig. - So nicht selten, besonders an trockenen (austrocknenden) Örtlichkeiten.

Dazu als Unterform:

f. pumila Vimm. u. Grab. Fl. Siles. I. 71 (1827). - Rispe mit höchstens sechs Ährchen; Stengel nur bis $25 \mathrm{~cm}$ hoch. - So mit der Abart zerstreut, meistens spärlich. f. latifotia Beck Fl. Nieder-Oesterr. I. 92 (1890). - Blätter 10-12 mm breit. - Auf nährstoffreichem Boden, selten. La uenburg: Friedrichsruh!!. Kiel: Gaarden (A. Christiansen!).

\section{9. (1). 5). Glyceria plicata Fries Nor. Mant. III. 176 (1842).}

4. Grundachse lockerrasig, ausläufertreibend. Stengel meistens

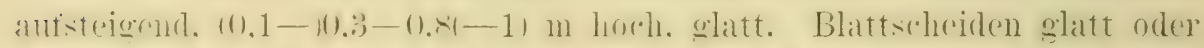

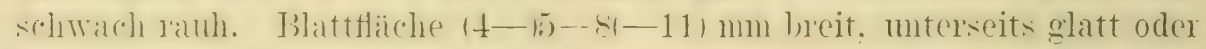

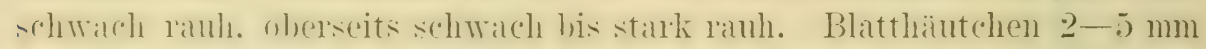

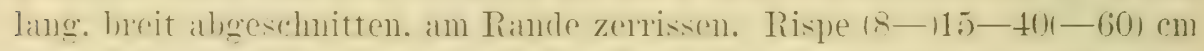
lang. schmal mit zusimmengezgenen ïsten. seltener breit mit abstehenden

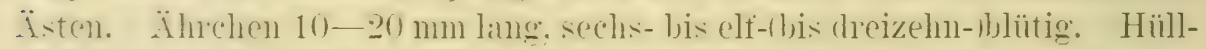
spelzen ungleich, eimnervig. häntig. stumpf. untere $11-12-2.5$ mm. obere 
(2,5-13) nm lang. Deckspelzen stumpf, 3,5-4,5 mm lang, mit kräftigen, rauhen Nerven. Vorspelze so lang wie die I)eckspelze. Antheren 1(-2) mm lang, gelb, selten violett. Blüte Jumi und Juli, später seltener.

An quelligen Orten, an Ufern und Gräben auf nährstoffreichem Boden im ostlichen Gebiet nicht selten bis häufig, im Westen selten und bisher nur bei Itzehoe: Brunsbüttel!!, Heide: Schalkholz bei T'ellingstedt (Prahl 1887!), Husum: Ahrenviöl und Immenstedter Holz (A. Christiansen!) sowie auf den Nordfresischen Insehn anf sylt: Klamshörn bei Archsum und nördlich von Kampen 1912!!.

Die Art wurde erst spät im Gebiete nachgewiesen, und zwar durch IT. Sonder. Seine Beobachtung fand an mehreren stellen Aufnahme, und zwar zunäehst durch Liebmamn (Flora Danica t. 2465 [1849]\}, sodamn dureh Reichenbach (Jc. Fl. Germ. et Helv. I. 48 [1850]; einziger Standort: Oldesloe). - Er selbst gibt die Notiz der Art als G. flutans $\delta$. obtusiflora (Fl. Hamb. 57 [1851]). Hübener erwähnt sie (Fl. v. Hamb. $500[18+7]$.

\section{Formen.}

1. Rispe mit reichverzweigten Ästen; untere Äste mit (ein bis) zwei his vier grundständigen Zweigen; Pflanze bis $1 \mathrm{~m}$ hoch:

var. normalis nov, var. - Verbreitetste Rasse.

Dazı gehören als abweichende Formen:

f. acuminate A. u. Gr. Syn. Mitt.-Europ. Fl. II. 1. 449 (1900). - Äste verlängert, schlaff, etwas überhängend. -Pinneberg: Flottbeker Park (Kausch!). - Übergänge an schattigen Orten zerstreut.

f. glauca nov. f. - Rispenäste sehr verlängert, bis $16 \mathrm{~cm}$ lang, auch nach der Blüte mehr oder weniger abstehend. Rispe 40-60 cm lang; ganze Pflanze schwach graugrün. Anscheinend eine ziemlich selbständige Form. - Angeln: am Fuße der quelligen Strandhöhen von Langballigholz $1911 ! !$

- Rispe mit armährigen Ästen; mitere Rispenäste ohne oder mit einem grundständigen Zweige, öfter einährig; Pflanze $0,1-0,4 \mathrm{~m}$ hoch. aufrecht oder bogig aufsteigend, etwas ins Graugrüne gefärbt.

var. triticee M. T. Lange in Lange Haandb. Dansk. Fl. 2. Udg.

78 (1856). - Sehr zerstreut. Lanenburg: Techin, Lassahn. Büchen, Fitzen, Grambek, Güster, Göttin, Börnsen!!. Stormarn: Frauenholz!!. Husum: Ahrenviöl (A. Christiansen!). Sylt: Klamshörn und Kampen!!.

Dazu als Unterform:

f. minor Lange Bot. Tidsskr. XIV. 77 (1884). - Pflanze nur $0,1-0,2 \mathrm{~m}$ hoch; Ährchen wenigblütig, in einfacher 
Traube. - Auf trockenem Boden selten. Lauenburg: Techin, Lassahn und Grambek!!. Husum: Ahrenviöl (A. Christiansen!).

Die Zuweisung der var. triticea zu einer der beiden Arten ( $G$. fluitans und G. plicata) ist außerordentlich schwierig: Mehrere Merkmale hat die Rasse mit $G$. fluitans gemein. Dahin gehört die Rispenbildung; die Äste sind wenigährig wie bei der genannten Art und besitzen oft nur einen, fast ebenso oft überhaupt keinen grundständigen Zweig. Mehr als drei Ährchen besaß kein einziger der unteren Rispenäste, während $G$. plicata var. normalis deren in der Regel wenigstens doppelt so viele aufweist. Zwar sind die Äste nicht so einseitig gestellt wie bei G. fhitans, doch auch nicht so gleichmäßig allseitig verteilt, wie das oft bei $G$. plicata der Fall ist. Die Antheren, welche bei G. plicata var. normalis nur ganz ausnahmsweise länger als $1 \mathrm{~mm}$ sind, erreichen bei var. triticea in der Regel etwa $2 \mathrm{~mm}$ Länge und weisen dadurch wie auch durch ihre in der Regel (stets?) violette Färbung auf $G$. fluitans hin. Typische G. plicata mit violetten Staubbeutelı scheint sehr selten; von Kiel lag diese Abweichung ror, gesammelt von W. Christiansen!.

Im Gegensatze zum bisher Bemerkten deuten die stumpfen Deckspelzen auf G. plicata. Auch die Deckspelzenlänge, die bei Gräsern oft sehr konstant ist oder nur innerhalb enger Grenzen schwankt, entspricht derjenigen von G. plicata. Sie differiert sehr wenig von $4 \mathrm{~mm}$, während die Deckspelzen von G. fluitans selbst an Kümmerformen nicht unter $5 \mathrm{~mm}$ Länge herabgehen.

Würde man die Form auf Grund der Rispenbildung und der Staubbeutellänge und -farbe zu G. fluitans stellen, dam würde sie im Formenkreise dieser Art mit var. poiformis Fries Nov. Mant. II. 7 (1839) zusammenfallen, welche Form manche Autoren unter G. plicata stellen, während andere G. triticea unter G. Aluitans fassen. Diese Unsicherheit scheint darin ihren Grund zu haben, daß $G$. poiformis Fries und $G$. triticea MI. T. Lange identisch sind. Eine der Friesschen Diagnose entsprechende Abart von G. fluitans kommt z. B. bei Hamburg trotz der Angabe durch Sonder (a. a. 0.57 [1851]) nicht vor, wenn man darunter nicht $G$. triticea verstehen will.

Die auf Sylt beobachtete G.plicata ist var. triticea. Sie wächst bei Kampen ausschließlich, bei Klamshörn mit G. fluitans zusammen, ohne daß G. plicata var. normalis vertreten ist. Nur durch genaue Prüfung der Spelzenlänge wie der Antherengröße (violett, aber nur etwa $2 \mathrm{~mm}$ lang) findet man die Rasse hier zwischen $G$. fluitans heraus. Das deutet auf enge Beziehungen zu G. fuitans hin.

130. (sp. 51). Glyceria nemoralis Uechtritz u. Körnicke Bot. Zeit. XXIV. 121 (1866).

4. Grundachse lockerrasig. Stengel aufrecht oder aufsteigend, 0,3-0,8(-1) m hoch, ziemlich schlaff, glatt, von den übereinander greifenden Scheiden fast ganz verdeckt. Blattscheiden meistens mehr oder minder ranh. Blattfläche $3-7(-10) \mathrm{mm}$ breit, unterseits schwächer, oberseits stärker rauh. Blatthäutchen der unteren Blätter 3-5 mm lang, der oberen 


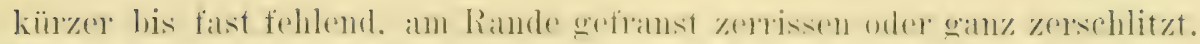

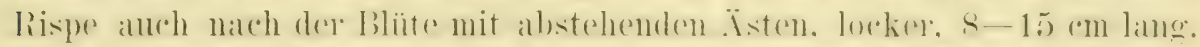
ت̈hrchen 5 $91-10) \mathrm{mm}$ lang. rier- bis zohublütig. Hällspelzen büntig. rimererig. stumpt. untere $1,5 \mathrm{~mm}$, obere $2 \mathrm{~mm}$ lang. Iteckspelzen geaen :3 nmm lang. stumpt, mit drei his vier kiattigen, his zur spitze verlaufenden und drei damit abwechselnden, schwächeren, im oberen $2 / 3$ endenden

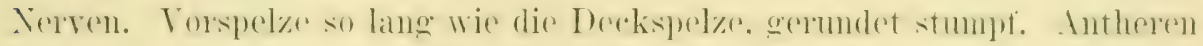
etwa $1 \mathrm{~mm}$ lang, gelb. Blüte Juni bis Juli.

Im südöstlichen Gebiet selir selten.

La uenburg: an den Höhen des Ratzeburger Sees von Dermin bis laak (und Forst Kalkhütte) und bei Waldesruh mehrfich 1912!! (veregl. Verhandl. Bot. Ver. Brandenb. LV. 34 fi. [1913]). Stormarn: in einer quelligen Taldschlucht zwischen Tralauerholz und Frauenholz bei Reinfeld $1913 !$ !.

Die östliche Art ist hier bei Ratzeburg und Reinfeld weit westwärts vorgeschoben.

\section{(s). $49 \times 50$. Glyceria fluitans $\times$ plicata $=$ G. intersita Haushin.}

Nitt. Geogr. Ges. Thüur. III. 230 (1885).

4. Unterscheidet sich von $G$. fluitans durch kürzere, stumpfere, t-5 mm lamge Deckispelzen, durch kïrzere, 1.5-2 mm lange Antheren sowie durch reichere Terzweigung der fast allseitswendigen Rispe. von (i. plimtn durch schwächere Verzweigmg der Rispe. etwas verlängerte Deckspelzen und violette, bis $2 \mathrm{~mm}$ lange Antheren.

Unter den Eltern stellenweise, wahrscheinlich nicht selten. - Lanenburg: Roseburg!!. Lübeck: Gr. Sarau und Rotenhusen!!. Lütjenburg: Behrensdorf!!. Kiel vielfach (A. Christiansen!)!! Rendsburg und Husum mehrfach (A. Christiansen!). Angeln: Ulstrup!!. Sundewitt: Gravenstein!! Ha dersleben: mehrfach (A. Christiansen!).

\section{Formen.}

f. macrostachys A. Christiansen nov. f. - Ährehen bis $4 \mathrm{~cm}$ lang, bis 15́blütig. - Kiel: Meimersdorfer Moor (A. Christiansen 1909!).

\section{Mißbildungen.}

f. m. vivipara nov. f. - Ährchen in Laubsprosse auswachsend. - Kiel: Mronkeberg!. Husum: (Jster-Ohrstedt! und Had ersleben: Eisbüll! (A. Christiansen).

131. (*1). 5\%). Glyceria aquatica Wahlenherg Fl. (Tothob. 1R (1s:0). 4. Grundachse gestreckt, mit verlängerten Ausläufern. Stengel 0,6-1.s m hoch. autrecht, glatt. Blattscheiden eng. glatt. Blattfliche 
Interseits glatt, oberseits und am Rande rauh, $(4-18-10(-15) \mathrm{mm}$ breit. Blatthäutchen $1-3 \mathrm{~mm}$ lang, breit abgeschnitten. Rispe $10-20(-40) \mathrm{cm}$ lang, reichährig, mit zahlreichen, aufrecht abstehenden Ästen. Ährchen 5-6(-8) mm lang, drei- bis sechs- (bis acht-)blütig. Hüllspelzen ungleich, läutig, stark eimnervig, stumpf. untere 2-2,5, obere $3 \mathrm{~mm}$ lang. Deckspelzen etwa $3 \mathrm{~mm}$ lang. stumpf, mit sieben bis neun abwechselnd stärkeren. längeren und schwächeren, kürzeren Nerven. Blüte Juni bis August.

An Ufern, in Gräben und Sümpfen häufig; auch auf Föhr (Schiötz)!!, dagegen auf Sylt zwar genannt (Hansen nach Knuth Bot. Wand. 114 [1890]), aber unsicher (ron Knuth Fl. Nordfr. Ins. nicht wiederholt).

Weber nennt das Gras als Poa aquatica (Prim. Fl. Hols. 7 [1780]).

\section{Formen.}

Vom Typus weicht ab:

f. umbrosa nov. f. - Ährchen mit grünen Deckspelzen; Rispe locker, mit schlaffen, verlängerten Ästen. - An schattigen Orten zerstreut, besonders in Waldsümpfen.

Die Form bildet einen Übergang nach var, arundinacea Ascherson, die trotz Angabe bei uns nicht rorhanden ist.

\section{Mißbildungen.}

f.m. vicipara nor. f. - Ährehen in Laubsprosse answachsend. - Hamburg: Kl. Grasbrook (Laban 1865!) 。

48. Gattung.

\section{Festuca.}

L. Gen. plant. ed. 5. 33 (1754).

1. Ährchen zusammengedrückt; Deckspelzen gekielt ................ 2

- Ährchen rundlich; Deckspelzen am Rücken abgerundet.............. 10

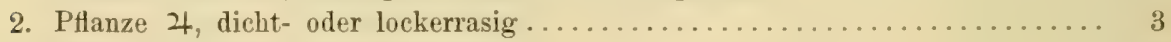

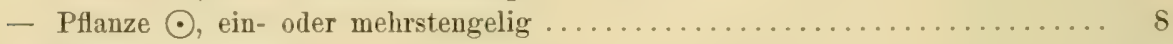

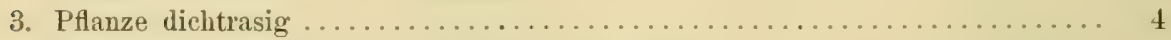

- Pflanze lockerrasig, mit kriechender Grundachse ................ 5

4. Pflanze kräftig; Blätter flach, breit..................... silvatica.

- Pflanze bis $0,5 \mathrm{~m}$ hoch; Blätter gefaltet................ ovina.

5. Granne länger als ihre Deckspelze .................. gigantea.

- Granne kürzer als ihre Deckspelze oder fehlend ................ 6

6. Grundblätter gefaltet; Blätter mit zwei stengelumgreifenden Öhrchen an der

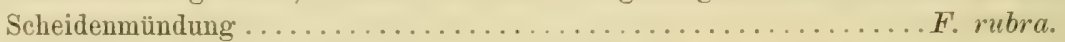

- Grundblätter flach; Öhrchen nicht vorhanden................. 7 
7. Kispe mit nach der Blüte aufrechten Ästen; unterster Ast mit bis sechs Ährchen und $0-1(-2)$ grundständigen, bis zweiährigen '/weigen.......F. pratensis.

- Rispenäste schlaff, überhängend; unterster Ast mit bis 20 Ährchen und 0-2 grundständigen, bis achtihrigen Zweigen .............. arundinacea.

8. Deckspelzen gramnenlos ........................... rigida.

- Deckspelzen langbegrannt

9. Stengel unter der Kispe blattlos; unterster Rispenast etwa halb so grob wie

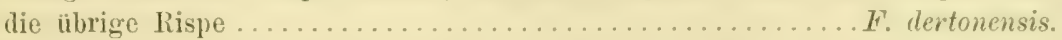

- Stengel bis zur Rispe beblattert; unterster Rispenast mehrmals kleiner als die

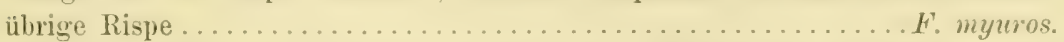

10. Blätter gefaltet; untere Hüllspelze dreinervig; Deckspelzen wenigstens $3 \mathrm{~mm}$ $\operatorname{lang}$

-- Blätter flach; untere Hüllspelze einnerrig; Deckspelzen höchstens 2,5 $\mathrm{mm}$

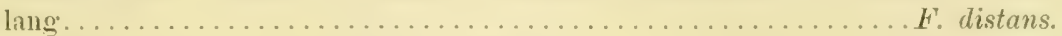

11. Deckspelze mit fünf undeutlichen Nerven ............... thalassica.

- Deckspelze mit drei stärkeren und zwei schwächeren Nerren....F. angusta.

132. (s). 531. Festuca distans Kimth Enumeratio I. 393 (1833).

4. Grundachse dichtrasig. Stengel aufrecht oder aufsteigend, (0.1-0.5 m hoch, glatt. Blattscheiden glatt. weit abwäts often. Blattflatche dünn, in der Regel flach. nur am Rande und am Vitteherr schwach raul. Blatthäutchen 1-2 mm lang, breit abgeschnitten. Rispe $5-10 \mathrm{~cm}$ lang, mit nach der Blïte zurückgeschlagenen Ästen; untere Äste mit zwei oder mehr grundstiandigen Zweigen. Ährehen 4-5 mm lang, drei- bis sechs-(bis acht-bblütig. Hüllspelzen ungleich. untere etwa 1 mm lang. eimnervig. stumptlich, obere gegen $2 \mathrm{~mm}$ lang. dreinerrig, oft spitzlich. I)eckspelzen 2( 2.5) mm lang. stumpf, am Rücken abgerundet, schwach oder dentlich fünfnervig. Frucht frei. Blüte Mai bis Oktober.

Auf Salzboden an der Nord- und Ostsee häufig bis zerstrent, an der Trate rom Treidelstieg (Friedrich!)!!. an der Elbe ron Brumsbïtel!! ab)wärts, anf den Nordseeinseln wenig rerbreitet, und zwar anf Helgoland: Cnterland (Hallier) früher, Hallig Oland (Buchenau), Föhr (Schiötz) verbreitet!!. Amrum: Norddorf!!, sylt (Schiötz): Eidumer Togelkoje, zwischen Keitum und Archsum!! und Röm: Ture t.Taap). Sonst nur auf schutt und an Wegen vorübergehend verschleppt, und zwar:

Lauenburg (Nolte nach Klatt als gesehen; ,Lauenburgia. leg. Hornemam" im Hb. Kopenhagen!) Hamburg: zwischen Blankenese.

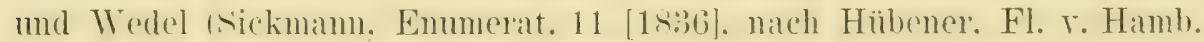
500 [1847] unrichtig. aber von Sonder. Festschr. Jaturt.-Ters. Hamb. 130 [1:76]. ansdrücklich unter den rerschleppten salzptlanzen erwähnt). (ieorgswärder (Zimpel 1893 !). Teddel! und steinwäirder (.J. Schnidt) ।.. Elbinseln" schon nach sonder. Hb. Lübeck!). Hammertorook (Sonder. Zimpel usw.!!. st. Georg (C. Timm. Laban!!. Mühlenkamp in Winterhude Lalbanı. mehn-

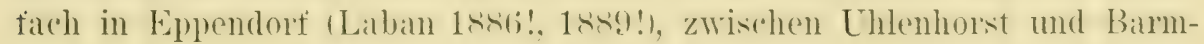


beck! sowie zwischen Bambech und Alsterdorf ILabanl. Steilshop!!. st. Panli sonder. (. Timmı, in Altona bei der Holstenluanerei IZimpel

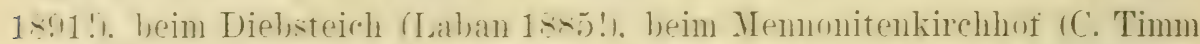
1s: nicht selten (Friedrich), Oldenburg i. H.: in Großenbrode!!. Kiel:

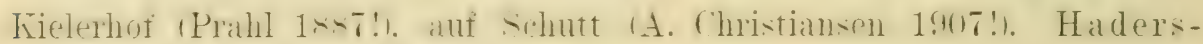
leben: Skrydstrup (Prahl).

Anf Salzboden des Bimenlandes bei Oldesloe: bei der Saline

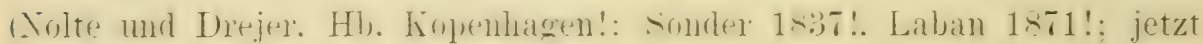
rerschwunden), im Brenner Moore (Ch. Sonder) 1908, 1912!!.

WVeber erwähnt die Pflanze als Poa satina (Prim. Fl. Hols. Suppl. $2[1787])$.

Hübeners Angaben (a. a. 0. 500) für Langenfelde, Sülldorf, Osdorf und Schenefeld sind zweifelhaft. Nach C. Weber (Naturw. Ver. Schlesw.-Holst. IX. 2. 206 [1893]) elscheint $F$. distans selten im Grasland der eingedeichten Marsch.

\section{Formen.}

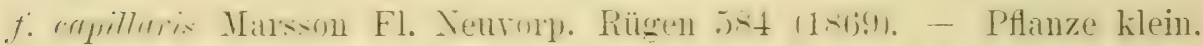
sehr dichtrasig; Stengel etwa $0,1 \mathrm{~m}$ hoch; Blätter schmal, gefaltet; Rispe schmal (fast ährenförmig): Äste zur Blittezeit wenig abstehend. -

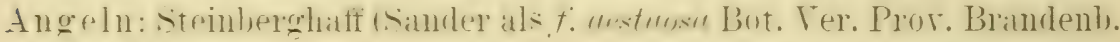
VI. 231 [1864]). Hadersleben: Aarösund und Sliepminde (Prahl).

133. (s). 5t). Festuca thalassica Kimnth lievis. (iram. I. 129 (18:!).

4. Grundachse mit zahlreichen, oft stark verlängerten, unfrucht-

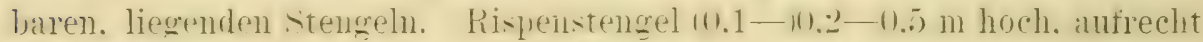
order anfstrigend. glatt. Blattscheiden glatt. Blattflar he dicklich. in der Reger grefaltet. glatt oder oifter an Rande oberwärts rauh. Blatthäutchen 1-2 $\mathrm{mm}$ lang, breit gestutzt. Rispe $6-15 \mathrm{~cm}$ lang, mit in der Regel autrechten his wenig abstehenden Ästen: mutere ïste mit meistens einem. zuweilen aber nehreren. grundständigen \%weigen. Ährhen $4-10 \mathrm{~mm}$

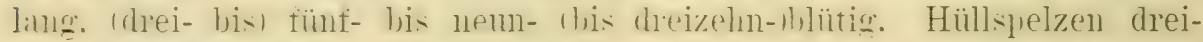

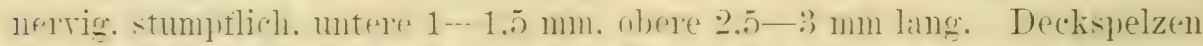

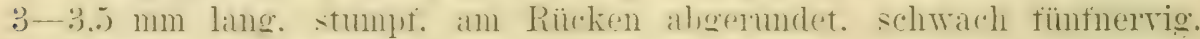
Frucht frei. Staubbeutel etwa $2 \mathrm{~mm}$ lang. Blïte Juni bis September.

Auf Salzboden an der Ostsee nicht selten, an der Nordsee häufig,

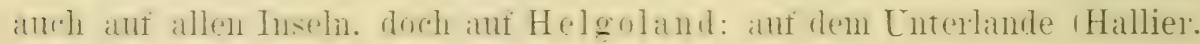
Dalla Torre) früher, jetzt verschwunden.

Die Art findet aus dem Gebiete die erste Erwähnung durch Hornemann (Flora Danica t. 1626 [1819]). 
Das Gras ist an stärkeren Salzgehalt des Bodens gebunden als $F$. distans und fehlt daher bimenländischen Örtlichkeiten (auch bei Oldesloe). Von der unteren Elbe erwähnt die Flora Danica allerdings Glückstadt als Standort, doch ist die Richtigkeit dieser Angabe sehr zweifelhaft, da die nicht obligaten Salzpflanzen $F$. distans und Juncus Gerardi bereits bedeutend weiter elbabwärts bei Brumsbüttel ihre obersten Vorkommen besitzen. Die Notiz bei Mößler (Handb. Gewächsk. 120 [1815]), derzufolge Poa maritime $=F$. thalassica an der Elbe bei Schulau vorgekommen sein soll, bezieht sich höchstwahrscheinlich auf $F$. distans (vgl. diese), zu welcher Art wahrscheinlich auch Poa maritima Oeder (Flora Danica t. 251 [1766]) zu stellen ist.

\section{Formen.}

Vom Typus weichen ab:

f. anomale Lange Haandb. Dansk. Fl. 3. Udg. 86 (1864). Rispenäste kurz, einährig, Rispe daher fast ährig; Blätter fein, schmal. - Amrum (Nolte 1825, Hb. Kiel!). Sylt (Bargum 1812, Hb. Kiel!).

Lange nannte die Form (anscheinend unter Ausscheidung monströser Formelemente) $f$. nana (Bot. Tidsshr. XI. 93 [1879]). Seine Diagnose an dieser Stelle weicht von der zur Abbildung der Form (Flora Danica t. 2823 [1864]) gegebenen Beschreibung: „humilior, panicula subspicata, spiculis majoribus, subsolitariis, brevissime pedunculatis, saepe viviparis, palea inferiore acuminata" durch das Fehlen der beiden letzten Bestimmungen ab, wogegen die Blattbeschaffenheit hervorgehoben wird. Als Angabe hat die Flora Danica: "Amrum. (Nolte)".

f. amethystina G. F. W. Meyer Chlor. Hannov. 629 (1836). Deckspelzen lebhaft violett. - So zerstreut, besonders an der Ostsee.

134. (adv. 71). Festuca angusta A. 11. (i1. Syn. Mitt.-Eurol. Fl. II. 1. 460 (1900).

4. Grundachse mit zahlreichen, aufrechten, kurzen, unfruchtbaren stengeln. Rispentragende Stengel 0,2-0,5 m hoch, anfrecht, glatt. Blattscheiden glatt, eng. Blattfliche $11-2) \mathrm{mm}$ breit. etwas starr, eingerollt. gegen die Spitze rauh. Blatthäutchen $1 \mathrm{~mm}$ lang, stumpflich. Rispe 8-10(-o) cm lang. mit zusammengezogenen oder schwath aufrecht abstehenden ت̈sten. Ährehen 5- $5 \mathrm{~mm}$ lang, vier-bis sechsblïtig. Hüllspelzen stumptich oder spitzlich, dreinervig. untere etwa $1.5 \mathrm{~mm}$, obere etwa $3 \mathrm{~mm}$ lang. Deckspelzen etwa $3 \mathrm{~mm}$ lang. fünfnervig. drei Nerven stärker, zwei schwächer. Staubbentel $1 \mathrm{~mm}$ lang. Blüte August und September.

Auf Schutt bei Hamburg selten.

Hamburg: Wollkämmerei am Reiherstieg (J. Schmidt!, Jaap!, Zimpel!). 
$\mathrm{Zu}$ dieser Art gehören Eatonia pennsylvanica in Deutsche Bot. Monatsschr. XVI. 115 (1898), Glyceria tenuifolia a. a. O. XVII. 125 (1899), Festuca convoluta a. a. 0. XVII. 125 (1899) und F. thalassica Zimpel Herb. z. 'T.!.

135. (s1. 5)). Festuca ovina I. Sper. plant. ed. 1. 73 (1753) snbsp. eu-ovina Hackel Nonogr. Festuc. 85 (1882).

4. Meistens sehr dichtrasig. Stengel in der Regel aufrecht, starr oder stwas schlaff. $0.1-0.6 \mathrm{~m}$ horh. glatt. Blattscheiden ganz offen oder

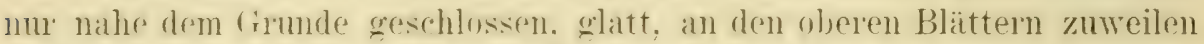
schwarh erweitert. Blattlärhe glatt oder olerwäts schwach, selten stärker raulı, bonstenförmigr grafaltet. nur ausnalımsweise an den sitengelblättem nireht viollig grafaltet. Blatthäutchen mit zwei den sitengel umfassenden. etwa $1 \mathrm{~mm}$ langen Lappen, sehr kurz. Rispe dïmnästig̣, 3) bis 10 con lang. mit zusammengezogenen oder wenig aufrecht abstehenden Asten. Ihrchren 3-81-10) mm langr. drei- his arohthlütig. Hüllspelzen schmal. spitz. obere dreinervig. -2 - 2.5) mm lang. untere (einnervig. 1,5) bis 2 mm lang. I)echspelzen gekiolt. grammonlos orler moistens begrannt, 3-3.5) mm lang. spitz. kahl oder behatat. undeutlich fünfuervig. Fruchtknoten kahl. Antheren etwa $2 \mathrm{~mm}$ lang. Blïte Mai bis Juli, zuweilen später.

Auf trockenem bis mäbig fourhtem Boden auf Heiden. Mooren. Triften, in Wäldrm, febüschen und an Abhängen häufig, weniger in sïmpfen; anch auf den Nordfriesischen Inseln verbreitet.

WVeber nennt die Art unter Nr. 82 und 84 seiner Flora (Prim. Fl. Hols. $8[1780])$.

\section{Formen.}

1. Blattfläche $0,3-0,6 \mathrm{~mm}$ dick, fadenförmig .......... 2

- Blattfläche $0,7-1,2 \mathrm{~mm}$ dick, derb, starr........... 3

2. Deckspelzen grammenlos; Rispe kurz, schmal, mit aufrecht anliegenden, wenigährigen Ästen:

var. capillata Hackel Monogr. Festuc. 85 (1882). - Eine sehr charakteristische Form, bisher nur im Süden und Westen des Gebiets gefunden. - Lauenburg: Ziehnburger Schleuse (J. Schmidt und Zimpel 1898!), Roseburg!!. Stormarn: Ahrensburg (Prahl 1891!) am Ahrensfelder 'Teich!!. Hamburg (Sonder): Hasselbrook in Hamm (J. A. Schmidt usw. 1868!), Barmbeck (J. A. Schmidt 1872!, 1873!), Borsteler Moor (J. A. Schmidt 1872!), Eppendorfer Moor (J. A. Schmidt 1870!)!!, Langenholn 1906!!, Wandsbek (Kausch 1895!), Georgswärder (Kausch 1894!). Pinneberg: Langenfelde (Röper 1908!), Flottbek (W. Hansen 1886!, Laban 1890!). 
Einige weitere Angaben sind unsicher, so die von Sylt: Wenningstedt und List (Naturw. Ver. Schlesw.-Holst. XIII. 1. 37 [1905]), die von Föhr: Südstrand (Knuth Fl. Nordfries. Ins. 148 [1895]) und die durch Nolte gegebene Notiz (Nov. Fl. Hols. 13 [1826] als F. temifolia). Letztere Angabe grünlet sich auf Noltesche Pflanzen aus Eiderstedt. welche Knuth (Fl. v. Schlesw.-Holst. 775 [1857]) wieder als F. tenuifolia aufführte, trotzdem die betr. Exemplare Grannen besitzen!, ebenso wie andere Pflanzen, die Nolte in Lauenburg sammelte!. Die älteste Liternturangabe findet sich für unser Gebiet bei Hornemann (Oec. Plantel. 3. Udg. 1. 115 [1821]) (ob richtig?). Sichmann nennt sie für Hamburg (Enumeratio 11 [1836]); ron hier bezeichnet Sonder sie (Fl. Hamb. 61/62 [1851]) ungenauerweise als Schattenform.

Die Varietät zerfällt in drei Formen:

f. typica nov. t. - Stengel 0,25-0, $40 \mathrm{~m}$ hoch, wenigstens doppelt so lang wie die Blätter. - Stormarn: Ahrensburg (Prahl!), Ahrensfelder T'eich!!. Hamburg: Eppendorfer Moor (J. A. Schmidt!)!!, Borsteler J Joor (J. A. Schmidt!), Langenhorn!!, Georgswärder und Wandsbek (Kausch!). Pinneberg: Flottbek (Hansen!). f. Frisia A.u. Gr. Syn. Mitt.-Europ. Fl. II. 1. 467 (1900). Stengel 0,1-0,2 m hoch, wenig länger als die schlaffen Blätter. - Hamburg: feuchte, moorige Triften beim Hasselbrook (J. A. Schmidt!, Laban! 1868).

f. arenaria nov. f. - Stengel $5-15 \mathrm{~cm}$ hoch, beträchtlich länger als die kưzen, starren Blätter. - Auf trockenem Sandboden. Lauenburg: Ziehnburger Schleuse (Zimpel!), Roseburg!!. Hamburg: Barmbeck (J. A. Schmidt!), Hamm (C. Timm!). - Eine Form mit ziemlich derben, breiten Scheiden, der var. glauca etwas angenähert, sammelte beim Hasselbrook Laban 1868 !.

- Deckspelzen mit 1-2 mm langer Granne; Rispe lockerer, breiter, mit aufrecht abstehenden, mehrährigen Ästen:

var. vulgaris Koch Syn. Deutsch. u. Schweiz. F1. ed. 1. \$12 (1837). - Häufigste Rasse. Sie zerfällt in zwei Formen mit mehreren Unterformen:

1. f. gemina Godr. u. Gren. Fl. France III. 570 (18526). Pflanze renig kräftig, dünnstengelig; Ährchen $4-6 \mathrm{~mm}$ lang. - Häufig. Dazu als Unterformen:

f. sciaphita Schur Enum. Plant. Transs. 787 (1866). Deckspelze am Rücken rauh, an den Rändern gewimpert.-Lauen burg: Sachsenwald(Zimpel!). Stormarn: Wandsbek (Zimpel!). Segeberg: 
Bramstedt!! Kiel mehrfach (A. Christiansen!). Schlestrig: Tiergarten (Himichsen (1852!).

f. Terifolia Hackel Monogr. Festuc. 87 (1882). Stengel und Blätter glatt (oder letztere nahe der Spitze sehr schwach rauh). - Stormarn: Bramfelder Heide (J. A. Schmidt 18it!), Brenner Moor bei Oldesloe!!. Oldenburg: Dahme. Heiligenhafen!!. Itzehoe: Nenendeich!!.

f. umbrosu Hackel a. a. 0. 87 (1882). - Stengel und Blätter schlaff; Rispe locker; Ährchen grün bis bleichgrün. - Nicht selten in Wäldern und Gebüschen. - Zu dieser Form resp. in ihre Nähe gehören anscheinend Formen unseres Gebiets mit gelbgrünen Ährchen (so nach C. Timm Naturw. Ter. Hamb. N. F. TT. 85 [1880]) und mit gelben Antheren ( $f$. flacescens A. Christiansen in sched.?). f. subarenaria nor. f. - Stengel etwa $10 \mathrm{~cm}$ hoch, kaum länger als die Blätter; Rispe dicht, kurz. mit kurzen, wenigährigen Ästen. - Lauenburg (Nolte!). Eiderstedt (Nolte 1825!). Föhr 1913!!. Anch sonst zerstreut. - Im Habitus erimnert die Abart an $f$. arenaria.

2. f. Lemeni A. u. Gr. a. a. O. II. 1. 468 (1900).Pflanze 0,4-0,6 $\mathrm{m}$ hoch, starrstengelig; Ährchen 6-8 mm lang. - Selten. Stormarn: Hahnheider Berg bei Trittau (J. A. Schmidt 1870!).

3. Pflanze umbereift; Blattscheiden der Grundblätter wenig derb. ziemlich schmal:

var. duriuscula Koch Syn. Deutsche u. Schweiz. Fl. ed. 1.812 (1837). - Nicht selten. - Unsere Pflanze ist $f$. gemina Godr. Fl. Lorr. III. 172 (1844) mit 0,2-0,4 m hohem Stengel, bis $10 \mathrm{~cm}$ langer Rispe und $7-8 \mathrm{~mm}$ langen Ihrehen. Dazu als Unterformen:

f. Teiantha Sickmami Enumeratio 12 (1836). - Deckspelzen kahl. So nicht selten. - Hierher als wenig wichtige Abart f. Tongifolia Thuill. Fl. Par. ed. ¿. 50 (1799) mit verlängerten Blättern, mehrfach im Gebiet beobachitet.

f. villosa Schrader Fl. Germ. I. 320 (1806). - Deckspelzen behaart. Srnonym ist $f$. dasyantha Sickmann a. a. O. 12 (1836). - Selten. Lauenburg: nahe der Grenze bei Horst!! Hamburg (Sickmann). 


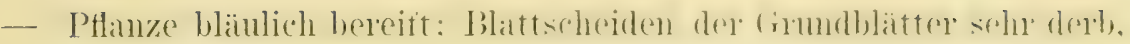
breit, bleich:

var: glaucu Hackel Monogr. Festuc. 97 (1882). - Im Elbgebiet sowie an der Nord- und Ostsee auf Sandland und Dïnen rerbreitet. Zerfällt in zwei Formen:

1. f. genuina Hackel a. a. 0. 94 (1882). - Pflanze niedrig, bis $0,3 \mathrm{~m}$ hoch; Rispe kurz, dicht, mit geraden Ästen; Ährchen 7-8 mm lang. - Häufigste Form der Rasse. Dazu als Unterform:

f. cinerea Hackel a. a. 0.95 (1882). - Deckspelzen behaart. - Neustadt: Grömitz!!.

2. $f$. pallens Hackel a. a. 0.95 (1882). - Pflanze 0,3-0,4 m hoch; Rispe rerlängert, bis $10 \mathrm{~cm}$ lang, lockerer, mit geschlängelten Ästen; Ährchen 7 -8 mm lang: - So besonders im Elbgebiet, sonst sehr zerstreut und nur in Übergängen nach f. genuma. In einer solchen Ubergangsform auch als $f$. puberula Hackel a. a. 0.95 (1882) bei N e us ta d t: Grömitz!!(Deckspelzen behaart). Zuweilen sind bei $f$. pallens die Blattflächen röllig glatt, so bei Pflanzen ron Hamburg: Geesthacht 1877! und Stormaru: Boberg 1881! (J. A. Schmidt).

Verwandt ist mit $f$. pallens, aber durch nur 5-6 mm lange Ährehen und niedrigeren Wuchs geschieden, $f$. caesia Hackel a. a. 0.95 (1882), beobachtet in Übergängen nach $f$. gemuina bei Hamburg: Bergedorf (J. A. Schmidt 1864!).

Festuca vallesiaca Schleicher in Gaudin Fl. Helr. I. 242 (1811) fehlt bei uns. Die erste Notiz gab für unser Florengebiet C. Timm (Naturw. Ver. Hamb. N. F. IV. is [1880]) für Hamburg: an der Lagerstrabe in St. Pauli; es wird sich um $F$. ovina var: glauca gehandelt haben. Knuth wiederholt die Angabe (Fl. ₹. Schlesw.-Holst. 775 [1887]) mit der Hinzufügung; daß die Art bier und da rorkomme. Er nennt sie auch für Helgoland als ron Brody beobachtet (als $F$. sulcate Hackel in Allg. Bot. Zeitschr. IT. 110 [1898]); die betreffende PHanze gehört aber nach Ascherson zu $F$. rubra.

Festuce heterophylla Lam. Fl. Franc. ed. 1. 600 (1778) gehört ebenfalls trotz vielfacher Aufzählung der Flora unseres Gebiets nicht an. Die älteste Angabe steht bei Buek (Hoppe Bot. Taschenb. 92 [1801]) als F. nemorum. Yahezu alle spiteren Hamburger Floristen nemmen die Art, so Sichmann (Enumeratio 12 [1836]), Hübener (Fl. r. Hamb. 503 [1846]; Exemplare im Hb. Lübeck $=F$. ovina vulgaris umbrosa!), Sonder (Fl. Hamb. $62[1851]$; ron Bambeck [Hb. Kỉel] $=$ F. rubra!, ron Eimsbüttel [Hb. Lïbeck] $=$ F. rubra!) und Laban (Fl. r. Hamb. 1.-4. Aufl.; 4. Aufl. 204 [1857]). Sondersche Pflanzen erhielt J. Lange, der die Art danach abbildete (Flora Danica t. 2645 [1861]); Ausläufer fehlten nach der Abbildung, auch bemerkt die Diagnose „.enespitosa“. Ob aber die Stolonen wirklich röllig fehlten? Das Exemplar sah ich nicht; unter den Stücken des Kopenhagener Herbars fehlt es. Da aber andere Pflanzen Sonders zu F. rubra gehören, so 
bleibt die Art trotz der Flora Danica zweifelhaft, um so mehr, als Sonder sie in der

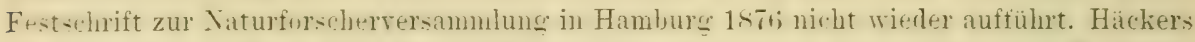
F. heterophylla (Fl. v. Lüb. 43 [1844]) ist teils F. mbra!, teils (nach Lenz Mecklenb. Archir XXII. 86 [1869]) $F$. avina duriuscula. Weitere neue Standorte brachten in der Literatur Hallier (Bot. Zeit. XXI. Beilage 8 [1863]), Lienau (Fl. r. Eutin 3 [1863]), Klatt (Fl. v. Lauenb. 165 [1865]) und Prahl (Beiträge Fl. Schlesw. 147 [1872]). Alle Notizen sind unbestätigt geblieben resp. zurückgenommen (Prahl) worden. Alle sonstigen Ver-

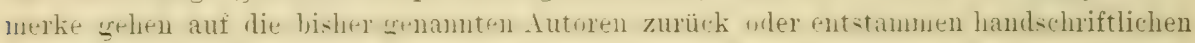
Verzeichnissen, so bei Borchmann, Laban, Hennings, von Fischer-Benzon-Steinvorth und Knuth. Eingesehene Belege ron Kohlmeyer!, Laban! und J. A. Schmidt! gehören zu F. rubra, wohin wohl auch F. heterophylla bei Reichenbach (Vorl.-Verz. Hamb. 20 [1881]) zu stellen ist.

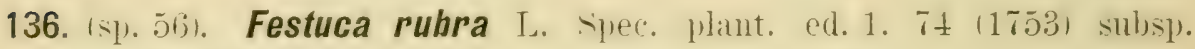
eu-rubra Hackel MIonog1. Festuc. 138 (1882).

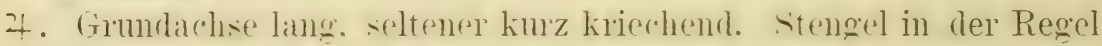
aufrecht. star oder sohlaff. (1).15-10.3-0.8 m hrorlı. olatt. Blattscheiden geschlosien. eng. glatt. Blattfläche der frumbläter meistens gefaltet. der stengelblatter mristens Hach. beidereits lauh. Blatthäutchen mit ren stengel mogreitenden. kihlen. etwa $1 \mathrm{~mm}$ langen öhrehen. Rispe zienlich locker. $4-12 \mathrm{~cm}$ lang, mit längeren, etwas srohlatfen ästen. sitener zusammengezogen. mit küzeren. starelen Asten. Ährchen $(6-1$ r-12 mm lang. Idrei-his riel- his serhshlütio. Hüllsuelzen schmal, spitz,

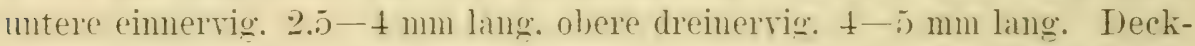
spelzen 14.5- 5 molentich generrt. Fruchthnoten kahl. Antheren : -4 mm lang. Blüte Juni bis August, seltener später.

Auf Wiesen und Triften. in Gebüschen mo Wäldern. an Abhängen mul Wegrändern. weniger in Mooren und sïnuten. häutig: auch auf den Sordfriesischen Inseln und Helgoland.

Zuerst genannt von Weber (Prim. Fl. Hols. 8 [1780]).

\section{Formen.}

1. Pflanze mehr oder weniger lockerwüchsig, mit kurzen bis verlängerten Ausläufern :

var. genuina Hackel Monogr. Festuc. 138 (1882). — Zerfällt. . 2

-- Pfanze tast dichtrasig. mit nur soh kurzen Ausüufern (Sienkem):

var. fallax Hackel a. a. O. 142 (1882). - Eine an F. heteropluylla erinnernde Form, bei uns als:

f. subccespitosa Sonder Fl. Hamb. 63 (1851). - Pflanze nicht völlig dichtrasig, 0,6-0,8 m hoch; Rispe locker, mit verlängerten Ästen. - In Wäldern, Gebüschen und Mooren zerstreut, auch auf $\mathrm{Helgoland}$ (Hallier) und Amrum: zwischen Nebel und Norddorf!!. 
Der Beschreibung nach ist $F$. rubra $\delta$. altissima Hallier Bot. Zeit. XXI. Beilage. 8 (1863) hierherzuziehen.

Dazı als Unterformen:

f. grandiflor Hackel a.a. O. 138 (1882), - Ährehen 10-12 mm lang, länger begrannt. - So z. B. A penrade: Warnitzwig!!.

f. subleteropluylla nov. f. - Stengelblätter mit 3-4 (beim 'Typus - 2) mm breiter Blattfäche. - So z.B. Stormarn: Wandsbek (J.A.Schmidt 1869!; untere Scheiden schwach kurzharig). Hamburg: zwischen Hellbrook und Farmsen (J.A.Schmidt $1871 !)$

2. Deckspelzen kahl oder zerstreut kurzharig .............

- Deckspelzen dicht kurz behaart:

f. arenaria Fries Fl. Halland. 28 (1818). - Ährehen bis $12 \mathrm{~mm}$ lang, Deckspelzen bis 7,5 mm lang; Rispe mit ziemlich kurzen, oft zusammengezogenen Ästen; Stengelblätter gefaltet.

Auf Dünensand an der Nord- und Ostseeküste verbreitet, selbst auf Helgoland: Dïne (schon Nolte!) 1912!!. - Verbreitet sind Übergänge in den Typus.

Zu dieser Abart zählen $F$. ovina $f$. dumetorum bei Hornemann (0ec. Plantel. 3. Udg. 1. 118 [1821]) und F. lirsuta Homemann (Flora Danica t. 1627 [1819]). Ein Exemplar Noltes im Kopenhagener Herbar, bezeichnet „Lauenburg's!, stammt wahrscheinlich aus dem Gebiet der Untertrave, von wo mehrfach Pflanzen mit gleicher Bezeichnung in älteren Herbarien unseres Gebiets sich finden. Eine Angabe für Geesthacht nach Sonder fehlt in Sonders Schriften.

3. Alle Blätter eingerollt oder nur die Stengelblätter flach....... Alle Blätter flach:

f. multiflora A. u. Gr. Syn. Mitt.-Europ. Fl. II. 1. 499 (1900). Stengel derb, hoch; Ährchen zu vielen, über $1 \mathrm{~cm}$ lang.

Selten. H amburg: Bergedorf (J. A. Schmidt!). Segeberg: Stuvenborn (J. Schmidt!). Kiel: bei Raisdorf und bei Kronsbek nahe Lindhöft (A. Christiansen!).

Dazu als Unterform:

f. microstachya nov. f. - Pflanze niedriger; Ährchen nur 7-8 mm lang, mit wenigen Blüten. - Hamburg: Bergedorf (J. A. Schmidt 1866!).

4. Untere Blattscheiden kahl (selten zerstreut huzhaarig)........

- Untere Blattscheiden dicht kurzharig:

$f$. pubescens nor. f. - Eine anscheinend besonders an feuchten Standorten (z. B. in Erlenbrïchen) anftretende Abart. - 
Stormar'n: Steinbeker Moor(J.A.Schmidt1873!), am Ahrensfelder 'Teiche bei Ahrensburg 1906!!, bei der Oldesloer Saline (J. A. Schmidt 1870!). Eutin: Bujendorfer Moor!!. Rendsburg: Methorst (A. Christiansen!).

5. Ährchen in der Regel 7 - $8 \mathrm{~mm}$ lang; Grundblätter mit etwa $0,7 \mathrm{~mm}$ dicker Blattflache, ziemlich schlaff: stengel und Bläter meistens dunkelgrïı . . . . . . . . . . . . . . . . . . . . .

- Ährchen 9-10 mm lang: (rlundbläter $0 . x-1.2 \mathrm{~mm}$ dick. derb, oft starr; Stengel und Blätter meistens graugrün; Grumdachse in der Regel weit kriechend:

f. duriuscula Gaudin Fl. Helv. I. 289 (1828). - Zerstreut. Hamburg: Geesthacht (Kausch 1891!), Bergedorf 1904!!. Pinneberg: Schulau!!. Glückstadt: Ivenfleth!!. Lübeck: mehrfach an der Trave von Herrenwiek abwärts!!. Kiel: Schönberg 1823!, Kolberger Heide 1827!, Friedrichsort 1833 ! (J. J. Meyer), Stein (A. Christiansen!).

i. Deckspelzen kahl:

f. vulgaris Gaudin a. a. O. I. 285 (1828). - Häufigste Form.

Dazu als Unterformen:

f. glaucescens Hackel a. a. O. 139 (1882). - Ganze Pflanze graugrïn, Ährchen graugrün bereift. -.. Itzehoe: Brokstedt, Bargfeld, Hennstedt!!. Flensburg: bei der Kupfermühlenhölzumg!!.

f. megastachys Gaudin a. a. O. I. 287 (1828). - Ährchen 10-12 mm lang; Deckspelzen mit verlängerter Granne. - Zerstreut. Hamburg: Besenhorst 1869!, Rotenhaus!, zwischen Bergedorf und Reinbek!, zwischen Horn und Schiffbek! und bei Winterhude! (J.A.Schmidt). Stormarn: Wandsbek (Zimpel!). Lübeck: Schlutup (J. A. Schmidt!). Kiel: an der Preetzer Chaussee!, Wentorfer Strand! (A. Christiansen). Itzehoe: Wiedenborstel!!. Flensburg: Langballigau (Hansen!), Randershof!!.

f. Titoratis Hackel a. a. O. 139 (1882). - Pflanze weit kriechend; Stengel $15-25 \mathrm{~cm}$ hoch, aus knickigem Grunde aufrecht; Rispe kurz, 2-4 cm lang, mit sechs bis zwölf Ährchen; Rispenäste zusammengezogen; Blätter kurz, steif, dick, rimnig. - An der Nordsee verbreitet; früher genannt: Dithmarschen, Eiderstedt (Weber als neue Form in Naturw. Ver. Schlesw.-Holst. IX. 2. $212[1892])$. 
Hierher (nicht zu f. pascua) dürfte (nach den Standorten) f. trichophylla Knuth (Fl. Nordfries. Ins. 149 [1895]) von Norderoog, Hooge und Nordstrandischmoor zu stellen sein. An der Ostsee ist auf diese Form zu achten.

f. pascu Anderss. Gram. Scand. 20 (1852). - Wie niedrige, kleinrispige Formen des Typus, aber mit eingerollten Stengeiblättern. - 'Trockenform. Hamburg: Rotenhaus (C. Timm Nat. Ver. Hamb. N. F. IV. 85 [1880]). Sylt: Morsumheide!!, Rantum (Kuckuck!). Helgoland (Knuth!).

f. nemoralis Anderss. a. a. 0. 21 (1852). - Stengel hoch, etwas schlaff; Rispe verlängert; Ährehen bleichgrï. An schattigen Orten verbreitet.

- Deckspelzen behaart:

f. barbata Hackel a. a. 0. 139 (1882). - Zerstreut. Hamburg (Sickmann, Sonder). Pinneberg: Nienstedtener Elbufer (C. Timm als f. villosa Koch), Flottbek (Zimpel 1888!). Stormarn: Ahrensfelder Teich!!, Bremner Hoor bei Oldesloe!!. Neustadt: Pelzerhaken (kombiniert mit f. pascua)!!, Bliesdorf!!. Kiel: NIönkeberg! und Bülk! (A. Christiansen). Schleswig: am Schleiufer (Hinrichsen!). Rendsburg: Methorst! und Husum: Arlewatt! (A. Christiansen). Tondern: am Langenberge bei Leck (Prahl!). Helgoland: Oberland zerstreut (Hallier Bot. Zeit. XXI. Beilage. 8 [1863] als B. villosa Koch). Amrum: Nebel!!.

Sickmamn tremnt $f$. villosa und $f$. subrillosa, doch ist kaum ein Zweifel daran möglich, dab der zweite Name Übergangsformen der erstgenannten Abart nach $f$. villosa bezeichnet. Sickmann erklärt seine f. villosa für identisch mit $F$, dumetorum $\mathrm{L}$., die indessen bei uns fehlt. Prahl gebraucht den Namen f. scopulorum Fries? (Krit. Fl. 259 [1890]).

137. (s). 57). Festuca pratensis Hudson Fl. Angl. ed. 1. 37 (1762).

4. Grundachse lockerrasig; ausläufertreibend. Stengel aufrecht oder anfsteigend. (9.3-0.7 m hoch, oberwärts blattlos, glatt. Blattscheiden weit abwärts offen, glatt. Blattflache bis 4 mm breit. flach orler selten eingerollt. beiderseits deutlich, aber nicht stark rauh. am Rande stark raul. Blatthäutchen fast fehlend, olme öhrelen. Rispe $6-15(-20) \mathrm{cm}$ lang, mit außer der Blütezeit aufrechten oder fast aufrechten Ästen. schmal, ihr unterster Rispenast wenigährig (bis sechs), ohne oder mit einem kurzen. ein- bis zweiährigen grundständigen Zweige. Ährchen 8-12 mm lang, fünf- bis acht- (bis 13-)blütig. Hüllspelzen spitz, ungleich, untere eimervig; bis $2,5 \mathrm{~mm}$ lang, obere dreinerrig, bis 4 mm lang. Deck- 
spelzen bis $6.5 \mathrm{~mm}$ lang. undeutlich fünfnervig, kahl, grannenlos, oberwärts hautrandig. Fruchtknoten kahl. Blüte Juni bis August. einzehn später.

Auf Wiesen, an Ufern und Wegen, in Gebüschen und an Waldrändern häufig, doch auf den Nordfriesischen Inseln nur auf Helgoland (Hallier. Brody!. auch $f$. sulwpirentu A. u. (ir. nach Ascherson, Brody!). Föhr (schiotz): Nieblum (Kertelheim), mehrfach!! und auf Amrum: beim Lesuchtturm, zwischen Xelsel und steenodde!! tauf den Inseln wohl angesäet).

Weber nennt das Gras als erster Florist des Gebiets (Prim. Fl. Hols. $8[1780])$.

\section{Formen.}

1. Rispenäste unterwärts ährchenlos, verlängert:

f. typica Hackel Monogr. Festuc. 150 (1882)。 - Häufigste Form. Dazil als Unterform:

f. subspicate A. u. Gr. Syn. Mitt.-Europ. Fl. II. 1. 503 (1900).

- Rispe sehr schmal; Rispenäste ein- bis zweiährig, ihre grundständigen Zweige einährig. - So zerstrent.

- Rispenäste fast vom Grunde mit Ährchen, kurz:

f. fasciculata Sonder Fl. Hamb. 64 (185̃1). - Selten. Hamburg: am Stadtoraben und am Elbufer (Sonder)!!, Steilshop und Gr. Borstel!!. Kiel (A. Christiansen!)。

\section{Mißbildungen.}

f. m. ramiflora nov. f. -- Pflanze aus den oberen Konoten mit verlängerten. rispentragenden Zweigen. - Hamburg: Besenhorster Elbrorland!..

138. (.ip). 58). Festuca arundinacea Schreber spic. Fl. Lips. 57 (1771). 4. Grundachse lockerrasig, mit bis $10 \mathrm{~cm}$ langen Ausläufern. Stengel aufrecht oder aufsteigend, $(0,4-10,6-1,1(-1.8) \mathrm{m}$ hoch, oberwärts blattlos, glatt oder ofter unter der Spitze rauh. Blattscheiden glatt oder an den unteren Blättern hesonders oberwärts rauh. Blattfläche $3-8 \mathrm{~mm}$ breit, flach, selten eingerollt. meistens beiderseits (hesonders oberseits) rauh. Blatthäutchen fast fehlend. Rispe $10-201-35$ ) $\mathrm{cm}$ lang, mit schlaffen, oft ïlerhängenden, seltener starren, aufrechten Ästen. Cnterer Rispenast so lang oder länger als die halbe Rispe, mit in der Regel wenigstens acht Ährchen; grundständiger Zweig (wem vorhanden) wenigstens dreiährig, selten nur zweiährig. Ährchen $7-12(-15) \mathrm{mm}$ lang. mit vier bis acht (bis elf) Blüten. Hüllspelzen spitz, wenig ungleich, untere bis 4, obere lis $4.5 \mathrm{~mm}$ lang. Deckspelzen $5-7-10) \mathrm{mm}$ lang, undentlich fünfnervig, 
spitz, zuweilen kul\% (--3 mm) begrannt, kahl, kium hautrandig. Fruchtknoten kahl. Blüte Juni bis August, einzeln später.

Am Strande und in seiner Nähe (an Deichen, Wegrändern und auf Weiden) sowie im (iebiete der Elbe und ihrer Nebenflüsse, der 'Trave, der Eider sowie der söberen Seen häufig bis nirht selten, im übrigen Bimnenlande zerstrent. an der Ostsee häufig und oft in enormer Menge. an der Tordsee seltener und oft spärlich. wenig auf den Nordfriesischen Inseln, und zwar auf Föhr (Schiötz) zerstreut!!, Amrum: Norddorf!!, Sylt: Morsumkliff (hammkiaer)!!, Wresterland, zwischen Keitum und Ar(hsum!!, Röm: Kirkeby (Raunkiaer) und Helgoland: am Ostabhang (Hallier, Ascherson, auch als $f$. multiflora [Brody!]).

Thre erste Erwähnung findet die Art für unser Gebiet bei Hornemann (Oec. Plantel. 3. Udg. 1. 119 [1821]) als F. elatior.

\section{Formen.}

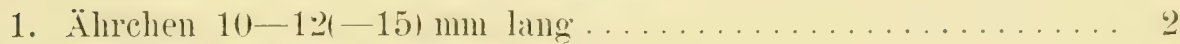

- Ährchen $7-9 \mathrm{~mm}$ lang . . . . . . . . . . . . . . . . . 4

2. Deckspelze gramnenlos (zuweilen einzclne ganz kurggannig) ... . 3

- Deckspelze mit 1-3 mm langer Granne:

f. orientalis Hackel Monogr. Festuc. 154 (1882). - Selten. Hamburg: am Elbufer bei Falkenthal (Zimpel 1892!). Glü ckstadt: Neuendeich!!. Neustadt: Grömitz!!. Kiel: Bülk! und Eckernförde: am Windebyer Moor! (A. Christiansen).

3. Pflanze $(0,3-) 0,5-1(-1,2) \mathrm{m}$ hoch; Blattfläche $3-6 \mathrm{~mm}$ breit; Rispe $10-20 \mathrm{~cm}$ lang; Ährchen bis $12 \mathrm{~mm}$ lang:

f. vulgaris Hackel a. a. O. 153 (1882). - Häufigste Form. Dazu zählen als Unterformen:

f. multiflora Sonder Fl. Hamb. 64 (1851). - Ährchen neun- bis elfblütig. - So auf fruchtbarem Boden, bisher: Hamburg (Sonder) mehrfach!!; Hadersleben: an der Föhrde (Christiansen!); Helgoland (Hallier).

f. fasciculata Sonder a. a. O. 64 (1851). - Rispe aufrecht, dicht, mit vom Grunde mit Ärrchen besetzten Ästen. - Hamburg (Sonder) mehrfach!!; Apenrade!!.

f. decolorans A. u. Gr. Syn. Mitt.-Europ. Fl. II. 1. 506 (1900). - Ährchen grün, in sehr lockerer Rispe. Hin und wieder auftretende Schattenform.

- Pflanze 1,5-2 m hoch; Blattfläche 6-8 mm breit; Rispe bis $35 \mathrm{~cm}$ lang; Ährehen bis $15 \mathrm{~mm}$ lang: 
f. gigantea P. Junge Jahrb. Hamb. Wiss. Anst. XXII. 66 (1905). - Eine auffällige Abart, deren untere, stark rauhe Blattscheiden an var. aspera Mutel erinnern, mit welcher auch die rauhen Blattnerven gemeinsam sind. Doch trennen von dieser Form die wenig rauhen Rispenäste und die fast gleichen, schmalen Hüllspelzen. - Stormarn: auf Alsterwiesen bei Wellingsbüttel 1903!! (ob angesäet?).

4. Blätter flach; Rispe groß, mit schlaffen Ästen:

f. baltica A. u. Gr. a. a. O. II. 1. 507 (1900). - So an der Ostsee von Heiligenhafen!! bis Hadersleben (Prahl).

- Blätter schmal, eingerollt; Rispe klein, starr, mit aufrecht abstehenden, wenigährigen Ästen; unterster grundständiger Zweig mit ein bis zwei Ährehen:

f. pauciflora Hartman Handb. Scand. Fl. 2. Uppl, 31 (1832). Kümmerform sehr trockener Standorte. - Bisher nur Hamburg: Warwisch 1911!!

\section{Mißbildungen.}

f. m. vivipara nov. f. - Ährchen in Laubsprosse auswachsend. - Hamburg: mehrfach am Elbufer!! (schon J. A. Schmidt 1865!).

\section{$\times($ sp. $57 \times 58)$. Festuca pratensis $\times$ arundinacea $=\boldsymbol{F}$. intermedia}

Hackel Monogr. Festuc. 151 (1882).

4. Grundachse lockerrasig. Stengel $0,6-0,8 \mathrm{~m}$ hoch, glatt, aufsteigend. Blattscheiden glatt. Blattfläche $3-6 \mathrm{~mm}$ breit, unterseits glatt oder schwach rauh. Rispe $10-15 \mathrm{~cm}$ lang, etwas schlaff, locker oder ziemlich dicht, ihr unterster Ast ziemlich kurz, mit vier bis sieben Ährchen, sein grundständiger Zweig mit zwei bis drei Ährchen. Ährchen 9-12(-18) mm lang, vier- bis achtblütig. Hüllspelzen ziemlich ungleich, schmallanzettlich, untere bis $3,5 \mathrm{~mm}$, obere bis $5 \mathrm{~mm}$ lang. Deckspelzen bis $8 \mathrm{~mm}$ lang, oberwärts hautrandig, spitz, kahl. Blüte Juni und Juli.

Mit den Eltern selten. - Hamburg: am Elbdeich in Ochsenwärder 1904!!. Pinneberg: an einem Graben der Elbwiesen bei Wittenbergen 1908!!. Lübeck (nach Friedrich Fl. v. Lüb. 43 [1895] von Hausknecht genannt; Geogr. Ges. Thür. III. 288 [1885]). — Eine im Formenreichtum der Eltern schwer mit Sicherheit zu erkennende Kreuzung.

139. (sp. 59). Festuca gigantea Villars Hist. pl. Dauph. II. 110 (1787). 4. Grundachse lockerrasig. Stengel aufrecht, oberwärts oft bogig übergeneigt, $0,5-1,5 \mathrm{~m}$ hoch, glatt. Blattscheiden am Stengelgrunde rauh, 
oben glatt, fast ganz gesehlosien. Blattflärohe $15-15-10(-15)$ mm breit, am Rande stark laul, somst sehwath rauh bis fast glatt, dumkelgriun. Blatthäutrhen ku\%. stumpt, mit zwei seitlichen G̈hrehen. Rispe meistens erol.s. otwa 15-30)(-40) ("m lang, mit abstehenden bis überhängenden, sehr verlängerten Istru. J̈hrehen (s - $11-15 \mathrm{~mm}$ lang. (zwei- bis) fünfbis nembliitig. Höllspelzen splitz, untere 3-3,5 mm lang, einnervig, obere t-5 mm lang, dreinervig. breithantrandig. Ineckspelzen his 7 mm lang, schwach fünfuervig, mit bis $18 \mathrm{~mm}$ langer Grame, hantrandig, kahl. Fruchtknoten kahl. Blüte Juni bis August.

In Wäldern und Gebüschen im Osten häufig, im Westen mehr zerstreut (doch auch in der Marsch nicht fehlend); auf den Nordfriesischen Inseln nur auf Helgoland (Hallier. Dalla 'Torre) und Föhr: am Königsgarten in $1 \mathrm{yk} ! !$

Weber nemnt die Art als Bromme gigantere (Prim. Fl. Hols. 9 [1780]).

Vom Typus weichen ab:

\section{Formen.}

f. triflora Koch Syn. Fl. Germ. et Helv. ed. 2. 942 (1844). Pflanze 0,4-0,7 m hoch; Rispe kurz, klein; Ährchen (zwei- bis) dreiblütig. - So an trockenen, schattigen Orten nicht selten.

f. nemoralis A. u. Gr. Syn. Mitt.-Europ. Fl. II. 1. 511 (1900)。Ährchen in sehr lockerer Rispe, grün, klein, vier- bis sechsblütig. - So in Wäldern zerstreut.

\section{Mißbildungen.}

f. m. vivipara nov. f. - Ährchen in Laubsprosse auswachsend. Hamburg (Erichsen!). Pinneberg: am Elbufer bei Teufelsbrïck (J. A. Schmidt 1865!).

$\times(* 1) .57 \times 59)$. Festuca pratensis $\times$ gigantea $=$ F. Schlickumi (irantzow Fl. d. Uckerm. $340(1880)$.

4. Grundachse lockerrasig. Stengel aufrecht oder etwas aufsteigend, 0,7-1 m hoch, glatt. Blattscheiden glatt, kahl. Blattfliiche bis $11 \mathrm{~mm}$ breit, oberseits stärker, unterseits schwächer rauh. Blatthäutchen fehlend. Rispe bis $25 \mathrm{~cm}$ limg, mit nur bis $8 \mathrm{~cm}$ lingen unteren Ästen, nach dem Verblühen zusammengezogen, schmal. Ährehen (8--) 10-15 mm lang. meistens fünf- bis achtblütig, mit oft schwach violett überlaufenen spelzen. Hüllspelzen ungleich. spitz, untere etwa 3,5 , obere etwa $5 \mathrm{~mm}$ lang, breit hautrandig. Deckspelzen lanzettlich, bis $7 \mathrm{~mm}$ lang, mit an den unteren meistens $4-6 \mathrm{~mm}$, zuweilen bis $10 \mathrm{~mm}$ langer Granne, die oberen oft fast grannenlos. Frucht fehlschlagend. 
Mit den Eltern selten.

Kiel: Dietrichsdorf 1910! und Marutendorf 1911! (A. Christiansen.

140. (.1). 60)1. Festuca silvatica Villar's Hist. 1)l. Dauph. II. 105 (1787).

7. Grundachse dichtrasig. Stengel aufrecht, $0,5-1,2 \mathrm{~m}$ hoch, glatt. Blattscheiden am stengelgrunde bleichbrämlich. etwas derb, glatt. der stengelliätter grün. eng. lauh l,is glatt. Blattläche der unteren Grumbliatter sehr hurz. fast frhlend. der folgenden Grundblätter sehr (-0.5 mı verlängert. dex stengedblätter hürzer. muterseits glatt, oberseits

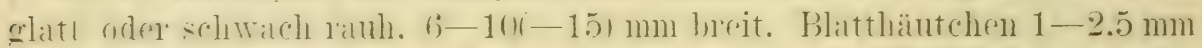
lang. Heit zerrisisn. Rispe 10-15 cm lang. lorker. mit geschlängelten Ästen. Älırchen 6-7 mm lang, (zwrei-bis) drei- bis fünfblütig. Hüll-

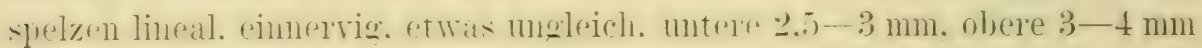
lang. Deckspelzen his 5.5) mun lang. schmal. spitz. gramnenlos. schwarh rauh. Fruchtknoten oberwärts behaart. Blüte Juni bis Juli.

In Lanlwäldern des istlichen fiebiets nicht seltens, südwärts weniger

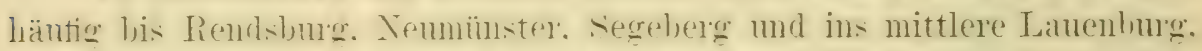

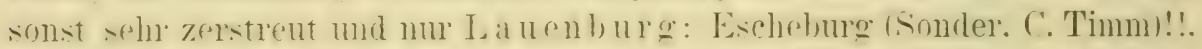

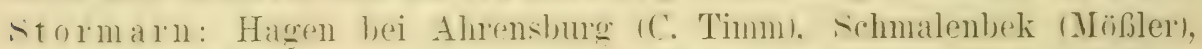

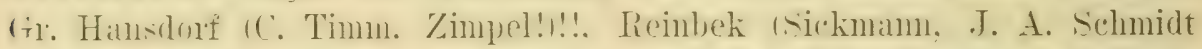

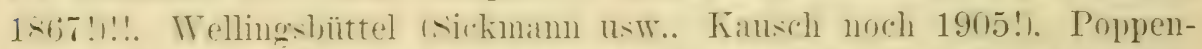

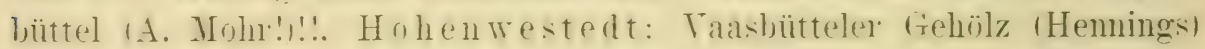
und Hadersleben: Gramm (Prahl) mehrfach!!.

Die erste Florennotiz hat für unser Gebiet Mrößler (Handb. Gewächsk. 135 [1815]).

Möglicherweise zählt Agrostis silvatica Weber (Prim. Fl. Hols. 7 [1780] hierher; doch ist keine Sicherheit darüber zu gewinnen. Im Kopenhagener Herbar liegt ein Exemplar, gesammelt "Ved Altona", „leg. Winthem"!; in neuerer Zeit ist das Gras dort nicht beobachtet worden. Sehr unsicher ist trotz des Belegexemplars auch Itzehoe als Standort (J. J.-Neyer 1817!) ; rgl. Bemerkung zu Koeleria glauca (p. 220).

\section{Formen.}

Vom Typ differiert wenig:

f. decidua Smith Engl. Bot. t. 2266 (1811). - Blätter schmal; Ährchen zwei- bis dreiblütig. - Ha mburg (Sonder Fl. Hamb. $6 \pm$ ohne weiteren Standort).

141. (adr. 72). Festuca rigida Kunth Enumeratio I. 392 (1833).

¿. rtengel zn mehreren. seltener einzeln. aus knickigem Grunde auficteigend, oft unterwärts verzweigt, glatt. Blattscheiden glatt, ober- 
wärts offen. Blattflïche $1-2 \mathrm{~mm}$ breit, unterseits meistens glatt, ober-

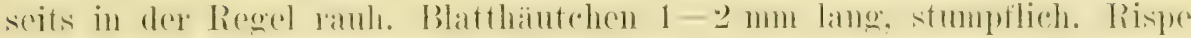

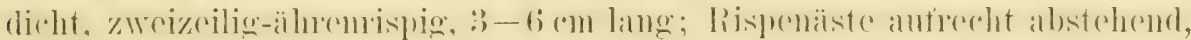
vom Grunde mit Ährchen. Ährchen 4-6 mm lang, vier- bis acht- (bis

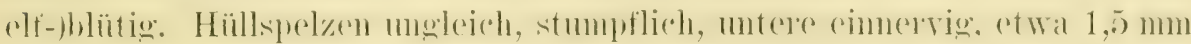
lang, obere dreinervig, etwa $2 \mathrm{~mm}$ lang. Deckspelzen 2-2,5 mm lamg, stmmpt, grammenlos. undentlieh fïnfuerrig. Blüte Juni bis Angust.

Auf Schutt bei Hamburg selten

Hamburg' (C. 'Timm!): Wollkämmerei am Reiherstieg' (J. Schmidt

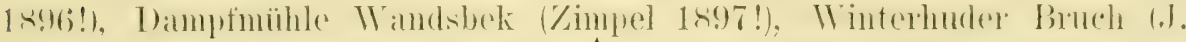
Schmidt 1890!, Dentsche Bot. Ges. X. 87 [1891]), Lokstedt (Laban $1889 !)$

142. (ady. 73). Festuca myuros [.. spee. plant. ed. 1. $7+11753)$.

$\odot$. Stengel zu mehreren, aufrecht oder aufsteigend, unterwärts oft verzweigt, $0,1-0,3 \mathrm{~m}$ hoch, glatt, bis zur hispe von Blattscheiden umweloen. Blattscheiden olatt. Blattflache $1-2 \mathrm{~mm}$ breit, oft ringerollt, glatt. Blatthäutchen fehlend. Rispe (j)-110-20 cm lang, sehr schmal, mit anflecht anliegenden Isten; unterster Ast viel kürzer als die halbe Rispe. Ährehen 9-10 mm lang, vier- bis tünfblütig. Hüllspelzen lineal, spitz, sehr ungleich, untere $(1-) 1,5-2 \mathrm{~mm}$, obere $4-5 \mathrm{~mm}$ lang. Deckspelzen bis 6 mm lang, schmal linzettlich, undeutlich fïnfnervig. an Rücken ramh, mit doppelt überragender, bis $14 \mathrm{~mm}$ langer Gramme. Fruchthuoten kahl. Blïte Juni bis August, zuweilen später.

Auf Schutt und an Wegrändern selten verschleppt.

Lauenburg: zwischen Billenkamp und Wohltorf (Laban 1873!, 1s90!). Hscheburg (Zimpel 1898!). H a mburg: Wollkimmerei am Reiherstieg (/impel 1894!, 1895!, Мohr 1906!), Georgswärder ('/mimpel 1894!). Ifammerbrook (.T. A. Schmidt mol Laban 1868!), bei der Dampefmühle Wandsbek (.J. S'chmidt 1894!, A. Mohr 1904!), Ejpendort (Laban 18!00!). am Diebsteich in Altona (C. 'Timm, Kausch 1885!). Kiel: Gaarden (Hennings 1877!). Hadersleben: Erleff (Prahl 1872!, 1877!); vgl. Bot. Ver. Brandenb. XIV. 147 [1872]).

Die älteren Standorte der Floren unseres Gebiets gehören nicht zu $F$. myuros. Bei Weber wird das Gras ohne weitere Bemerkung aufgeführt; wenn auch bei ihm F. dertonensis nicht fehlt (Prim. Fl. Hols. 8 [1780]), so dürfte seine Angabe sich doch auf diese Art beziehen, wie das bei Sickmann (Enumeratio 11 [1836]) und Hübener der Fall ist. Des ersteren Irrtum berichtigte schon Sonder (Fl. Hamb. 61 [1851]), des letzteren Belege im Kieler Herbar von 1822 (oder 1832?) sind $F$. dertonensis!. Auf diese Art bezielnt sich auch der Standort Süderbrarupholm bei Knuth (Fl. v. Schlesw.-Holst. 774 [1887]) (Nolte, Hb. Kiel!, Hb. Lübeck!). Bei Apenrade fand Bargum nur $F$. dertonensis (1799!). 
Tom Typus weicht ab:

\section{Formen.}

f. subunighumis A. 11. Gr. Syn. Mitt.-Europ. Fl. II. 1. 557 (1900). Untere Hüllspelze nur bis $1 \mathrm{~mm}$ lang. - Hamburg: Dampfmühle. Wandsbek (Zimpel 1894!).

143. (qs1). 10). Festuca dertonensis A. и. (ir. Sin. M[itt.-Emrop. Fl. II. 1. 559 (1900).

$\odot$. Stengel zu mehreren bis vielen, aufsteigend oder aufrecht, $(3-)$ 8-30 con hoch. glatt, zuweilen moterwärts rerzweigt. oberwärts blattlos. Blattscheiden glatt. oberwärts offen. Blattfläche $1-1.5 \mathrm{~mm}$ breit, eingerollt. glatt. Blatthäutchen fehlend. Risue $11-1.5-10 \mathrm{~cm}$ lang. schmal. mit aufrechten Ästen: unterster Rispeniast hath so ling wie die Rispe. Ährchen s-10 mm lang. vier- bis sechsblütig. Hüllspelzen lineal, spit\%. ungleich, untere $3,5-4,5 \mathrm{~mm}$, obere $5-6 \mathrm{~mm}$ lang. Deckspelzen bis 5.5 mm lang. schmallanzettlich. an Rücken glatt oder nur am Grannengrunde borstlich rauh; Grame etwa doppelt so lang wie ihre spelze. bis $11 \mathrm{~mm}$ ling. Fruchthnoten kahl. Blüte Mai bis Juli, einzeln später.

Auf und an Tregen. auf Brachäckern und samblfeldern, in Kiesgruben mon an sandigen Abhingen im sïdlichen Teile des Gebiets meistens nicht selten. doch bei $\mathrm{L}$ übeck nileht festgestellt und im nordöstlichen Holstein selten bei Yeustadt (Nolte 1822)!): Hospitahnïhle (Rohweder\%. Lütjenburg (Borchmann!) und Oldenburg (Borchmann!: am Fehmarusund (Lienan in Hb. J. A. schmidt!): weiter nördlich zerstrent. in Yordschleswigg selten und hier nur anf $A l s e n$ : Meelsfeld (H. Petersen). hei A penrade (Bargum 1799!), bei Hadersleben: Erleff (Prahlı!), am sliepsee (r. FischerBenzon und steinvorth) und bei Tondern: Kloyeng (H. Schmidt!); anf den Nordfriesischen Inseln nur anf Föhr (schiotz. Kertelheim 1896!) und Sylt (Hb. Kiel olne Finder!): Keitum (Bargum. Hb. Kiel!)!!, zwischen Ilorsm und Keitum (Taupell, Hb. Kopenhagen!), Westerland (Buchenau)!!. Braderup) (J. Schmidt 1!)(6!). Tinnum, Gr. und Ki. Mor'sum und Osterende!!.

Hierher F. bromoides bei Weber (Prim. Fl. Hols. 8 [1780]). Durch ein Versehen steht an Stelle des Namens $F$. dertonensis $(=F$. sciuroides Roth) in meiner Flora von Hamburg usw. 60 (1909) F. Danthonii.

Vom Typus weichen ab:

\section{Formen.}

f. gracitis A. u. Gr. Syn. Mitt.-Europ. Fl. II. 1. 559 (1901). Pflanze niedrig; Stengel einzeln, 3-10 $\mathrm{cm}$ hoch, mit einem bis sechs Ährchen. - Auf trockenem Boden zerstreut, z. B. La nen burg: Mrustin und Dermin!!, Kiel: Schulenhof, Elmschenhagen, Iönkeberg, Gaarden (A. Christiansen!), Sylt: Keitum!!. 
f. Broteri A. u. Gr. a. a. O. II. 1.559 (1901), - Pflanze bis $0,7 \mathrm{~m}$ hoch; Rispe bis $30 \mathrm{~cm}$ lang, am Grunde öfter unterbrochen; Ährchen 4-6 mm lang, drei- bis fünfblütig; Grannen zweibis dreimal so lang wie ihre Spelzen. - Hamburg: bei der Wollkämmerei am Reiherstieg (J. Schmidt 1894!).

Graphephorum arundinaceum Ascherson I'l. Brandenb. I. 852 (1864) fehlt im schleswig-holsteinischen Florengebiete. Die früheren irrtümlichen Angaben gehen auf Nolte zurück, der das Gras aufzählt (Nov. Fl. Hols. 12 [1826]). Eine Reihe späterer Floristen hat den Standort Noltes übernommen, z. T'. mit der Angabe Friedrichsort statt Friedrichstadt. F. v. Müller nannte als erster "Steinschleuse bei Süderstapel" (Breviar. Plant. 478 [1853]); ilm folgte anscheinend J. Lange (Flora Danica t. 2824 [1871]): „In flumine Eidora ad Süderstapel." Die Pflanze, gesammelt bei Friedrichstadt 1823, ist Festuca amudinacea Schreber! (nicht Liljeblad). Eine später nicht wiedererwähnte Notiz steht bei Hornemann (Vid. Selsk. phys. Skrift. I. Deel. I. Haefte 194 [1821]) als Arundo festreacea (nach Willdenow Enumeratio I. 126 [1809]), wo die Art aus Lauenburg, aber nicht aus Holstein, Erwähnung findet. Auch diese Angabe dürfte Nolte veranlaßt haben. Hornemann hat in seinen späteren Arbeiten $G$. arundinaceum nicht wieder genamnt, also wohl den Irrtum erkannt (rgl. Prahl Krit. Fl. 260 [1590]).

49. Gattung.

\section{Cynosurus.}

L. Gen. plant. ed. 5. $33(1754)$ z. T.

1. Spelzen der unfruchtbaren Ährchen spitz, unbegramnt .............. cristatus.

- Spelzen der unfruchtbaren Ährchen lang begrannt ............. C. echinatus.

144. (s1. 61). Cynosurus cristatus L. Spee. plant. ed. 1. 72 (1753).

4. Grundachse dichtrasig. Stengel meistens aufrecht, starr, $(0,1-)$ 0.2-0.5 m hoch, glatt. Blattscheiden glatt. geschlossen, die oberen schwach erweitert. Blattfläche 2-3 mm breit, oberwärts und am Rande rauh. Blatthäutchen etwa $1 \mathrm{~mm}$ lang. breit abgeschnitten. Rispe ährenfürmig. meistens :3-6 em lang und $6-8 \mathrm{~mm}$ breit, mit abwechselnden seitlichen Lappen. Ährchen in zwei Reihen. einseitig gestellt. jedes firuchtbare Ährehen ron einem unfuchtbaren begleitet. Unfruchtbares Ihrehen mit bis zehn linealen, spitzen. bis 3 mm lingen. gleichartigen spelzen. Fruchtbares Ährchen $13-14 \mathrm{~mm}$ ling. mit eimnervigen. spitzen. 2.5 und $3 \mathrm{~mm}$ langen. hautrandigen Hüllspelzen und bis :3 mm langen. lanzettlichen. undentlich fünfnerrigen. spitzen oder bis 1 mm ling begramnten. oberwärts oft rauhen Deckspelzen. Blïte Juni und Juli, zuweilen später.

Auf trockenen Wiesen und Tritten. an Abhängen und Teerändern häufig, auch auf den Nordseeinseln.

Zuerst genannt von Weber (Prim. Fl. Hols. S [1780]). 


\section{Formen.}

Tom Typus weicht als Kümmerform ab:

f. ovatus A. 11. Gr. Syn. Witt.-Europ. Fl. II. 1. ว69 (1900). Stengel nur 0,1 m hoch; Rispe kugelig bis eiförmig. - An trockenen Orten zerstrent, auch z. B. auf Sylt.

\section{Mißbildungen.}

f. "w. ricipars Willk. in Willk. und Lange Prodr. Fl. Hisp. I. 89 (1861). - Unfruchtbare Ährchen in Laubsprosse auswachsend. - Preetz!, Kiel: Teimer'sdorf!. Husum: Immenstedter Holz! 1.A. Christiansen). f. m. furcatus nov. f. - Rispe oberwärts oder bis zum Grunde gabelig

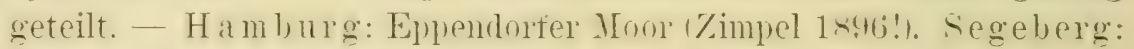
Westerrade (J. Schmidt 1892!).

145. ladv. 74). Cynosurus echinatus L. Nirec. plinlt. ed. 1. T2 (1753).

$\odot$. Stengel meistens zu mehreren, aufrecht oder aufsteigend, oft unterwärts verzweigt. 0,2-0.6 m hoch. unter der Rispe schwach rückwärts rauh. Blattscheiden grlatt oder schwach rauh. die oberen dentlich erweitert. Blattläche 2-61-111 mm breit. oberseits stäror. unterseits schwächer rauh. Blatthäutchen :3-5rissen. Rispe eiförmig-kopfig, (1-) $2-4 \mathrm{~cm}$ lang, bis über $2 \mathrm{~cm}$ breit. Fruchtbare Ährchen 6-i mm lang. drei- l,is rierblütig. ihre Hüllspelzen häutig. einnervig. wenig moleich. $5-6 \mathrm{~mm}$ und 6-6.5 mm lang, ihre Deckspelzen bis 5 mm lanқ. grün. seln undentlich nervig. oberwärts ranh. borstlich. bis $8 \mathrm{~mm}$ lang begrannt. Enfruchtbare Ährchen bis 7 mm lang, mit bis zाiölf linealen. in eine feine tramne ansgezogenen spelzen. Blïte Juni bis August.

Auf Schutt und unter Saat selten.

Ha mburg: bei der Wandsbeker Dampimühle tZimpel 1894!-1897!, Mohr 1906!), im Winterhuder Bruch (.Taap 1890!). am Diebsteich in Altona (Laban 1885!), bei der Rolindsmühle in Ottensen IC. Timm!: Laban in Fl. v. Holst. etc. 2022 [18ti6]). Ho henwestedt unter Getreide (Hennings 1873, Hb. Kiel!).

\section{อ̃o. Gattung.}

\section{Bromus.}

L. Gen. plant. ed. 5. 33 (1754) z. T.

1. Untere Hüllspelze drei- oder mehrnerrig, obere fünf- oder mehrnerrig ...... 2 .

- Vntere Hüllspelze ein-, obere dreinerrig .................... 12

2. Torspelze wenigstenc an den unteren Blüten les Ährchens so lang wie die

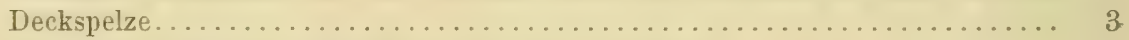

- Vorspelze an allen Blüten kürzer als die Deckspelze............... 5 
3. Deckspelzenränder mit stumpfem Winkel; Staubbeutel 2-3 mm lang.......

- Deckspelzenründer bogig verlaufend; Staubbeutel etwa $4 \mathrm{~mm}$ lang.

B. arvensis.

4. Deckspelzen sich nach der Blüte um die Frucht und Vorspelze zusanmenrollend, die Blïten daher durch Zwischemrïune getrennt

B. secalimes.

- Deckspelzen mit den Ründern auch nach der Blüte sich deckend; Granne stets

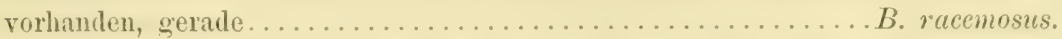

5. Deckspelzenränder bogig (zuweilen undeutlich winklig; dam Spelze grannenlos oder bis $2 \mathrm{~mm}$ lang' stachelspitzig)

- Deckspelzenränder deutlich stumpfwinklig.

6. Deckspelzen unbegramnt oder bis $2 \mathrm{~mm}$ lang stachelspitzig..............

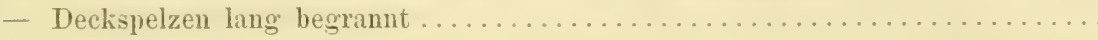

7. Ahrchen rundlich; Deckspelzen nicht gekielt .............. briziformis.

- Ärchen sehr stark zusammengedrückt; Deckspelzen scharf gekielt.

B. uniolioides.

8. Längste Deckspelzen bis $12 \mathrm{~mm}$ lang, im oberen Drittel die Granne tragend. B. macrostachys.

- Längste Deckspelzen bis $8 \mathrm{~mm}$ lang, im oberen Fünftel die Granne tragend;

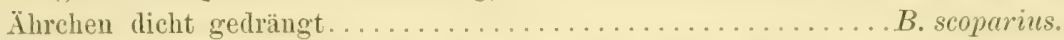

9. Staubbeutel etwa doppelt so lang wie breit, bis $1,5 \mathrm{~mm} \operatorname{lang} \ldots \ldots \ldots \ldots$.

- Staubbeutel wenigstens dreimal so lang wie breit, etwa $2 \mathrm{~mm}$ lang.........

10. Deckspelzen 4-9 mm lang, mit gleichartigen Grannen......... B. hordeaceus.

- Deckspelzen bis $10 \mathrm{~mm}$ lang, die unteren mit 3-5 mm langer, gerader, die oberen im ährchen mit bis $11 \mathrm{~mm}$ langer, auswärts gekrümmter Granme.

B. squarrosus.

11. Deckspelzen breitlanzettlich, mit an den oberen (odler allen) auswärts ge-

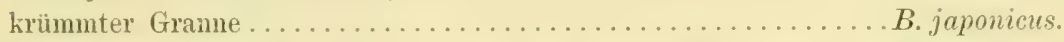

- Derkspelzen schmal rerkehrt-eiförmig, mit gerader Granne....B. commutatus.

12. Granne fehlend oder höchstens halb so lang wie die Deckspelze.......... 1

- Gramne wenigstens von halber Deckspelzenlänge.................. 14

13. Pflanze dichtrasig; Deckspelze bis $6 \mathrm{~mm}$ lang begrannt .......... B. erectus.

- Pflanze lockerrasig, mit kriechender Grundachse; Deckspelzen meist unbe-

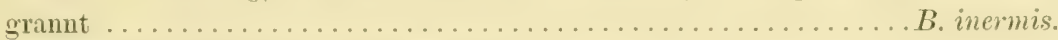

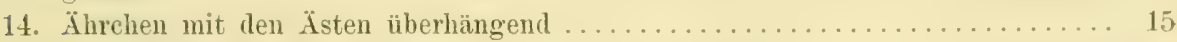

- Ährchen mit den İsten aufrecht ........................... 17

15. Pflanze 4; Ährchen gegen die Spitze verschmälert............ B. vamosus.

- Pflanze $\odot ;$ Ährehen gegen lie Spitze verbreitert.................. 16

16. Deckspelzen 10-12 mm lang, undeutlich nerrig, in der Regel kurzhanig.

B. tectorum.

- Dechspelzen 18-20 mm lang, deutlich nervig, rauh ............ sterilis.

17. Ährchenachse behaart; Deckspelzen mit deutlichen Nerren........ B. villosus.

- Alhrchenachse rauh; Deckspelze mit undeutlichen Nerven...... B. madritensis.

146. (1). 62). Bromus ramosus Hudson Fl. Angl. ofl. 1. 40 (176:) erw. 7. Grundachse kriechend. Stengel aufrecht, $(0,5-) 0,6-1,2(-2) \mathrm{m}$ hoch. dicht mit rückwiutsersellten Härehen besetzt. Blattseheiden ene. lauhharrig mit $2-3 \mathrm{~mm}$ langen Haren oder die oberen kurzharig mit nicht 1 mm langen Härehe'n. Blattfläche 6-12(-15)) mm breit, unterseits glatt und rauhhaarig, oberseits und am liande ramh. Blatthäutchen 
bis $3 \mathrm{~mm}$ lang, stumpf. Rispe 10-40 cm lang, breit oder schmal, mit verlïngerten Ästen und entferntgestellten Ährchen. Ährchen $2-3 \mathrm{~cm}$ lang, vier- bis sieben- (bis nem-)blütig. Hüllspelzen spitz, sehr ungleich, untere lineal, eimnervig, etwa 5-6 mm lang, obere lamzettlich, dreinervig, 9-10(-13) mm lang. Deckspelzen 10-12(-16) mm lang, schmallanzettlich, dreinervig, unterwärts am Rücken oft behart. schmal hautrandig, undeutlich zweizähnig. mit 8-10 mm langer Gramme. Blüte Juni bis August.

In schattigen, weniger in lichten. Laubwäldern im Osten nicht selten (südwärts weniger verbreitet), im Westen selten.

Zu dieser Art gehört B. altissimus Weber Prim. Fl. Hols. 9 Nr. 94 (1780).

\section{Formen.}

Die Art zerfällt in zwei Rassen:

var. eutramosus A. u. Gr. Syn. Mitt.-Europ. Fl. II. 1. 575 (1900). - Stengel $(0,8-) 1-1,5(-2) \mathrm{m}$ hoch; Blattscheiden sämtlich rauhhaarig; Rispe $0,2-0,4 \mathrm{~m}$ lang, mit bis über $20 \mathrm{~cm}$ langen unteren Rispenästen; Rispenäste weit abstehend, mit einem grundständigen Zweige; das verkümmerte, am Stengel etwas herablaufende Tragblatt des untersten Astes zuweilen am Rande rauhhaarig.

So häufiger, doch um Hamburg nur zerstrent, im Westen nur bei Hadersleben: Gramm (J. Schmidt!).

var. Benekeni A. u. Gr. a. а. O. II. 1. 576 (1900), -- Stengel 0,5-1 m hoch; obere Blattscheiden dicht weich kurzhaarig; Rispe 0,1—0,2 m lang, mit kürzeren, wenig abstehenden Ästen, daher schmal; untere Rispenäste mit (1-)2-5 grundständigen Ästen, mit kahlem, nicht herablaufendem Tragblatt.

So seltener. - Im Osten sehr zerstreut, südwärts bis Eutin: Ugleisee (Krause), Lübeck: Timmendorfer Wohld (Ranke 1893!), Lauerholz (Friedrich 1882!) und Stormarn: Graskoppel und Haidkamper Wohld bei Zarpen (Rohweder!), Forst Reinfeld!!, im Westen nur bei Husum: Immenstedter Holz (A. Christiansen!) und bei Hadersleben: Gramm!!.

Über das Verhalten beider Arten vgl. die erwähnte Synopsis II. 1. 576 (1900), ferner Lange Haandb. Iansk. Fl. 4. I'dg. 98/99) (1886) und Prahl (Krit. Fl. 261/262 [1890]).

147. (qsp). 11). Bromus erectus Hudson Fl. Angl. ed. 1. 49 (1762) subsp). eu-erectus A. 11. Gr. Syn. Mitt.-Europ. Fl. II. 1. 585 (1901).

4. Grundachse dichtrasig. Stengel aufrecht, $0,4-0,8(-1) \mathrm{m}$ hoch, glatt. Blattscheiden kahl oder an den unteren Blättern zerstreut kurzhaarig. Blattfläche der Grundblätter $1-3 \mathrm{~mm}$ breit, gefaltet, entfernt 
langhiarig gewimpert. der stengelblätter in der Regel flach, 2-41-5) mm breit, kahl oder unterseits kurhatrig. glatt. Blatthäutehem $1-2$ mm lang, breit zerrissen. Rispe schmal, aufrecht, (7-)10-20 cm lang, mit

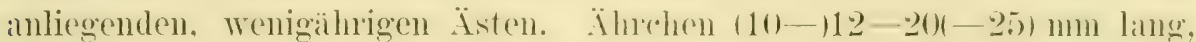
vier- bis sieben- (bis zeln-blüitig. Jïllspelzen spitz, mitere ainnervig (zuweilen mit zwei schwachen seitennervent, otwa!) mm lang, obere deeinervig. 10-11 mm lang. I)eckispelzen fünfnerrig. 10-12 mm lang, lanzett-

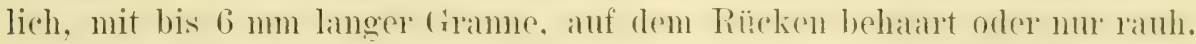

Auf Wiesen und Grasland, in und an Wegen, an Abhängen und Dimmen in ästlichen Holstein selne zerstreut, Westwärts und noldwärts

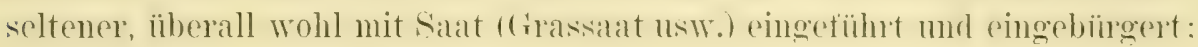
bei Hamburg auch auf Schutt.

La u en bur g: Gülzower Park (Klatt), Witzeeze!!, St. Georgsberg bei Ratzeburg 1912!!. Hamburg: zwischen Bergedorf und Rotenhaus (J. A. Schmidt 1873!), Hamm (Sonder!; im Hb. Lülb.: Horn!), vor dem I) ammor (Nonder, Kincke 18(b5!), Uhlenhorst mehrfach (Laban 1884!), Winterhude mehrfach (C. Timm 1885!, .J. Schmidt 1890!), Evpendorf (Iaban 1890!), Kil. Grasbrook (Zimpel 1897!). Stormaln: Poppenbüttel (.T. schmirlt 18.3 !). Pinneberg: Flottbek (Śonder), zwischen Mühlenbelg und Nienstedten (Laban 1867!)!!. L üb eck: am Bahnhof und Hafen (Friedrich 1894!), zwischen Blankensee mod Pogeez am Bahndamm (I'rihl 1R92!) und ron hier bis Gr. Saran (Friedrich 1893!). Segeberg: Waldweg im Forste Mönkloh 1911!.. L ütjenburg: ,.Lippe* bei Hohwacht 1912!!. Kiel: Bahndamm bei Neumühlen 1908!!, Nönkeberg!. Knoop! und Probsteierhagen! (A. Christiansen). Rendsburg: Dickenhörn (A. Christiansen!). Schleswig: Poeler Wiesen (Hinrichsen 1853!). Hadersleben: Fredstedt (Prahl 1873!). Amrum: beim Leuchtturm 1912!:. Helgoland: Festungsbaugelände im Oberland (Ascherson 1899) $1912 ! !$

Von den Floristen des Gebiets sammelte Nolte als erster B. erectus (Nov. Fl. Hols. 13 [1826]), aber nicht im Gebiete, sondern nahe der Grenze bei Mechow in Fürstentum Ratzeburg (daher bei Borchmann, Holst. Fl. 81 [1856] der Standort Ratzeburg). Weun Knuth (Fl. v. Schlesw.-Holst. 782 [1887]) Nolte als Gewährsmann für ein Auftreten dieser Trespe zwischen Blankenese und Neumühlen nennt, so dürfte irgendeine Verwechslung vorliegen (vielleicht mit $B$. inermis). Ebenfalls nicht sicher resp. unrichtig sind die Angaben des Auftretens zwischen Schiffbek und Schleems (Hübener Fl. r. Hamb. 503 [1847]) sowie bei Lütjenburg (Borchmann nach Knuth a. a. 0.). Wenn Knuth die Stampfmühle bei Schleswig als Ort des Vorkommens nemnt, so ist wohl Hinrichsens Fundort gemeint, den Lange (Haandb. Dansk. Fl. 3. Udg. 86 [1864]) als "Poek" bezeichnet.

\section{Formen.}

1. Alle oder die unteren Blattscheiden behaart: f. longiflor'ss Parlat. Fl. Ital. I. 413 (1848). - Untere Blätter flach. 
So selten. - H a mburg (Zimpel Dentsche Bot. Monatsschr. XTII. 125 [1899]): zwisehen Bergedorf und Rotenhaus (J. A. Schmidt 1873!). - Ubergangsformen mehrfach.

- Alle Blattscheiden kahl.

f. typicus A. u. Gr. a. a. O. II. 1. 586 (1901). - Grundblätter gefaltet.

Zerfällt in mehrere Formen; bei uns

f. glabriflorus Borbas Österr. Bot. Zeit. XXXII. 135 (1882).

- Deckspelzen kahl. - Häufigste Form, durch allmähliche Übergänge mit der folgenden verknüpft.

f. villosus Kunth Enumeratio I. 418 (1833). - Deckspeizen dicht behaart. - Hamburg: Winterhuder Alsterufer (C. Timm 1885!). Kiel: Neumühlen 1908!!.

f. depauperatus A. u. Gr. a. a. O. II. 1.586 (1901). - Unterster Rispenast ohne oder nur mit einem grundständigen Zweige. - So typisch im Gebiete nicht beobachtet, in Übergangsformen z. B. Hamburg: Winterhude (Jaap 1887!) und nahe der Grenze des Gebiets in Boizenburg!!.

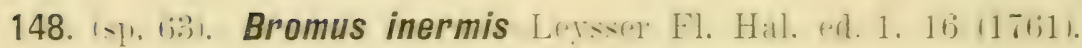

4. Grundachse weit kriechend, mit selır verlängerten Ausläufern.

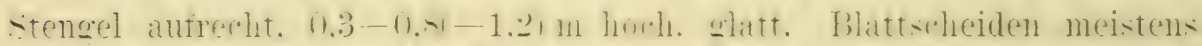

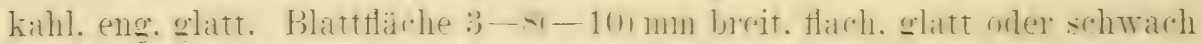
ranl. am Rande rath. Blatthäutchen fast fehlend. stumpt. selten lis

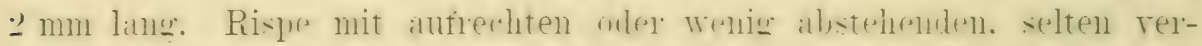

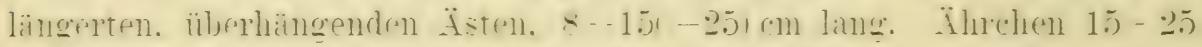

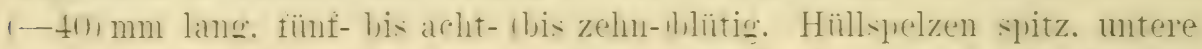

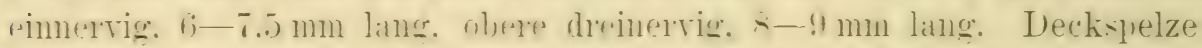

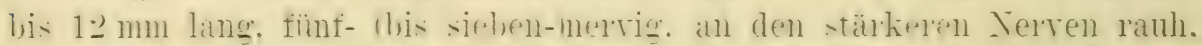
stumpflich, seltener mit hurzer, $1-3 \mathrm{~mm}$ langer Granne. Blïte (Mai) Juni bis .Juli.

Auf Tiesen und Grasland, an Abhängen und Deichen, zuweilen anch anf Schutt.

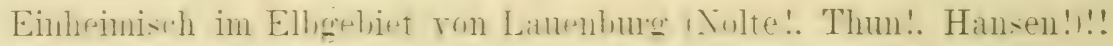

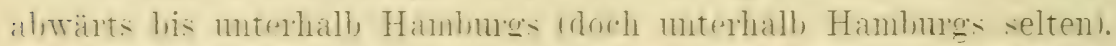

Im ïhrgen frebiete rerschleupt. zuweilen eingebürgert. Hamburg:

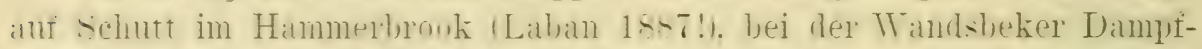

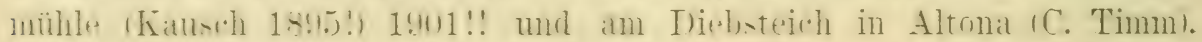

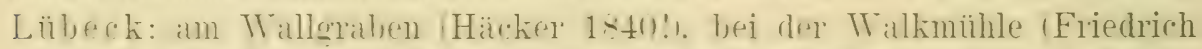
1-92). zwishen Burg- und Hüxtertor (T. Bmmeter 1!12!!. Fehmarn:

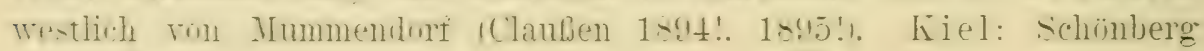


(J. T. Meyer 1820!), Voonde (('hristiansen 1912!). Rendshurg: Westerrönfeld (A. Christiansen 1912!). Flensburg: bei der Ziegelei an der Ballastbriicke (Hansen 1842!, Prahl 1876!, Gelert 1898!).

Bereits Weber zählt B. inermis mit dem Synonym Festuca speciosa (nach Schreber) auf (Prim. Fl. Hols. 8 [1780]). Der erste Hamburger Florist, der sie bringt, ist MIöBler; der Billwärder als Fundort nennt (Handb. Gewächsk. 141 [1815]). Nach der Flora Danica kommt $B$. inermis bei Schlutup vor (t. 1805 [1825]); es ist damit wohl der Standort auf dem Buchwerler gemeint (Nolte 1821!). Notizen bei Borchmamn (Fl. r. Holst. 80 [1856]) für den Heirlkrug, „Steinwarde" (Stemwarde) und Friedrichsruh, bei Lienau (Fl.v. Eutin 3 [1863]) für Eutin, bei Laban (Fl. v. Holst. 227 [1866]) für Grömitz und bei Hennings (Naturw. Ver. Schlesw.-Holst. II. 1. 205 [1876]) sind unrichtig oder ganz unsicher.

\section{Formen.}

1. Deckspelze unbegrannt .

- Deckspelze mit 2-3 $\mathrm{mm}$ langer Granne:

f. aristatus Schm Enum. plant. Transs. 805 (1866). - Zerstreut. - La uenburg: Lauenburg (J. A. Schmidt 1872!)!!, Geesthacht (Zimpel!)!!, Escheburg (J. A. Schmidt 1866!). H a mburg: Zollenspieker (Pieper!), Warwisch!!, MLoorwärder (J. Schmidt!). Lübeck: am Wallgraben (Häcker!). Flensburg: (Hansen!). - Mit der Form oder auch ohne sie treten Pflanzen auf, bei denen nur ein T'eil der Deckspelzen begrannt ist.

2. Blätter oder Deckspelzen behaart.................

- Blätter und Deckspelzen kahl:

f.typicus Beck Fl.Nied.-Oesterr. I. 106 (1890), -- Hänfigste Form.

Dazı zählen als Unterformen:

f. laxus P. Junge Naturw. Ver. Hamb. 3. F. XVII. 46 (1910). - Stengel 1-1,2 m hoch; Rispe überhängend, schlaff, mit schlaffen, oft stark verlängerten Ästen. Hamburg: Kirchwärder!!. Kiel: Voorde (A. Christiansen!).

f. latifolius nov. f. - Blätter 8-10 mm breit. - Lauenburg: Escheburg (J. A. Schmidt 1866!).

3. Blattscheiden und -flächen behaart; Deckspelzen kahl:

f. pellitus Beck a. a. O. I. 106 (1890), - Kiel: Voorde (A. Christiansen!).

- Blätter kahl; Deckspelzen dicht kurzhaarig:

f. villosus Beck a. a. O. I. 106 (1890). - La u enburg: in der Aue!!. - Eine Übergangsform bei Voorde!.

149. (sp. 64). Bromus sterilis L. Spec. plant. ed. 1. 77 (1753).

$\odot, \odot$. Stengel zu mehreren, aufrecht oder aufsteigend, $0,2-0,8 \mathrm{~m}$ 
hoch, glitt oder unter der Rispe schwach rauh. Blattscheiden eng, kurz weichlaarig, seltener rauhharig. Blattflärhe $2-5 \mathrm{~mm}$ breit, glatt, beiderseits kurz beharat bis fast kahl. Blatthäutchen (2-)3-4 mm lang, fein zerschlitzt. Rispe $10-20(-30) \mathrm{cm}$ lang, mit schlaffen, überhängenden, wenigährigen İsten. Ährchen $3-3,5 \mathrm{~cm}$ lang, vier-bis sieben- (bis elf-) blütig. Hüllspelzen lineal, spitz, sehr ungleich, untere einnervig, $9-10 \mathrm{~mm}$ lang. olsere dreinerrig, 15-16 mm lang. Deckspelzen $18--20 \mathrm{~mm}$ lang, deutlich hervortretend siebennervig, ranh, selten behaart, mit bis $3 \mathrm{~cm}$ langer Granne.

An Wegrändern, Hecken, Zämen, auf Wällen, seltener an Abhängen, im östlichen und mittleren Gebiet lı̈̈nfig oder nicht selten, doch nordwärts weniger allgemein verbreitet; im Westen nur bei Husum (F. r. Miüller); auf den Nordseeinseln nur auf Helgoland (Hoffmamn) früher (schon ron Hallier nicht mehr beobachtet) und auf Föhr: Wyk!!.

Erwähnt für das Gebiet Flora Danica t. 1325 (1808) und ron Nolte in den Novitien p. 13 (1826).

\section{Formen.}

Vom Typus weichen ab:

f. pubescens nov. f. - Deckspelze auf dem Rücken dicht behaart. - Hamburg: bei der Wandsbeker Dampfmühle 1902!!.

f. oligostachys A. u. Gr. Syn. Mitt.-Europ. Fl. II. 1. 592 (1901)。 Pflanze 5-12(-20) cm hoch, mit ein- bis dreiähriger Rispe. Kiel: Gaarden (A. Christiansen!).

f. lamuinosus Rohlena Böhm. Ges. Wiss. Ber. XXIV. 4. 7 (1899). - Untere Blätter an Scheide und Fläche abstehend lang behaart. - Anscheinend verbreitet.

150. (sp. 65). Bromus tectorum L. Spec. plant. ed. 1. 77 (1753).

$\odot$, öfter $\odot$. Stengel zu mehreren bis vielen, aufrecht oder aufsteigend, $(0,1-j 0,2-0,3(-0,6)$ m hoch, glatt oder unter den Kinoten fein kurzhaarig. Blattscheiden im untersten Teile oft kahl, sonst dicht kurzhaarig, oberwärts mit zerstrenten, langen Haaren. Blattfläche 2-4 mm breit, fein kurzharig, am Rande rauh und im unteren Teile oft gewimpert. Blatthäutchen 1-2 $\mathrm{mm}$ lang, fein haarig zerschlitzt. Rispe $5-15 \mathrm{~cm}$ lang, mit überhängenden, ziemlich kurzen Ästen. Ährchen 11-20 mm lang, vier- bis acht- (bis zwölf-)blütig. Hüllspelzen spitz, ungleich, untere 6-7 mm lang, eimnervig, obere 9-10 mm lang, dreinervig, beide am Kiele rauh. Deckspelzen 10-12 mm lang, undeutlich nervig, am Rücken behaart oder rauh, aus der zweizähnigen Spitze mit bis $16 \mathrm{~mm}$ langer Granne. Blüte Mai bis Juli, zuweilen bis November. 


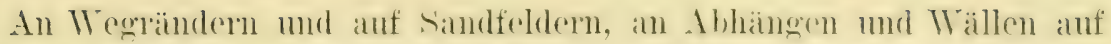
trockenem Boden im sïdöstlichen Gebiet bis Hamburg-Lïbeck-Thavemünde nicht selten bis zerstreut, besonders längs der Elloe. nordwärts besonders längs der Balndamme (und Kanäle) selne zerstrent bis fonderburg (H. Petersent, II adersleben: bei der sichiffsbrücke 1.1. Christiansen 1907!) und 'Tonder'n: Hoyer (Friderichsen 1898!).

Weber erwähnt B. tectorum (Prim. Fl. Hols. 9 [1780]); die Abbildung der Flora Danica (t. 1806 [1825]) wurde nach Nolteschen Pflanzen aus Lauenburg gezeichnet. Wolf notierte das Gras vor 1816 für Lübeck. Mehrere von Knuth (Fl. v. Schlesw.-Holst. 783 [1887]) auf(renommene Standortsangaben sind ron höchst zweifelhafter Art: Lütjenburg (Borchmam); Hohwacht (Hansen) unl: Lam Oldenhurg ziemlich häufig (Corlt). Es wird sich wohl um B. sterilis gehandelt haben, ebenso bei Lienau (FI. r. Eutin 3 [1863]): „Auf trockenen Anhöhen, z. B. am großen See hinter der Schäferei."

Herbstblühende Exemplare liegen in Herbar Zimpel von Hamburg: Dampfmühle Wandsbek (Oktoher 1892!, November 1894!). Etwas frülzeitiger fand sich die gleirhe Form in Lauenburg: Roseburg (15. September 1912!!).

Vom Typus weichen ab:

\section{Formen.}

f'. mudus M. u. K. Deutschl. Fl. I. 689 (1823)。 - Hüll- und Deckspelzen unbehaart. - Hamburg (Sonder als f. glabratus): Dampfmühle Wandsbek (W. Hansen 1893!), Diebsteich (Beyle 1885!), Bahrenfeld!!. Neumünster: Einfeld! und Kiel: Hof Krog! und Kronsburg! (A. Christiansen). - Außerhalb des Gebiets mehrfach am Elbufer!!.

f. longipilus Borbas Öst. Bot. Zeit. XXXII. 135 (1882)。 - Haare der Blätter, Äste und Ährchen verlängert. - Verbreitete, wenig wichtige Form.

151. (adv. 75). Bromus villosus Forsk. Descript. 39 (1765).

$\odot$. Stengel zu mehreren, aufrecht oder seltener aufsteigend, 0.3-0.7 m hoch, glatt, unter der Rispe zerstreut behaart, sonst kahl. Blattscheiden oberwärts dicht behaart. unterwärts oft kahl. Blattfläche beiderseits behaart, $2-5 \mathrm{~mm}$ breit. Blatthäutchen $2-4 \mathrm{~mm}$ lang, fein zerrissen. Rispe dicht oder locker, mit aufrechten Ästen, $7-15 \mathrm{~cm}$ lang. Ährchen meistens einzeln, etwa $3.5 \mathrm{~cm}$ lang, fünf- bis siebenblütig. Hüllspelzen ungleich, spitz, untere einnervig (zuweilen dreinerrig), $17-19 \mathrm{~mm}$ lang, obere dreinerrig, $23-28 \mathrm{~mm}$ lang. Deckspelzen bis $26 \mathrm{~mm}$ lang, deutlich zweizähnig. am Rücken kurz dicht behaart, mit stark rauher, bis $5,6 \mathrm{~cm}$ langer Granne. Blüte Nai und Juni.

Auf Schutt bei Hamburg selten.

Hamburg: Georgswärder (Zimpel 1894!. 1895!), Hammerbrook mehrfach (Bruns 1886!) (Prahl Krit. Fl. 262 [1890]). 
Formen.

Die Art zerfällt in zwei Rassen:

var. maximus A. u. Gr. Syn. Mitt.-Europ. Fl. II. 1. 595 (1901).

- Ährchenstiele 2-4,5 cm lang; daher Rispe locker; Granne

$3-5,6 \mathrm{~cm}$ lang, bis fast doppelt so lang wie ihre Spelze.

So am ersten Standorte!.

var. rigidus A. u. Gr. a. a. O. II. 1. 596 (1901). - Ährchenstiele

0,5-1 cm lang, daher. Rispe dichter; Granne 2-2,5 cm lang, etwa so lang wie die Deckspelze.

So am zweiten Standorte!. — Wem Laban (Fl. v. Hamb. 4. Aufl. 198 [1887]) Teufelsbrück als Fundort, Bruns als Finder nennt, so liegt eine Verwechslung vor.

152. (adv. 76). Bromus madritensis L. Amoen. acad. IV. 265 (1755).

$\odot$. Stengel zn mehreren, anfrecht oder öfter anfsteigend, kahl, glatt. Blattscheiden weit offen, untere rïckwaits behart, obere kahl. Blattfläche $1-3 \mathrm{~mm}$ breit, beiderseits kurzharig. Blatthäutchen $1-3 \mathrm{~mm}$ lang, fein zerschlitzt. Rispe $5-10 \mathrm{~cm}$ lang, mit aufrechten, kurzen, einbis vierährigen Asten. Threhen 2-2,5 cm lang, fünf- bis acht- (bis zwölf-) blütig. Hüllspelzen lineal, häutig, untere 7-10 mm lang, stets einnervig. obere 11-14 mm lang, dreinervig. Deckspelzen bis $19 \mathrm{~mm}$ lang, undentlich nervig, kurzhaarig, mit bis $2,7 \mathrm{~mm}$ langer Granne. Blüte Mai und Juni.

Auf Schutt bei Hamburg selten.

Hamburg: Steilshop (Herbst, Deutsche Bot. Ges. X. 86 [1892], J. Schmidt 1891 !).

Eine Pflanze von Eppendorf: Lehmweg (Laban 1890!, A. Junge Ver. Naturw. Unterh. VIII. 66 [1894]) ist B. sterilis.

153. (qsp. 12). Bromus secalinus L. Spec. plant. ed. 1. 77 (1753).

$\odot$ und $\odot$. Stengel einzeln oder zu wenigen, aufrecht oder aufsteigend, 0,2-1,2(-1,6) m hoch, glatt. Blattscheiden eng, kahl oder selten behaart. Blattfläche $2-6 \mathrm{~mm}$ breit, am Rande rauh, oberseits behaart, unterseits kahl. Blatthäutchen etwa $1 \mathrm{~mm}$ lang, breit, unregelmäßig zerrissen. Rispe $4-15(-20) \mathrm{cm}$ lang, mit aufrechten oder einseitig überhängenden Ästen; Äste verlängert, mit bis acht Ährchen und bis vier grundständigen Zweigen. Ährchen $(1-) 1,5-2,5 \mathrm{~cm}$ lang, mit $5-11(-15)$ Blüten. Hüllspelzen stumpf, untere dreinervig, 4,5 bis $5 \mathrm{~mm}$ lang, obere fünfnervig, 5,5-7 mm lang. Deckspelzen $(5-) 7-11 \mathrm{~mm}$ lang, kahl, selten behaart, stumpf, grannenlos oder mit bis $1 \mathrm{~cm}$ langer, 


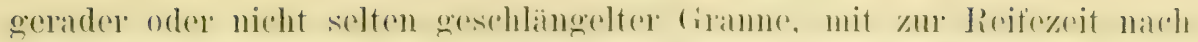

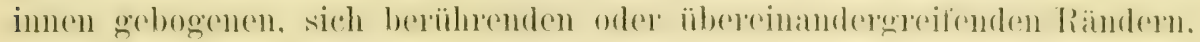
daher die Blïten getrennt. Torspelze so lang wie die Deckspelze. An-

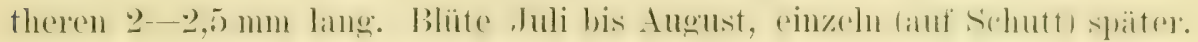

Auf Äckern, weniger an Wegen und anf Schntt, häufig bis nicht selten, auch auf den Nordfriesischen Inseln. Föhr: Boldixum (J. A.

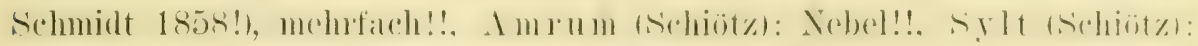

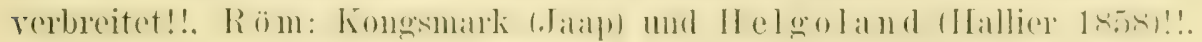

Zuerst erwähnt ron Weber (Prim. Fl. Hols. 8 [1780]).

\section{Formen.}

Die Art zerfällt in zwei Rassen:

1. var. multiflorus Ascherson Fl. Brandenb. I. 863 (1864). - Ährchen 2-2,5 cm lang, 12-15blütig, untere Hüllspelze etwa $8 \mathrm{~mm}$, obere etwa $9 \mathrm{~mm}$ lang; Deckspelzen bis $11 \mathrm{~mm}$ lang, mit bis $1 \mathrm{~cm}$ langer Gramne.

So bei Hamburg genannt, aber anscheinend umichtig, rerwechselt mit $f$. polyanthus.

- var. vulgaris Koch Syn. Deutsch. u. Schweiz. Fl. ed. 1. 819 (1837). - Ährchen 1,5-2 cm lang, selten länger oder kürzer, mit 5-8 (-14) Blüten, untere Hüllspelze etwa $5 \mathrm{~mm}$, obere etwa $7 \mathrm{~mm}$ lang. Deckspelzen nur bis $8 \mathrm{~mm}$ lang, mit kurzer oder bis $9 \mathrm{~mm}$ langer Granne oder grannenlos.

Gliedert sich in mehrere Formen:

f. glabratus A. 1. Gr. Syn. Mitt.-Europ. Fl. II. 1. 604 (1901). Blattscheiden kahl; Spelzen kahl; Gramne fehlend oder wenige Millimeter lang und stark geschlängoelt. — Häufigste Form.

f. hirtus A. u. Gr. a. a. O. II. 1. 604 (1901). - Blattscheiden kahl; Spelzen behart; Granne kurz oder fehlend. - Selten. Stormarn: Steinbek (Sonder). Hamburg: Dampfmïhle Wandsbek (Zimpel 1894!, z. 'I'. einährige, niedrige Pflanzen [f. monostachys mh.]).

f. polyanthus Beck Fl. Nied.-Oesterr. I. 108.(1890). - Ährchen 12-14blütig; sonst mit den Merkmalen der beiden rorhergehenden oder der beiden folgenden Formen. - Zerstreut. H a m burg: Elbinseln (Sonder als f. grossus), Kuhwärder (C. 'Timm 1881!), Steinwärder und Kl. Grasbrook (J. A. Schmidt!). Hammerbrook (Klatt!, Jaap 1892!), Wandsbek (J. A. Schmidt 1869 !), Winterhude (Zimpel 1892!), Eppendorf (Laban 1890!), Harvestehude (Zimpel 1890!), bei der Holstenbramerei Altona (Zimpel 1892!), Bahrenfeld 1900!!, Teufelsbrïck (Sonder Hb. Lübeck als B. commutatıs!), Langenfelde (H. Röper!). Lauen- 
b urg: Stintenburger Hütte!!. Segeberg: Hamfelde (Pieper!). Flensburg (Hb. J. J. Meyer!). Husum: Ahrenviöl (A. Christiansen!).

f. elongatus A.u. Gr.a.a. O.II. 1. 604 (1901). - Granne 6-9 mm lang, gerade. Sonst mit den Merkmalen der drei vorgenannten Formen. - Zerstreut, oft spärlich.

f. lasiophyllus Beck a. a. O. I. 108 (1890), - Blätter (auch die Scheiden) behaart. - Husum: Ahrenviöl (A. Christiansen!).

Zwei in der Literatur für unser Gebiet erwähnte Formen: $f$. velutinus (Schrader) Koch und $f$. hordeaceus "Gmel." (Gmelin als Art) kommen nicht vor; in beiden Fällen hat es sich (sicher oder wahrscheinlich) um $f$. hivtus A. u. Gr. gehandelt (bei Knuth, Fl. v. Schlesw.-Holst. 780 [1887]).

154. (qsp. 13). Bromus arvensis L. Spec. plant. ed. 1.77 (1753).

$\odot$ oder öfter $\odot$. Stengel zu mehreren bis vielen, meistens aufrecht, 0,3-1 m hoch, glatt. Blattscheiden dicht kurzhaarig. Blattfläche $2-\tilde{5}$ (-8) $\mathrm{mm}$ breit, an Rande rauh, beiderseits behaart. Blatthäutchen $1-2 \mathrm{~mm}$ lang, zerschlitzt. Rispe $8-20(-30) \mathrm{cm}$ lang, mit gestreckten, dümnen, bis $15 \mathrm{~cm}$ langen Ästen, die mehrere grundständige Zweige besitzen. Ährchen in der Regel 1,5-2(-2,5) cm lang, mit (5-)7-11(-15) Blüten. Hüllspelzen etwa 5 und $6-7 \mathrm{~mm}$ lang, spitz. Deckspelzen meistens $5-7,5$, selten bis $10 \mathrm{~mm}$ lang, rauh, mit bis $9 \mathrm{~mm}$ langer Granne. Vorspelze so lang wie die Deckspelze. Antheren etwa $4 \mathrm{~mm}$ lang; rielmal länger als breit. Blüte Juni und Juli, zuweilen bis Oktober.

Auf Äckern, an Feldrainen und Wegrändern, auf Schutt und wüsten Plätzen im Osten nicht selten oder zerstrent, im Westen sehr zerstreut, auf den Nordfriesischen Inseln nur auf Sylt: Keitum 1912!!.

Die erste Erwähnung findet $B$, arvensis durch Esmarch (Progr. Schlesw. I. 18. 71 [1789]). Hierher gehört B. commutatus Sickmann (Enumeratio 12 [1836]) nach Sonder.

\section{Formen.}

Neben der allgemein verbreiteten Rasse sind zwei fremde Rassen eingeschleppt selten beobachtet worden.

1. Größte Deckspelzen etwa $8 \mathrm{~mm}$ (oder etwas darüber) lang. Selten eingeschleppte Rassen .................. 2 - Größte Deckspelze nur $7 \mathrm{~mm}$ lang:

var. eu-arvensis A. 1. Gr. Syn. Mitt.-Europ. Fl. II. 1. 608 (1901). - Älrchen (1,2-)1,5-2 cm lang. — So fast ausschließlich bei uns. 
Dazu als Unterformen:

f. gracitis nov. $\mathrm{f}$. - Stengel niedrig, fein, mit einem bis drei Ährchen; Ährchen meistens dreiblütig. - Hamburg: Dampfmühle Wandsbek (Zimpel 1894!). Oldenburg: Neu-Teschendorf!! Kiel: Schulenhof (A. Christiansen!).

f. violaceus A. u. Gr. a. a. O. II. 1. 609 (1901). - Deckspelzen violett oder rotbräunlich überlaufen. - Hin und wieder beobachtete Farbenform.

2. Deckspelzen kahl:

var. Tyyatinus A. u. Gr. a. a. O. II. 1. 609 (1901), - Ährchen groß, bis $3 \mathrm{~cm}$ lang, mit fünf bis acht Blüten, an feinen, abstehenden oder zuletzt oft zurückgeschlagenen Ästen; Grannen oft auswärtsgebogen.

Bisher nur Kiel (A. Christiansen!).

Die Kieler Pflanze besitzt gestreckte Rispenäste, die größtenteils einährig sind und dadurch an var. fragilis erinnern. Übrigens zeigen herbstblühende Pflanzen ebenfalls öfter derart einährige (aber gestreckte) Äste (f. laxus A. u. Gr).

- Deckspelzen behaart:

var. velutims Duval-Jouve nach Hauskn. Bot. Ver. Thïr. N. F.

XIII. 54 (1899). — Rispenäste oberwärts und Hüllspelzen behaart.

Hamburg: bei der Dampfmühle Wandsbek (Zimpel $1894 !)$.

155. (sp). 66). Bromus racemosus L. Sinee plant. ed. 2. $11+(176 \div)$.

$\odot \odot$ und $\odot$. Stengel einzeln oder zu renigen, aufrecht oder aufsteigend, 0,2-0,8 m hoch, glatt, kahl oder unter den unteren Knoten zerstrent kurhaarig. Blattscheiden dicht kurzharig oder die wheren kahl. Blattfäche $2-5$ mm breit, am Rande rauh, heiderseits kurz helhart.

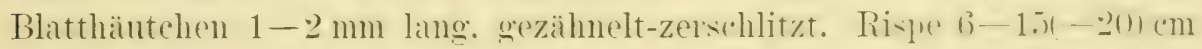
lang, nach dem Terblühen schmal, mit bis s cm langen. zuweilen etwas nickenden, meistens fast aufrechten. zwei his dreil grmulstundige Zweige

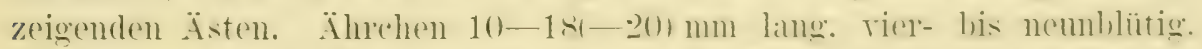
Hüllspelzen spitzlich, untere dreinervig. 5- 6 mm lang. where fünf- bis siebemervig. $7-8$ mm lang. Deckstelgen mit bogigem seitemrande. lis $7(-8,5) \mathrm{mm}$ lang, kahl oder fein rauh, mit bis $8 \mathrm{~mm}$ langer Granne. Antheren $2.5-3 \mathrm{~mm}$ lang. vielmals lïnger als breit. Blüte Mai lis . Tuli.

Auf Wiesen (oft angesäet), weniger oft auf Äckern (selbst unter

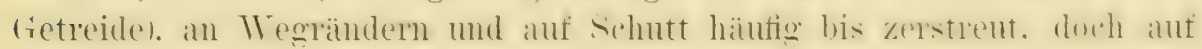


den Nordfriesischen Insehn nur anf Sylt (Schiötz): Timmum!! und Helgoland (Hallier).

Zuerst erwähnt für Holstein von Hornemann (Oec. Plantel. 3. Udg. 1. $123[1821])$.

\section{Formen.}

1. Unterste Rispenäste mit bis vier Ährchen und einem bis drei grundständigen Zweigen:

f. typicus nov. f. - So besonders auf fruchtbarem Boden, zerstreut. - Unterste Rispenäste mit einem bis zwei Ährchen und einem oder keinem grundständigen Zweige:

f. simplex Sonder Fl. Hamb. 68 (1851). - So an trockeneren Orten zerstreut. Eine Form mit dünnem, bis $0,4 \mathrm{~m}$ hohem Stengel und nur einem bis zwei Ährchen sammelte A. Christiansen im Ostenfelder Moor bei Rendsburg.

156. (.1). 67). Bromus hordeaceus L. spec. plant. ed. 1. 77 (1753).

$\odot$ und $\odot$. Stengel einzeln oder zu mehreren bis vielen, aufrecht oder anfsteigend, (3-120-6) (mo hoch, glatt, kahl oder (besonders an den Knot'n) behalart. Blattscheiden dicht weichharig, die oberen am Grunde zuweilen kahl. Blattfiache 2- 4 imm breit, an Rande raul, beiderseits dicht weichharrig. Blatthäutchen 1--2 mm lang, stumpt, gezähnelt. Rispe (2-)5-12 cm lang, dicht, zusammengezogen, ihre unteren Äste mit zwei bis vier Ährchen und $(0-) 1-4$ grundständigen Zweigen. Ährchen 8-20 mm lang, vier- bis zwölfblütig, spitzlich oder stumpf. Hüllspelzen spitzlich, untere (4-)5,5-7 mm, obere $(5-) 6-8 \mathrm{~mm}$ lang, drei- resp. siebennervig. Deckspelzen (4-j6,5-9 mm lang, siebennervig, dicht kurzhaarig, seltener kahl, mit bis $8 \mathrm{~mm}$ langer Granne. Vorspelze kiurzer als die Deckspelze. Antheren $1-1,5 \mathrm{~mm}$ lang, bis etwa doppelt so lang wie breit. Blüte Mai bis Juli, selten später.

Auf Wiesen und Triften, weniger oft auf Ïckem, vielfach an Wegrändern, auf Sandfeldern und auf Dünenboden, öfter auch auf Schutt; durch das Gebiet häufig, auch auf Fïhr, Amrum, Sylt, Röm, Nordstrand, Pellworm und Helgoland.

Weber erwähnt B. hordeaceus als B. mollis L. in Spec. plant. ed. 2 112 (1762) (Prim. Fl. Hols. 9 [1780]).

\section{Formen.}

Die Art zerfällt in zwei Rassen:

1. Stengel aufsteigend oder allseitig niederliegend, $3-15(-20) \mathrm{cm}$ lang, in der Regel zu vielen; Rispe kurz, dicht, ihre unteren Äste mit einem bis zwei Ährchen und $0-1(-2)$ grundständigen Zweigen, 
stets dicht, mit sich berïhrenden Ährchen; Ährehen $8-12(-15) \mathrm{mm}$ lang, stumpf, mit vier bis sechs Blïten; Deckspelzen bis $6,5 \mathrm{~mm}$ lang, meistens kahl; Antheren kaum $1 \mathrm{~mm}$ lang; katm länger als breit:

var. Thominii A. u. Gr. Wiss. Meeresunters. Nr. IV. 104 $(19)(1))$.

Auf Dïnen und Sandland, seltener auf Felsen, an der Ostsee verbreitet, an der Nordsee selten und bisher nur auf Helgoland (Hallier, Brody!).

Die Rasse wurde von Knuth (Allg. Bot. Zeit. IV. 110 [1898]) für Helgoland als $f$. Lloydiamus ohne Autorennamen aufgeführt, hat aber mit Serrafalcus Lloydianus Godr. u. Gren. nichts zu tun. Die früheren Floren unseres Gebiets nemnen sie als $f$. hordeaceus (nach Fries). Sie ist in typischer Ausbildung recht charakteristisch, doch sind die Merkmale nicht konstant. Kräftige Exemplare leiten in den Größenverbältnissen der einzehen T'eile in den 'Iypus über. Die Deckspelzen sind nicht immer kahl, sondern oft an Rande, nicht selten auch auf dem ganzen Rücken, behaart. Diesen Übergängen stehen manche Formen des Binnenlandes nahe, die sich von var. Thominii aber durch gestreckteren Stengel, weniger dichte und stärker verzweigte Rispe sowie öftere Spelzenbehaarung unterscheiden (so z. B. Sonder, Fl. Hamb. 68 [1851] und bei Hamburg auf Schutt [Jaap!]).

- Stengel aufrecht oder aufsteigend, (5-)20-60 $\mathrm{cm}$ hoch; Rispe meistens größer, lockerer, ihre unteren Äste mit bis vier Ährchen und bis vier grundständigen Zweigen; Ährchen (8-)15-20 mm lang, spitzlich, mit (vier bis) sechs bis zwölf Blüten; Deckspelzen bis $9 \mathrm{~mm}$ lang, mit längerer Gramme, behaart oder zuweilen kahl; Antheren etwa $1,5 \mathrm{~mm}$ lang, doppelt so lang wie breit:

var. vulgaris nov. var. - Typus der Art. Zerfällt in mehrere

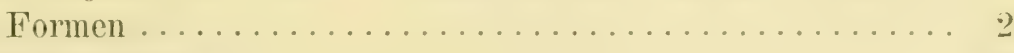

2. Deckspelzen behaart $\ldots \ldots \ldots \ldots \ldots \ldots \ldots \ldots \ldots \ldots \ldots \ldots$

- Deckspelzen kahl:

f. leptostachys Beck Fl. Nied.-Oesterr. I. 109 (1890). - So zerstreut, stellenweise nicht selten, auch auf Helgoland und Sylt!!.

Dazu als Unterform:

f. pseudoracemosus A. 11. Gr. Syn. Mitt.-Europ. Fl. II. 1. 616 (1901). -- Pflanze kräftig, bis $0,65 \mathrm{~m}$ hoch; Rispe reichährig, ihre unteren Äste mit vier grumdständigen Zweigen. - Hamburg: Hasselbrook (J. A. Schmidt 1869 !).

3. Hüllspelzen über 5 resp. $6 \mathrm{~mm} \mathrm{lang;} \mathrm{unterste} \mathrm{Deckspelze} 7-9 \mathrm{~mm}$ lang; Frucht kürzer als die Deckspelze ............. 4 
- Untere Hüllspelze etwa $4 \mathrm{~mm}$, obere etwa $5 \mathrm{~mm}$ lang; längste Deckspelzen etwa $5(-5,5) \mathrm{mm}$ lang, kürzer als die Frucht:

f. microstachyns Hackel in litt. (1913). - Ährchen 8-10 mm lang. - Kiel: Rotenhahn!, Viehburg!, Gaarden!, Heikendorf! (A. Christiansen als B. racemosus). - Eine auffällige, wohl weiter verbreitete Form, welche durch ihre Ährchen an $B$. brachystachys erinnert.

4. Rispe mit zahlreichen Ährchen

- Rispe mit wenigen (einem bis drei) Ährchen:

f. names A. u. Gr. a. a. O. II. 1.616 (1901). - So auf trockenem Boden nicht selten, oft in Menge; von Nolte schon 1825! auf Sylt gesammelt. Zuweilen mit kahlen Deckspelzen.

5. Rispe ziemlich locker, ihre mteren İte 2-8 cm lang, mehrährig:

f. typicus Beck a. a. O. I. 109 (1890). - Häufigste Form.

- Rispe sehr dicht, ihre unteren Äste nu 3-12 mm lang, oft (nicht immer) einährig:

f. confertus G. F. W. Meyer Chlor. Han. 614 (1836) (unter B. mollis). - So zerstreut, bei Hamburg schon 1870: Hammerbrook (J. A. Schmidt!).

\section{Mißbildungen.}

f. m. ramifloms nor. f. - Pflanze aus den Knoten mit rispentragenden Ästen. - Lübeck: Heidberg (Friedrich 1894!).

Eine auffällige Monstrosität, bei der einzelne Hüllspelzen tief gespalten sind, fand sich bei Kiel: Labö (A. Christiansen 1912!).

157. (sp. 68). Bromus commutatus Schrader Fl. Grem. I. 355 (1806). $\odot$ und $\odot$. Stengel einzeln oder zu mehreren. aufrecht, seltener aufsteigend, kahl oder an den Knoten behaart, $0, t-1 \mathrm{~m}$ hoch. Blattscheiden behaart, die oberen oft unterwärts kahl. Blattfäche $₹-5 \mathrm{~mm}$ breit, am Rande schwach rauh, beiderseits weich kurzhario. Blatthäutchen kurz. gezähnelt-zerschlitzt. Rispe $(5-112-20$ cm lang, sehr locker, mit bis $12 \mathrm{~cm}$ langen. bis sieben Ährchen tragenden, dünnen. nickenden bis überhängenden unteren Ästen mit bis fünf grundständigen Zweigen. Ährehen 15-20(-25) mm lang, 5-9(-13)blütig. Hüllspelzen spitz, untere 5-6, obere $7-8 \mathrm{~mm}$ lang. Deckispelzen $8-9 \mathrm{~mm}$ lang. rauh, die unterste mit etwa 5 mm langer, die übrigen mit bis 12 mm langer, gerader Gramne. Torspelze kürzer als die Dechispelze. Antheren bis $2 \mathrm{~mm} \mathrm{lang.} \mathrm{etwa}$ dreimal so lang wie breit. Blïte Juni bis September.

Auf Schuttland, weniger auf Wiesen, Äckern und an Wegrändern, sehr zelstrent und oft mbeständig. einheimisch nur aut Elbrorlandswiesen um Hamburg: 
La uenburg: zwischen Ammïhle und rilk (Laban 1895!). Hamburg: Elbrorland bei Warwisch (einheimisch)!!. Kuhwärder 1('. Timm 186s.

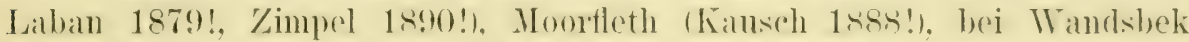
(.J. A. Schmidt 1867 !) ant dem Königsland und bed dor I)amptmiihle (Zimpel 1892!), Hammerbrook (Laban 1869! als B. patulus), Wilhelmsburg

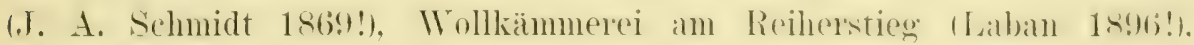
Georgswärder (Zimpel 1893!), Winterhude (J. A. Schmidt 1873!) mehrtach, Harvestehude (Laban 1879!), an Dielsteich in Altoma (Iaban

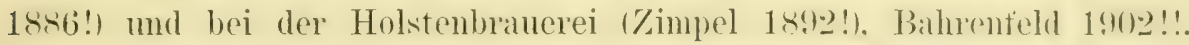

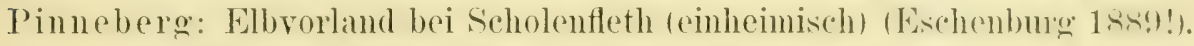
Sogeberg (Zimpel 1691!). Kicl: Hassee (Prahl!), beim Eirlerkrmg (Prahl 1886!), bei Brandsbek und Hot Krog (A. Christiansen!), Ellerbek ([oulsen!). Schleswig: zwischen Tiergarten und Chaussee (Himrichsen 185̆1!). Angeln: Ansacker (Hansen!). (quern (.Tensen 1870!). Helgoland (Gätle!).

Von einer Reihe genannter Standorte lagen keine Pflanzen vor: La unburg: Escheburg (Sonder, Fl. Hamb. 67 [1851]). Hamburg: Barmbeck und steilshop (Sickmamm, Enmmeratio 12 [1836]), Dammthor. Bergedorf und St. Pauli (Sonder a. a. O.), stadtgraben und St. Pauli (C. Timm Naturw. Ter. Hamb. N. F. IT. 80 [1880]), Uhlenhorst (C. T'imm a. a. 0.). Stormarn: Steinbek (Sonder a. a. 0.). Segeberg: Strenglin (Schmidt Naturw. Ter. schlesw.-Holst. III. 101 [1878]). Alsen: sonderburg (Schiötz) und Gumdestrup) (Petit Bot. Tidsskr. XII. 25 [1880]), Kjär (Petersen Progr. Sonderb. 45 [1891]). Hadersleben: Aaroe (Hansen nach Knuth Fl. v. Schlesw.-Holst. 781 [1887]).

Von Sonder gesammelt, liegt B. racemosus als B. commutatus im Kieler Herbar!. Was Hennings bei Kiel als $B$. commutatus sammelte, gehört zu $B$, racemosus!. Ein auf Alsen gesammeltes Exemplar rom Miangsee (Hb. Kopenhagen!) ist ebenfalls B. racemosics.

158. (adv. 77). Bromus japonicus Thunberg Fl. Tap. 52 t. 11 (1784).

๑. Stengel einzeln oder zu mehreren, meistens aufrecht, 0,2--0,6 m hoch, kahl oder an den Knoten behart. Blattscheiden behart oder die oberen kahl. Blattfläche 2-4 mm breit. kurzharig. Blatthäutchen 1-3 mm lang, zerschlitzt. Rispe glof, 10-15-20) cm liug. zuweilen liurzer, locker mit einseitig überhängenden Ästen: untere İste verlängert. mit zwei bis drei (bis vier) Ährchen und ein bis zwei (bis vier) grundständigen Isten. Ährehen 1.2-2-1-t) cm limg. sieben- bis zwölfblïtig. Hïllspelzen ungleich, untere 5-6. obere 7 mm lang. Deckspelzen lis. $9 \mathrm{~mm}$ lang. länglich-lanzettlich. wie die Hüllspelzen mit wenig deutlichen. nicht hervortretenden Nerven, mit an den unteren kurzer, $3-4 \mathrm{~mm}$ langer. gerader. an den oberen längerer. bis 12 mm langer. nach auben gekrimmer Gramme. zur Fruchtzeit nur am Grumde sich deckend. Fin- 
spelze kürzer als die Deckspelze. Antheren etwa $1,5 \mathrm{~mm}$ lang, etwa dreimal so lang wie breit. Blüte Juni und Juli, zuweilen später.

Auf Schutt selten.

Hamburg: bei Wandsbek auf dem Königsland (WV. Timm, Prahl 1891!: Dentsche Bot. (ies. X. 87 [1892]) und hei der Dimpfmühle (Zimpel 1893!, 1894!), Kuhwärder 1902!!, am Diebsteich in Altona (Dinklage, W. Hansen 1855!) Kiel: Veumühlen (Erichsen. Prahl 1887!), Labö (v. Fischer-Benzon, Prahl Krit. Fl. 261 [1890] als B. patulus M. u. K.). Tondern: Hoyer (Friderichsen. Hh. Kopenhagen!). - Andere Angaben (fuir Hamburg) beziehen sich auf $B$. squarrosus oder $B$. commutatus.

\section{Formen.}

Vom Typus weichen ab:

f. velutinus A. u. Gr. Syn. Mitt.-Europ. Fl. II. 1. 619 (1901). Deckspelzen dicht kurzhaarig. - Hamburg: Dampfmühle Wandsbek (Zimpel 1894!).

f. submonostachys nov. 犬. - Pflanze niedrig, zart, mit 1-2 Ährchen. - Mit voriger Form!.

159. radv. 78). Bromus squarrosus L. Spec. plant. ed. 1. 76 (1753). $\odot$ und ¿. Stengel zu mehreren, meistens aufrecht. $0.3-0,6 \mathrm{~m} \mathrm{hoch}$. kahl. Blattscheiden behaart oder die oberen kahl. Blattfläche $2--4 \mathrm{~mm}$ breit, zuweilen rom Rande schwach eingerollt. unterseits schwächer, oberseits stärker behaart. Blatthäutchen 1-2 mm lang, zerrissen. Rispe 16-110-20 cm lang. locker. mit bis $7 \mathrm{~cm}$ langen. $2(-3)$ Ährchen tragenden, mit bis vier grundständigen Zweigen rersehenen unteren Rispenästen; alle Äste überhängend oder abstehend. Ährchen $2-3(-4,5) \mathrm{cm}$ lang; mit $7-15(-19)$ Blüten. Hüllspelzen spitzlich. untere 5-6. obere 8-9 mm lang. Deckspelzen bis $10 \mathrm{~mm}$ lang. eiförmig-lanzettlich, wie die Hüllspelzen mit dentlich hervortretenden Nerren, mit an den unteren kurzer, :3-5 mm langer, gerader, an den oberen längerer, bis $11 \mathrm{~mm}$ langer, auswärts gebogener Gramne. Vorvpelze kürzer als die Deckspelze. Antheren $1 \mathrm{~mm}$ lang, doppelt so lang wie breit. Blüte Juni bis September.

Auf Schutt und an Wegen selten.

Hamburg: auf Kuhwärder am Köhlbrand (J. A. Schmidt 1868!, Zimpel 1887!), Kl. Grasbrook (Laban 1869!. J. Schmidt 1890!), Hammerbrook (Laban 1868 in $\mathrm{Hb}$. J. A. Schmidt!), bei Wandsbek auf dem Königsland (Prahl 1891!) und bei der Dampfmühle (.Jaly 1892!, seitdem fast jährlich) 1902!!, Sechiffbeker Mühle bei Horn (.T. A. Schmidt 1885!), Harrestehnde (Zimpel 1890!), Eppendorf (Laban 1889!), Tinterhuder Bruch (Zimpel 1890!), bei Altona am Diebsteich (C. Timm, Laban!) und 
bei der Holstenbranerei (Zimpel 18:41!). Langentelde (Labban 18!n)!.

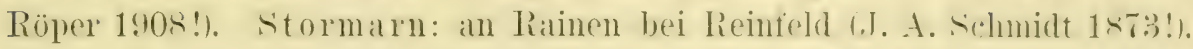

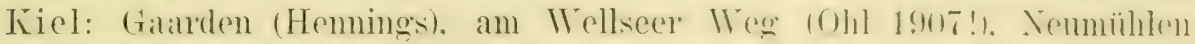
(A. Christiansen 1908!).

Zuerst erwåhnt von C. T'imm (Deutsche Bot. Ges. V. CIII [1887]).

Vom Typus weichen ab:

Formen.

f. pendulus nov. f. - Rispe bis 28 cm lang, locker, mit sehr verlängerten (bis $15 \mathrm{~cm}$ ) Ästen, welche an den ïberhängenden Enden der abstehenden Äste bis drei Ährchen tragen. — Kiel: auf Schntt bei Neumühlen (A. Christiansen 1908!).

f. villosus Koch Syn. Deutsch. u. Schweiz. Fl. ed. 1.821 (1837). Deckspelzen dicht weichharig: - Hamburg: bei Wandsbek auf dem Königsland (J. Schmidt 1892!) und bei der Dampfmühle (Jaap 1892!, Kausch 1900!, 1904!) 1902!!.

160. (atlv. 79). Bromus briziformis Fisch. 11. Mer. Ind. Hort. Petropol. III. $30(1836)$.

$\odot$. Stengel einzeln oder zu wenigen, $(0,1-) 0,2-0,6 \mathrm{~m}$ hoch, aufrecht orler seltener aufsteigend. glatt. Kahl odel an den Kinoten behart. Blattscheiden dicht kurzhaarig oder die oberen liahl. Blattfläthe 2- 8 mm lreit, beiderseits dicht behant, am Rande rauh. Blatthäutchen kurz. Ireit. gezähnelt. Rispe $4-15(-20) \mathrm{cm}$ lang. locker. mit rerlängerten. übellängenden Ästen. Ährchen $(1-12-2.5(-3.5)$ cm lang. mit $11-15(-21)$ Blüten. Hüllsvelzen stumpflich, untere 5-6. obere $7-8 \mathrm{~mm}$ laug. glatt. Deckspelzen bis 9 mm lang, tast eifürmig. gramenlos. Torspelze kiuzer als die Deckspelze.

Auf Schutt und Gartenland selten.

Ha mburg: Georgswärder (Zimpel 1894!). Dimnfmïhle Tiandsek A. Mohr 1904!). Winterhuder Bruch (.T. schmirlt 1890!: Desutsoh. Bot. (tes. IX (126) [1891]), Booth Garten in Flottbek (C. Timm. IT. Hansen 1886!) (Laban Fl. v. Hamb. 4. Antl. 198 [1887] als L. lingsuintes Lamk.). Steilshop $1912 ! !$.

Das Gras ist ferner ron Eppendorf, Isestraße, genamut worden (Ver. Naturw. Unterh. VII. 97 [1890]), doch fehlen Belege. Ohl neunt es (Pthanz. Umg. Kiels 22 [1889]) als rerwildert auf dem neuen Elmschenhagener Kirchhof.

Formen.

Vom Typus weicht geringfïgig ab:

$f$. monostachys nov. f. - Stengel fast haarfein, mit eimem $1-1, \tilde{c} \mathrm{~cm}$ langen Ihrchen. - H a m bu $\mathrm{r}^{*}$ : Steilshop!!. 
161. adr. 80). Bromus scoparius L. Amren. acad. IT. 266 11759\%.

¿ und -.. Stengel zu mehreren bis rielen. autrecht oder aufsteigend, (1.1-0.3 m hoch. kahl. Blattscheiden dicht bis zerstrent abstehend behart. Blattfiache 2-3 mm breit. unterseits fast kahl, oberseits kurz behant. gegenden firund und am Rande länger gewimpert. Blatthäutchen kurz. oft fast fehlend. Risue 3-5 cm lang. dicht. mit zusammengezogenen Ästen: Äste mit einem bis zwei Ïhrchen. $1-6 \mathrm{~mm}$ lang. B̈hrehen 8 bis $13 \mathrm{~mm}$ lang. fünt- lis acht- (bis zehn-1hlütig. Hüllspelzen spitz. untere 5-6. obere 6-7 mm lang. kahl oder behaart. Deckspelzen bis $7,5 \mathrm{~mm}$ lang. kahl oder behaart. tief zweizähnig. deutlich hautrandig. mit $3-8 \mathrm{~mm}$ langer Grame. Torspelze kürzer als die Deckslelze. Blüte Mai bis .Juli.

Auf Schutt bei Hamburg selten.

Hamburg: bei der Wollkämmerei am Reiherstieg IZimpel 1894!. .T. Schmidt 18.96!: Deutsche Bot. M[nnatsichr. XVI. 115 [1808] mit Autorangahe ..s.": wohl Drucktehlerı. Dampinühle Wandsbek (.J. Schmidt 1894!). am Diebsteich in Altma 1C. Timm. Laban 1sxib!: Prahl hirit. FI. 261 [1890] als B. confertus M. B.).

Vom Typus weicht ab:

\section{Formen.}

f. glabratus nov. f. - Hüll- und Deckspelzen kahl. - Hamburg: Dampfimühle Wandsbek (J. Schmidt!).

162. (adv. 81). Bromus macrostachys Desfont. Fl. Atlant. I. 96 (1798). ¿. Stengel zu mehreren. autsteigend. $0.2-0.5 \mathrm{~m}$ hoch. glatt, kahl. Blattscheiden dicht weichharig oder die oberen oft kahl. Blattfläche $2-3(-4) \mathrm{mm}$ breit. am Rande rauh. beiderseits zerstreut kurzharig. Blatthäutchen his $1 \mathrm{~mm}$ lang. gestutzt. Rispe dicht. 3-81-151 cm lang. mit kurzen. bis $5 \mathrm{~mm}$ langen. einährigen. starren Ästen. Ährchen 2-3 cm lang; mit 8-15 Blüten. Hüllspelzen dicht behaart, untere $6-7$, obere $8-9 \mathrm{~mm}$ lang. Deckspelzen bis $12 \mathrm{~mm}$ lang. undentlich nerrig. unterwärts kahl, mach oben dicht behaart. tief zweizähnig. aus dem Einschnitt tim oberen Drittelı mit 5-15 mm langer. stark auswärts gebogener. nach oben gekrümmter Gramne. Torspelze kürzer als die Deckspelze. Blüte.Juni bis August.

Auf Schutt selten.

Hamlurg: bei der Wollkämmerei am Reiherstieg IC. Kansch 1896!: Deutsche Bot. Monatsischr. XVI. 115 [18!s] mit Autorbezeichmung ..Par-

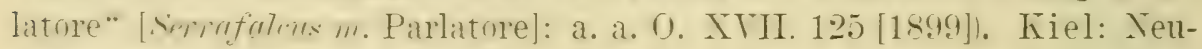
mühlen (A. Christiansen 1909!).

Die Pflanzen mehrerer Hamburger Herbarien, als B. macrostachys bezeichnet, sind B. squarosus!, ron ersterer Art sofort zu unterscheiden durch den deutlichen Deckspelzenwinkel. Die Seitenränder der Deckspelzen rerlaufen bei B. macrostachys bogig. Zu B. squarosus gehört auch eine als $B . m$. bezeichnete Pflanze rom Kil. Grasbrook! (Laban nach Ver. Naturw. Unterh. Hamb. VII. 66 [1894]). 
163. (atdv. 82). Bromus uniolioides Humb. u. Kunth Nov. (ien. I. 151 (1815).

$\odot \odot(0 b$ bei uns?). Stengel zu mehreren, aufrecht oder aufsteigend, 0,3-0,8 m hoch, glatt. Blattscheiden glatt, kahl oder die miteren oder alle weichharrig. Blattfäche $2-7 \mathrm{~mm}$ breit. beiderseits (oft stark) rauh. fein gewimpert. Blatthäutchen 2-3 mm lang, stumpt. beiderseits oft behaart. Rispe 8-20 cm lang. mit anfrecht zusanmmengerenem order aufrecht abstehenden, bis $7 \mathrm{~cm}$ langen, mehrährigen $\ddot{A s t e n ~ m i t ~ b i s ~ v i e n ~}$ grundständigen Zweigen. Ährehen seitlich zusanmengerlürekt. zweischneidig, (1,5-)2-3.7 cm lang, fünf- bis nemblïtig. Hüllspelzen spit\%, am Kiele rauh, untere 9-10, obere $11-13 \mathrm{~mm}$ lang. Deckspelzen spitz, grannenlos oder selten bis $3 \mathrm{~mm}$ lang begrannt, bis $16 \mathrm{~mm}$ lang. oberwärts an den Nerven rauh. Vorspelze kaum zwei Drittel so lang wie die Deckspelze. Blüte Juni bis Oktober.

Auf Schutt selten.

Hamburg: Wollkïmmerei am Reiherstieg (Zimpel 1893!. spüter mehrfach!)!!, Georgswärder (Zimpel 1893!), Kl. Grasbrook (Laban 1894!), Hammerbrook (J. Schmidt, Deutsch. Bot. (Ges. VII. (94). [1889]). Dampfmühle Wandsbek (Zimpel 1894!)!!, Winterhuder Bruch (J. Schmidt 1889!). Steilshop 1912!!, Fuhlsbüttel (.T. Schmidt 1912)!!, Eppendorf (.Taap 1889!!). am Diebsteich in Altona (.T. Schmidt 1889!), bei der Holstenbranerei (Zimpel 1891!), Bahrenfeld 1900, 1904!!, Gr. Flottbek (Zimpel 1895!). Kiel (A. Christiansen 1906!).

\section{Formen.}

Vom Typus weichen ab:

f. abtrevicutus Hauskn. Deutsche Bot. Monatsschr. XVI. 115 (1898). - Rispenäste verkürzt, daher die Rispe schmal, zusammengezogen. - H a mburg: Wollkämmerei am Reiherstieg (Zimpel $1894-96 !)$

f. aristatus nov. f. - Deckspelze mit 2-3 mm langer Granne. H a mburg: Georgswärder (Zimpel 1894!).

\section{Gattung.}

\section{Brachypodium.}

Pal. Beaur. Agrost. 100 (1812).

1. Grame wenigstens der oberen Deckspelzen länger als ihre Spelzen.

- Alle Grannen kürzer als ihre Declispelze ................ pinnatum.

2. Pflanze 2, mit kriechender Grundachse; Ähre vielälırig .......B. silvaticum.

- Pflanze $\odot$, mehrstengelig; Ähre mit einem bis drei Ährchen... B. distachyon. 
164. (.1) 1)<1. Brachypodium pinnatum Pal. Beaur. Agrest. 155 (1812). 7. Crmularhe kierehend. Stengel aufrecht oder iofter aufsteigend, 0.4-1,2 m hoch, glatt oder schwach rückwärts rauh, an und oft auch muter den knoten (besonders Ifen muterens dicht zottig behart. Blattscheiden glatt oder schwach rauh, untere dicht behart, obere kahl. Blattfärohe 3-8 mm breit. muterseits und am liande behart, am Rande und oberseits ranh. Blatthäutchen etwa $1 \mathrm{~mm}$ langr. breit. zerschlitzt.

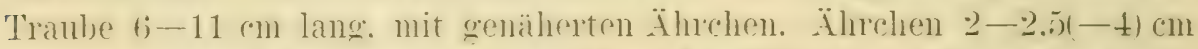

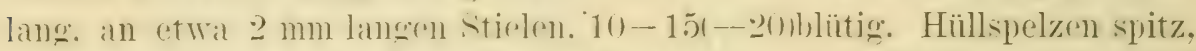

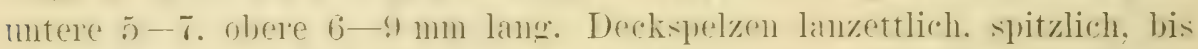

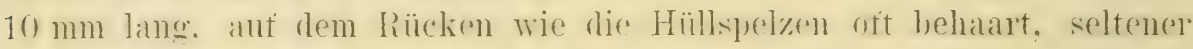
kahl, mit bis $5 \mathrm{~mm}$ langer Granne. Antheren rötlich.

In Wäldern und Gebïschen selten.

Lauenburg: bei Lauenburg (Nolte, Hansen 1847!) im Fürsten-

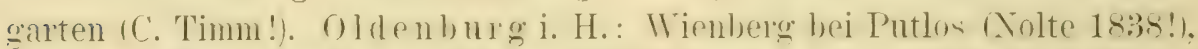

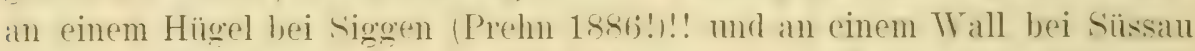
(Prehn). Hamburg: bei der Wullkämmerei am Reiherstiers (Zimpel!). Plön: auf einer Wiese am See nach Ascheberg hin (Schultz 1894!. Hb. Prahl). Kiel: an den schiobsanden bei Kielerhof (Prahl 1887!), am Kanal bei Knoop (A. Christiansen 1907!).

Bereits Weber nennt Bromus pinnatus L. für Holstein, doch ist seine Pflanze nicht B. pinnatum, sondern Bromus pinnatus $\beta$. L. (ebenso bei Deder Fl. Danica t. 164 [1764]), und damit gleich Brachypodium silvaticum; bei Weber führt diese Art die Bezeichnung Triticum bromoides (Prim. Fl. Hols. 11 [1780]). Das Indigenat der Pflanze in unserm Gebiet ist zweifelhaft, am sichersten für die Oldenburger Fundorte, unsicher für Lauenbur@. Bei Hamburg, Plön und Kiel ist Verschleppung sicher.

Eine grobe Reihe von Notizen ist unrichtig resp. unsicher. Wenn Knuth (Fl. v. Schlesw.-Holst. 779 [1887]) Neustadt und Apemrade nennt, so ist der Grund Verwechslung von B. pinnatum mit B. silvaticum (Nolte!, Bargum!). Dieselbe Verwechslung dürfte für den Fundort Burg a. F. vorliegen; Belege fehlen. Vom hohen Elbufer (Sickmann

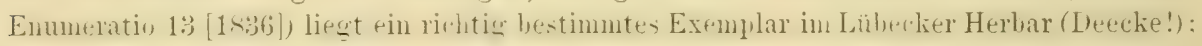
Sonder und andere Floristen beobachteten indes $B$. pinnatum weder hier noch sonst bei Hamburg, trotz Hübeners Bemerkung, dab es zerstreut und nicht selten auftrete; ein Exemplar von Braunwald, gefunden bei "Hamburg", ist B. silvaticum!. Auch auf Alsen wächst nur B. silvaticum. Um diese Spezies handelt es sich auch bei Klatt (Fl. v. Lauenb. 160 [1865]) „am Juliusburger Steig".

Formen.

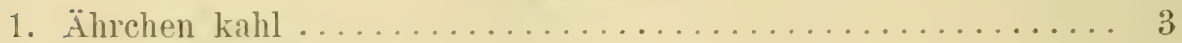

- Ährchen behaart:

f. vulgare Koch Syn. Deutsch. u. Schweiz. Fl. ed. 1. 818 (1837). - So an allen Orten. - Ährchen derselben Tranbenseite einander nur mit den Grannen erreichend oder wenig übereinandergreifend. 
Dazil als Unterform:

f. Totiaceum A. u. Gr. Syn. Mitt.-Europ. F1. II. 1. 633 (1901). - Ährchen derselben Traubenseite halb oder mehr übereinandergreifend. - O l d e n b u r g: Siggen!!. - Mehrfach in Übergängen.

2. Deckspelzen mit scharf abgesetzter, kurzer Granne:

f. gracile Pospichal Fl. Oesterr. Küstenl. I. 137 (1898). Ähchen rundlich. - Selten und mu verschleppt. Kiel: Knoop (A. Christiansen!).

- Deckspelzen fast gleichmäßig in die Granne verschmälert; Granne kurz, zuweilen fast fehlend, derb:

f. rupestre Rchb. Jcon. I. 6. fig. 1376 (1834). - Ährchen etwas zusammengedrückt. - Wie vorige Form nur verschleppt. Hamburg: Wollkämmerei Reiherstieg' (Zimpel!: dazn Triticum panormitanum in Deutsche Bot. Monatssche. XVI. 115 [1898]).

165. (sp). 70). Brachypodium silvaticum Roem. u. Shehult. Sist.II. $7+1 \mid 1 \times 17$.

4. Grundachse kurz kriechend. Stengel meistens anfrecht, 0, boch. glatt, unterwärts dicht, oberwärts zerstreut behart, unter der Rispe kahl, zuweilen fast ganz kahl. Blattscheiden mehr oder weniger raulıharrig. Blattfläche 3-10 mm breit, beiderseits dbesonders oberseits ranil. beiderseits an den Nerven rauhhaarig. Blatthäutchen 2-4 mm lang; stmmpf, gezähnelt. Traube bis ïber 20 cm lang. oft etwats überhängent. ت̈hrehen 2-3(-4) cm lang, 8-13(-15)blitig. Hüllspelzen spitz. behant oder kahl, untere $8-10$, obere $12-14 \mathrm{~mm}$ lang. Deckspelzen bis $12 \mathrm{~mm}$ lang, lanzettlich, behaart, seltener kahl, mit 1-2 cm langer Gramne. Antheren gelblich.

In Wäldern, Gebüschen und an Abhängen im Osten nicht selten, im Westen nur bei Hadersleben: Toftlund (Prahl), Gramm (Prahl)!!.

Zu dieser Art gehören Triticum bromoides Weber Prim. Fl. Hols. 11 (1780), Bromms pimutus Oeder Flora Danica t. $16+11764 t$ und Festum gracilis Hornemann Oec. Plantel. 3. Udg. 1. 120 (1821).

1. Ährchen behaart:

\section{Formen.}

f. dumosum Beck Fl. Nied.-Österr. I. 110 (1890), - Häufigste Form. Dazu als Unterform:

f. majus Lange Haandb. Dansk. Fl. 4. Udg. 50 (1886). Ährchen 3-4 cm lang, reichblütig. - So zerstreut auf fruchtbarem Boden, auch bei Gramm (Vilandt nach Lange). 
- Ährchen kahl odler die Dechspelzen nur oberwïrts kurz gewimpert: f. typicum Beck a. a. O. I. 110 (1890). -- Zerstreut.

\section{Mißbildungen.}

f. m. ramosum nov. f. - Einer oder mehrere der untersten Traubenaste rerlängert und am Grunde rerzeigt, mit zwei bis drei Ahrchen. L a uenburg: Bäk bei Ratzolurg (Zimpel 1900!). Ha mburg: Nienstedten!!. Kiel: Hochschar!. Eckernförde: Borghorst! und Wohlstorf! (A. Christiansen).

f. m. Trarteretum nor. 1. - Cnterstes Ährchen mit bis 9 cm langem Tragblatt. - Eckernförde: Borghorst und Wohlstorf (A. Christiansen!).

166. (adr. .3). Brachypodium distachyon Rioem. 11. Schult. Syst. II. 741(1817).

$\odot$. Stengel zu mehreren, am Grunde oft verzweigt, aufrecht oder aufsteigend. "1.1-0.3 $\mathrm{m}$ hoch, an den Funsten oft behart. Blattscheiden kahl, glatt. Blattfläche $1-2(-3)$ mm beit, oberseits stärker, moterseits schwächer rathharig. Blatthäntchen etwa $1 \mathrm{~mm}$ lang. stumpf. Tranbe meistens ein- bis dreiährig. Ährohen etwa $2(-3)$ cm lang, fast ungestielt, 6-15̄blütig. Hüllspelzen schmal, spitz, untere 5-6, obere $6-7 \mathrm{~mm}$ lang. Dechspelzen 7 - ! mm lang, kahl, sehr undeutlich genert, mit 7-12(-15) mm langer Granne. Blüte Juni bis September.

Auf Schutt selten.

H a ml,urg: Wollkämmerei am Reiherstieg (Zimpel 1895!, 1896!), Jampfniüle Wandibek (.J. Schmirlt Deutsch. Bot. Mronatsschr. XIT. 53 [1896]). Kiel: Neumühlen (A. Christiansen 1908!).

Tom Typus weicht ab:

\section{Formen.}

f. monostachyum Gussone Fl. Sic. Syn. I. 72 (1827). - Stengel nur 5-12 cm hoch, einährig; Ährchen drei- bis achtblütig. So am ersten Standorte (Zimpel 1895!).

\section{Gattung.}

\section{Triticum.}

L. Gen. plant. ed. 5. 37 (1754).

1. Hüllspelzen mit einem deutlichen Nerven (daneben zuweilen mit einigen un-

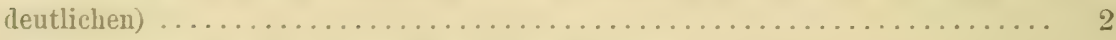

- Hüllspelzen mit drei oder mehr deutlichen Nerren ................ 6

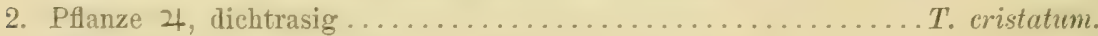

- Pflanze $\odot$, ein- oder melırstengelig $\ldots \ldots \ldots \ldots \ldots \ldots \ldots \ldots \ldots \ldots \ldots \ldots \ldots$ 
3. Rand der Hüllspelzen hart, knorpelig verdickt................. 4

- Rand der Hüllspelzen nicht verdickt..................... 5

4. Hüllspelzen kahl, von halber Ährchenlänge............T. mostratum.

- Hüllspelzen behaart, etwa von Ährchenlänge .............. orientale.

5. Hüllspelzen mit zwei Kielen........................ villosum.

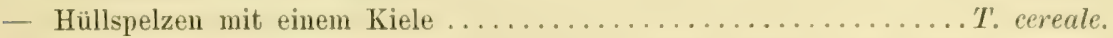

6. Pflanze 4; Deckspelze am Grunde mit einem etwa $1 \mathrm{~mm}$ breiten, harten

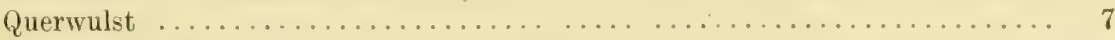

- Pflanze $\odot$ oller $\odot$; Deckspelze ohne Wulst .................. 9

7. Grundachse kriechend; Granne, wenn vorhanden, kürzer als ihre Deckspelze... 8

- Grundachse rasig; Grame zart, so lang wie die Spelze oder länger...T. caninum.

8. Ährenachse zäh; Deckspelzen spitz oder begrannt ............. repens.

- Ährenachse sehr brüchig, Deckspelzen stumpf ............. Tunceum.

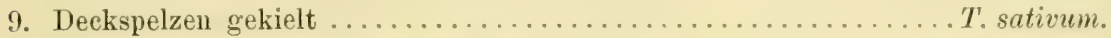

- Deckspelzen ungekielt, am Rücken abgerundet .................. 10

10. Hüllspelzen mit einer bis vier Grannen ..................... 11

- Hüllspelzen unbegrannt ......................... speltoides.

11. Hüllspelzen mit einer Grame .................. cylindricum.

- Hüllspelzen mit zwei bis vier Grannen ... . . . . . . . . . . . . . . . . 12

12. Hüllspelzengranmen der oberen Ährchen etwa $2 \mathrm{~cm} \operatorname{lang} . \ldots \ldots \ldots$. ovatum.

- Hüllspelzengrannen der oberen Ährchen bis $8 \mathrm{~cm}$ lang......... T. triunciale.

167. (sp. 71). Triticum caninum L. Spec. plant. ed. 1. 86 (1753).

4. Grundachse dichtrasig. Stengel zu mehreren, aufrecht oder aufsteigend, 0,6-1,2(-1,8) m hoch, glatt, kahl oder an den Kunoten rauh. Blattscheiden in der Regel kahl, glatt, selten behart oder schwach rauh. Blattfäche $4-8(-10) \mathrm{mm}$ breit, am Rande deutlich rauh, oberseits kurzhaarig. Blatthäutchen bis $1 \mathrm{~mm}$ lang, stunıf. Ähre $(4-) 10-15(-20) \mathrm{cm}$ lang, mit dem oberen Teile des Stengels oft ïberhängend. Ährchen (8-)10-18(-20) mm lang, zwei- bis sieben- (bis nenn-)blütig. Hüllspelzen spitz, wenig ungleich, untere dreinervig, $9 \mathrm{~mm}$ lang, obere fünfuervig, $10 \mathrm{~mm}$ lang, beide rauh. Deckspelzen bis $10 \mathrm{~mm}$ lang, schwach fünfnervig, unterwärts glatt, glänzend, oberwärts rauh, mit 10-18 mm linger Granne. Blüte Juni und Juli, zuweilen bis September.

In schattigen Laubwäldern; im Ostseegebiet zerstrent, südwärts selten bis Hamburg, im Testen nur bei Itzehoe und im nordwestlichen Schleswig; bei Hamburg ganz vereinzelt auf Schutt.

Zuerst erwähnt von Weber (Prim. Fl. Hols. 11 [1780]) als Elymus canimes.

Lauenburg: an Schallsee bei Hakendort 1912!!. im sachsenwalde (Nolte 1824!) bei der Aumühle (J. A. Schmidt 1864!) sowie zwischen Kupfermïhle und stangenteich 1904!! und im (iehege Witzhaver Berg 1912!!. Hamburg: Schuttplatz bei Bahrenfeld 1900!!. Stormarn: Ahrensburg (Sonder). Siegeberg: Strenglin und Pronstorf (.T. Schmidt), Gründe bei Goldenbek 1.J. Schmidt 1892!). Oldenburg: Fellenhusen und 
Dillme (Kansch». Koselau (Prehnl. Farve (.J. Schmidt 1892!) 1901!!. Lütjenlurg: Kletkamp und Blekendorf (Prehn. Hb. Borchmann!). Kiel: Bordesholm mach Hemnings). Yeumühlen (Heming:). Knoop (Nolte 1829!). (irttonf (Engelken). Eckernförde (Prahl): Behrenbronk (A. Christiansen 1!12!). Schleswig: im Tiergarten (Thm!. Hansen 1832!). Angeln: I.angralligan (Hansen 18158!). Westerholz und Cnewattholz 1911!!. Flens-

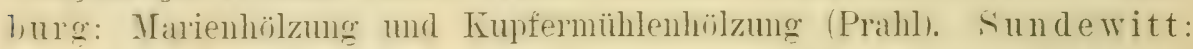
Broacker 1A. Christiansen 19og!!. Satrupholz (H. Petersen. Alsen: Fühnmallatf (Petitı. A le nla de: im .Jelm (Prahl)!! und Süderheissel!!,

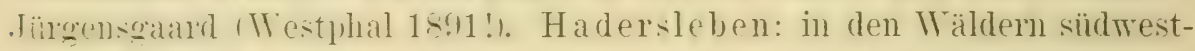

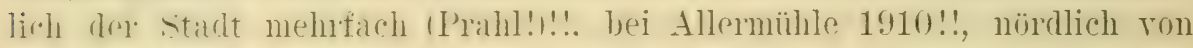

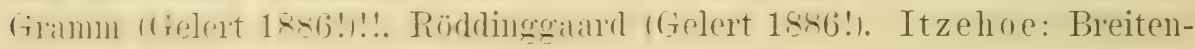
burg (Nolte 1822!).

Hornemann erwälnt T. caninum von Ratzeburg (Oec. Plantel. 3. Udg. 2. 126 [1837]); das Gras wurde hier bisher nur auferhalb des Gebiets gefunden: zwischen Römnitz und Kalkhütte (C. Schmidt) $1912 ! !$

Yößler nennt T. caninum ron Hamburg (Handb. Gewächsk. 1533 [1815]), Hübener (Fl. r. Hamb. 501 [1817]) von Pinneberg und Wohldorf, Laban (Fl. v. Hamb. 4. Aufl. 189 [1887]) ron Reinbek, Bergedorf und Eppendorf. Alle Angaben sind unsicher oder unrichtig, wie auch die ron A. Junge (Ver. Naturw. Unterh. Hamb. VIII. 99 [1890]) ron Winterhude und Altona auf Schutt; Belege ron ersterem Orte, 1870 gesammelt, gehören zu Brachypodium silvaticum!. Bei Lauenburg hat Claudius ein Gras gesammelt, das er selbst als fragliches T. canimum erwähnt; Klatt führt es (Fl. r. Lauenb. 161 [1860̃]) als diese Art auf. Für Lübeck liegt eine Angabe ron Häcker nach Brehmer vor; das Belegexemplar ist T. repens! (rgl. Friedrich Fl. т. Lüb. 44 [1895]). Unsicher sind weiter in

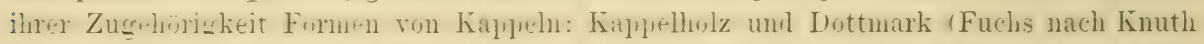
Fl. v. Schlesw.-Holst. 786 [1887]), ron Sonderburg (Wüstnei nach Knuth a. a. 0.), sowie ron Hemstedt (riünwald) und Heide (Sierchs und Hemnings) (beile nach Knuth a. a. 0.).

Formen.

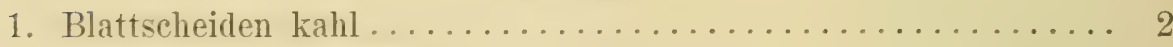

- Blattscheiden behaart.

f. Alexuosum Harz Bot. Centralbl. XLT. 10כ̃ (1891). - Zerstreut. Lauenburg: Hakendorf!!. Hamburg: Bahrenfeld!!. Segeberg: Goldenbek (J. Schmidt!). Kiel: Knoop! und Eckernförde: Behrenbrook! (A. Christiansen). Schleswig (L. Hansen 1832!, Herb. Schlesw.-Holst.-Lauenb. Fl. 211 [1835] z. T.). Angeln: Langballigan (Hansen!). Hadersleben: Pamhoeler Wald (A. Christiansen!). Zuweilen sind nur die Knoten behaart, so z. B. bei Westerholz!!.

2. Pflanze kräftig; Ährchen vier- bis sechsblütig.

f. typrimem A. u. Gr. Syrn. Mitt.-Europ). Fl. II. 1. 642 (1901). - Verbreitetste Form. 
Gewöhnlich gehören zu dieser Form als Abarten:

f. glaucum Hackel in Celak. Prodr. Fl. Böhm. 728 (1881).

- Ahre aufrecht; Pflanze grangrü. - An somnigen Orten; im Gebiet nicht festgestellt. - Dic Kombination dieser Abart mit $f$. flexuosm ist $f$. glaucescens Lange: Rördinggaard (Gelert!).

f. dasyrrhachis Celak. Böhm. Ges. Wiss. 621 [1887]. Ärchenachse an der dem Ährehen abgewandten, gewölbten Seite dicht kurzharig. - Eckernförde: Bor'óhorst und Behrenbrook(A.Christiansen!). Ha d e rs le ben : Allermühle!!. - Übergänge: Bahrenfeld und Granm!!. f. variegatus nov. f. - Hüll- und Deckspelzen riolett überlanfen. - Ha dersleben: Allermïhle!!. - Die gleiche Form in Ostpreußen: Ragnit: Wischwill!!

- Pflanze zierlich; Ähre kurz; Ährchen (zwei- bis) dreiblütig:

f. subtriflorm Parl. Fl. Ital. I. 495 (1848). - La uen burg: Hakendorf!!. Oldenbulg: Farve!!. Kiel: Knoop! und Eckernförde: Borghorst! (A. Christiansen). Angeln: Westerholz!!. Ha ders le ben: Fredstedt (A. Christiansen!). Gramm (Gelert!). - Anscheinend ist f. macitius Lange nicht wesentlich rerschieden.

Triticum panormitanum Bertol. Fl. Ital. IV. 780 (1839) ist entgegen einer Veröffentlichung für Hamburg (Deutsch. Bot. Monatsschr. XVI. 115 [1898]) in unserer Adventivflora nicht beobachtet worden. Die betreffenden Pflanzen gehören zu Brachyporlium pinnatum var. mpestre.

168. (sp). 72). Triticum repens L. spec. Plant. e(l. 1. s(; (175:3) subsu. eu-repens A. u. Gr. Syn. Mitt.-Europ. Fl. II. 1. 645 (1901).

4. Grundachse kriechend, mit oft langen Ausläufern. Stengel $10,2-10,5-1.2(-2)$ m hoch, glatt, kahl, aufrecht oder aufsteigend. Blattscheiden glatt, kahl oder die muteren behatrt. Blattfläche(2-14-9(--20)mm breit, beiderseits oder nur oberseits von Höckern und (weniger oft) ant den Verven einreihig stehenden Härchen lanh, am Rande stark ramh. Blatthäutchen kurz, oft fast fehlend. Ihre $6-15(-30) \mathrm{cm}$ lang: aufiecht. Alnchen 0.9-2.2 cm lang, drei- bis zehnblïtig. Hüllspelzen gramenartig spitz ausgezogen mit $1-3 \mathrm{~mm}$ langer Spitze, untere $8-10(-15)$, obere 9-11(-16) mm lang. fünf-bis siebemervig. Deckipelzen bis 13 mm lang. undentlich nervig; kahl, stachelspitzig oder mit bis 10 mm langer tirame. seltener stumpflich. Blüte Juni bis August, einzeln später.

Auf Äckern und Feldern. in Mäldern mo Giebüschen. auf sandland und Dünenboden gemein bis häufig; auch auf den Nordseeinseln.

Erwähnt von Weber (Prim. Fl. Hols. 11 [1780]). 


\section{Formen.}

1. Pflanze hell- bis dunkelgrïn, unbereift............... 2

- Pflanze graugrün, mehr oder weniger bläulich bereift........ 6

2. Alle Blattscheiden kahl ...................... 3

- Untere (oder alle) Blattscheiden behaart:

var. caesium Bolle Bot. Ver. Brandenb. II. 57 (1860). - Pflanze 0,4-1 m hoch; Stengel aufrecht oder am Grunde knickig niederliegend; Blattfläche $(3-) 5-9 \mathrm{~mm}$ breit, ziemlich schlaff, stark rauh; Ähre etwa 8-10 cm lang, selten länger; Ährchen 10-15 mm lang; Deckspelze mit (1-) $5-10 \mathrm{~mm}$ langer Granne.

So z. B. Lauenburg: Dermin!!. Hamburg: Winterhude (Jaap 1894!), Kuhwärder (Hansen 1891!), Eppendorf (Pieper!), Bergedorf (J. A. Schmidt 1864!), Hamm (J. A. Schmidt!). Pinneberg: Blankenese (Prahl!), Mühlenberg!!. St ormarn: Wandsbek (Zimpel!), Ahrensburg!!. Lübeck mehrfach (Friedrich usw.!): Wesloe (Prahl!). Segeberg!!: Högersdorf (J. Schmidt!). Plön: Timmdorf und Gremsmühlen!!. Kiel: Westensee! (A. Christiansen). Husum: Ahrenviöl (A. Christiansen!). Eiderstedt: Süderhöft (F. v. Müller). Angeln: Bokholmwik (Hansen). Hadersleben: Viktoriabad. (Prahl). (An diesen beiden Orten f. viride Marsson Fl. Neuvorp. Rügen 599 [1869]). Sylt: Westerland!!

3. Stengel $0,5-1(-1.5) \mathrm{m}$ hoch, aufrecht, ziemlich düm; Blattfläche $4-8(-10) \mathrm{mm}$ breit; Ähre (5-) $8-12 \mathrm{~cm}$ lang; Ährchen mit stumpfen oder spitzen bis lang begrannten Spelzen..........

- Stengel 1,2-2 m hoch, aufrecht, derb; Blattfläche 1-1,5(-2) cm breit; Ähre 15-20(-30) cm lang; Ährchen sehr zahlreich, bis $22 \mathrm{~mm}$ lang, mit stumpfen oder kurz stachelspitzigen Deckspelzen: var. majus Döll Rhein. Fl. 69 (1843). - So in feuchten Gebüschen und an Ufern besonders im östlichen Gebiet nicht selten, sonst zerstreut, auf den Nordseeinseln nur auf Helg ol and: Kirchhof!!.

4. Ährenachse kahl

-- Ährenachse behaart:

f. pubescens Döll Fl. v. Baden 129 (1857) erw. - Keine einheitliche Form, sondern mit verschiedenen Abarten kombiniert. Hierher Hansen Herb. Schlesw.-Holst.-Lamenb. Fl. 213 z. T.

Lauenburg: Techin (bei $f$. arvense)!!. Hamburg: am Stadtgraben vor dem Dammtor (Sonder; Döll a. a. 0.), 
Hammerbrook (bei f. arvense) (Jaap 1894!), Allermöhe (bei $f$. aristatum)!!. Pinneberg: Stellingen (bei $f$. arvense und f. Leersianum)!!. S egeberg (bei f. caesium)!!: Högersdorf (bei $f$. Vaillantianum)!!. Lü be ck: Schwartau (Friedrich!), Schlutup (bei $f$. aristatum)!!. Neustadt (J. Lange!) (bei f. majus). Plön: 'Timmdorf und Gremsmühlen (bei $f$. caesinm)!:. Oldenburg: Neukirchen (bei f. vulgare)!!, Heiligenhafen mehrfach!!, Großenbrode (bei f. majus)!!. Fehmarn: Schlagsdorf!! Kiel (bei $f$. caesium) (A. Christiansen!). Eckernförde (J. A. Schmidt!) (bei f. Vaillantianum). A ngeln: Beverö (bei f. maritimum) (Hansen!), Husby (bei $f$. Leersianum) (Hansen!), Holnis und Langballigholz (bei $f$. glaucum)!!, am Drei (bei $f$. collinum)!!, Glücksburg (bei $f$. muticum)!!, Kielseng (bei $f$. subulatum) (Gelert 1895!). Sundewitt: Stranderott (bei $f$. muticum, $f$. aristatum und $f$. glaucum)!!; Süderhaff (bei $f$. muticum)!!. Apenrade: Aubek!!. Hadersleben: Viktoriabad (bei f. cclesium) (Prahl!), Aarösund (Prahl), Gravenshoved (bei f. majus)!!, Wonsbek (Friderichsen), Föhr: Wyk (bei Ubergang nach $f$. maritimum)!!. Amrum: Norddorf (bei f. aristatum)!!. Sylt: Westerland (bei f. muticum)!!, MLorsum (bei $f$. aristatum)!!, Archsum (bei $f$. glaucum)!!, zwischen Archsum und Keitum (bei f. maritimum)!!. Amrum: Wittdün (Kuckuck und Polgar 1906!). Helgoland!!.

5. Deckspelzen stumpflich oder stumpf:

var. muticum Sickmann Enumeratio 13 (1836). - Häufige Form.

Dazu zählen :

f. arrense Reichenb. Icon. I. t. XX. fig. 1384 (1834), Blätter 4-8 mm breit, wenig rauh; Ährehen fünfbis achtblütig. - Häufigste Form der Rasse.

f. stenopleyllum A. u. Gr. Syn. Mitt.-Europ. Fl. II. 1. 646 (1901). - Blätter 2-3(-4) mm breit, stark rauh; Ährchen drei- bis fünfblütig. - So auf Sandboden (besonders Dünen) zerstreut, öfter in Übergängen nach f. maritimum.

- Deckspelzen stachelspitzig oder begrannt:

var. aristatum Sickmann a. a. O. 13 (1836). - Häufigste Form. Zerfällt in mehrere Abarten:

f. collimum A. u. Gr. a. a. O. 647 (1901). - Granne 2-3(-4) mm lang, plötzlich abgesetzt. -- So nicht selten. 
f. Taillantianum Döll a. a. 0. 129 (1857). - Granme bis reichlich halb so lang wie die Deckspelze, $4-6 \mathrm{~mm}$ lang, plötzlich abgesetzt. - Nicht selten.

f. sepium Döll a. a. O. 129 (185̃). - Granne etwa ron Deckspelzenlänge. - So im Gebiete bisher nur in Annäherungsformen.

f. Leersianm Reichenb. a. a. O. fig. 1388 (1834). Granne 3-8 mm lang, allmählich aus der Deckspelze verschmälert. - Sehr verbreitet.

f. subulatum Schreber in Schw. 1. Körte Fl. Erl. I. 143 (1804). - Deckspelze allmählich lang stachelspitzig ausgezogen. - Nicht selten.

6. Hüllspelzen deutlich gekielt; Deckspelzen undeutlich nervig:

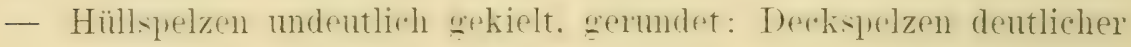
nervig:

var. glancum Döll Fl. v. Baden 130.(185̃7). - So im Gebiet zerstreut, auch auf Sylt und Röm.

Hierher gehören T. glancum Desfont. in Kunth Fl. r. Schlesw.-Holst. 785 (1887) und T. glaucum Dufour bei Hennings Naturw. Ver. Schlesw.-Holst. II. 1. 205 (1876) sowie T. pungens der Autoren des Gebiets z. T. (rgl. Prahl Krit. Fl. 264 [1890]). T. glaucum Desf. ist T. intermedium Host $=$ T. rigidum Schrader, welches Nolte aufzählt (Nor. Fl. Hols. 13 [1826]) und welches danach auch andere Autoren wie Laban und Borchmamn übernommen haben, das aber schon G. F. WW. Meyer für unser Gebiet ablehnt (Fl. hanor. excurs. 670 [1849]). Noltes Pflanzen gehören zu T. acutum.

7. Stengel $10-30 \mathrm{~cm}$ hoch, aus liegendem Grunde knickig aufsteigend; Ähre kurz, mit wenigen Ährchen; Ährchen wenig oder nicht über $1 \mathrm{~cm}$ lang, wenigblütig:

var. maritimum Koch u. Ziz. Catal. plant. Palat. 5 (1814). So am Strande auf Sandboden und an Deichen zerstreut, stellenweise nicht selten. Annäherungsformen finden sich auch binnenlands.

— Stengel $(0,5-) 1-1,5 \mathrm{~m}$ hoch, meistens aufrecht; Ähre verlängert, mit zahlreichen Ährchen: Ïhrchen l,is Q cm lang. tünf- bis achtblütig: var. litoreum A. u. Gr. a. a. O. ПI. 1. 650 (1901). - Am Strande zerstrent, stellenweise reichlich.

\section{Mißbildungen.}

f. m. ramosum not. f. - Ährchen an den Terzweigungen der Achse nicht simtlich einzeln, sondern besonders an Grunde der Ähre ötter zu zwei. seltener drei bis rier. - Lauenburg: Techin!! Hamburg: Hammerbrook (Laban 1866 !). Dampfmühle Wandsbek (A. Mohr 1904!). 


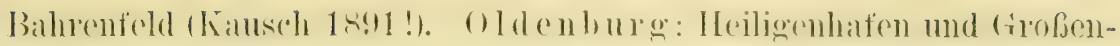
brode!!. Apeurade: Aubek!!. Helgoland (Hallier, Dalla-'Torre): am Kirchhof!!。

f. m. viviparum Friedrich Hb. - Ährchen in Laubsprosse auswachsend.

— Lïbeck: Wipperbrïcke (Friedrich 1893!).

f. m. bracteatum A. Christiansen nov. f. - Unterstes Ährehen mit laubigem

Tragblatt. - Kiel: Raisdorf (A. Christiansen!).

169. (s). 73). Triticum junceum L. Nant. alter. 329 (1771).

4. Grundachse kriechend, mit bis $3 \mathrm{~cm}$ langen Auslänfern. Stengel $(0,1-) 0,3-0,8 \mathrm{~m}$ hoch, aufrecht oder aufsteigend, glatt, kahl. Blattscheiden glatt, derb. Blattfläche 2-6 mm breit, öfter vom Rande eingerollt. glatt, oberseits an den Nerven je mit melneren Reihen von Hatren oder Höckern. Blatthäutchen sehr kurz. Ähre meistens $10-15(-20) \mathrm{cm}$ lang. mit sehr leicht zerbrechender, glatter Achse. Ährchen 1,6-3(-t) em limg. (drei- bis) fünf- bis zchnblütig. Hüllspelzen anm Rücken abgerundet, glatt, stumpt, untere 12-17, obere 14-18 mm lang. Deckspelzen bis $17 \mathrm{~mm}$ lang: stmmpf oder stachelspitzig. selten mit bis $3 \mathrm{~mm}$ langer, grannenartiger Spitze, undeutlich nervig. Blüte Juni bis August.

Auf Sandboden am Strande nicht selten, stellenweise häufig; auf den Nordseeinseln anf Föhr, Amrum, Sylt. Röm, Jordsand und Helgoland.

Zuerst erwähnt von Weber (Prim. Fl. Hols. Suppl. 5 [1787]).

\section{Formen.}

Zerfällt nach der Ährchengröße in zwei Formen: f. microstachyum Lange Haandb. Dansk. Fl. 2. Udg. 48 (1856). Ährehen bis 2,5 $(-3) \mathrm{cm}$ lang. - So verbreiteter.

Dazu als Unterform:

$f$. pygmaeum nor. f. - Stengel fein, bis $0,2 \mathrm{~m}$ hoch; Ähre kurz, wenigährig; Ährchen mit drei bis fünf Blüten. Sylt: List (Hansen 1846!), Keitum (Jaap 1897!).

f. macrostachyum Lange a. a. 0. 48 (18506). - Ährchen $3-4 \mathrm{~cm}$ lang. - So seltener.

G. F. W. Meyer treunt rou T. junceum (Chlor. Hanor. 610 [1836]) „b. hirtum. Haarigspelziger Binsenweizen" und erwähnt diese Form als in Lauenburg vorkommend Was darunter zu verstehen ist, vermag ich nicht anzugeben.

$\times($ ‥ $72 \times 73)$. Triticum repens $\times$ junceum $=$ T. pungens P'ers. Sin. I. 109 (1805).

Grundachse kriechend, auslüufertreibend. Stengel $0,3-0,8(-1) \mathrm{m}$ hoch, aufrecht oder aufsteigend, olatt, kahl. Blattscheiden glatt, wenig derb. 
Blattfliche 2-6 mm breit, oberseits und am Rande rauh, auf den Nerven mit einer oder meistens mehreren Reihen von Höckern oder Haaren. Blatthäutchen fast fehlend. Ähre 10-15 cm lang, mit nicht oder kaum brüchiger Achse. Ährehen $0,8-2,5 \mathrm{~cm}$ lang, drei- bis achtblütig. Hüllspelzen spitz oder stumpflich, sechs- bis nemmervig, untere 8-12, obere 10-13 mm lang. Deckspelzen stumpf oder spitz, selten begrannt, bis $14 \mathrm{~mm}$ lang. Pollen meistens fehlschlagend. Blüte Juni bis August.

Am Sandstrande der Nord- und Ostsee nicht selten oder zerstreut, an der Trave aufwärts bis Schlutup (Friedrich!), auf den Nordseeinseln stellenweise seltener, doch selbst auf Helgoland: Düne (Brody!).

Nolte war der erste Autor, der T. mungens aus unserer Flora nannte (Nov. Fl. Hols. 13 [1826]). Die Kreuzung ist eine sehr formenreiche; die Formen aber zeigen eine grobe Ungleichmäljigkeit in der Ausbildmg und Kombination der Merkmale, so daß ihre Gliederung schwer ist.

\section{Formen.}

1. Blätter auf jedem der etwas voneinander entfernten Nerren mit einer Haar- oder Höckerreihe; Hüllspelzen fast stets mit sechs Nerven: var. subrepens Marsson Fl. Neuvorp. Rügen 600 (1869). -

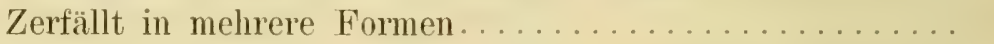

- Blätter auf jedem der genähert stehenden Nerven mit mehreren bis vielen Haar- oder Höckerreihen; Hüllspelzen mit (fünf bis) sieben bis nem Nerven:

var. subjunceum Marsson a. a. O. 600 (1869), - Zerfällt in zwei Rassen .

2. Deckspelzen stumpf oder stachelspitzig:

f. typicum nov. f. -- So häufig.

Hierher bei uns als Unterform:

f. pubescens Marsson a. a. 0.600 (1869). -- Ährenachse dicht behart. - Oldenburg: Heiligenhafen (Nolte 1825!)!!. Kiel: Friedrichsort (Nolte 1832!), Kronsort (A. Christiansen!). Schwansen: Schwansener Binnensee (A. Christiansen!). Angeln: Bokholmwik (Hansen!), Falshöft!!.

f. confertum Focke Abhandl. Nat. Ver. Bremen XII. 1. 181 (1905). - Ährchen gedrängt, einander zur Hälfte oder mehr deckend, erstes und zwölftes Ährchen im Ansatz nur $5 \mathrm{~cm}$ entfernt. - So im Gebiet bisher nicht festgestellt, in der Nähe z. B. bei Cuxhaven!!.

- Deckspelzen kurz begrannt:

f. aristatum Hornemann Oec. Plantel. 3. Udg. 1. 141 (1821). - Im Gebiet im Vorkommen unsicher. 
Die Form mit behaarter Ahrenachse nennt Reichenbach (Fl. Germ. excurs. 21 [1830]) von Warnemünde; darauf bezieht sich vielleicht Langmanns Notiz (Fl. Necklenb. 100 [1841]). Lange hat die Form abgebildet (Flora Danica t. 3005 [1883]). Nach der Größe der Ährchen tremnte Lange (Haandb. Dansk. Fl. 1. Udg. 46 [18,0]) f. mierostachyum (Ährchen bis 1,5 cm lang) und $f$. megastachym (Ährchen 2-2,5 cm lang), erstere häufiger.

3. Stengel $0,3-0,8 \mathrm{~m}$ hoch, ziemlich fein; Blattfläche in der Regel flach, am Rande rauh, auf den Nerven mit wenigen Haarreihen. Ährchen ziemlich kurz, mit zusammenschliefienden spelzen. Hüllspelzen spitzlich; Deckspelzen stumpflich oder nicht selten spitz, zuweilen stachelspitzig:

var. normale A. u. Gr. Syn. Mitt.-Europ. Fl. II. 1. 665 (1901).

- So zerstreut, mit allmählichen Übergängen nach var. subrepens und var. obtusinsculum.

Stengel 0,5-0,8(-1) m hoch, kräftig; Blattfläche derber, oft eingerollt, auf den Nerren mit zahlreichen Haarreihen. wie der Stengel oft auffällig blaugrün. Ährchen größer, mit zuletzt aufrecht abstehenden, spreizenden Spelzen. Hüllspelzen stumpf. Deckspelzen stumpf, zuweilen kurz stachelspitzig:

var. obtusiusculum A. u. Gr. a. a. O. II. 1. 666 (1901). - So seltener, besonders an der Ostsee.

Nords e e: Eiderstedt: Beenshallig (Nolte,Hb. Kopenhagen!), Tönning (v. Wasmer 1828 nach Prahl). Husum: zwischen Schobüll und Wobbenbüll (F. v. Müller 1845). Tondern: von Hoyer (Poulsen, Hb. Kopenhagen!; Hinrichsen!) über Emmerleff und Jerpstedt (Prahl 1879!) bis Ballum (Poulsen, Hb. Kopenhagen!). Föhr: Wyk (Schiötz! und Grönlund!, Hb. Kopenhagen). A mrum: Westküste verbreitet (J. Schmidt 1906!)!!, Ostküste mehrfach 1912!!. Sylt: zwischen Munkmarsch und Keitum (Jaap!). Röm: Lakolk (Jaap!).

Ostsee: Kiel (A. Christiansen!; eine Amnäherungsform). Fehmarn: Katharinenhof!! (weicht von den Exemplaren der Nordsee etwas ab).

Die Belege aus dem Kopenhagener Herbar haben sämtlich J. Lange, dem Autor des T. obtusiusculum, rorgelegen. Sie zeigen die charakteristischen Merkmale der Rasse z. T. weniger auffällig als sonst gesammelte Exemplare. T. litoreum Schumacher Enumeratio gehört nicht zu T. pungens, wie Kunth will (Fl. Nordfries. Inseln 152 [1895]).

170. (adv. 84). Triticum cristatum Schreber (tram. II. t. 2:3 (176!). 4. Grundachse dichtrasig. Stengel anfrecht, seltener aufsteigend, 
0,2-0,6 m hoch, kahl, glatt oder unter der Ähre schwach rauh. Blattscheiden glatt, kahl. Blattfläche 2-4(-5) mm breit, unterseits glatt, kathl. oberseits kuzhamig. zumeilen eingerollt. Blatthäutchen fist fehlend. Ähre : -6 cm lang. mit dichtgedrängten, anfrecht abstehenden ت̈hrchen. Ährchen 6--10(-15) mm lang, drei- bis acht- (bis zehn-)blïtig. Hüllspelzen mit starkem Mittelnerv jederseits mit oder ohne zwei kurze. schwache Nervell. $3-4 \mathrm{~mm}$ lang. mit bis $3 \mathrm{~mm}$ langer (iramne. am Xittelnerv gewimpert, düm hautrandig. Deckspelzen bis $6 \mathrm{~mm}$ lang; undentlich nerrig; mit bis $3 \mathrm{~mm}$ langer (tranne. kahl oder kurzharig. Blüte Juni bis Juli.

Auf Schutt bei Hamburg selten.

Hamburg: Kinhwärder (Laban $18 \times 6$ !). Wandsbek (.T. Sichmidt 1889!, 1862!!. Damptmühle Wimdsbek (.J. schmidt 1895 !. Zimpel 1896!, 1897!). Winterhude (Laban, Berichte Deutsch. Bot. Ges. T. CIV. [1887]), am Diebsteich in Altona (Laban a. a. O.. . . schmidt 1886!). Langenfelde (Laban 1891!).

\section{1. (adv. 85). Triticum prostratum L. fil. suppl. $11+(1781)$.}

¿. Stengel zu wenigen bis rielen, aufrecht oder aufsteigend, meistens $5-10(-20) \mathrm{cm}$ hoch, kahl oder unter Knoten und Rispe kurzhaarig. Blattocheiden kurz. die oberen etwas aufgeblasen. kahl oder kurzhaarig. Blattfläche kurz. 1-3 mm breit. beiderseits zerstrent kurz behaart. Blatthäutchen sehr kurz. Ähre kurz. rundlich bis eiförnig: mit dichtgedrängten Ährchen. Ährchen $5-7$ mm lang: (drei- bis) rier- bis sechsblïtig. Hüllspelzen 3,5-4 mm lang; 1-2 mm lang begrannt. jederseits des Mittelnerren mit gelblichweibem. dickem Knorpelstreit. kahl, hautrandig. Deckspelzen bis t.5 mm lang, mit $1 \mathrm{~mm}$ langer. gramenartiger spitze, kahl, undentlich nervig. Blïte Mai und Juni.

Auf Schutt bei Hamburg selten.

Hamburg: Dampfmühle Wandsuek (Zimpel 18sit!; Deutsch. Bot. Monatsschr. XVI. 115 [1898]).

172. (adr. 86 ). Triticum orientale II. Bieb. Fl. Taur.-Canc. I. 86 (1808). $\odot$. Stengel tnd Blätter wie bei voriger Art. Ähre $1-3 \mathrm{~cm}$ lang; hopfig bis kurz zỵlindrisch, mit abstehenden Ïlnchen. Ährchen bis $7 \mathrm{~mm}$. mit (iramnen bis 1.1 ('m lang. drei- bis riel- (bis fünf-)blütig. Hüllspelzen bis $7 \mathrm{~mm}$ lang, mit 3-4 mm langer Granne, so lang wie das ährchen oder wenig kü̈zer. behaart, weniger knorpelig rerdickt. Deckspelzen aus breitem (irunde allmählich in die bis 3 mm lange Grame rerschmälert, dicht weißhaarig bis (seltener') kahl. Blüte Juni bis August. 
Auf Schutt bei Hamburg selten.

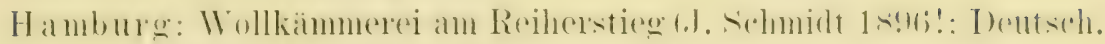
Bot. Monatsschr. XVI. 115 [1898]; auch mit kahlen Deckspelzen).

Vom Trpus weicht ab:

\section{Formen.}

f. lasianthum Boissier Fl. Orient. V. 668 (1884). - Spelzen dicht wollig behaart. -- So mit der Art (J. Schmidt!, a. a. O.).

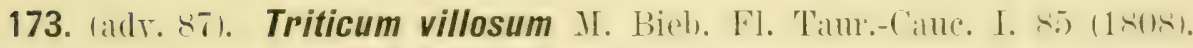

$\odot$. Stengel zu wenigen bis vielen, aufrecht oder meistens aufsteigend, 0.2-0.6 m hoch. glatt. Blattscheiden glatt, kahl wder die unteren

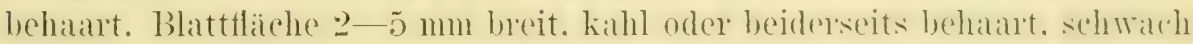
rauh. Blatthäutchen kurz, gezilnelt-zerrissen. Thre 3-bit-101 c'ml lang. mit gedrängten. aufrecht abstehenden Ährchen. Ährchen 1.2-1.5r-1.81 ('m lang, zwei- bis rier- (bis tünt-blütig. Hüllspelzen zweikielig. 5- ti mm lang, an beiden Kielen gewimpert rabhhanig. mit abgesetzter. :3 - + 'm langer (iramme. Deckspelzen bis $1,2 \mathrm{~mm}$ lang, kithl oder selten tein rauh. mit 3-4(-5) cm langer Granne, am Grunde der Granne behaart, undeutlich fünfnervig. Blüte Juni bis August.

Auf Schutt selten.

Stormarn: Reinbek (C. Timm, Laban 1884!). Hamburg: bei IFandsbek anf dem Königsland (C. T'imm 1891!) und bei der Damptmühlte (Tal) 1892!). Winterhude (Laban 1885!. 1891!) bei Altona am Diebsteich IC. Timm usw. 1886 !) und am Mennonitenkirehhof IC. Timm. W. Hansen 18st!.

Vom Typus weicht ab:

\section{Formen.}

f. brachystachyn nov. f. - Ähre eiförmig, mit nur drei bis vier Ährchen. - Hamburg: Dampfmühle Wandsbek (Zimpel 1894!). Vielleicht Mißbildung.

174. (adr. 84). Triticum sativum Lam. Encrel. II. 55t 117ה(3).

$\odot$ oder $\odot$. Stengel einzeli oder zu mehreren, aufrecht, $(0,3-)$ 0.6-1.t m hoch. derb. glatt. kahl. Blattscheiden kihl oder kumhaarig.

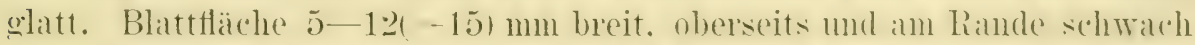
rauh. muterseits glatt. ofter oberseits kurzharig. Blatthäutehen $2--4 m m$ lang; breit, gezähnelt. Ähre 4-10 cm lang; selten länger', mit zäher oder brïchiger Achse. Ährchen 10-15 mm lang, zwei- bis fünfblütig; doch nur mit einer bis drei Früchten. Hüllspelzen s-10 oder mit quannenartiger Spitze bis $15 \mathrm{~mm}$ lang. kahl oder seltener behant. oft mit jeder- 
saits einem seitenzahne. Ineckselelen bis 10 num lang. begramut oder gramenlus. kahl oder beonders nberwarts und am Pande behant. undentlich nervig. Torspelze ron der Länge der Derhspelze. Blüte Juni und Juli. auf Schuttland bis Oktober.

Nicht selten gebaut und oft rerwildert.

\section{Formen.}

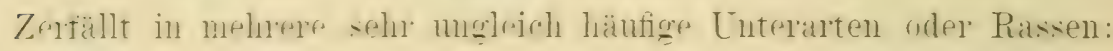

a. T. spelta L. Spec. plant. ed. 1. 86 (1753). - Ährchenachse bei der

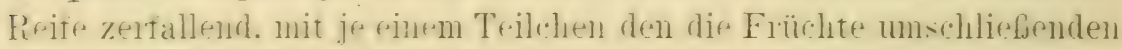
spelzen anlattend. Älnelen mit zwei Früchten. beidenseits gewölbt. ihne Hüll-nelzen mit breit aldgeschnittenem oberem Ende. mit geradem Kiel.

Ob bei uns gebaut?; hin und wieder, doch selten, auf Schutt rerschleppt.

b. T. vulgare Till. Hist. pl. Dauph. II. 153 (1787). - Ährenachse zäh; Frürhte für sich allsallend. Hüllswelzen nur oberwäits deutlich gekielt, unterwärts nicht gekielt.

So nicht selten gebant und rerwildert oder verschleppt.

Dazu verschiedene Formen:

f. albidum Alef. Landwirtsch. Fl. 329 (1866). - Dechspelzen grannenlos, kahl. - Häufigste Form.

f. celutinum Ascherson Fl. Brandenb. I. 870 (186t). - Deckspelzen grannenlos, dicht kurz und weich behaart. - Sehr zerstreut, oft wenig.

f. submuticum Ascherson a. a. O. I. 870 (1864). - Deckspelzen mit kurzer, 1-4 cm langer Granne, kahl. - Zerstreut.

c. T. compactum Host Gram. Austr. IV. 5. t. 7 (1809). - Wie T. vulgare, aber nicht mit schlinher. rielmals die Breite an Länge übertreffender ت̈hre sondern mit kurzer ähre die zwei- bis rier- bjis tünt-mal so lang wie breit ist.

So selten gebaut; ob rerschleppt?

d. T. turgidum L. a. a. 0. 1. 86 (1753). - Ährenachse zäh; Früchte rrei ausiallend: Hüllspelzen rom Grunde deutlich gehielt. Deckspelzen mit oft rerlängerter Granne.

Selten gebaut (Sonder, Fl. Hamb. 73; Prahl, Krit. Fl. 263). zuweilen reschlfunt. \& Hamburg: Kuhwärder (C. Timm 1sss!). Hamm (Kansch)!!, am Diebsteich in Altona (C. Timm, IT. Hansen 1885 !). Bahrenfeld 1900!!

Dazu:

f. Znccale Alef. a. a. O. 326 (1866). - Hüll- und Deckspelzen dicht

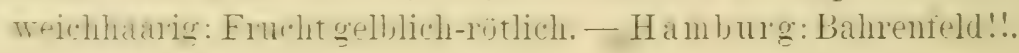


175. (adr. 89). Triticum ovatum Godr. u. Gren. Fl. France HI. 601 (1856) el'w.

$\odot$. Stengel zu mehreren bis vielen, aufsteigend, $0,2-0,4 \mathrm{~m}$ hoch, glatt, kahl. Blattseheiden kahl oder oberwärts am liande zerstrent gewimpert. Blattliathe $2-3(-4) \mathrm{mm}$ breit, kahl oder (besonders oberseits) behaart, am Ramde schwald rauh. Blatthäutchen ku\%. Ähre (2-); -5 cm lang, wenigährig. Ährehen bis 1,5 em lang, drei- bis vierblïtig. untere fruchthar, obere (oft nur eins) mufruchthar. IIüllspelzen der fruchtbaren Ährehen bis \& mm lang. sehr dert), tünf- bis mehrnervie. oben breit abgeschnitten, mit zwei bis drei (iramen und (oder) Zä̈hnen, am Rï̈rken kurz behaart. Deckspelzen bis $11 \mathrm{~mm}$ lang. unterwärts gelblich. glänzent. fast mgenervt, oberwäts mit dentlich hervortretenden. kurz ranhhaarigen Nerven, mit zwei bis drei kurzen, höchstens 2 cm langen Grannen. Unfruchtbare Ährehen viel kleiner, nur ihre trammen denen der fruchtbaren etwa gleich. Blüte Mai bis August.

Auf Schutt bei Hamburg selten.

H a mburg: in Wandsbek bei der Helbingschen Branerei (.T. Sichmidt 1892 !) und bei der Dampfmïhle (I. Schmidt!, Zimpel! 1894), Bahrenfeld (Zimpel 1901)!!

\section{Formen.}

Bei uns wurden zwei Rassen beobachtet:

1. var. triaristatum A. 11. Gr. Syn. Mitt.-Europ. Fl. II. 1. 705 (1902). -- Ähre mit drei (bis vier) fruchtbaren und einem bis zwei unfruchtbaren Ährchen; Hüllspelzen in der Regel dreigrammig, zuweilen nu' zweigrannig.

So am ersten und dritten Standorte. (J. Schmidt Deutsche Bot. Monatsschr. XIII. 111 [1895]).

- var. biunciale A. u. Gr. a. a. O. II. 1. 706 (1902). - Ähre mit zwei Ährehen, kurz; Hüllspelzen am unteren Ährchen mit zwei, am oberen mit drei Grannen.

So am zweiten Standorte! (Prahl Fl. v. Schlesw.-Holst. 2. Aufl. 41 [1900]).

176. (adv. 9()). Triticum triunciale (iodr. 11. (iren. Fl. France III. 6(1)2 (185)(6).

$\odot$. Stengel zu mehreren, knickig aufsteigend. (1..2-0),4 m hoch. glatt. Blattscheiden in der Regel kahl. Blattliache 2-:3 mm breit, am Ramde rauh, oberwärts oft behaart. Blatthäutchen kurz. Ähre $4-6 \mathrm{~cm}$ lang; dicht. mit drei bis fünf fruchtbaren und zwei bis drei unfruchtbaren Ährehen. Ährehen bis 1,2 cm lang. zwei- bis drei- (bis vier-blütig. Hüllspelzen und Deckispelzen denen der vorigen Art ähnlich. arstere an den oberen Ährehen mit sehr (bis etwa $8 \mathrm{~cm}$ ) verlängerten, an den unteren 
Ährehen mit kürzeren $(2-4 \mathrm{~cm})$ Grannen, letztere mit zwei bis drei Zïhnen oder Grannen. an den untersten Ährehen oft nur gezähnt oder sehr kunz begrannt (bis s mm). an den oberen Ährchen länger begrannt. Blüte IIai bis August.

Auf Schutt bei Hamburg selten.

Hamlurg: bei der Inampiminhe Wrandshek (.T. Sechnidt usw. 1894!), am Diebsteich in Altona (C. Timm, Laban usw. 1885!; Prahl Krit. Fl. $268[1890 \mid)$.

177. (adv. 91). Triticum cylindricum ('eatt. Pass. (ril). Comp. 86 (1869).

$\odot$. Stengel zu mehreren, aus oft geknickt niederliegendem Grunde allfsteigend. $0.2-0.6 \mathrm{~m}$ hords. glatt. Blattseheiden am stengelgrunde meistens: zerstreut behialt. im oberen Teile des stongels in der Regel kahl. Blattflärhe o--6 mm breit, schwach rauh. am Ramle stark rauh. heiderseits zerstrent kurzhatarg. Blatthäutchen kur. Ähre lis $15 \mathrm{~cm}$ lang. schmal, ein bis zwei Ïhrehen am cirumbe verkimmert, 5-6 $\mathrm{mm}$ lang. vier bis nem Ährehen fruehthar. ein his zwei Ährehen an der Spitze mfruchthar. lis $15 \mathrm{~mm}$ lang. Fruchtbare ährehen his 15 mm lang, Izweihis) vierblütig. ihne Hüllspel\%en derl). viehnervig, an Rücken stark rauh, amm oberen Ende ungleich zweizähnig oder ans einem Zahne kurz be(rannt (bis etwa 10 mmo. ihre Deckspelzen bis 12 $11 m$ lang, oberwärts drutlich nerig mol ranh. oben zweizänig. Endährchen an den Hüllspelzen und meistens anch den untersten Deckspelzen mit bis $7 \mathrm{~cm}$ langer, gerader, sehr rauher Granne. Bliite Mai bis September.

Auf Schutt bei Hamburg.

Hamlurg: bei der Wollkämmerei am Reiherstieg (.J. Schmidt 1895̃!), mehrfach im Hammerlorork (Bruns!. C. Timm Ber. Dentsch. Bot. Ges. T.

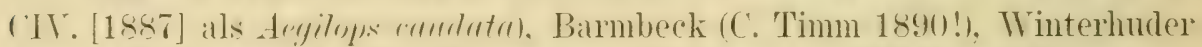
Bruch (.J. Schmidt 18 !) !), Irinterhude (.J. Schmidt 1888!), bei Altona am Inebsteirh (Eriehsen 1887!), bei der Holstenbanterei (Zimuel 1891!), am Temnonitenkirchlhof (C'. Timmm und bei Bahrenfeld!!, bei Wandsbek auf dem Königsand (IV. T'imm 1890!). bei Helbings Branerei (Zimpel 1894!) und bei der Dampfimühle (Zimpel 1894!)!!.

178. (arly. (12). Triticum speltoides (fodl. Fl. Massil. 434 [48] (1857). $\odot$. Stengel zu mehreren, aufrecht oder aufsteigend, $0,3-0,8 \mathrm{~m}$ lorel. glatt. Blattscheiden kiahl oder die unteren zerstreut kurz behaart. Bhittliache 2- 4 mm breit. rauh, beiderseits nud am Rande kurzhaarig. [Blatthäutchen sehr kurz, gräthnelt. ت̈hre $7--9$ cm lang. schmal. Unteres Ïhrehen rerkïmmert, l,is 5 mm lang. Fruchthare Ährehen bis $11 \mathrm{~mm}$ 


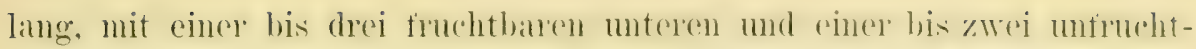

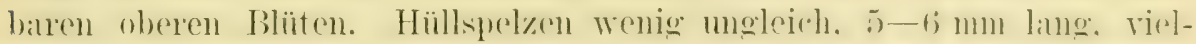

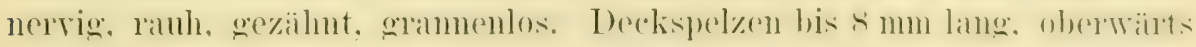
deutlich nervig, rauh, mit $5-9(-10) \mathrm{cm}$ langer Granne oder (an den oberen Blüten) gramnenlos. Blüte August bis Oktober.

Auf Schutt bei Hamburg selten.

H a mburg: bei der Wollkämmerei am Reiherstieg (Zimpel 1895!, J. Schmidt 1896!, 1897!) (Aegilops bicomis Boiss. z. 'T., Pieper Dentsch. Bot. Monatsschr. XVI. 115 [1898], nicht Jaubert und Spach; rergl. Ascherson in Magyar Bot. Lapok I. 1. 6-12 [1902]).

Bei uns nul

\section{Formen.}

f. Tigusticum A. u. Gr. a. a. O. I. 1. 11 (1902). - Deckspelzen aller Ährchen gleichartig begrannt. - So am angeführten Standorte.

Triticum vulgare $\times$ cylindricum ist aus dem Grebicte bisher nicht bekamnt. Nach Laban (Fl. r. Hamb. 4. Aufl. 190 [1887]) fand C. Timm am Diebsteich in Altona eine Zwischenform von $T$. vulgare und Aegilops caudata (bei ihm = Ae. cylindrica). Es wird sich wohl um eine Kümmerform von. $T$. vulgare gehandelt haben; derartige Formen erimnern zuweilen im Habitus auffällig an bestimmte Arten der Sektion Aegilops.

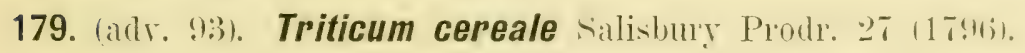

$\odot$ und $\odot$. Stengel zu mehreren, meistens anfrecht, $0,5-1,5(-2) m$

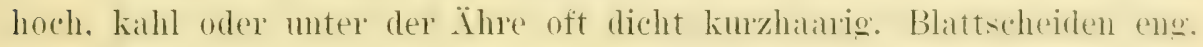
glatt, kahl. Blattfäche $(4-) 8-1,5(-2) \mathrm{cm}$ breit, unterseits glatt, oberseits mud an Rande rauh. Blatthäutchen bis -) mu limg. am Rande fein zerrissen. Ähre $(5-) 8-12(-20) \mathrm{cm}$ lang, dicht, mit zäher Achse. Ährchen bis $16 \mathrm{~mm}$ lang, zwei- (bis drei-)blütig. Hüllspelzen lineal, einnerrig; gekielt, häutig. spitz. Deckspelzen bis 15 mm lang. lanzettlivh. dreinerrig. moleichhältig gekielt, an Kiel borstleh gewimpert. allmählieh in dir

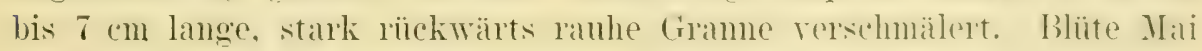
bis Juni, einzeln bis Oktober.

Häufig gebant und oft selbst aut Helgoland) vermildert. ant sichutland rerschleppt.

Die Art zerfällt:

\section{Formen.}

1. Ahrchen zweibliitig:

f. vulgare Körnicke in Körm. u. Wern. Handb. Getr.-Ban I. 127 (1885). - Stengel unter del Ähre behaart. - So lıänfig:

Ährchen sämtlich (oder teilweise) dreiblütig:

f. triflorum Ascherson Fl. Brandenb. I. 871 (186t). — So selten. 
wohl öfter übersehen. - Ha mburg: Georgswärder (Zimpel!), Uhlenhorst (C. 'Timm 1872!), Eppendorf (Erichsen 1890!), Langenfelde (A. Junge!), Bahrenfeld (Zimpel!). Lübeck: Herrenwiek::

\section{Mißbildungen.}

f. m. monstrosum Körnicke a. a. O. I. 128 (1885). - Ähre geteilt oder verzweigt (in sehr ungleicher Ausbildung). - So ziemlich selten und meistens wenig.

\section{Gattung.}

\section{Hordeum.}

L. Gen. plant. ed. 5.37 (1754).

1. Ährchen drei- (bis vier-)blütig; alle Spelzen gramenlos.......H. arenarium.

- Ährchen ein- (bis zwei-)blütig; Spelzen wenigstens z. T. begrannt......... 2

2. Ährchen einzeln oder zu zweien .......................... 3

- Ährchen zu dreien...................................... 4

3. Pflanze 4; Deckspelze etwa $8 \mathrm{~mm}$ lang, mit bis $2 \mathrm{~cm}$ langer Granne.

H. europaeum.

- Pflanze $\odot$; Deckspelze 7-8 $\mathrm{mm}$ lang, mit über $6 \mathrm{~cm}$ langer, stark rauher

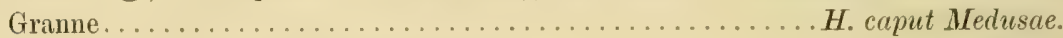

4. Alle drei Ährchen mit Zwitterblüten.................. polystichum.

- Die beiden Seitenährchen nicht nit Zwitterblüten............... 5

5. Seitenährchen mit je einer männlichen Blüte $\ldots \ldots \ldots \ldots \ldots \ldots \ldots \ldots \ldots$

- Seitenährchen ohne Blüte (Blüte verkümmert) $\ldots \ldots \ldots \ldots \ldots \ldots \ldots \ldots \ldots \ldots$

6. Deckspelzen der Seitenährchen unbegrannt.................... 7

- Deckspelzen auch der Seitenährchen begrannt .................. 8

7. Ährenachse brüchig; Hüllspelzen dicht kurz behaart ........H. spontaneum.

- Ährenachse zäh; Hüllspelzen kahl oder zerstreut behaart ........H. distichon.

8. Hüllspelzen des Mittelährchens beiderseits gewimpert.......... m. murinum.

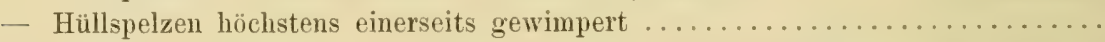

9. Pflanze $\odot$; Stengel bis zur Rispe beblättert; innere Hüllspelzen der Seitenährchen mit schmallanzettlichem Grunde............... maritimum.

- Pflanze 4; Stengel oberwïrts blattlos; innere Hüllspelzen vom Grunde grammen-

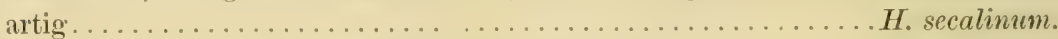

10. Deckspelze mit ihrer starren Grame 9-13 mm lang ......... compressum.

- Deckspelze mit ihrer feinen Granne $4-6,5 \mathrm{~cm} \operatorname{lang} . . . \ldots \ldots . . . . . . j u b a t u m$.

180. (adv. 94). Hordeum spontaneum K. Koch Limnaea XXI. 300. 430 (1848).

$\odot$. Stengel zu mehreren, aufrecht oder aufsteigend, 0,5-1,2 $\mathrm{m}$ hoch, glatt, kahl. Blattscheiden kahl, glatt. Blattfläche 4-10 mm breit, unterseits glatt oder kaum rauh, oberseits stark rauh, am Rande sehr rauh. Blattläutchen etwa $2 \mathrm{~mm}$ ling, breit, stumpf. Ähre $4-11 \mathrm{~cm}$ lang, dicht, mit brüchiger, bei der Reife zerfallender Achse. Ährchen bis $11 \mathrm{~mm}$ 
lang, zu dreien, das mittlere Ährehen sitzend, cinblütig, die beiden seitlichen mit kurzem, behatatem Stiel. Hüllspelzen lineal, spitz grannenartig ansgezogen, an den seitlichen ت̈hrehen bis $11 \mathrm{~mm}$, am mittleren bis $13 \mathrm{~mm}$ lang. unterwärts dicht behaart, oberwärts ranh. Deckspelzen kahl, schmallanzettlich, an den seitenährehen efwal 10 mm lang, grammenlos, am Mittelährchen etwa $11 \mathrm{~mm}$ lang, mit bis über $10 \mathrm{~cm}$ langer Gramne. Blüte Juni und Juli.

Auf Schutt selten verschleppt.

Hamburg: bei der Wollkämmerei am Reiherstieg IZimpel 1896!, 1897!; Dentsch. Bot. Monatsschr. XVI. 115 [1898). (Für Mittelemropa bei Montpellier durch Thellung festgestellt.)

181. (adv. 95). Hordeum distichon L. Spec. plant. ed. 1. 85 (175:3).

$\odot$. Stengel zu mehreren, in der Regel aufrecht, $0,6-1,2 \mathrm{~m}$ hoch, glatt. Blattscheiden glatt, kahl. Blattfläche $\check{0}-15 \mathrm{~mm}$ breit, raul. Blatthäutchen kurz. Ähre $6-10(-15) \mathrm{cm}$ lang, dicht, mit zäher Achse, bei der Reife nicht zerfallend. Ährchen sehr moleich entwickelt; Mittelährchen größer, fruchtbar; Seitenährchen kleiner, unfruchtbar, dem Mittelährchen angedrückt, daher Ähre anscheinend zweizeilig. Hüllspelzen lineal, kahl oder seltener sehr spärlich behaart, höchstens so lang wie die Deckipelze. Deckspelze der Seitenährchen stumpf oder stumpflich. grammenlos, des Vittelährchens breiter, mit bis $20 \mathrm{~cm}$ langer, rorwärts rawher Grame. Frucht beim Ausfallen von der Deckspelze umhüllt. Blüte Juni und Juli, öfter spärlich bis Oktober.

Oft gebaut und verwildert, nicht selten auf Schutt verschleppt.

Auf die Formen dieser wie der folgenden Art sowie der angebauten TriticumArten ist noch zu achten.

182. (adv. 96). Hordeum polystichum Haller Gen. spec. nor. ('om. Goett. 6 (1776).

Der vorigen Art sehr ähnlich, durch die Ausbildung der Seitenährchen unterschieden. Seitenährehen abstehend. neben den beiden Reihen der Mittelährehen zwei oder vier Reihen der seitenährehen bildend. sämtlich fruchtbar.

Weniger oft gebaut, nicht selten verwildert und verschleppt.

\section{Formen.}

Bei uns zwei Rassen:

1. Seitenährehen beider Reihen einer Ährenseite abwechsehnd zwischen einandergreifend, die Ährchen daher in vier Reihen: var. vulgare Döll Rhein. Fl. 67 (1843), - So häufiger. 
- Seitenährchen getrennt, die Ährchen daher in sechs Reihen:

var. hexastichon Döll a. a. O. 66 (1843). - So seltener; verschleppt und selten gebaut.

183. (sp. 74). Hordeum secalinum Schreber Spicileg. Fl. Lips. 148 (1771).

4. Grundachse dichtrasig. Stengel aufrecht oder aufsteigend, glatt, kahl, 0,3-0,8 m hoch. Untere Blattscheiden zerstreut abstehend behaart. eng, obere kahl, oft etwas aufgeblasen. Blattfläche $2-3(-5) \mathrm{mm}$ breit. oberseits und am Rande-schwach rauh, unterseits glatt. Blatthäntehen sehr kurz. Ähre $2-5 \mathrm{~cm}$ lang, dicht, mit bei der Reife zerbrechlicher Achse. Ährchen ungleich; Mittelährchen fruchthar, 8-9 mm lang, mit grannenartig schmalen, bis $12 \mathrm{~mm}$ langen Hüllspelzen und ron diesen überragter, bis $8,5 \mathrm{~mm}$ langer, lanzettlicher, bis $11 \mathrm{~mm}$ lang begrannter Deckspelze; seitenährehen gestielt, mit mämlicher Blüte, 6 mm linng. mit gramnenartig schmalen, bis $14 \mathrm{~mm}$ langen Hüllspelzen und nur 5 mm langer. 3-7 mm lang begrannter Deckspelze. Blüte Mai bis Juli.

Auf Wiesen und Weiden, an Uferm; an der Ostsee von Neustadt bis Hadersleben nicht selten bis zerstreut (ziemlich entfernt rom Strande z. B. Fehmarn: am Wege zwischen Burg und Niendorf!!), doch auf Alsen nur bei Sonderburg (Matthiessen), an der Tordsee hänfig bis zerstrent, auf den Tnseln auf Röm: 'T'oftum (.J. Schnidt) und Kongsmark!!, Sylt (Sehiötz): am (Strande nicht selten (O)stermeyer)!!, A mlum (Nolte 1825!), Föhr (Schiötz). Hallig Oland Nolte 1825!) und Helgoland: Oberland (Hallier, Brody!), an der Eider bis oberhalb Friedrichstadt: Delver Deich (Bargum 1808!), an der Elbe bis Brmsbüttel häufig!!. oberhalb nicht selten, aber oft spärlich, bis Hamburg. weiter aufwärts nur Lanenburg: in der Besenhorst (Sonder, Borchmann!) (am Mittellaufe noch in Branderiburg [Landeskunde p. 145]).

Außerdem auf Schutt bei Hamburg: Kl. Grasbrook (Laban!), Eppendorf (.J. Schmidt 1890!), Winterhuder Bruch (J. Schmidt und Zimpel 1890!), in Altona bei der Holstenbranerei (Zimpel 1891!) und beim Kirchhof (A. Junge 1890!).

Nolte nemnt ${ }^{\prime} H$. secalinum als $H$. pratense Huds. (Nov. Fl. Hols. 14 [1826]); in dex Flora Danica wird die Art bereits früher erwähnt (t. 630 [1775]). Wenn eine Hamburger Arbeit als Autor für H. pratense „L." setzt (Ver. Naturw. Unterh. VII. 66 [1894]), so liegt ein Irrtum vor. Der Linnésche Name H. nodosum (z. B. verwandt Progr. Unterrichtsanst. St. Johamn. Hamb. 32 [1890]) ist in seiner Beziehung auf H. secalinum ganz zweifelhaft. Bei der Wollkämmerei, beim Hellbrook und für die Uhlenhorst erwähntes H. secalinum gehört nicht zu dieser Art!.

\section{Formen.}

Vom Typus weicht ab:

f. marinum Koch Syn. Fl. Germ. et Helv. ed. 2. 956 (1844). - 
Deckspelzen der seitenährehen und of anch der Mittelährehen mehr oder weniger dicht behaart. - So an Nord- und Ostsee zerstreut. stellenweise reichlich.

184. (*1). 75). Hordeum maritimum Withering Bot. Arrang. 172 (1776).

$\odot$. Stengel in der Regel zahlreich, aus liegendem Grunde aufsteigend, $0.1-0,3 \mathrm{~m}$ hoch, glatt, kahl. Blattscheiden am stengelgrunde beharrt, obere kahl, etwas autgeblasen. Blattfläche 2-3 mm breit, oft etwas eingerollt, glatt. kahl oder dicht behart. Blatthäutchen fast fehlend. Ähre $2-3(-5) \mathrm{cm}$ lang, dicht, mit zerbrechlicher Achse. Ährehen mgleich; Nittelährchen mit zwei gramnenartigen, 1,5-0..5) (cm langen Hüllspelzen und mit bis $7 \mathrm{~mm}$ langer, lanzettlicher. 1.4-2.2 $\mathrm{cm}$ lang begramnter Deckspelze; Seitenährehen mit einer gramenartigen und einer am Grunde $0.5-1 \mathrm{~mm}$ breiten. bis 2 cm langen Hüllspelze und mit 3-4 mm langer, kurz begrannter Deckspelze. Blüte Mai bis Juli.

Auf Salzwiesen und an Deichen nur im Nordseegebiet ron Dithmarschen bis Pellworm und Dagebüll. Dithmarschen (Nolte Nor. Fl. Hols. 14 [1826]. doch ohne Belege und nenerdings nicht getunden). Eiderstedt (F. v. Müller!): Ording (Hb. Kopenhagen 1851!) Husum (Nolte 1825!): rom Finkhause bis Dreisprung, bei Brorsum (F. r. Mü̈ller?). Simonsberger Kong (r. Fischer-Benzon), Tuliane Marie-Koog (Nolte nach Lange), Dagebüll (Tahl 1797!. Hb. Kopenhagen!; Bargum 1s(13!). Nordstrand (Nolte 1825!), Pellworm (Nolte 1825!).

Ferner auf Schutt bei Ha mburg: bei der Wollkämmerei am Reiherstieg (Laban 1896!, J. Schmidt 1898!), Georoswärder (Zimpel 1894!). Dampfmühle Wandsbek (Zimpel und J. Schmidt 1894!).

Nolte ist nicht der erste, welcher $H$. maritimum aus dem Gebiete erwähnt; bereits durch Mößler (Handh). Gewächsk, 101 [1815), ferner durch Homemamn (Flora Danica t. 1632 [1819] wie Oec. Plantel. 3. Udg. 1. 139 [1821]) wird das Gras aufgeführt. Die Erwähnungen beziehen sich z. T. nicht wirklich auf $H$. maritimum, sondern auf $H$. secalimum. so die Hornemamnsche ron Heiligenhafen (rgl. Prahl hrit. Fl. 261 [1890]) Auf Sylt und Föhr wurde die Art bis jetzt nicht gefunden (Knuth Fl. Nordfries. Ins. 151 [1895] und Bot. Wand. Sylt. 115 [1890] nach Prahl Bot. Ter. Brandenb. XITII. 24 [1876]).

\section{Formen.}

Vom Typus weicht ab:

var. Gussoneanum Richter Plant. Europ. I. 131 (1890). - Hüllspelzen der Seitenährchen grannenartig. anch im unteren Teile gleich oder sehr wenig verschieden.

H a mburg: Georgswärder (Zimpel 1894!). Dampfmühle Wrandsbek (J. Schmidt 1894!). 
Die Pflanzen sind durch allmähliche Übergänge in der Hüllspelzenbreite mit H. maritimum verbunden. So breit wie an Exemplaren ron der Nordseeküste ist die breitere Hüllspelze der Seitenährchen an dem $H$. maritimu der Hamburger Schuttplätze nie.

185. (qs]). 14). Hordeum murinum L. spec. plant. ed. 1. 85 (1753).

$\odot$ und $\odot$. . Stengel zu mehreren, aufrecht oder aufsteigend, selten am Grunde lang niederliegend, 0.2-0.6 m hoch, glatt, glänzend. Blattscheiden kahl oder die unteren schwach behaart, glatt, die oberen etwas aufgetrieben. Blattfäche $2-6 \mathrm{~mm}$ breit, schwach rauh, kahl oder zerstreut kurz behaart. Blatthäutchen sehr kurz. Ähre 5-12 cm lang, dicht. Ährchen 10-11 mm lang; Xittelährchen mit schmallanzettlichen, beiderseits gewimperten, in eine etwa $2 \mathrm{~cm}$ lange Grame ausgezogenen Hüllspelzen und mit lanzettlicher, bis $4.5 \mathrm{~cm}$ lang begrannter Deckspelze; Seitenährchen mit einer (äußeren) grannenartigen und einer (inneren) schmal linealeu und kurz gewimperten. mit Gramne bis $3 \mathrm{~cm}$ langen Hüllspelze und mit bis $4 \mathrm{~cm}$ lang begrannter, lanzettlicher, kahler oder schwach rauher Deckspelze. Blüte Juni bis Oktober.

An Wegrändern. Zäunen, Hecken, auf Gras- und Schuttland, besonders in Ortschaften; im östlichen und mittleren Gebiet nicht selten bis zerstreut, nordwärts seltener bis Sonderburg (Petit) häufig (H. Petersen), im Testen nur in Eiderstedt und bei Husum (r. Fischer-Benzon) sowie bei Tondern (H. Schmidt 1910!), ferner auf den Nordseeinseln Fordstrand (r. Fischer-Benzon), Pellworm und Hooge (Kinuth) und Föhr (Nolte 1825!): Wyk (schiötz 1858!). doch nicht auf Helgoland.

Zuerst genannt von Weber (Prim. Fl. Hols. 11 [1780]).

\section{Formen.}

Als Rasse (oder Unterart?) weicht ab:

var. leporinum Richter Plant. Europ. I. 130 (1890). - Hüllspelzen der Seitenährchen stark gewimpert (die inmere beiderseits, die äußere nach innen), beide verbreitert, etwa $1 \mathrm{~mm}$ breit.

Hamburg: verschleppt bei der Wollkämmerei am Reiherstieg (J. Schmidt 1896!, Zimpel 1897!).

\section{6. (adv, (17). Hordeum juhatum L. Spec. plant. ed. 1. 85 (1753).}

¿. Stengel zu mehreren, aufrecht oder kurz aufsteigend, 0,3-0.6 m hoch. glatt. kahl. Blattscheiden glatt. kahl oder die muteren behaart. Blittfläche $2-5 \mathrm{~mm}$ breit, beiderseits stark rauh. kahl. Blatthäntchen sehr kurz. Ähre 3-6(-8) cm lang, fein, schmal. Ährchen kurz; Mittelährchen lis is mm lang. mit rom firunde grannenartigen, bis $6 \mathrm{~cm}$ langen, 
feinen Hïllspelzen und kurzer, lanzettlicher, bis fast 7 cm lang begramnter Deckspelze; Seitonän'len verkïmmert, mit grammenatigen, bis $6 \mathrm{~cm}$ lamgen Hüllspelzen und gramnenartiger, bis $2,5 \mathrm{~cm}$ langer Deckspelzr. Blïte Juni bis September.

Auf Schutt selten.

Hamburg: Wollkämmerei am Reiherstieg (Zimpel 1895!), Georgswärder (Zimpel 1896!), am Magdeburger Hafen (c Timm 1888!), Hamm (Kausch 1887), Dampfmühle Wandsbek (Röper 1908!), Uhlenhorst (Kauseh 1887!), Winterhude mehrfach (Erichsen 1885!, (\% Timm Deutsch. Bot. Ges. V. CIV [1887]), Eppendorf (Laban 1890!), bei Altona am Diebsteich (J. Schmidt 1890!), Langenfelde (Laban 1892!), Bahrenfeld 1905!!, Steilshop 1912!!, Fuhlsbiittel 1913!!, Blankenese (J. Schmidt 1910!). Kiel: am Kronshagener IVeg (A. Christiansen 1908!)。

187. (adv. 98). Hordeum compressum Grisebach Plant. Lorentz. 201 (1874).

4. Stengel kurz aufsteigend, 0,2-0,4 m hoch, ziemlich starr, glatt. Blattscheiden eng, verlängert, kahl oder an Blattsprossen die mteren fein kurzharig. Blattfläche $2-3 \mathrm{~mm}$ breit, oft eingerollt, etwas starr, unterseits glatt, kahl, oberseits kurzhaarig. Ahre aufrecht, schmal, dicht, 2-5 cm lang, mit zerbrechlicher Achse. Ährchen mit Grannen nur $1-1,3 \mathrm{~cm}$ lang; Mittelährchen nit lanzettlicher, $6-7 \mathrm{~mm}$ langer, $3-6 \mathrm{~mm}$ lang begrannter Deckspelze und grannenartigen, 7-11 mm langen Hüllspelzen; Seitenährchen mit 2-4 mm langer, gramenloser Deckspelze und bis $7 \mathrm{~mm}$ langen Hüllspelzen. Blüte Juli bis August.

Auf Schutt bei Hamburg selten.

Ha mburg: bei der Wollkämmerei am Reiherstieg (.J. N'chmidt 1894!. I)eutsch. Bot. Monatsschr. XIV. 54 [1896]; Zimpel 1895-1898 jährlich!).

188. (sp. 76). Hordeum europaeum Allioni Fl. Pedemont. II. 260 (1785).

4. Grundachse kriechend. Stengel aufrecht, seltener aufsteigend, 0,6-1,4 m hoch, unter der Thre etwas rauh. sonst glatt. Blattscheiden eng, die unteren dicht beharrt, die oberen kahl. Blattflache $t-10 \mathrm{~mm}$ breit, am Rande stark, sonst schwach rauh, oberseits behaart. Blatthäutchen sehr kurz. Ähre aufrecht, schmal, 4-10 cm lang;, mit zäher Arhse. Ïhrehen ziemlich sleich, alle fruchtbar, aiter mit dem Ansaty einer zweiten Blüte. Hïllspelzen gramnenatio mit stärkerem muteren T'eil. mit (irammo ofwal $18 \mathrm{~mm}$ lang. Deckspelzon lanzettlich. (8-) $10 \mathrm{~mm}$

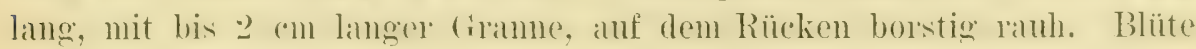
Juni bis August.

In schattigen Laubwäldern (gern mit Bromes ramosus und Triticum

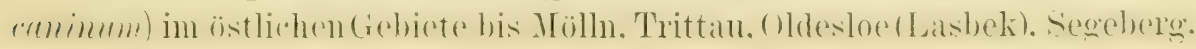


Bordesholm, Schleswig, Flensburg, Rothenkiug und Woyens nicht selten bis zerstrent, sonst nur Stormarn: Reinbek (Nolte 1822; neuerdings nicht) und im Westen im Kreise Hadersleben: Gramm (Vilandt nach Lange Bot. Tidsskr. III. 71 [1869])!!

Die erste Erwähnung der Art für das Gebiet hat Weber (Prim. Fl. Hols. 11 [1780]). Wemn das Gras von Ritter 1816 p. 50: "auf Triften und Weiden" genannt wird, so liegt eine Verwechslung vor. Bei Hamburg: Nienstedten (Sickmann Enumeratio 13) wuchs nach Sonder $H$. secalimum; trotz der Berichtigung hat Laban (Fl. v. Holst. 221 [1866]) den Standort für $H$. europaeum wieder aufgenommen. Bei Hamburg ist das Gras als Ruderalpflanze nicht gefunden worden, denn C. Timm, den A. Junge (Ver. Naturw. Unterh. VII. 99 [1890]) als Gewährsmann nennt, hat $H$. europaeum nicht gesammelt. Einige Exemplare in einem Hamburger Herbarium, die den Namen dieser Art trugen, gehörten zu H. murinum!.

189. (adv. 99). Hordeum caput Medusae Cosson in Coss. et Dur. Expl. scienc. Alg. II. 198 (1856) subsp. asperum Degen in A. u. Gr. Syn. Mitt.-Europ. Fl. II. 1. 744 (1902).

$\odot$. Stengel zu mehreren bis vielen, aufrecht oder aus knickigem Grunde anfsteigend, 0,2-0,5 m hoch, glatt. Blattscheiden kahl, glatt. Blattfläche $2-4 \mathrm{~mm}$ breit, öter eingerollt, schwach rauh. Blatthäutchen sehr kurz. Ähre $3-4 \mathrm{~cm}$ lang, aufrecht, mit nach außen gebogenen Gramnen. Ährchen zu zweien, selten einzeln, mit ciner ausgebildeten Blïte und starren Spelzen. Hüllspelzen fast lineal, allmählich in die Grame ausgezogen, mit ihr bis fast $3 \mathrm{~cm}$ lang. Deckspelze bis $7,5 \mathrm{~mm}$ lang, mit $6-8(-13) \mathrm{cm}$ langer Granne. Blüte Juni bis September.

Auf Schutt selten.

Hamburg: Wollkämmerei am Reiherstieg (Zimpel 1896!), Dampfmühle Wandsbek (Jaal) 1893!), Winterhuder Bruch (J. Schmidt 1890!), Winterhude (Laban 1884!), am Diebsteich in Altona (C. Timm, Bruns 1886!).

190. (sp. 77). Hordeum arenarium Ascherson Fl. Brandenb. I. 874 (1864). 4. Grundachse sehr weitkriechend, ausläufertreibend. Stengel aufrecht, 0,6-1,2 $\mathrm{m}$ hoch, derb, glatt, kahl, unterwärts von dichtstehenden Blattscheiden umhïllt. Blattscheiden eng, glatt, kahl. Blattfläche 5-12 mm breit, flach, seltener eingerollt, glatt, kahl. Blatthäutchen fast fehlend. Ähre 5-30 cm lang, dicht, schmal. Ährchen zu zweien oder dreien, meistens drei- bis vierblütig, 1,5-2,5 cm lang. Hüllspelzen lanzettlich, spitz, an Rücken behaart, bis $2,5 \mathrm{~cm}$ lang. Deckspelzen bis $2 \mathrm{~cm}$ lang, lanzettlich, spitz, grammenlos, dicht behaart. Blüte Juni bis August.

Auf Sandfeldern und Dünen an der Ost- und Nordsee häufig; anch auf Föhr, Amrum, Sylt, Röm, Jordsand (Nolte 1825!), Pellworm (am 


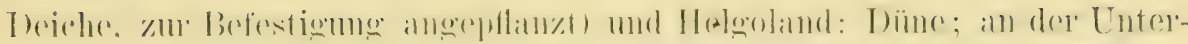

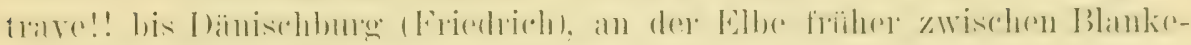
nese (Deecke, Hb. Lübeck!) mul Wedel (Sickmann, Fummeratio p. 13) bei

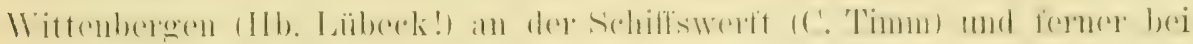

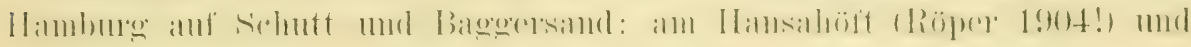
anf (ler Uhlenhorst (A. Junge 1874!, 1875!).

Die Art erseheint bei Weber als Elymus arenarius (Prim. Fl. Hols. 11 [1780]), Für Hamburg findet sich eine fragliche Notiz bei MöGler (Handb. Gewichsk. 1599 [1815]). Angeblich sammelte A. Junge sie auf Kuhwärder; seinem Herbar fehlen Belege für diesen Ort. Aus dem Bimenlande ist $H$. arenarium weiter als erwähnt nicht bekannt geworlen, da Prahl seine Angabe für Tondern: Süderlügum (Bot. Ver. Brandenb. XIV. 148) später fortgelassen lat (Krit. Fl. p. 269).

Vom Typus weicht ab:

\section{Formen.}

f. multiflorum Hallier Bot. Zeit. XXI. Beil. zu 19/20. 8 (1863). - Ahrchen mit acht bis zehn Blüten. - Helgoland: auf der Düne (Hallier).

Sonst aufgestelite Formen haben anscheinend geringen Wert. Das gilt z. B. für f. longivalvis F. v. Müller (Breviar, plant. 478 [1853]), von der ein 1845 gesammeltes Exemplar im Kieler Herbar liegt!; der ganze Bau der Pflanze entspricht dem Typus. Der Beleg stammt von Husum; Müller namnte in seiner Arbeit als Standorte Schobuill und Westeiderstedt. Ebenfalls wenig wichtig erscheint $f$. elongatum Hansen in $\mathrm{Hb}$. Hinrichsen! mit verlängerter $(-30 \mathrm{~cm})$ Ähre. Besser charakterisiert ist möglicherweise f. minus Lange Haandb. Dansk. Fl. 2. Udg. 54 (1856) mit niedrigem, am Grunde knieförmig aufsteigendem Stengel, sehr schmalen Blättern und kurzer Ähre (Rostrup, Hb. Kopenhagen!), so z. B. schon von Hansen 1846 bei Hoyer gesammelt!.

$\because$ (73) $\times 77)$. Triticum junceum $\cdots$ Hordeum arenarium $=$ Tritordeum strictum A. u. Gr. Syn. Mitt.-Europ. Fl. II. 1. 748 (1902) namnte Laban (Fl, v. Holst. 220 [1866]) als am Strande der Ostsee häufg. Eine Belegpflanze seines Herbars vom Priwall ist Triticum pungens Pers.!. Da die Hybride in Mlecklenburg und Dänemark vorkommt, wiire iln Auftreten an unserer Ostseeküste, besonders auf Fehmarn, Alsen und im Lande Oldenburg, denkbax.

54. Gattung:

Lolium.

L. Gen. plant. ed. 5. 36 (1754).

1. Pflanze 2f und rasig;, selten $\odot$; Hüllspelze selten bis doppelt so lang wie die unterste Deckspelze, in der Regel viel kürzer als diese (nur 1-1,5 mal so lang) 2

- Ptlanze $\odot$; Hüllspelze wenigstens doppelt so lang wie die unterste Deckspelze 4

2. Stengel glatt; Deckspelzen gramenlos; Pflanze $4 \ldots . . . \ldots \ldots .$. . perenne.

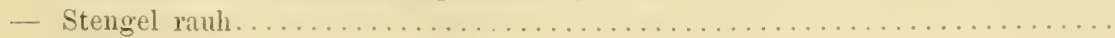


3. Deckspelzen unbegrannt; Pflanze $\odot \ldots \ldots \ldots \ldots \ldots \ldots \ldots \ldots$. . rigidum.

- Deckspelzen fast stets sämtlich oder z. T. begrannt; Pflanze 4.

4. Hüllspelzen rauh; Deckspelzen 5-7 mm lang, undeutlich nervig.

$$
\text { L. multiflorum. }
$$

L. temulentum.

- Hüllspelzen glatt; Deckspelzen etwa $4 \mathrm{~mm}$ lang, deutlich nervig... L. remotum.

191. (qsp). 15). Lolium temulentum L. Spec. plant. ed. 1. 83 (1753).

$\odot$. Pflanze ein- oder mehrstengelig. Stengel meistens aufrecht, ranh oder unterwärts glatt, $0,3-0,8 \mathrm{~m}$ hoch, kahl. Blattscheiden mehr oder weniger rauh, eng. Blattfläche $3-8 \mathrm{~mm}$ breit, unterseits schwach, oberseits stark rauh, kahl. Blatthäntchen kurz, fast fehlend. Ähre 10-18(-25) cm lang, mit wenig genäherten Ährchen: Ährchen 9-25 mm lang, vier- bis achtblütig. Hüllspelze lanzettlich, $1-1,5(-3) \mathrm{cm}$ lang, spitzlich. derb, rauh, so lang oder länger als das Ahrchen. Deckspelzen bis $7(-8) \mathrm{mm}$ lang, breit-länglich, gerundet-stumpf, grammenlos oder mit kurzer oder verlängerter Gramne, undeutlich nervig. Vorspelze breiter als die Deckspelze. Blüte Juni bis August, ofter einzeln bis Oktober.

Auf Äckern, an Wegrändern, auf Schutt und Gartenland meistens nicht selten, doch auf den Nordfriesischen Inseln nu auf Föhr (Fröhlich, Hb. Kopenhagen!; Peters, Schiötz, Arfsten).

Genannt von Weber (Prim. Fl. Hols. 11 [1780]).

\section{Formen.}

1. Deckspelze begrannt; Granne kräftig, länger als ihre Spelze:

f. macrochaeton A. Braun Flora XVII. 252 (1834). - So verbreiteter.

Zerfällt in zwei Unterformen:

f. commune nov. f. - Stengel rauh. - Hänfigste Abart. -

Dazu zählt als armblütige Form: $f$. oliganthum Godr. et Gren. Fl. France III. 615 (1856) mit zwei- bis vierblütigen Ährchen. - Stormarn: Boberg (Sonder Fl. Hamb. 78).

f. laeve nov. f. - Stengel glatt. - Hamburg (Hb. Laban!): Georgswärder (Zimpel 1893!), Königsland bei Wandsbek (J. Schmidt 1889!), Langenfelde (Laban 1892!). - Von Borchmann erwähnt (Fl. v. Holst. 66 [1856]).

- Deckspelze grannenlos oder kurz begrannt:

f. arvense Babingt. Man. Brit. Bot. 377 (1843). - So seltener.

Zerfällt entsprechend voriger Form:

f. robustum Koch Syn. Fl. Germ. et Helv. ed. 2.957 (1844). -

Stengel rauh. - So verbreiteter, doch oft spärlich. 
f. speciosum Koch a. a. 0. 957 (1844). - Stengel glatt. Hamburg: Dampfmühle Wandsbek (Zimpel 1894!, Jaap) 1895!), Steilshop 1912!!, Altona (Zimpel 1891!), Langenfelde(Laban 1892!). Kiel: Neumühlen(A.Christiansen 1908!).

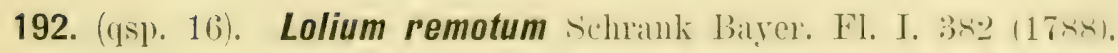

$\odot$. Stengel einzeln oder zu mehreren, anfrecht oder oft anfeteigend.

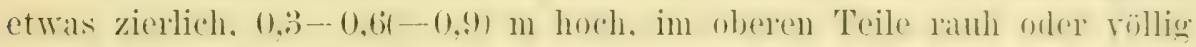
glatt. Blattscheiden eng, glatt, seltener schwach rauh. Blattlliache $2-4 \mathrm{~mm}$ breit, unterseits glatt, oberseits schwach rauh. Blatthäutchen kul\%, hreit. Ähre 6-10(-15) cm lang, locker. Ährchen 7-10 mm lang, drei- bis achlt- (bis zehn-bblütig. Hüllspelzen $7-9 \mathrm{~mm}$ lang, meistens wenig kiüzer als das Ährchen, glatt. Deckspelzen etwa $41-5$ lmm lang, hreit-länglich. stumpt. dentlicher nervig, glamnenlos oder kur\% begramnt. Blüte .Tuni bis August, einzeln später.

Anf Feldem unter Flachs früher zerstreut, doch mbeständig. neuerdings mit Abnahme des Flachsbanes viel seltener: bei Hamburg und Kiel auf Schuttland.

Die erste Feststellung für das Gebiet gab) Hornemamn (Dansk. Oece Plantel. 3. Udg. 1 [1821]).

\section{Formen.}

Die Art gliedert sich ähnlich der vorigen:

1. Deckspelzen grannenlos:

f. typicum Beck Fl. Nied.-Oesterr. I. 113 (1890). - Terbreitetste Form mit fünf- bis achtblütigen Ährchen.

Bei dieser wie bei der folgenden Form können als Unterformen auftreten:

f. complanatum Koch Syn. Fl. Germ. et Helv. ed. 2. $95 \tilde{i}$ (1844). - Ährchen sieben- bis neumblütig, mehr abstehend als beim Typus. - Hamburg (Sonder, Fl. Hamb. 77 [1851]): Steilshop!!.

f. oliganthum Beck a. a. O. I. 113 (1890), - Ährchen dreibis fünfblütig. - So bisher im Gebiete nicht festgestellt.

- Deckspelzen kurz begrannt:

f. aristatum Döll Fl. v. Baden 113 (185̃7). — Selten.

Zerfällt in zwei Formen:

f. asperum Ascherson Fl. Brandenb. I. 876 (1864). - Stengel rauh. - Hamburg: Georgswärder (Zimpel 1893!), Winterhuder Bruch (Laban 1892!, Hb. W. Hansen). Neustadt (Schröder 1831, Hb. Kiel!). Flensburg: Harrislee (Prahl 1875!). 
f. laeve Ascherson a. a. O. I. 876 (1864). - Stengel glatt. - Hamburg: Georgswärder (Laban!). Kiel (Nolte 1832! unter L. temulentum).

193. (.1). 78). Lolium perenne I. . viec. plant. ed. 1. 83 (1753).

2. Frundachse dichtrasig. Sitengel anfrecht oder foft aus liegendem (trunde) aufsteigend, 0.2-0.6 $\mathrm{m}$ loch, glatt, kahl. Blattscheiden eng, glatt, kahl. Blattfläche $(1-) 2-5 \mathrm{~mm}$ breit, selten gefaltet, glatt oder oberseits schwarh rauh. Blatthäutchen his $1 \mathrm{~mm}$ lang, breit abgeschnitten. Ïhre 4-15(-30) cun lang, aufrecht odfr seltener bis überhängend, wenig dicht bis dicht. Ährchen bis $2 \mathrm{~cm}$ lang, (3-)6-10(-16)blütig. Hüllspelze $7-8(-10) \mathrm{mm}$ lang, spitzlich, meistens viel kürzer als das Ährchen. glatt. Deckspelzen (t- í)- (; mm lang, spitz lis stmmpflich, undeutlich nervig, gramnenlos. Blïte Juni bis November.

Auf Wiesen und Weiden, an Wegrändern und Abhängen häufig, auch auf den Nordfriesischen Inseln (selbst auf Helgoland).

VTeber nennt als erster Florist unseres Gebiets L. pereme (Prim. Fl. Hols. 11 [1780]).

\section{Formen.}

1. Ährchen sieben- bis zehublütig:

f. typicum Beck Fl. Nied.-Oesterr. I. 112 (1890). — Häufigste Form.

Dazu gehört in der Regel als Unterform:

f. cristatum Döll Fl. v. Baden 116 (1857). - Ährchen dicht gedrängt, weit von der Achse absteliend. Zerstrent, stellenweise nicht selten, selbst auf Helgoland (Brody!) und Sylt: Westerland!!. - Hierher zählt vielleicht $f$. monstrosum Hallier.

f. Tongiglume Grantzow Fl. Uckerm. 351 (1880). - Hüllspelze so lang wie das Ährchen. - Hamburg: Steilshop!!

- Ährchen mehr- oder wenigerblütig .................. 2

2. Ährchen drei- bis vierblititig:

f. pauciflorum A. u. Gr. Syn. Mitt.-Europ. Fl. II. 1. 754 (1902). - An trockenen Orten zerstreut; doch selbst auf Helgoland gesammelt.

-- Ährchen 12-16blïtig; Pflanze sehr kräftig:

f. orgyiale Döll a. a. O. 116 (1857). -- Zerstreut, stellenweise nicht selten. - Hierher oder zu $f$. polyanthum Beck a. a. 0 . I. 112 (1890) gehört $f$. multiflonum Sonder Fl. Hamb. 76 (1851). 


\section{Mißbildungen.}

f. m. compositum Sonder Fl. Hamb. 76 (1851). - Thre aus den Achseln der Hüllspelzen rerästelt. - So nicht selten, schon genamnt von Mößler (Handb. Gewächsk. 116 [1815]) als f. ramosum; hierher anch f. remosum Nolte bei Ohl (Pflanzen Kiels 22 [1889]).

f'. m. riviparm Koch Syn. Fl. Germ. et Helv. ed. 2. 956 (1844). Ährchen in Laubsprosse answachsend. - Hamburg: Holstenbrauerei in Altona (Brick 1892!), Billwärder a. d. Bille (Röper 1912!). Lübeck: Niendorf a. O. (Hirth 1903!)。 Husum: Aue und Flensburger Chaussee (F. v. Mü̈ller).

f. m. palaeacerm Döll a. a. O. 116 (1857). - Ährchen einseitig verschoben, kammförmig gestellt. - Kiel: Gaarden (A. Christiansen 1912!) (bei ff. cristatum).

f. m. ramiflorum nov. f. - Pflanze mit oberwärts ans den Knoten ästigem Stengel. - Hamburg: Winterhude (Beyle 1884!).

194. (adv. 100\%. Lolium rigidum (iandin Agrost. Helv. I. 334 (1811).

$\odot$. Stengel zu mehreren, am Grunde stark ästig, 0,3-0,5 m hoch, aufsteigend, unter den oberen Knoten weit herab rauh. Blattscheiden schwach rauh. Blattfläche 2-3 mm breit, flach, zuweilen eingerollt, glatt oder schwach rauh. Blatthäutchen bis $1 \mathrm{~mm}$ lang, stumpf. Ähre bis $15 \mathrm{~cm}$ lang, mit vorwärts rauher Achse. Ährchen bis $13 \mathrm{~mm}$ lang, vier- bis sechsblütig. Hüllspelzen etwa $8 \mathrm{~mm}$ lang, bis fast so lang wie das .̈hrehen oder kïrzer. spitz. starr. Deckipelzen bis 8 mm lang. stmmptlich. gegen das obere Ende breit weißhäutig, grannenlos. Blüte Juli.

Mit Kleesaat selten eingeführt.

Lauenburg: am Rande eines Kleeackers bei Hakendorf am Schaalsee 1912!! (det. Hackel).

Die Art dürfte mit Saat aus Italien oder Südfrankreich eingeführt sein. Eine frühere Angabe aus dex Hamburger Adventivflora (Deutsch. Bot. Monatsschr. XIV. 54 [1896]) bezieht sich nach den vorliegenden Exemplaren (Georgswärder 1893!, Ausschlägerweg 1893!, Dampfmühle Wandsbek 1895!) auf $L$. temulentum f. arvense sbf. robustum.

\section{Mißbildungen.}

f.m. compositum nov. f. - Ähre am Grunde mit Seitenähren. - Lauenburg: Hakendorf!!.

Lolium strictum Presl. Gram. et Cyperac. Sic. 49 (1820) gehört möglicherweise der Hamburger Adrentivflora an. Doch sind die in Betracht kommenden Pflanzen, von Zimpel 1896 auf dem Kl. Grasbrook! und bei der Dampfmühle Wandsbek! gesammelt, nicht mit völliger Sicherheit hierher zu ziehen. 
195. (sp. 79). Lolium multiflorum Lamark Fl. Franç. III. 621 (1778).

4. Grundachse dichtrasig. Stengel aufrecht oder anfsteigend. $0.2-0.8-11 \mathrm{~m}$ hoch, unterwärts glatt, oberwärts glatt oder in der Regel rauh. kahl. Blattscheiden eng, glatt oder schwach rauh. Blattfläche $3-6(-8) \mathrm{mm}$ breit, unterseits glatt. olerseits schwach rauh. Blatthäutchen fast fehlend. Ähre $10-40 \mathrm{~cm}$ lang. ziemlich locker, scltener dicht. Ährchen $1-1.5(-2.3)$ cm lang. mit (3)-j6-12) -20$)$ Blüten. Hüllspelzen $5-9$ (-13) mm lang. spitz lis stmmpflich. etra ron halber Ährchenlänge. Decksuelzen $(2,5-5-5$ wenigstens z. T. begramut. spitzlich. Blüte Juni bis August, später spärlich.

Auf Wiesen und Weiden, an Wegrändern. auf Schuttplätzen nicht selten bis häutig und eingebürgert. doch auf den Nordfriesischen Inseln nur auf Helgoland (Brody)!!. Föhr: Goting (hertelheim), Anrum: Wittdün (Kuckuck und Polgar 19on!) und Sylt: Westerland, Feitum und Gr. Morsum!.. Zuerst erwähnt ron Sonder (Fl. Hamb. 77 [1851]).

\section{Formen.}

Von Formen wurden beobachtet:

f. Tongiaristatum A. 11. Gr. Syn. Mitt.-Europ. Fl. ПI. 1. 758 (1902). Obere Deckiselzen mit ihnen an Lünge gleichkommender oder sie übertreffender Granne. - Verbreitetste Form.

Dazu als Unterformen:

f. cristatum C. Timm Deutsch. Bot. Ges. IV. CLXIX. (1887). Ährchen dicht gedrängt, in kurzer Ähre. - Hamburg: Winterhuder Alsterufer (C. Timm). Kiel: Rotenhahn und Rönne (A. Christiansen!).

f. microstachyum Uechtr. 57. Ber. Schles. Ges. 334 (1880). Ährchen klein, drei- bis fünfblütig. - Kiel: Gaarden (A. Christiansen!).

f. submuticum Mutel Fl. Franç. IV. 139 (1837). - Nur einzelne Deckspelzen kuxz begrannt. - Ziemlich selten. Hamburg: Wollkämmerei am Reiherstieg (Zimpel 1896!), Langenfelde (Brick 1893 !). Fuhlsbüttel 1912!!. La u en burg: Börnsen!!. Plön: Timmdorf 1912 !!. Kiel: Rotenhahn, Bruchs (A. Christiansen!).

f. muticum DC. Fl. Franc. 3. ed. T. 286 (1816). — Alle Deckspelzen unbegrannt. - Stormarn: Ladenbek!!. Hamburg: Winterhude (C. Timm 1870, Laban 1873!.. Hammerbrook (Jaap!). Fuhlsbüttel!!. Kiel: Gaarden (A. Christiansen!).

\section{Mißbildungen.}

f. m. ramosum Guss. Fl. Sic. Syn. I. 59 (1842). - Ähre (besonders am Grunde) rispig rerzweigt. - Hamburg: Wollkämmerei Reiherstieg 
(Zimpel 1893!), Georgswitrder (Zimpel 1894!), Hammerbrook (Jaap) 1891!). Hamm (Kausch 1888!), Uhlenhorst (c. 'Timm vor 1880), Winterhude (\%impel 1893!), Eppendorf (Kanseh 18s8!). Sitormarn: Poplenbuittel (A. Moln!). Lübeck: Niendorf a.d. Stecknitz 1905!!. Kiel (A. Christiansen 1907!). Hadersleben: Christiantal (Prahl 1872!).

\section{$\times(\mathrm{p}) .57 \times 78)$. Festuca pratensis $\times$ Lolium perenne $=$ Festulolium}

ascendens A. u. Gr. Syn. Mitt.-Europ. Fl. II. 1. 768 (1902).

4. Grundachse ziemlich dicht-bis lockerrasig. Stengel zu mehreren. aufrecht oder meistens autsteigend. 0,2-0,7 m hoch, glatt, kahl. [3]attscheiden glatt, kahl. Blattfläche 3-6 mm breit, schwach rauh. Blatthäutchen kurz, breit. Ährehenstand fast ährig oder offer traubig, seltener rispig mit verlängerten, unteren Ästen. Ährchen etwas entfernt, 1-2 (-2,6) (:m lang, 7-9-13)blïtig, schief zur Achse gestellt, fast sitzend oder (in der 'T'raube) 1 - 5 mm lang gestielt. Hüllspelzen selten fast gleich, in der Regel sehr ungleich. die obere 5-8 mm lang, lanzettlich, spitzlich. ein- bis dreinervig, die untere fehlend oder $1,5-6 \mathrm{~mm}$ lang, spitzlich, drei- bis fünfuervig. Deckiselzen lanzettlich, bis 6 mm lang. Antheren etwa $2 \mathrm{~mm}$ lang. Frucht in der Regel fehlschlagend. Blüte Juni bis August, später seltener.

Auf Weiden und Grasplätzen, an Tregrändern und auf Schuttland ziemlich selten, doch wohl vielfach übersehen.

Lauenburg: bei Krümmel 1911!!. Hamburg: am Eppendorfer Moor (Sonder, Hb. Kopenhagen!), am Dammtor (Sonder 1840, Hb. Lübeck!; dazu wohl: ,am Stadtwall“ nach C.Timm), Hamm (Sonder), Horn!!, Hinschenfelde (C. Timm), Dampfmülıle Wandsbek (Zimpel 1895!). Pinneberg: Utersen (C. Christiansen 1888!). Oldesloe: Fußsteig nach dem Bremner Moore 1912!!. Lübeck: Niendorf a. 0. (Hirth 1903!). Preetz: zwischen Pohmsdorferfeld und Rönnerholz (A. Christiansen!). Kiel: Rotenhahn!. an schilksee!. Meimersdorf!, am Fuhlensee!, Gettorf! und Schwansen: Schuby (A. Christiansen!). Flensburg: Ekensund (Prahl). Hadersleben: an der Föhrde (A. Christiansen 1907!).

Zuerst sicher erwähnt durch Sonder (FI. Hamb. 65 [1851]).

Schon Nolte führt Festuca loliacea auf. Seine Belege gehören zu F. pratensis, Lolium perenne und L. multiflorum. Trotzdem zieht Kunth sie unter die Kreuzung (Fl. v. Schlesw.-Holst. 777 [1887]): Lauenburg in der Aue (Thun) (= Festuca pratensis!), Rotenhaus (Nolte) (=F. pratensis!), Friedrichsort (Nolte) (= Lolium perenne!), Tondernsche Marsch (Hemniges) (=L. perenne!) und Gravenstein (Hansen) (=L. multiflorum!). Für Hornemanns Angabe: Propstei (Oec. Plantel. 3. Udg. II. 123 [1837]) enthält das Kópenhagener Herbar keine Belege; dasselbe gilt für die Ortsnotizen der Klattschen Flora (FI. v. Lautnb. 165) [1865]) : Lauenburg (Claudius) und (ir. Saran (Luther), sowie für eine Erwähnung aus der Eutiner Gegend: Krummsee (Erichsen nach C. Timm Deutsch. Bot. Ges. VII (95) [1889]), (fehlt im Herbar Erichsen). 
Tom Typus weicht ab:

Formen.

f. paniculatum Sonder Fl. Hamb. 65 (1851). - Ährchen in einer Rispe mit ährenähnlichen Ästen. - Lauenburg: Krümmel!! Hamburg (Sonder; ohne Standort). Kiel: am Schilksee (A. Christiansen 1912!).

\section{ว๊ร. Gattung:}

\section{Lepturus.}

R. Brown Prodr. Fl. Jov. Holland. I. 207 (1810).

196. (s). 20$). \quad$ Lepturus incurvatus Trin. Fuml. Agrost. 123 (152()).

$\odot$. Stengel einzeln oder zu wenigen, seltener sehr zahlreich, in der lirgel statk verzweigt, antrecht onler bogig antsteigend. zuweilen am

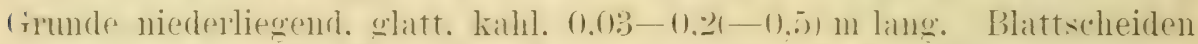

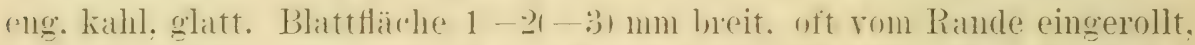
menterseits glatt. oberseits und am liande ratuh. Bhattloäutchen bis $1 \mathrm{~mm}$ lang, breit abgeschnitten. Ähre $(2-) 4-8(-10) \mathrm{cm}$ lang, sehr schmal. Ährchen 5-7 mm lang, der Achse angedrückt, mit einer Blüte. Hüll-

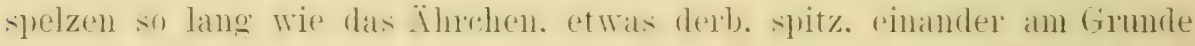
sehr genähert, unterwärts dentlich nervig. Deckspelze $\check{-6} \mathrm{~mm}$ lang, schmallanzettlich, häutig. Antheren ㄹ mm lang. goll,weib. Blüte Juni und Juli, selten bis Oktober.

Auf etwas fenchtem. sandigem oder tonig-sindigrem Borden am strande der Nord- und Ostsee zerstrent, stellenweise in enormer Menge.

0stsee. Lübeck: Priwall bei Travemünde (Häcker 1844!, Nielsen

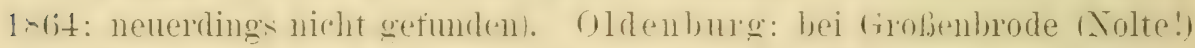
vielfach!!. bei Heiligenhaten (Prehn) vieltach!!. Fehnarn: am Burger

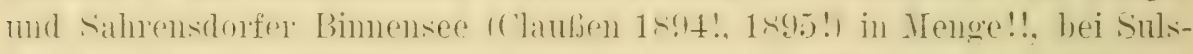
dorf (J. Schmidt 1892!), am Salzensee und am Fastensee bei Westermakelsdorf in gecdulosenem Beständen 1911!!. Plön: am sehlendorter

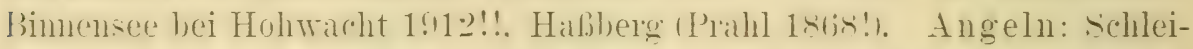

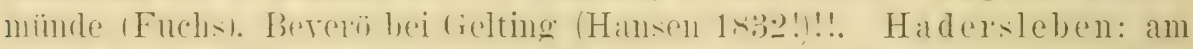
Halker Noor (A. Christiansen 1910!)!!, Aarö (J. Schmidt!).

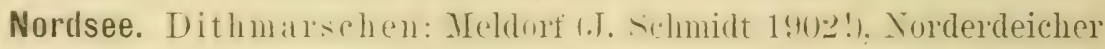
Aubendeich hei Tresenlluren (Tribeken!). zwishen Herwigenkoog und

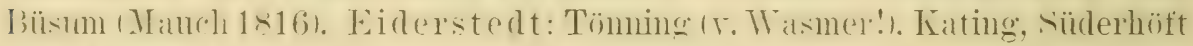
mol ording 1905!!. If alligen: Beenshallig (Nolte 18.5!). Hallig Koller (Teers 1833!). Föhr (Buchenaul. Amrum (Nolte 1825!. Buchenau). sylt (Spicker nach Lange, Buchenau). Röm (Borst, Prahl 1874!) 1904!!. 
Mauch entdeckte das Gras im Gebiet 1816; von Nolte stammt die

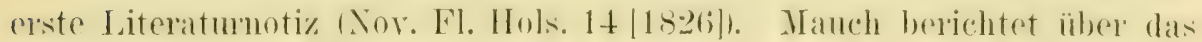
Vorkommen bei Bü̈smm $18+1$ (Neues staatsbürorel. Magazin X. 520).

Wenn Knuth (Fl. Nordfries. Ins. 151 [1895]) das Gras als auf den Inseln verbreitet nennt, so ist das ein Ausdruck, der den Häufigkeitsgrad nicht trifft; die Art ist ziemlich selten, am verbreitetsten auf Röm, wo sie nicht nur ummittelbar am Strande, sondern auch zwischen alten und jungen Dünen sich findet ("Wiesenzone" bei Engell in Bremer Abhandl. XVII. 2. 244 [1903]). Für "Gr. Brode" (Nolte, Handschriftl. Nachträge zu den Novitien) vermutet Reichenbach (Vorles.-Verz. Hamb. 20 [1881]), daß Brodau bei Neustadt als Fundort gemeint sei; es handelt sich aber um Großenbrode bei Heiligenhafen.

\section{Formen.}

Bei uns nur die Rasse:

var. vulgatus A. u. Gr. Syn. Mitt.-Europ. Fl. II. 1. 764 (1902).

Zerfällt in zwei Formen:

f. subcurvatus A. i. Gr. a. a. O. II. 1. 764 (1902). - Pflanze niedriger, mit bogig aufsteigenden Stengeln und säbelförmig gebogenen Ähren. - So an trockeneren Orten mit spärlichem Pflanzenwuchs.

Dazu als Unterform:

f. multicaulis nov. f. - Pflanze mit sehr zahlreichen (bis über 100) allseitig auseinandergestellten Stengeln, fast rasenbildend. - Oldenburg: am Steinwarder bei Heiligenhafen!!, am Fehmarnsund nördlich von Großenbrode!!. Fehmarn: am Fastensee!!.

f. strictus Buchenau Abhandl. Naturw. Ver. Bremen XV. 293 (1901). - Pflanze in der Regel höher, mit geraden, wenigstens im oberen Teile aufrecht stehenden Stengeln und gerader Ähre. - So mehr an feuchteren Orten und in dichtem Pflanzenwuchse.

Dazu als Unterform:

f. gracilis A. u. Gr. a. a. O. II. 1. 764 (1902). Pflanze mit einfachem oder sehr schwach verzweigtem, fadendïmnem, sehr zierlichem Stengel und kleinen Ährchen. - So mit der Form selten. Oldenburg: bei Heiligenhafen (J. Schmidt 1892!)!!, Großenbrode!!. Hadersleben: am Aarökalv (A. Christiansen 1909!). Röm: am Porrenpriel und bei Jurre (J. Schmidt!).

In der Regel treten beide Formen, $f$. subcurvatus und $f$. strictus, an ihren Standorten gemeinsam auf; nur ausnahmsweise fehlt eine ron ihnen, wo die andere rorkommt. Oft ist die eine Form weit häufiger als die andere, so anscheinend (früher) $f$. subcurvatus auf dem Priwall. Wenn Reichenbach (Agrost. 24 [1850]) „L. filiformis Trin." aus 
Holstein aufführt, so ist die Angabe begründet. Wemn er aber am gleichen Orte ..L. incurvatus Trin." aufführt mit der Standortsangabe ..Beneschallig" (gemeint ist die rerschwundene Beenshallig), so liegt eine Terwechselung dieser südlichen Rasse vor, wahrscheinlich mit der ihr habituell ähnlichen $f$. subcurvatus.

\section{Gattung.}

\section{Psilurus.}

Trinius Fund. Agrost. I. 73 [1820]).

197. (adv. 101). Psilurus aristatus Dur.-Jouve Soc. Bot. France XIII. 132 (1866).

$\odot$. Stengel zu mehreren, am Grunde rerzweigt, anfrecht oder aufsteigend, zart, 0,1-0,4 m hoch, glatt, kihl. Blattscheiden eng, glatt, kahl. Blattfläche 1(-2) mm breit, kahl. glatt. Blatthäutchen sehr kurz, zuweilen bis $1 \mathrm{~mm}$ verlängert. Ähre sehr fein, 3-10(-20) $\mathrm{cm}$ lang, mit gebogener, in der Regel glatter Achse. Ährchen schmallanzettlich, der Achse angedruickt, ohne Granne etwa $5(-6) \mathrm{mm}$ lang, einblïtig, mit Ansatz einer zweiten Blüte. Hüllspelze schuppenartig, nicht $1 \mathrm{~mm}$ lang. Deckspelze $5 \mathrm{~mm}$ lang, am Rücken rauh. spitz ansgezogen in eine $2--6 \mathrm{~mm}$ lange, gerade, feine Granne. Antheren einzeln. Blüte August bis Oktober.

Auf Schutt bei Hamburg selten.

Hamburg: bei der Wollkämmerei am Reiherstieg (Zimpel 1895!) (Deutseh. Bot. Monatsschr. XVI. 54 [1898]). 


\section{Gattungsregister.}

\begin{tabular}{|c|c|}
\hline & 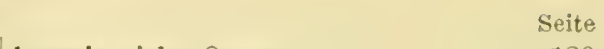 \\
\hline egilops siehe Triticum ......... 290 & Leersia siehe Oryza. \\
\hline $\operatorname{era} \ldots \ldots \ldots \ldots \ldots \ldots \ldots \ldots \ldots \ldots \ldots \ldots \ldots$ & Lepturus......... \\
\hline gropyrum siehe Triticum ......... 290 & Lolium ..... . \\
\hline grostis . . . . . . . . . . . 1677 & \\
\hline ra siehe Aera ............... 201 & \\
\hline lopecurus . . . . . . . . . . . . 154 & Milium.................... \\
\hline nmophila siehe Calamagrostis...... 177 & Molinia . . . . . . . . . . . . . . . 21: \\
\hline ndropogon .................. 132 & Miihlenbergia siehe Agrostis . . . . . 16 \\
\hline nthoxanthum . . . . . . . . . . 126 & Nardus . .................. 15 \\
\hline pera siehe Agrostis .......... 167 & Nephelochloa siehe Poa........... 22 \\
\hline rrhenatherum siehe Avena ....... 189 & Oryza .................... 12 \\
\hline rủndo .................. 212 & 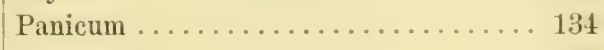 \\
\hline vena . . . . . . . . . . . . . . 189 & Paspalus ................... \\
\hline nannia ................ 147 & . \\
\hline rachypodium .. . . . . . . . . 287 & Phalaris ......... \\
\hline Briza.................... 240 & $u m \ldots \ldots \ldots \ldots \ldots \ldots \ldots$ \\
\hline 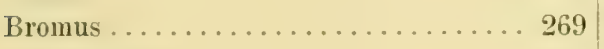 & nites siehe Arundo ........ \\
\hline rostis ............ 177 & \\
\hline$a \ldots \ldots \ldots \ldots \ldots \ldots \ldots \ldots \ldots \ldots \ldots$ & Polypogon .................. \\
\hline$\ldots \ldots \ldots \ldots \ldots \ldots \ldots \ldots \ldots \ldots \ldots \ldots$ & a siehe Calamagrostis ......... \\
\hline ostis siehe Mibora........ 153 & $\mathrm{~s} \ldots \ldots \ldots \ldots \ldots \ldots \ldots \ldots$ \\
\hline$\ldots \ldots \ldots \ldots \ldots \ldots \ldots \ldots \ldots$ & allia siehe Lepturus ......... \\
\hline phorus siehe Weingaertneria... 209 & mus $\ldots \ldots \ldots \ldots \ldots \ldots$ \\
\hline$\ldots \ldots \ldots \ldots \ldots \ldots \ldots \ldots \ldots \ldots$ & 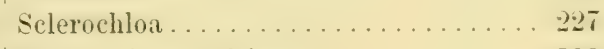 \\
\hline as................ 267 & 1e Triticum ............. \\
\hline$\ldots \ldots \ldots \ldots \ldots \ldots \ldots 224$ & siehe Bromus......... \\
\hline $\operatorname{um} \ldots \ldots \ldots \ldots \ldots \ldots \ldots \ldots$ & he Panicum............ \\
\hline$\ldots \ldots \ldots \ldots \ldots \ldots \ldots 215$ & a.................... \\
\hline ehe Eragrostis......... 221 & siehe Andropogon ........ \\
\hline ehe Oryza .......... 120 & ................. \\
\hline ( $\ldots \ldots \ldots \ldots \ldots \ldots \ldots \ldots$ & he Stupa.............. \\
\hline s siehe Hordeum .......... 306 & $\ldots \ldots \ldots \ldots \ldots \ldots \ldots \ldots$ \\
\hline$\ldots \ldots \ldots \ldots \ldots \ldots \ldots 221$ & Syntherisma siehe Panicum ........ \\
\hline$\ldots \ldots \ldots \ldots \ldots \ldots \ldots \ldots 248$ & $\operatorname{Tr}$ \\
\hline $\operatorname{lium} \ldots \ldots \ldots \ldots \ldots \ldots \ldots \ldots \ldots$ & a siehe Sieglingia .......... \\
\hline$\ldots \ldots \ldots \ldots \ldots \ldots \ldots 211$ & $m \ldots \ldots \ldots \ldots \ldots \ldots \ldots$ \\
\hline . 243 & \\
\hline 10rum ............... & $u m \ldots \ldots \ldots \ldots \ldots \ldots$ \\
\hline (ch & $\ldots \ldots \ldots \ldots \ldots \ldots \ldots \ldots$ \\
\hline 187 & he Festuca..... \\
\hline . & \\
\hline han & Zea \\
\hline & \\
\hline
\end{tabular}




\section{Literatur.}

1. Alpers, F.: Beiträge zur Flora ton Sylt. Abhandlungen Naturw. Ter. Bremen XII. 1. $137 / 140$ (1894).

2. Arcangeli, G.: Flora Italiana. Turin und Rom 1894.

3. Ascherson, P.: Flora der Prorinz Brandenburg. Berlin 1864.

4. - - Noch einige Worte über Festuea loliacea Auct. Botanische Zeitung XXII. $125 / 127$ (1864).

5. _ - _ : Referat in Justs Botanischem Jahresbericht II. 1024 (1874).

6. - - _ Sitzungsherichte in Abhandlungen Botan. Ver. Prorinz Brandenburg XXXV1. p. XXV (1891).

7. - - - Bemerhungen über einige Potentillen und andere Pflanzen West- und Ostpreubens. Abhandl. Botan. Ter. Prorinz Brandenburg XXXII. 129/172 (1890).

8. - - : Ubersicht der Pteridophyten und Siphonogamen Helgolands. Wissenschaftliche Neeresuntersuchungen. Neue Folge Band IT. Heft 1. 91/140 (1900).

9. - - Legitops speltoides Jaub. u. Spach und ihr Vorkommen in Europa. Magyar Bot. Lapok. I. 1. 6/12 (1902).

10. - - und Graebner, P.: Flora des Nordostdeutschen Flachlandes. Berlin 1898/99.

11. - - - _ - Synopsis der Mitteleuropäischen Flora. Band II. 1. Leipzig 1898/1902.

12. Baenitz, C.: Uther Oryza clandestina Al. Br. forma inclusa und forma patens Wiesb. Deutsche Botanische Monatsschrift XV. 19/21 (1897).

13. Beneken, F.: Bromus serotinus n. sp. Botanische Zeitung II. 724/725 (1845).

14. Bentham, G.: Flora Australiensis. Band VII. London 1878.

15. Borchmann. F.: Holsteinische Flora. Kiel 1856.

16. Britton, X.. und Brown, A.: Illustrated Elora of the Nortleru Lnited States. Canada and the British Possessions. Nerr-York 1896.

17. Promn. P.: Prodromus Florae Novae Hollandiate th Insulae Van-Diemen. Nüruberg 1827.

1๕. Burchenau. F.: Tergleichung der nordiriesischen Inseln mit den ostfriesischen in floristischer Beziehung. Abhandlungen Naturw. Ter. Bremen IX. Heft 4. 361/384 (1887).

19. - - Die Terbreitung von Oryza clandestina Al. Braun. Botanische Zeitung LII. 83/96 (1894).

20. - - Über zwei Gräser der ostfriesischen Inseln. Abhandlungen Naturw. Ver. Bremen XV. Heft 3. 285/296 (1901).

21. - - Flora der Ostfriesischen Inseln. 4. Aufl. Leipzig 1901.

2.2. Buek. J. X.: Tersuch eines Terzeichnisses der un Hamburg wildwachsenden Pflanzen. Hoppes Botanisches Taschenbuch 1801. 86/113.

23. Coste, H.: Flore descriptive et illustrée de la France. Band III. Paris 1906.

24. Dalla-Torre. K. W. v.: Die Flora der Insel Helgoland. Berichte des Taturwissenschaftl.mediz. Tereins in Innslıruck. XVIII. 1/30 (1889).

25.) Desfontaines, R.: Flora Atlantica. Paris 1800. 
26. Döll, J. Ch.: Rheinische Flora. Frankfurt a. M. 1843.

27. - _ : Flora des Großherzogtums Baden. Band I. Karlsruhe 1857.

28. - - : Gramineae I. In: Martius: Flora Brasiliensis II. 2. München 1877.

29. Domin, K.: Monographie der Gattung Koelevia. In: Bibliotheca Botanica. Stuttgart 1907.

30. Dosch, L., und Scriba, J.: Excursionstlora des Grobherzogtums Hessen. Giefen 1888.

:31. Dreves, F., und Hayne, F. G.: Botanisches Bilderbuch für die Jugend und Freunde der Ptlanzenkunde. Band III. Leipzig 1798.

32. Fiek, E.: Flora von Schlesien. Breslau 1881.

:33. Fischer-Benzon, R. v.: Über die Flora des südwestlichen Schleswig und der Inseln Führ, Amrum und Nordstrand. Schriften Naturw. Ver. Schleswig-Holstein II. 65/116 (1876).

$\therefore 4$.

— : Iltere Arbeiten über die Flora von Schleswig-Holstein. Schriften Naturw. Ver. Schleswig-Holstein VIII. 3/15 (1889).

3i. - - _ Altdeutsche Gartenflora. Kiel und Leipzig 1894.

i3;. - - und Steinvorth, J.: Über die Flora der Ungegend ron Hadersleben. Programm der Lateinischen Schule zu Hadersleben 1873.

37. Focke, IV. O.: Uber Lotium festucaceum Link (Festuca loliacea Huds.). Botanische Zeitung XXII. 109/112 (1864).

3: - - _ A Anterungen der Flora an der Nordseeküste. Abhandlungen Naturw. Ver. Bremen XVIII. 1. 175/181 (1905).

i!). Friedrich, P.: Flora der Ungegend von Lübeck. Programm des Katharineums in Lübeck 1895.

40. - - : Nachträge zur Flora von Lübeck. Mitteilungen der Geograph. Gesellschaft und des Naturhistorischen Huseums in Lübeck. 2. Reihe, Heft 14 (1900).

41. Fries, E.: Summa Vegetabilium Scandinaviae. Stockholm und Leipzig 1846.

4:. Gaulin, J.: Flora Helvetica. Band I. Zürich 1828.

13. Graebner, P.: Die Ptlanze. In: Landeskunde der Provinz Brandenburg. Band I. Berlin 1909.

44. Grenier und Godron: Flore de France. Band III. Paris 1855.

45. Hackel, E.: Monographia Festucarum Europaearum. Kassel 1882.

46. - - : Gramineae. In: Engler und Prantl: Natiirliche Pflanzenfamilien II. 2 (1887).

47. - - Zur Biologie von Poa annu L. Österr. Bot. Zeit. LIV. 273/278 (1904).

4h. - - Über Kleistogamie bei Gräsern. Osterr. Bot. Zeit. LVI. 81/88, 143/154, 180/186 (1906).

49. Häcker, G. R.: Lübeckische Flora. Lübeck 1844.

5). - _ - Zusätze und Verbesserungen zur Lübeckischen Flora. Archir des Vereins der Freunde der Naturgeschichte in Mecklenburg XI. 133/135 (1857).

i1. Hallier, E.: Flora von Helgoland. Hamburg 1863.

¡2. - - - Vollständige Aufzählung und kritische Besprechung der phanerogamischen Flora Helgolands. Botanische Zeitung XXI. Beilage zu Nr. 19/20. 1/18 (1863).

5.3. Hansen, G.: Das Amt Bordesholm im Herzogtume Holstein. Kiel 1842.

5. Hausknecht, C.: Über Panicum ambigum Guss. (sub Setaria). Osterr. Bot. Zeit. XXV. $345 / 348$ (1875).

5i. Hegi, G.: Illustrierte Flora von Mitteleuropa. Band I. München 1906.

ix. Hennings, P.: Standortsverzeichnis der bei Hohenwestedt vorkommenden selteneren Pflanzen. Schriften Naturw. Ver. Schleswig-Holstein. II. 1. 141/146 (1876).

¿. - - - Standortsverzeichnis der Gefäßpflanzen in der Umgebung Kiels. Schriften Naturw. Ver. Schleswig-Holstein. II. 1. 147/208 (1876).

is. - - - Nachtrag zum Standortsverzeichnis der Gefäbptlanzen in der Umgebung Kiels. Schriften Naturw. Ter. Schleswig-Holstein. IV. 1. 71/98 (1850) 
59. Höck. E.: Brandenburger Buchenbegleiter. Abhandlungen Botan. Ver. Provinz Brandenburg XXXVI. 7/50 (1894).

60. - - Ankömmlinge in der Pflanzenwelt Mitteleuropas während des letzten halben Jahrhunderts. Beihefte zum Botan. Zentralblatt XVII. Heft 1. 195/210 (1904), XXVI. 391/433 (1910).

61. Hornemann, J. W.: Dansk Oeconomisk Plantelaere. 1. Udg. (1796), 2. Udg. (1806), 3. Udg. I (1821), II (1837).

62. - - : Flora Danica. Bd. VIII. Heft 23 (1808), Heft 24 (1810); Bd. IX. Heft 25 (1813), Heft 26 (1816), Heft 27 (1818); Bd. X. Heft 28 (1819), Heft 29 (1821), Heft 30 (1823); Bd. XI. Heft 31 (1825), Heft 32 (1827), Heft 33 (1829); Bd. XII. Heft 34 (1830); Bd. XIII. Heft 37 (1836), Heft 38 (1839).

63. - - - : Bemaerkninger angaaende Forskjelligheden af Vegetationen i de danske Provinser. Vidensk. Selsk. physikal. Skrift. I. Deel, I. Haefte. 153/208 (1821).

64. Hübener, J. W. P.: Flora der Umgegend von Hamburg. Hamburg 1846.

6อ. Jaap, O.: Zur Gefäßpflanzenflora der Insel Sylt. Allg. Bot. Zeitschr. IV. 1. 2 (1898).

(66. - - Einige Notizen zur Gefäßpflanzenflora der nordfriesischen Insel Röm. Deutsche Bot. Monatsschr. XX. 2. 28/29 (1902).

67. Junge, A.: Die Ruderal- und Baggerflora hiesiger Gegend. Verhandl. des Vereins für Naturw. Unterhaltung zu Hamburg VII. 52/109 (1890).

6s. - - - Nachträge. Verhandl. des Vereins für Naturw. Unterhaltung zu Hamburg VIII. $57 / 69$ (1894).

69. Junge, P.: Die Gefäbptlanzen des Eppendorfer Moores bei Hamburg. Verhandl. Naturw. Ver. Hamburg 3. Folge XII. 30/76 (1904).

70. - - Beiträge zur Kenntnis der Gefäßpflanzen Schleswig-Holsteins. Jahrb. Hamb. Wissenschaftl. Anstalten XXII. 49/108 (1905).

71. - - Z Zur Flora des Elbgebiets zwischen Harburg und Bleckede. Verhandl. Naturw. Ver. Hamburg 3. Folge XVII. 38/51 (1909).

72. - - Flora von Hamburg etc. Hamburg 1909.

73. - - Bemerkungen zur Gefäßpflanzenflora der Insel Föhr. Schriften Naturw. Ver. Schleswig-Holstein XV. Heft 1. 89/98 (1911).

74. - - : Bemerkungen zur Gefäßpflanzenflora der Inseln Sylt, Amrum und Helgoland. Schriften Naturw. Ver. Schleswig-Holstein XV. Heft 2. 307/320 (1912).

75. Kirmis, M.: Flora von Neumünster. Beilage zum Osterprogramm der Realschule zu Neumünster (1883).

76. Klatt, W.: Flora des Herzogtums Lauenburg. Hamburg 1865.

77. - - Nachtrag zur Flora des Herzogtums Lauenburg. Abhandlungen Botan. Ver. Provinz Brandenburg IX. 96/108 (1868).

7.. Kneucker, A.: Bemerkungen zu den Gramineae exsiccatae. Allg. Bot. Zeitschr. VIII. 28/33, 180/184 (1902), XII. 202/205 (1906).

79. Knuth, P.: Flora der Provinz Schleswig-Holstein usw. Leipzig 1887.

80. - - Schulflora der Provinz Schleswig-Holstein. Leipzig 1887.

81. - - Botanische Wanderungen auf der Insel Sylt. Tondern und Westerland 1890.

8:. - — Flora der nordfriesischen Inseln. Kiel 1895.

83. - - : Hlora von Helgoland. Kiel 1896.

84. - - - Die Pflanzenwelt der nordfriesischen Inseln. Schriften Naturw. Ver. Schleswig'Holstein IX. 1. 71/109 (1891).

55. - - - Grundzüge einer Entwicklungsgeschichte der. Pflanzenwelt in SchleswigHolstein. Schriften Naturw. Ver. Schleswig-Holstein VIII. Heft 1. 55/108 (1889). 
si. Knuth, P.: Bemerkungen zu meiner Flora der norlfriesischen Inseln mol meiner Flora von Helgoland. Allg. Bot. Zeitschr. IV. 107/110, 137/139 (1898).

47. Koch, W. D. J.: Synopsis Elorae Germanicae et Helveticne. Elit. II. 2 (1844).

88. - - : Synopsis der deutschen und Schweizer Flora. Frankfurt a. M. 1838.

\$!!. Körnicke, F., und Werner, H.: Handbuch des Getreidebaues. Band II. Bonn 1885.

90. Krause, E. H. L.: Beitrag zur Geschichte der Wiesenflora in Norddentschland. Engrer Bot. Jahrbücher XV. 387/400 (1893).

11. - - - : Mecklenburgische Nlora. Rostock 1893.

32. - - : Ubersicht der Flora von Holstein. Forschungsherichte der Biologischen Station zu Plön. T'eil 2, 20/30. Berlin 1894.

93. - - : Floristische Notizen. II. Grïser. Botanisches Zentralblatt IXXIII. $337 / 343,379 / 386$ (1898).

94. - _ - : Ein Besserungsversuch am System der Gramineen. Beihefte Botanisches Zentralb]att XXV. 421/489 (1910).

9.5. Kuphaldt, H. H.: Flora von Plön. Programm der Plöner Gelehrtenschule 1863.

96. Kuntz: Úber den Formenkreis der Calamagrostis lanceolata Roth. Beihefte Botanisches Zentralblatt XXIV. 2. $421 / 426$ (1909), XXVI. 2. 226/236 (1910).

97. Laban, F. C.: Flora von Hamburg. 3. Aufl. (1877), 4. Aufl. (1887).

98. - - - Flora des Herzogtums Holstein, des Fürstentums Lübeck, der Starlt Lübeck und deren Umgegend. Hamburg 1866.

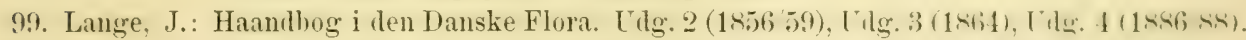

100. - - : Orersigt over de i Aarene 1865/66, 1867/68, 1869/71 i Danmark fundene sjeldene eller for den danske Flora nye Arter. Botanisk Tidsskir. 1. Reihe II. 30/67 (1867), 1. Reihe III. 67/125 (1869), 2. Reihe I. 244/322 (1872).

101. - - Pugillus plantarum imprimis hispanicarum. Naturhist. Forening Videnskab. Meddelelser 1860.

102. - - Rettelser og Tilföjelser til Haandbog i den Danske Flora. Kopenhagen 1897.

103. - - Flora Danica. Band XV. Heft 45 (1861); Band XVI. Heft 47 (1869), Heft 48 (1871); Band XVII. Heft 49 (1877), Heft 50 (1880), Heft 51 (1883).

104. - - und Steenstrup, J.: Flora Danica. Bd. XV. Heft 44 (1858).

105. Langmann, J. Fr.: Flora der Großherzogtümer Mecklenburg und . . . von Lauenburg, Lübeck .... 1. Aufl. Neustrelitz 1841, 3. Aufl. Schwerin 1871.

106. Leers, J. D.: Flora Herbormensis. Berlin 1789.

107. Lenz, H.: Übersicht der Lübeckischen Flora. Archiv des Vereins der Freunde der Naturgeschichte in Mecklenburg XXII. 35/88 (1869).

108. Liebmann, F. M.: Flora Danica. Band XTV. Heft 41 (1845), Heft 42 (1849); Band XV. Heft 43 (1852).

109. Lienau, W.: Die phanerogamischen Pffanzen des Fürstenthums Lübeck und seiner Umgebung. Eutin 1863.

110. Lohauss, K.: Der anatomische Bau der Lauhblätter der Festueaceen und dessen Bedlentumen für die Systematik. Bibliotheca Botanica Heft 63. Stuttgart 1905.

111. Marschall von Bieberstein, F.: Flora Taurico-Caucasica I. Charkow 1808.

112. Mauch, J. W.: Einige Notizen über Pflanzen, und Pflanzen kundige Mäner, in den Herzogthümern Schleswig, Holstein und Lauenburg. Neues staatsbürgerliches Magazin X. 509/540 (1841).

113. Meyer, G. F. W.: Chloris Hannoverana. Göttingen 1836.

114. - - : Flora Hamnoverana excursoria. Göttingen 1849.

115. Mößler, J. C.: Handbuch der Gewächskunde. 1. Aufl. Altona 1815.

116. Müller, F. v.: Breviarium plantarum Ducatus Slesvicensis austro-occidentalis. Flora $\mathbb{L}$. $473 / 480$ (18533). 
117. Müller, 0. F.: Flora Danica. Band IV. Heft 11 (1775), Heft 12 (1777); Band V. Heft 13 (1778), Heft 14 (1780), Heft 15 (1782).

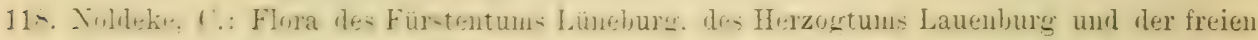
Stadt Hamburg. Celle 1890.

113. Nolte, E. F.: Noritiae Florae Holsaticae. Kiel 1826.

1ำ. - - : Handexemplar der "Noritiae Fl. Hols." mit handschriftlichen Bemerkungen.

121. Deder, G. C.: Flora Danica. Band I. Heft 3 (1764); Band II. Heft 4 (1765), Heft 5 (1766); Band III. Heft 7 (1768), Heft 8 (1769), Heft 9 (1770).

1:.2. Ohl, E.: Seltenere, charakteristische und verwilderte Pflanzen der Ungegend Kiels. Kiel 1889.

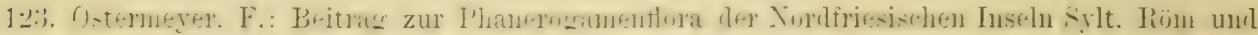
Fölır. Schriften Naturw. Ver. Schleswig-Holstein XIII. 1. 20/38 (1905).

121. Persoon, C. H.: Synopsis Plantarum I. Paris 1805.

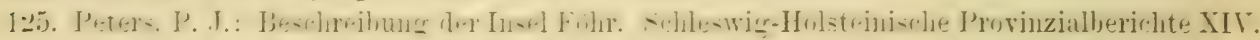
Heft 3. $440 / 449$ (1825).

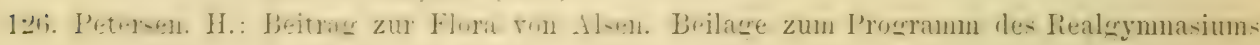
in Sonderburg 1891.

1\%خ. Petit, E.: Udkast til en floristisk Beskrivelse af Als. Botanisk Tidsskr. 13/41 (1880).

12-. Pieper, G. R.: Jahresberichte des Botanischen Vereins zu Hamburg: Nr. 7 Deutsche Bot. Monatsschr. XVI. 6 (1898), Nr. 8 D. B. MI. XVII 6/8 (1899), Nr. 9 D. B. M. XVIII. 5/6 (1900), Nr. 10 D. B. II. XLX. 8 (1901), Nr. 11 D. B. M. XX. 11/12 (1902), Nr. 12 D. B. M. XXI. 7/8 (1903), Nr. 13 Allg. Bot. Zeitschr. X. 12. XI. 1 (1904/05), Ir. 14 A. B. Z. XII. 2/5 (1906).

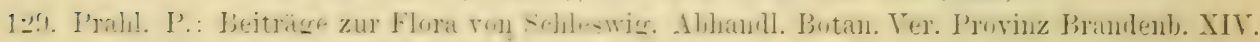
101/150 (1872), XVIII. 1/25 (1876).

1:31. - - Schulflora der Provinz Schleswig-Holstein usw. 1. Aufl. 1888, 2. Aufl. 1900, 4. Aufl. 1907. Kiel.

1:31. - - Kritische Flora der Provinz Schleswig-Holstein usw. Kiel 1890.

1:;2. - - Berichte der Deutschen Botanischen Gesellschaft: Schleswig-Holstein. IX. (125)/(128) (1891), X. (85)/(87) (1892).

1:3: - - Die Bastarde Calamagrostis Hartmaniana Fries und C. acutiflora (Schrader) DC: in Mecklenburg gefunden. Archiv Ver. Freunde d. Naturgesch. in Mecklenburg LIII. 170/176 (1899).

1:1. - - Mitteilungen zur Gattung Calamagrostis. Lübeck 1903.

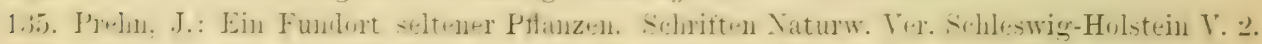
(1884).

1:3;. - - Einige Pflanzen Land Oldenburgs. Schriften Naturw. Ver. Schleswig-Holstein VI. 2. $57 / 64(1886)$.

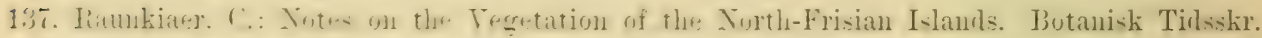
XVII. $179 / 196(1890)$.

15, Reichenbach, H. G.: Ernst Ferdinand Nolte, ein Hamburger Botaniker. In: Verzeichnis der Vorlesungen, welche am Hamburgischen Akademischen und Realgymnasium ron Ostern 1881 bis Ostem 1882 gehalten werden sollen. Hamburg 1881.

1:;.. Reichenbach, L.: Flora Germanica excursoria. Leipzig 1830.

1f1. - - Agrostographia Germanica. Leipzig 1834.

111. - - Icones Florae Germanicae et Helveticae. Teil I. Agrostographia Germanica. Leipzig 1850.

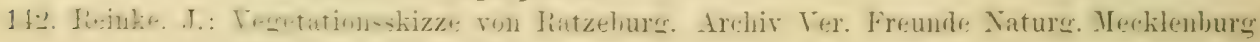
AXII. 88/105 (1869). 
143. Reinke, J.: Die Entwicklungsgeschichte der Dünen an der Westküste von Schleswig. Sitzungsberichte der Königlich Preubischen Akademie der Wissenschaften XIII. 1/16 (1903).

14. Ritter, Ch. WV.: Versuch einer Beschreibung der in den Herzogtümern Schleswig und Holstein usw. wildwachsenden P'flanzen mit sichtbarer Blüte. 'Tondern 1816. 115. - - Nachtrag zum "Versuch einer Beschreibumg usw.". Augustenburg 1817. 116. Roeper, J.: 'Zur Flora Mecklenburgs. 1. Teil. Programm Rostock 1843.

147. Rohweder, C., und Kähler, N.: Verzeichnis der Gefäßpflanzen der Umgegend von Neustart. Schriften Naturw.Ver.Schlesw.-HoIstein II.1.61/82 (1885)).

14.. Sander, J.: Botanische Ergebnisse aus dem Dänenkriegre. Abhandl. Bot. Ver. Provinz Brandenburg VI. 216/232 (1864).

149. Sanio, C.: Zahlenverhältnisse der Flora Preubens II. Abhamdl. Bot. Ver. Provinz Brandenburg XXXII, 5̄5/128 (1890).

150. Schiötz, Th.: Beretning om en botanisk Reise, foretaget i Sommeren 1858 i Landskabet mellem Slesvig, Rendsborg og Ekernförle sant paa Vesterhavs Öerne Amrom, För og Sild. Vilenskabelige Meddelelser fra den naturhistoriske Forening i Kjöbenhavin for Aaret 1860, 117/168 (1861).

1.j. Schmidt, J.: Beitrag zu einem Standortsverzeichnis der Phanerogamen iles südöstlichen Holstein. Schriften Naturw. Ver. Schleswig-Holstein. IIr. 35/101 (1878).

15\%. - . - : Die eingeschleppten und verwilderten Pflanzen der Hamburger Flora. Programm der Unterrichtsanstalten des Klosters St. Johannis zu Hamburg. 1890.

15:3. - - Neues aus der Flora Holsteins. Schriften Naturw. Ver. Schleswig-Holstein XI. 1. $87 / 98$ (1898).

151. - - Zur Flora von Röm. Deutsche Bot. Monatsschr. XVII. 7/10, 25/29 (1899). 15\%. - - : Jahresberichte des Botanischen Vereins zu Hamburg. Nr. 1. "Die Heimat" II. 7. 8 (1892), Nr. 2. a. a. 0. III. 7. 8 (1893), Nr. 3. a. a. 0. IV. 7. 8 (1894), Nr. 4. a. a. O. V. 6 (1895), Nr. 5. a. a. O. VI. 6 (1896), Nr. 15. Allg. Bot. Zeitschr. XII. 1. 2. (1906), Nr. 16/17. a. a. 0. XIV. 9. 10 (1908), Nr. 18. a. a. 0. XV. 11. 12. XVI. 1. $2(1909 / 10)$, Nr. 19. a. a. 0. XVII. 9. 10. 11 (1910), Ir. 20. a. a. O. XVIII. 7/9. 11. 12 (1912), XIX. 1\%2. 3 4. 5 (1913).

156. Schröder, J. v.: Geschichte und Beschreibung der Stadt Schleswig. Schleswig 1827.

157. - - - und Biernatzki, H.: 'Topographie der Herzogtümer Holstein und Lauenburg, des Fürstentums Lübeck und des Gebiets der freien und Hansestärlte Hamburg und Lübeck. Oldenburg i. H. 1855.

158. Schur, J. F.: Euumeratio plantarum Transsilvaniae. Wien 1866.

159. Seemen, 0. V.: Einige Mitteilungen über die in dem Herbar K. F. W. Jessen enthaltenen Pflanzen von der nordfriesischen Insel Amrum. Englers Jahrbücher XXVII. Beiheft 62.6/7 (1899).

160. Seckt: Sylt. Naturwissenschaftliche Wochenschrift. Neue Folge I. 73/77 (1901).

161. Sickman, J. R.: Enumeratio stirpium phanerogamicarum circa Hamburgum sponte crescentium. Hamburg 1836.

162. Smith, J. E.: Flora Brittamica I. London 1800.

163. Sonder, W.: Flora Hamburgensis. Hamburg 1851.

164. - - Flora. In: Festschrift zur 50. Versammlung deutscher Naturforscher und Ärzte 19/34 (1876).

165. Stoltenberg, N.: Beitrag zur Kemtnis der Flora Tonderns. Tondern 1877.

166. Thellung, A.: La Flore Adventice de Montpellier. Cherbourg 1912.

167. Timm, C. 'T.: Kritische und ergänzende Benserkungen, die Hamburger Flora betreffend. Verhandl. Naturw. Ver. Hamburg. Neue Folge IV. $38 / 92$ (1880), V. 82/85 (1881). 
168. Timm, (․ T.: Schleswig-Holstein. In: Berichte der Deutschen Botanischen Gesellschaft IV. CLXVI/CLXIX (1886), V. CII/CIV (1887).

169. - - und Prahl, P.: Schleswig-Holstein. In: Berichte der Deutschen Botanischen Gesellschaft VI. CXXII/CXXIV (1888), VII. (92)/(95) (1889), VIII. (123)/(126) (1890).

170. Torges, E.: Zur Gattung C'alcmagrostis. Mitteilungen des Thüringischen Botanischen Vereins. Neue Folge VII. 18/23 (1895), VIII. 13/16 (1896), XI. 78/93 (1899), XVII. 93/99 (1905).

171. Vahl, M.: Flora Danica. Band VI. Heft 16 (1787), Heft 17 (1790), Heft 18 (1792); Band VII. Heft 19 (1794), Heft 20 (1797), Heft 21 (1799).

172. Visiani, R. de: Flora Dalmatica I. Leipzig 1842.

173. Warming, E.: Fra Vesterharkystens Marskenge. Videnskabelige Meddelelser fra den naturhistoriske Forening i Kjöbenham for Aaret 1890. 206/239 (1891).

174. Weber, G. H.: Primitiae Florae Holsaticae. Kiel 1780.

175. - _ : Supplementum Primitiae Florae Holsaticae. Kiel 1787.

176. Weber, C.: Cher die Zusammensetzung des natürlichen Graslandes in Westholstein, Dithmarschen und Eiderstedt. Schriften Naturw. Ver. Schleswig-Holstein IX. 2 $179 / 217$ (1892).

177. Wibel, A. W. E. C.: Primitiae Florae Werthemensis. Jena 1797.

17s. Wildeman, E., und Durand, Th.: Prodrome de la Flore Belge. Bd. III. Brüssel 1899.

179. Willkomm, M., und Lange, J.: Prodromus Florae Hispanicae. Bd. I. Stuttgart 1861.

18(). Wirtgen, R.: Flora der preußischen Rheinprovinz. Bomn 1857.

181. Zarle: Die Zwischenformen vom Flughafer (Avena fatua) und Kulturhafer (Avena sativa) Frühlings Landwirtschaftliche Zeitung. Jahrgang 61. Heft 11. 369/384 (1912).

182. Zeemam, M.: Die systematische Bedeutung des Blattbaues der mitteleuropäischen AiraArten. Österreichische Botanische Zeitung LVI. 429/436, 457/461 (1906), LVII. 1/3 (1907). 


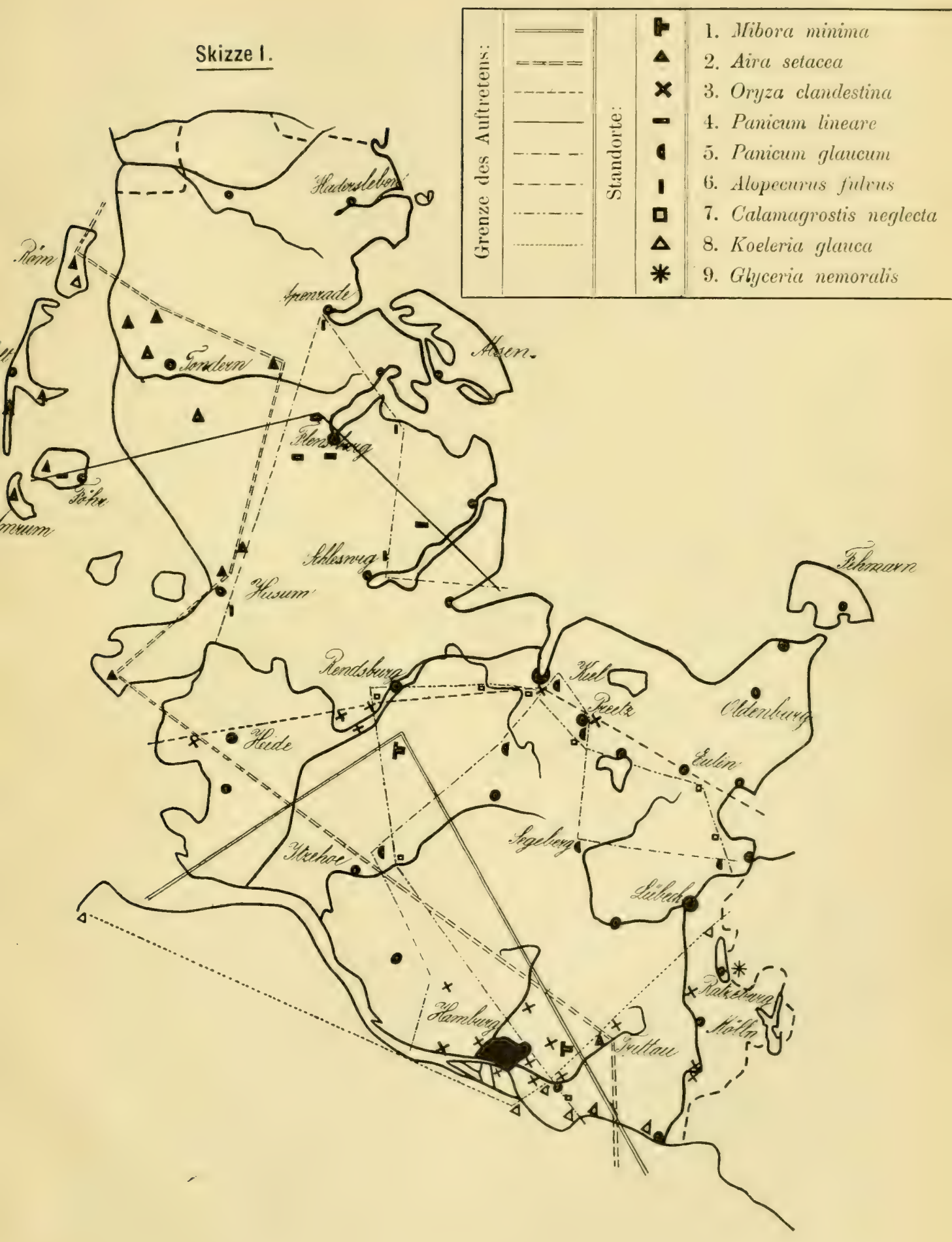



Skizze 2.

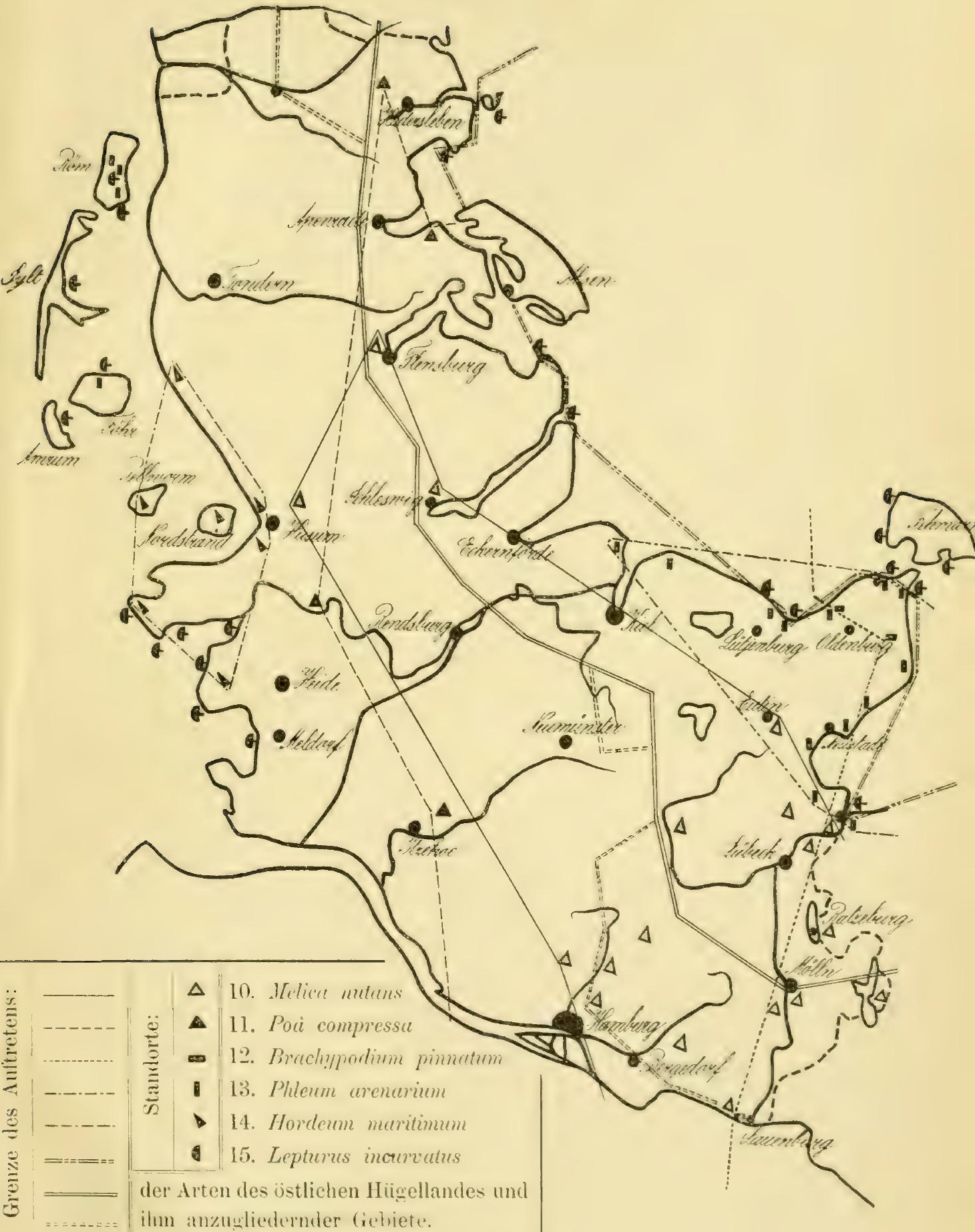





$\angle \mathrm{iE}$

\section{LIBRARY OF THE
MORTICULTURAL SDCIETY OF N.Y.}

598 MADISON AVENUE.

NEW YORK 
